\title{
DIMORPHISM AND INTEGRATION IN THE PELVES OF ANTHROPOID PRIMATES
}

A Dissertation
Presented to
The Faculty of the Graduate School
at the University of Missouri
In Partial Fulfillment
Of the Requirements for the Degree
Doctor of Philosophy

By

ELIZABETH A. MOFFETT

Dr. Carol V. Ward, Dissertation Supervisor

December, 2016 
(C) Copyright by Elizabeth A. Moffett 2016

All Rights Reserved 
The undersigned, appointed by the dean of the Graduate School, have examined the dissertation entitled

\title{
DIMORPHISM AND INTEGRATION IN THE PELVES OF ANTHROPOID PRIMATES
}

\author{
presented by Elizabeth Moffett, \\ a candidate for the degree of doctor of philosophy \\ and hereby certify that, in their opinion, it is worthy of acceptance.
}

Carol V. Ward

Kristina Aldridge

Gregory E. Blomquist

Kevin M. Middleton

J. Michael Plavcan 
This work is dedicated to Lloyd and Victoria Moffett, for their unceasing support 


\section{ACKNOWLEDGEMENTS}

First and foremost I would like to thank Carol Ward for being an outstanding mentor and advisor. Carol's guidance has been instrumental in shaping my research and career, and this dissertation certainly would not have been possible without her encouragement and feedback. Carol's enthusiasm for paleoanthropology is unrivaled, and if my career is half as prolific as hers, I will consider myself a success.

I also thank my committee - Kristina Aldridge, Greg Blomquist, Kevin Middleton, and Mike Plavcan - for helping me to develop and clearly communicate my research and ideas throughout graduate school, and especially with regards to my dissertation. I am fortunate to have had these amazing researchers helping me along the way. Kevin, Kris, and Mike - I am forever indebted to you for all of your assistance with statistical analyses. I also thank the other past and present Mizzou Integrative Anatomy faculty members and post docs for their support. Cheryl Hill, Scott Maddux, Rachel Dunn, and Mike Jorgensen provided the support that helped me see the light at the end of the tunnel. Graduate school is not easy, but your enthusiasm and helpfulness eased the path. I would also like to thank Bob Tague (Louisiana State University) for piquing my interest in pelvic dimorphism and shaping many of my ideas and research questions.

I could not have asked for a better group of people to attend graduate school with than the students of Mizzou IA. Ian Cost, Ian George, Ashley Hammond, Sharon 
Kuo, Faye McGechie, Sarah Peacock, Kaleb Sellers, Amanda Smolisnsky, and Henry Tsai - I will never forget the late nights we've spent drinking, painting, and watching awful movies. I am also grateful to graduate students of the Anthropology department, especially Colleen Young and Rachel Munds, for your friendship.

This past year, I have had great support at Stony Brook University. Fred Grine, Gabrielle Russo, Amy Lu, Brigitte Demes, Randy Sussman, and John Fleagle have all been incredibly welcoming toward me. I am forever indebted to your kindness.

My research would not have been possible without the kindness and generosity of museum curators. I thank Judy Chupasko, Neil Duncan, Lyman Jellema, Terry Kensler, Inbal Livne, Darrin Lunde, Aja Marcato, Bill Stanley, and Eileen Westwig for access to their collections. I am also thankful to Tim Cole for access to beta versions of his WinEDMA and EDMAProj 1.0.1 software, and to Joan Rischstmeier and Kris Aldridge for access to ScanDatabase. I also thank Kevin Middleton for his help with statistical procedures in $\mathrm{R}$.

For financial support, I am deeply grateful to the MU Life Sciences Fellowship Program, the Wenner-Gren and Louis B. Leakey Foundations, and the John Bies Foundation. Debbie Allen and Mark Hannink in MU Life Sciences were very supportive of me and my research. I also thank the administrators and staff of my granting agencies, Leslie Aiello (Wenner-Gren Foundation) and Paddy Moore (LSB Leakey Foundation). Thank you for giving me this amazing opportunity.

Last, I thank my friends and family for their understanding for these past six years. I have missed too many holidays, birthdays, weddings, births, funerals, and 
family gatherings in the pursuit of my degree, and despite my absences they have been unwavering in their support. I thank my grandfather, Curtis Cox, for being my inspiration to become an anthropologist, and for encouraging me along the way. My parents, Lloyd and Victoria Moffett, have provided me with emotional support, and I appreciate all of the sacrifices they have made over the years that have enabled me to pursue my dreams.

Ian George helped get me through many tough times over the past few years. Without him, it is unlikely that I would have been able to juggle all of the demands of graduate school. Thank you for keeping me sane and focused on my goals. 


\section{TABLE OF CONTENTS}

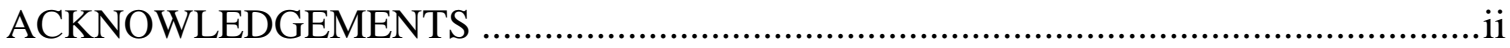

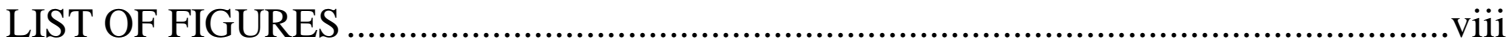

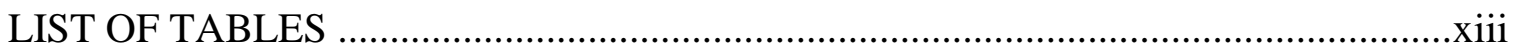

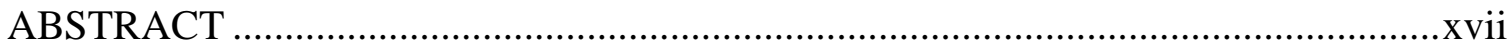

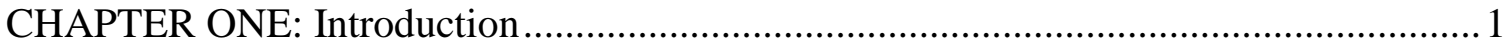

Cephalopelvic proportions and birth in anthropoids ....................................... 1

Factors hypothesized to influence pelvic dimorphism ......................................

Modularity and integration in the primate pelvis .............................................. 14

CHAPTER TWO: Dimorphism in the size and shape of the birth canals of anthropoid

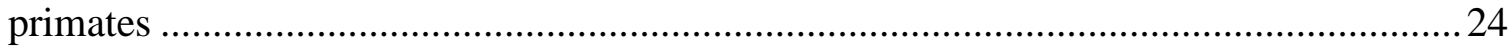

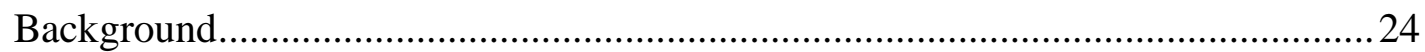

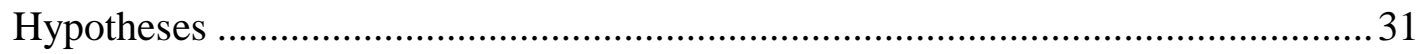

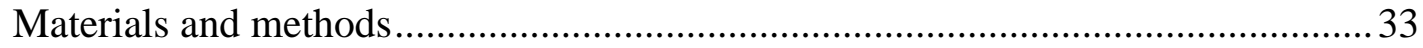

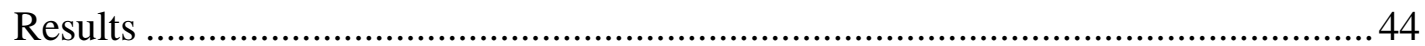

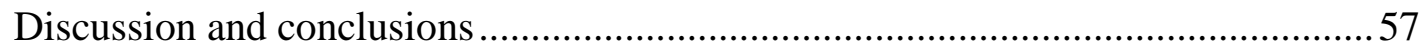


CHAPTER THREE: Dimorphism in relative sacral breadth among Catarrhine

primates

92

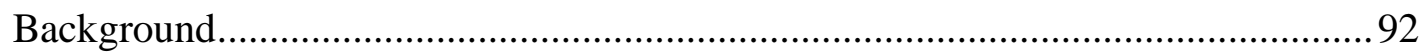

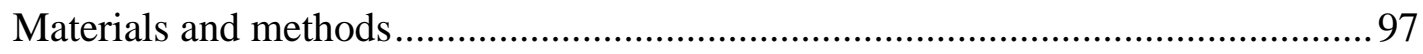

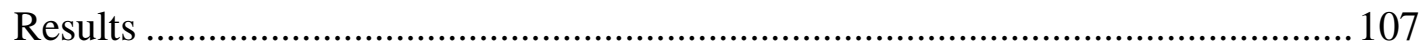

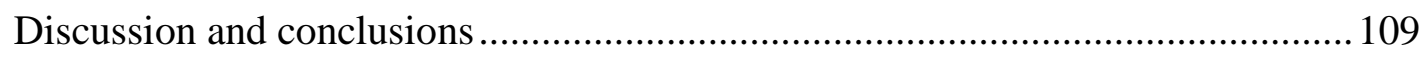

CHAPTER FOUR: Dimorphism in non-obstetric pelvic morphology in anthropoid primates

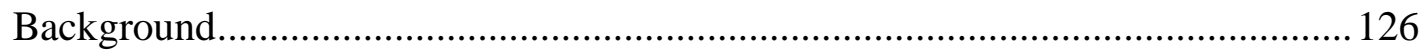

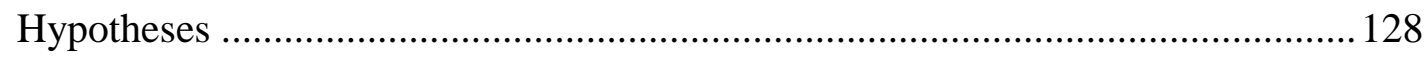

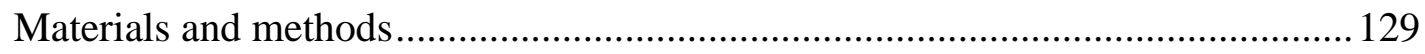

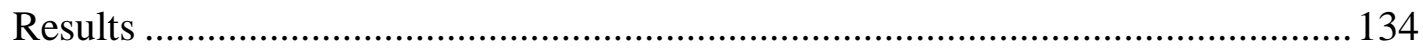

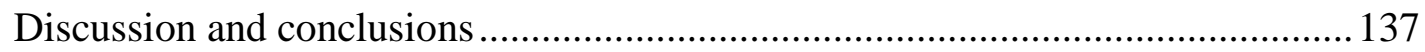

CHAPTER FIVE: Integration in the anthropoid birth canal and non-obstetric pelvis... 157

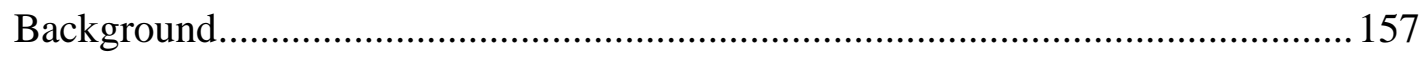

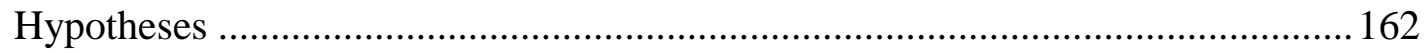

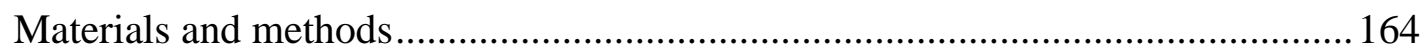


Results

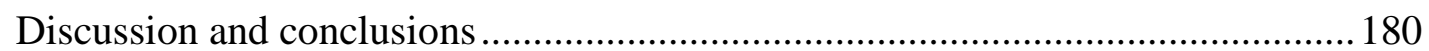

CHAPTER SIX: Summary and Future Directions ..................................................... 195

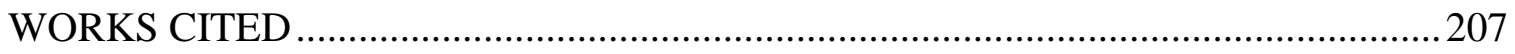

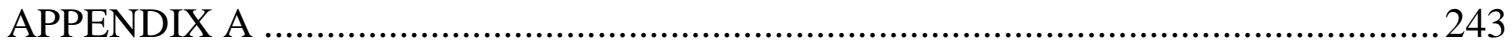

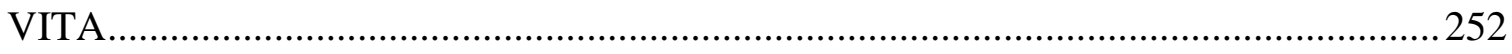




\section{LIST OF FIGURES}

Figure 1.1: Cephalopelvic proportions in primates

Figure 1.2: Superior view of a fetal skull showing relative anteroposterior and mediolateral dimensions .

Figure 1.3: Relationship between maternal and neonatal body size in anthropoid primates

Figure 1.4: Allometry of primate brain development

Figure 1.5: The three planes of the birth canal - inlet, midplane, and outlet 22

Figure 1.6: Fetal head aligning with the pelvic inlet.....

Figure 1.7: Fetal rotation in humans versus non-human primates

Figure 2.1: Landmarks collected on the birth canal 63

Figure 2.2: Linear distances of the birth canal

Figure 2.3: Birth canal shape overlap between sexes

Figure 2.4a: Principal components analysis of birth canal shape in Homo sapiens

Figure 2.4b: Euclidean distance matrix analysis of birth canal shape in Homo sapiens.. 83

Figure 2.5a: Principal components analysis of birth canal shape in Hylobates lar..... .84 
Figure 2.5b: Euclidean distance matrix analysis of birth canal shape in Hylobates lar... 84

Figure 2.6a: Principal components analysis of birth canal shape in Macaca mulatta .....85

Figure 2.6b: Euclidean distance matrix analysis of birth canal shape in Macaca mulatta 85

Figure 2.7a: Principal components analysis of birth canal shape in Nasalis larvatus .....86

Figure 2.7b: Euclidean distance matrix analysis of birth canal shape in Nasalis larvatus 86

Figure 2.8a: Principal components analysis of birth canal shape in Papio anubis

Figure 2.8b: Euclidean distance matrix analysis of birth canal shape in Papio anubis ...87

Figure 2.9a: Principal components analysis of birth canal shape in Saimiri sciureus .....88

Figure 2.9b: Euclidean distance matrix analysis of birth canal shape in Saimiri sciureus 88

Figure 2.10a: Principal components analysis of birth canal shape in Gorilla gorilla ......89

Figure 2.10b: Euclidean distance matrix analysis of birth canal shape in Gorilla gorilla 89

Figure 2.11a: Principal components analysis of birth canal shape in Pan troglodytes ....90

Figure 2.11b: Euclidean distance matrix analysis of birth canal shape in Pan

troglodytes. .90

Figure 2.12a: Principal components analysis of birth canal shape in Alouatta seniculus .91

Figure 2.12b: Euclidean distance matrix analysis of birth canal shape in Alouatta seniculus 91 
Figure 3.1: Scatter plots of (A) body mass dimorphism index versus lumbar vertebral body dimorphism index and (B) body mass dimorphism index versus cephalopelvic proportions.

Figure 3.2: Measurements of the (A) sacrum and (B) lumbar vertebra 120

Figure 3.3: Anterior views of male and female sacra of: (a) Hylobates lar; (b) Nasalis larvatus; (c) Homo sapiens; (d) Gorilla gorilla

Figure 4.1: Landmarks of the non-obstetric pelvis

Figure 4.2: Overlap between the sexes in non-obstetric pelvis shape 146

Figure 4.3a: Principal components analysis of non-obstetric pelvis shape in Homo sapiens . 148

Figure 4.3b: Euclidean distance matrix analysis of non-obstetric pelvis shape in Homo sapiens 148

Figure 4.4a: Principal components analysis of non-obstetric pelvis shape in Hylobates lar

Figure 4.4b: Euclidean distance matrix analysis of non-obstetric pelvis shape in Hylobates lar

Figure 4.5a: Principal components analysis of non-obstetric pelvis shape in Macaca mulatta. 
Figure 4.5b: Euclidean distance matrix analysis of non-obstetric pelvis shape in Macaca mulatta

Figure 4.6a: Principal components analysis of non-obstetric pelvis shape in Nasalis larvauts

Figure 4.6b: Euclidean distance matrix analysis of non-obstetric pelvis shape in Nasalis larvatus

Figure 4.7a: Principal components analysis of non-obstetric pelvis shape in Papio anubis

Figure 4.7b: Euclidean distance matrix analysis of non-obstetric pelvis shape in Papio anubis

Figure 4.8a: Principal components analysis of non-obstetric pelvis shape in Saimiri sciureus

Figure 4.8b: Euclidean distance matrix analysis of non-obstetric pelvis shape in Saimiri

sciureus

Figure 4.9a: Principal components analysis of non-obstetric pelvis shape in Gorilla gorilla

Figure 4.9b: Euclidean distance matrix analysis of non-obstetric pelvis shape in Gorilla gorilla 
Figure 4.10a: Principal components analysis of non-obstetric pelvis shape in Pan troglodytes

Figure 4.10b: Euclidean distance matrix analysis of non-obstetric pelvis shape in Pan troglodytes 155

Figure 4.11a: Principal components analysis of non-obstetric pelvis shape in Alouatta seniculus 156

Figure 4.11b: Euclidean distance matrix analysis of non-obstetric pelvis shape in Alouatta seniculus 156

Figure 5.1: Landmarks of the birth canal and non-obstetric pelvis 187

Figure 5.2: Linear distances of the birth canal 188

Figure 5.3: Linear distances of the non-obstetric pelvis 189 


\section{LIST OF TABLES}

Table 2.1: Numbers of individuals, body mass dimorphism, and cephalopelvic

proportions

Table 2.2: Birth canal landmarks 63

Table 2.3: Birth canal dimension calculations .64

Table 2.4: Reduced major axis regressions of natural logged birth canal size against S1

body breadth .65

Table 2.5: Reduced major axis regression of natural logged birth canal dimensions

against $\mathrm{S} 1$ body breadth

Table 2.6: Student's t-tests for dimorphism in absolute and relative birth canal size......66

Table 2.7: Student's t-tests for dimorphism in absolute and relative birth canal

dimensions

Table 2.8: Student's t-tests results for differences between males and females in residuals from reduced major axis regressions of pelvic size variables with S1 body

breadth .74

Table 2.9: Dimorphism index ranks

Table 2.10: Results for discriminant function analysis of the influence of sex on intraspecies principal components scores for birth canal shape .79 
Table 2.11: Student's t-tests for dimorphism in birth canal shape along each principal component 80

Table 2.12: Euclidean distance matrix analysis FORM test of male and female differences in overall birth canal shape within species 80

Table 2.13: Shape of the pelvic planes in female and male anthropoids

Table 2.14: Shape overlap between sexes

Table 3.1: Sampled taxa 118

Table 3.2: Isometry test results

Table 3.3: Hypotheses and predictions outlined in text

Table 3.4: Randomization tests (2-tailed) for sexual dimorphism in vertebral measurements

Table 3.5: Randomization test results for sexual dimorphism in relative sacral and relative lumbar breadths

Table 4.1: False pelvis landmarks

Table 4.2: Reduced major axis regressions of natural logged non-obstetric pelvis dimensions against logged S1body breadth 144

Table 4.3: Dimorphism in non-obstetric pelvic size: Student's t-tests on residuals 144

Table 4.4: Magnitude of dimorphism ranks for the non-obstetric pelvis 145 
Table 4.5: Shape overlap between sexes

Table 4.6: Student's t-tests for dimorphism in birth canal shape

Table 4.7: Euclidean distance matrix analyses of male and female differences in overall non-obstetric pelvs shape within species

Table 5.1: Hypotheses and predictions 186

Table 5.2: Landmarks of the birth canal.

Table 5.3: Landmarks of the non-obstetric pelvis 187

Table 5.4: Modularity testing for birth canal and non-obstetric pelvis 190

Table 5.5: Magnitudes of integration in birth canal linear distances across anthropoids191

Table 5.6: Difference of means test of difference in integration magnitudes between taxa for birth canal distances

Table 5.7: Differences in the magnitudes of integration in birth canal linear distances between males and females across anthropoids

Table 5.8: Magnitudes of integration in non-obstetric pelvic linear distances

Table 5.9: Similarity in integration magnitudes between taxa for non-obstetric pelvic distances 192

Table 5.10: Differences in the magnitudes of integration in non-obstetric linear distances between males and females across anthropoids 192 
Table 5.11: Mantel tests of matrix similarity in interspecies integration patterns

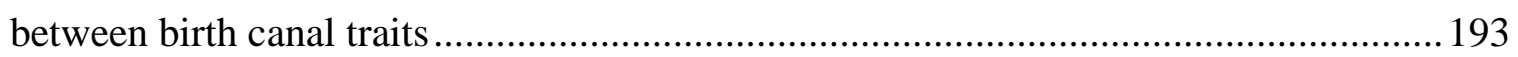

Table 5.12: Similarity in birth canal integration patterns between males and females.. 193

Table 5.13: Mantel tests of matrix similarity in integration patterns between non-

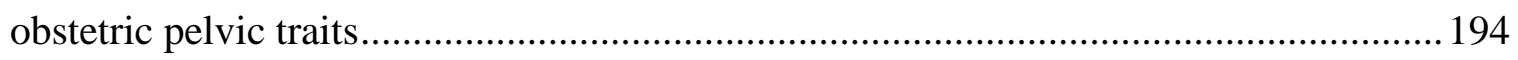

Table 5.14: Similarity in non-obstetric integration patterns between males and females 194 


\title{
DIMORPHISM AND INTEGRATION IN THE PELVES OF ANTHROPOID PRIMATES
}

Elizabeth A. Moffett

\author{
Dr. Carol V. Ward, Dissertation Supervisor
}

\begin{abstract}
The link between obstetric demand (natural selection for birth) and pelvic morphology in anthropoids has remained ambiguous for the past century. Though it is assumed that the dramatic sexual dimorphism seen in modern human pelves is due to obstetric demand, this does not explain why other primates that give birth to relatively small neonates also exhibit pelvic dimorphism. This thesis explores the correlation between obstetric demand and dimorphism and morphological integration in anthropoid pelves. Dimorphism in birth canal and non-obstetric pelvic morphology is present across most primates regardless of obstetric demand, but those primates that give birth to relatively large babies have the most pelvic sexual dimorphism. Sexual dimorphism in sacral breadth is likewise most apparent in species that give birth to large neonates relative to the maternal birth canal. In species that give birth to large neonates, females also have higher magnitudes of integration in birth canal morphology compared to males, but this is not true in species that give birth to small neonates. This study demonstrates a clear link between obstetric demand, pelvic dimorphism, and the magnitudes of pelvic integration in anthropoid primates.




\section{CHAPTER 1: INTRODUCTION}

Reproductive success is one of the most important requirements of any animal's biology - unsuccessful birth immediately and dramatically affects the fitness of both mother and offspring. Therefore, obstetric (birth-related) selection likely has considerable effects on pelvic morphology in species with a tight fit between the maternal pelvis and neonate. However, the extent to which obstetric selection has shaped the primate pelvis is poorly characterized - as important as birth is hypothesized to be in shaping pelvic morphology, we actually know relatively little about how birth affects primate pelvic morphology in anthropoid primates. How do the three dimensional shapes of the pelvis differ between males and females due to obstetric selection? What different patterns of pelvic sexual dimorphism evolved across anthropoid species in response to obstetric demand? Are primate pelvic integration patterns influenced by obstetric demand? These gaps in our knowledge limit the inferences we can make about the functional morphology of the pelvis, sexual dimorphism, and the evolution of cephalopelvic proportions in primates.

The purpose of this thesis is to address the effects of obstetric demand on sexual dimorphism and integration in the pelves of anthropoids. Chapter 2 quantifies the patterns of sexual dimorphism in the birth canals of anthropoid taxa and the effects of obstetric demand on the magnitudes of birth canal dimorphism seen in these taxa. Chapter 3 further assesses the effects of obstetric demand on sexual dimorphism in the 
sacrum of anthropoids. Then, Chapter 4 quantifies the extent of sexual dimorphism in parts of the pelvis not directly related to the birth canal (termed here the "non-obstetric pelvis") and how these relate to obstetric demand. Finally, Chapter 5 quantifies the effects of obstetric demand on the patterns and magnitudes of morphological integration (covariation between various individual parts) of anthropoid pelves.

\section{CEPHALOPELVIC PROPORTIONS AND BIRTH IN ANTHROPOIDS}

Cephalopelvic proportions - the ratio of neonatal head diameters to maternal birth canal dimensions (Schultz, 1949; Leutenegger, 1982; Rosenberg, 1992; Li, 2002; Rosenberg and Trevathan, 2002) - are the metric by which obstetric demand is frequently quantified in primates (Schultz, 1949; Leutenegger 1970, 1974; Lindburg, 1982; Stoller 1995). Large cephalopelvic proportions, or large neonatal head size compared to maternal pelvic diameters, are common among primates (Schultz, 1949; Leutenegger, 1970, 1974; Stoller, 1995), especially among monkeys. Among New World monkeys, Ateles and Saimiri are known to have large cephalopelvic proportions, while Alouatta has smaller proportions than Ateles or Saimiri (Schultz 1949; Leutenegger 1970, 1974; Stoller 1995). Among Old World monkeys, Papio, Nasalis, and Macaca have large cephalopelvic proportions (Schultz 1949; Leutenegger 1970, 1974; Stoller 1995); there are no known Old World monkeys with small proportions. In contrast, the living apes generally tend to have small cephalopelvic proportions (Figure 1.1); two notable exceptions include Hylobates and Homo (Schultz, 1949; Leutenegger, 1982). Birthing large-headed newborns has further been shown to lead to 
strenuous labors in nonhuman primates (Bowden et al., 1967; Hopf, 1967; Schultz, 1969; Brandt and Mitchell, 1971; Christen, 1974; Phillips, 1976). For example, squirrel monkeys (Saimiri sciureus) have a prolonged and difficult birth process (Bowden et al., 1967; Hopf, 1967), with dystocia, or a mismatch between neonatal head size and maternal birth canal size, occurring in roughly $25 \%$ of labors (Rasmussen et al., 1980). In contrast, large-bodied apes such as Pongo and Gorilla have small cephalopelvic proportions (Schultz, 1949; Leutenegger, 1982) and relatively easy labors compared to humans (Brandt and Mitchell, 1971).

Neonatal head size shape will obviously affect both neonatal head dimensions and cephalopelvic proportions. In all primates, the sagittal (anteroposterior) dimension of the neonatal cranium is larger than the transverse (mediolateral) dimension (Figure 1.2; Schultz, 1949; Leutenegger, 1974), and the widest transverse diameter of the neonatal cranium is near the occiput (Figure 1.2). Brain size and body size are negatively allometric across mammals (and primates), such that smaller animals have relatively larger brains (Jerison, 1973). There is also a negatively allometric relationship between neonatal body weight and maternal body weight (Figure 1.3; slope $\left.=0.69, r^{2}=0.98\right)$, such that the relative size of the neonate becomes disproportionately larger as maternal body size decreases (Leutenegger, 1982; Wells et al., 2012), although humans have a larger neonatal size than is predicted based upon maternal body size (Leutenegger, 1982; DeSilva and Lesnik, 2008). Moreover, neonatal brain mass scales negatively with adult brain mass in primates (Martin, 1983; DeSilva and Lesnik, 2008; DeSilva, 2011), meaning that in large-bodied apes, more brain development occurs postnatally compared to smaller-bodied monkeys (Figure 
1.4). These allometric relationships may account for the small cephalopelvic proportions found in non-human large-bodied apes.

The shape and dimensions of the maternal bony birth canal will also affect cephalopelvic proportions. There are three areas of constriction in the birth canal traditionally defined for humans and other primates (Figure 1.5): the pelvic inlet, midplane, and outlet (see also Rosenberg, 1992). These "planes" (though not actually planar in all primates) define areas of the pelvis that are common sites of cephalopelvic disproportion, or a mismatch between the neonatal head and birth canal (Leutenegger, 1974; Lindburg, 1982; Trevathan, 1987; Tague, 1991; Rosenberg, 1992; Rosenberg and Trevathan, 2002). The inlet is bounded anteriorly by the superior pubic symphysis and pectineal line, laterally by the linea arcuata, and posteriorly by the superior sacral alae and anterior first sacral vertebral body. The midplane is bounded anteriorly by the inferior pubic symphysis, laterally by the ischial spines, and posteriorly by the midsacrum. The outlet is bounded anteriorly by the inferior pubic symphysis, laterally by the postero-medial ischial tuberosities, and posteriorly by the inferior sacrum. In all primates besides hominins, the dorsoventral dimensions of all three planes exceed the transverse dimensions (Schultz, 1949; Leutenegger, 1974). In monkeys, the broadest part of the birth canal is in the dorsal portion of the birth canal (creating a dorsally capacious but ventrally compressed birth canal), whereas humans also have ventrally widened birth canals such that the entire birth canal is rounded (Rosenberg and Trevathan, 2002).

How the neonatal head engages the birth canal during birth also influences cephalopelvic proportions. Generally, the fetus will orient itself so that the maximum 
diameter of the cranium (dorsoventral) is aligned with the maximum diameter of the maternal pelvic planes (Joulin, 1864). Accordingly, in non-human primates, the neonate modally enters the birth canal facing ventrally (relative to the mother), such that the longest cranial dimension (dorsoventral) is aligned with the largest diameter of the birth canal (also dorsoventral) and the broadest part of the cranium (near the occiput) is near the dorsal portion of the birth canal (Figure 1.6). The neonate remains in this position to emerge face-forward (Figure 1.7; Brandt and Mitchell, 1971; Lindburg, 1982; Rosenberg, 1992; Rosenberg and Trevathan, 1996; Trevathan, 1996; Rosenberg and Trevathan, 2002). In contrast, humans have birth canals in which the maximum diameter of the inlet (the transverse diameter) is rotated 90 degrees (Figure 1.7) compared to those of the midplane and outlet (the dorsoventral diameters) (Schultz, 1949; Lindburg, 1982; Tague and Lovejoy, 1986; Trevathan, 1987; Rosenberg, 1992; Ruff, 1995; Rosenberg and Trevathan, 1996; Trevathan, 1996; Rosenberg and Trevathan, 2002; Walrath, 2003; Franciscus, 2009; Brown, 2011). Therefore, in humans the head engages the inlet facing laterally (i.e., with the dorsoventral cranial dimension aligned with the transverse pelvic inlet diameter; Figure 1.6), but when the head reaches the midplane, it rotates (Figure 1.7) so that the dorsoventral cranial dimension engages the dorsoventral diameter of the midplane (Lindburg, 1982; Tague and Lovejoy, 1986; Trevathan, 1987; Rosenberg, 1992; Rosenberg and Trevathan, 1996; Trevathan, 1996; Rosenberg and Trevathan, 2002). Then, because humans have a ventrally expanded midplane and outlet compared to other primates, the broadest portion of the neonate cranium (near the occiput) aligns with the ventral part of the midplane and outlet, and the neonate emerges from the birth 
canal facing dorsally (Brandt and Mitchell, 1971; Lindburg, 1982; Trevathan, 1987;

Rosenberg and Trevathan, 1996; Trevathan, 1996; Walrath, 1997; Rosenberg and Trevathan, 2002; Franciscus, 2009).

Cephalopelvic proportions in primates have previously been calculated between the neonatal head and maternal pelvic inlet using what are assumed to be the most common birth orientations (Figure 1.6; Schultz, 1949; Leutenegger, 1970, 1974; Stoller, 1995). Any alternative alignments of neonates in the birth canal would change how the neonatal cranium engages the birth canal. In fact, it has been observed that many human neonates do not rotate in the traditionally described manner (Caldwell and Moloy, 1933; Caldwell et al., 1934; Calkins, 1939; Steele and Javert, 1942; Walrath, 2003), and fetal rotation sometimes occurs in both Saimiri and Papio such that neonates engage the inlet in an oblique orientation (Stoller, 1995). Nonetheless, because current models of cephalopelvic proportions assume that the dorsoventral diameter of the fetal head is oriented so that it aligns with the maximum diameters of the birth canal, these models provide conservative estimates of cephalopelvic proportions in primates.

One limiting factor of using cephalopelvic proportions to quantify obstetric demand is that diameters of the birth canal and neonatal head may not be static during birth. Birth canal size may be able to increase during birth due to ligamentous deformation - the result of the hormone relaxin (Thorp and Fray, 1938; Hartman and Straus, 1939; Weiss et al., 1981; Fuchs and Fuchs, 1984; Weiss, 1984; Germain and Ferre, 1987; Sherwood, 1988; Maclennan, 1991; Goldsmith et al., 1995). Further, in humans the neonatal head can deform during birth due to the presence of cranial 
fontanelles in human neonates (Aisenson, 1950; Acheson and Jefferson, 1954; Popich and Smith, 1972; Davies et al., 1975; Faix, 1982; Duc and Largo, 1986; Malas and Sulak, 2000; Faro et al., 2005; Paladini et al., 2008; Shajari et al., 2011). However, it is unknown how consistent ligamentous and neonatal cranial deformation are within primate species, and whether or not these phenomena are pervasive across taxa. Future studies should aim to quantify ligament and neonatal head deformation and their effects on cephalopelvic proportions.

Another factor influencing obstetric selection is the relative size and rigidity of the shoulders of the neonate (Trevathan and Rosenberg, 2000). Shoulder dystocia, or mismatch between the size of the shoulders and the birth canal, is known to occur in humans (Benedetti and Gabbe, 1978; Acker et al., 1985; Gross et al., 1987; Nocon et al., 1993). However, rates of shoulder dystocia among non-human primates are not well-studied, and how the shoulders are able to deform in the birth canal during birth across primates is unexplored. Because much more is known about cephalopelvic proportions in anthropoids (Schultz, 1949; Leutenegger, 1970, 1974; Stoller, 1995), cephalopelvic proportions are a more frequently-used metric to evaluate obstetric difficulty in primates.

\section{FACTORS HYPOTHESIZED TO INFLUENCE PELVIC DIMORPHISM}

\section{Birth and the "obstetric dilemma"}

Sexual dimorphism is a difference in morphology between males and females within a single species (Darwin, 1896). Dimorphism can arise by sexual selection (i.e., 
one sex selecting for a specific morphology in the opposite sex) or by differing selective pressures on sexes imposed by their environments or biological requirements (Darwin, 1896; Rensch, 1960; Lande, 1980b; Hedrick and Temeles, 1989; Shine, 1989; Plavcan, 2001; Fairbairn and Blanckenhorn, 2007; Plavcan, 2011). The magnitude of dimorphism in any structure can be thought of as a result of selection on both male and female traits (Plavcan, 2011).

In Homo, both males and females are argued to be under selection for reduced distances between the acetabulae and between the acetabular joints and sacrum (Washburn, 1950, 1960; Pinkerton, 1973; Zihlman, 1978) because this decreases the required hip abductor forces during bipedal locomotion (Ruff, 1995). However, females are also hypothesized to experience birth-related selection to maintain an obstetrically sufficient bony pelvis for passage of a relatively large-headed neonate (Washburn, 1960). This conflict between selection on the pelvis for narrower dimensions to facilitate bipedalism and selection for a larger birth canal to allow birth has been coined the "obstetric dilemma" (Washburn, 1960).

This "obstetric dilemma" in females has been hypothesized to be a primary factor driving dimorphic pelvic morphology in humans (Berge et al., 1984; Tague and Lovejoy, 1986; Abitbol, 1987b; Hager, 1989; Rosenberg, 1992; Abitbol, 1996; Rosenberg and Trevathan, 2002; but see Dunsworth et al., 2012; Wells et al., 2012). Indeed, human females have been found to exhibit relatively (controlling for body size) larger transverse and dorsoventral dimensions of the pelvic inlet (Thoms and Greulich, 1940; Young and Ince, 1940; Segebarth-Orban, 1980) and midplane (Holland et al., 1982; Abitbol, 1988, Hager, 1989; Tague, 1992; Correia, 2005; Kurki, 2007; Brown, 
2011), as well as for the transverse diameters of the pelvic outlet (Holland et al., 1982; Hager, 1989; Tague, 1992; Correia, 2005; Brown, 2011). Females also have relatively longer pubic bones (Washburn, 1948) and relatively wider sacra (Tague, 2007; Moffett et al., 2013). Additionally, humans are dimorphic in some aspects of the pelvis which are not directly part of the birth canal, including biacetabular (Tague, 1992) and biiliac breadth (Ruff and Hayes, 1983; Ruff, 1987, 1995; Lovejoy, 2005), and it is presumed that these dimorphisms are a consequence of dimorphism in the bony birth canal.

In addition to influencing modern human pelvic dimorphism, it has been argued that our species' strenuous obstetric demand was a primary factor influencing pelvic form during the Pleistocene (Ruff, 1995; Lovejoy, 2005). In fact, it is hypothesized that a tight fit between the neonatal head and maternal birth canal similar to modern humans could have evolved among Homo erectus (Walker and Ruff, 1993; Ruff, 1995; Simpson et al., 2008; but see DeSilva, 2011), and it almost certainly present among early H. sapiens and Neandertals (Arsuaga et al., 1999; Rosenberg et al., 2006; Ponce de León et al., 2008; Franciscus, 2009; Weaver and Hublin, 2009).

Our species' difficult birth has also been related to the evolution of other unique human traits and social behaviors including obligate birth assistance from other humans, increased infant dependency after birth, and increased cooperative behaviors during and after pregnancy (Trevathan, 1996; Rosenberg and Trevathan, 2002). Humans also give birth to young with a relatively lower percentage of prenatal brain growth completed before birth compared to other primates (Krogman, 1951; Washburn, 1960; Lovejoy, 1981; Rosenberg, 1992). Thus, this “obstetric dilemma" has 
historically been thought to be a key feature of our lineage, impacting our morphology, developmental trajectories, and sociality.

However, recent research questions whether the "obstetric dilemma" is actually a contributing factor to pelvic dimorphism and secondary altriciality in our species (Dunsworth et al., 2012; Wells et.al, 2012). Instead, it has been argued that our secondary altriciality is due to the metabolic constraints of gestating a large fetus (Dunsworth et al., 2012), and that the extremely tight fit between the birth canal and neonatal head in modern humans is a result of a shift to agricultural subsistence (Wells et al., 2012), and populations dependent on foraging and/or hunting (the context in which modern humans first evolved) should not exhibit such an extreme obstetric dilemma. Further, it has recently been argued that the overall wider pelvis of females does not negatively impact locomotor efficiency (Warrener et al., 2015). Last, it has been questioned whether the "obstetric dilemma" is even a uniquely human feature (Wells et al., 2012), as females exhibit relatively larger birth canals than males in many mammalian species (Gardner, 1936; Schultz, 1949; Dunmire, 1955; Crelin and Newton, 1969; Leutenegger, 1970, 1973; Chapman et al., 1994; Tague, 2003; Berdnikovs et al., 2007; Schutz et al., 2009a; Schutz et al., 2009b).

Yet, regardless of whether the "obstetric dilemma" is uniquely human or even whether it exists at all, the highly dimorphic morphology of the pelvis in our species is typically attributed to selection for birth (Turner, 1885; Paterson, 1893; Derry, 1912; De Souza, 1913; Emmons, 1913; Trotter, 1926; Caldwell and Moloy, 1933; Howells and Hotelling, 1936; Thoms, 1937; Greulich, 1938; Thoms and Greulich, 1940; Young and Ince, 1940; Letterman, 1941; Nicholson and Allen, 1945; Heyns, 1947; Flander, 
1978; Flander and Corruccini, 1980; Segebarth-Orban, 1980; Abitbol, 1988; Hager, 1989; Milne, 1990; Tague, 1992; Arsuaga and Carretero, 1994; LaVelle, 1995; Abitbol, 1996; Hager, 1996; Walrath, 1997; Tague, 2000; Walrath, 2003; Correia et al., 2005; Kurki, 2007; Novak, 2007; Tague, 2007; Benazzi et al., 2009; Kurki, 2011; Novak et al., 2012; Moffett et al., 2013).

The presence of a range of cephalopelvic proportions across anthropoid primates (Figure 1.1) provides an excellent opportunity to test the hypothesis that large cephalopelvic proportions influence dimorphism in the pelvis. Yet, previous research has not resolved whether large cephalopelvic are the primary cause of dimorphism in the birth canal across anthropoid primates (Schultz, 1949; Leutenegger, 1970; Mobb and Wood, 1977; Steudel, 1981b; Wood and Chamberlain, 1986; Li, 2002; Tague, 2005). Therefore, it is critical to quantify the patterns of dimorphism in the birth canal across anthropoids as well as quantify the role of obstetric selection in shaping pelvic dimorphism both within and across anthropoids.

\section{Body size and body size dimorphism}

Some previous research has implicated allometric scaling in influencing pelvic dimorphism across primates (Schultz, 1949; Steudel, 1981b, 1984; Tague, 2005). Ischial length, pubic length, lower iliac height, and sagittal inlet diameter scale with negative allometry across primates (Steudel, 1984; but see Lewton, 2015 for ischial length). Accordingly, there is an overall evolutionary trend within primates for ischial length, pubic length, lower iliac height, and sagittal inlet diameter, to become relatively 
smaller as animals become larger. In species in which males are much larger than females for body size, it is plausible that allometric scaling may account for sexual dimorphism in these pelvic morphologies (Steudel, 1981b).

\section{Sexual differences in ecology or locomotor repertoire}

The pelvis serves as an attachment site for the hindlimbs as well as for muscles related to locomotion. Muscles which govern hindlimb and trunk movement attach to the pelvis. To the pubis, adductors attach along the caudal portion, while abdominal muscles attach to the superior portion. The quadratus lumborum, abdominal muscles and latissimus dorsi muscles also attach to the superior portion of the ilium, whereas iliacus, lateral hip rotators, and hip abductors attach to the posterior and lateral portions of the ilium in hominids. Hip flexors attach at the anterior portion of the ilium, and hip extensors attach to the posterior and caudal portions of the ischium. The pelvic floor muscles attach to the caudal and posterior ischium as well as the caudal pubis. The stresses and strains associated with locomotion will affect pelvic form (Badoux, 1974; Dalstra and Huiskes, 1995; Lewton, 2010), and pelvic morphology is thus hypothesized to be related to habitual or obligate locomotor repertoires assumed by primates (Waterman, 1929; Steudel, 1981; Ward, 1991, 1993; MacLatchy, 1998; Lewton, 2010).

Primates exhibit a range of positional and locomotor behaviors, including pronogrady and orthogrady, suspension, clinging and leaping, bipedalism, and terrestrial and arboreal quadrupedalism. Humans, who are bipeds, have a 
craniocaudally compressed pelvis, reduced inferior iliac height, and laterally flaring ilia compared to other primates (Johanson et al., 1982; Tague and Lovejoy, 1986; Lovejoy et al., 1999; Lovejoy, 2005), as well as enlarged and medially protruding ischial spines (Abitbol 1988; Lovejoy 2005) and widened sacra (Robinson, 1972; Leutenegger and Kelly, 1977; Abitbol, 1987a; Lovejoy, 2005) compared to other primates. Primates which use suspensory locomotion have wide and coronally-oriented iliac blades (Waterman, 1929; Schultz, 1930; Reynolds, 1931; Reynolds and Hooton, 1936; Schultz, 1936, 1961; Benton, 1967; Ward, 1991; Lycett and von CramonTaubadel, 2013; Lewton, 2015; Morgan et al., 2015), small iliac tuberosities (Waterman, 1929; Ward, 1991), greater lumbar entrapment and cranio-caudally expanded pelvescompared to quadrupeds. In contrast, quadrupedal primates tend to have more sagittally-oriented iliac blades, a narrower posterior ilium, and large ischial tuberosities (Waterman, 1929; Schultz, 1930; Reynolds, 1931; Schultz, 1936, 1961; Benton, 1967; Ward, 1991) compared to bipedal and suspensory primates.

In primates, especially in species that have high levels of body size dimorphism like gorillas, males and females may use different frequencies of various locomotor behaviors (Remis, 1995). Thus, disparities in male and female pelvic morphology may be due to differences in biomechanics and/or slight locomotor differences between males and females of disparate body sizes within a single species (Steudel, 1981b; 1984). Pelvic dimorphism, although usually thought to result from selection on the pelves of females, may also be the result of selection on males, especially within species which are largely divergent in body size and may exhibit slight differences in 
locomotor behaviors due to such large body size dimorphism (Moffett et al., 2013), such as gorillas.

\section{MODULARITY AND INTEGRATION IN THE PRIMATE PELVIS}

\section{Defining modularity and integration}

Modularity and integration describe processes by which evolution shapes biological complexity and imposes constraints upon the forms of organisms. Morphological integration (Olson and Miller, 1951; Olson and Miller, 1958), or covariation between phenotypic traits, is the result of either selection for covariation of these phenotypes and/or a pleiotropic (i.e., one gene affecting multiple traits) linkage of these phenotypes (Lande, 1979, 1980a). Selection for covariation may alter the magnitude of covariation among traits (i.e, increase covariation of morphological traits), or alter a pattern of morphological integration (i.e., alter which traits covary) (Cheverud, 1996; Ackermann and Cheverud, 2000; Marroig and Cheverud, 2001).

As it is currently used, the term "modularity" describes a unit or number of units of a phenotype which: 1) collectively serve a primary functional role (Wagner, 1996); 2) are integrated morphologically, genetically, functionally, and/or developmentally (Cheverud, 1988, 1996; Wagner, 1996; Klingenberg, 2008); 3) are somewhat independent (uncorrelated) of other such units (Wagner, 1996; Klingenberg, 2008); and 4) exhibit hierarchical levels of interaction with other sets of traits (Wagner and Altenberg, 1996; Magwene, 2001). Thus, selection on one module can produce changes within the module that do not greatly affect other modules (Wagner, 1996; 
Wagner and Altenberg, 1996; Magwene, 2001; Wagner et al., 2007; Klingenberg, 2008; Klingenberg and Marugán-Lobón, 2013). For example, if we envision that the ilium is a separate module from the pubis, selection for increased iliac breadth should not produce a correlated change in the length of the pubis. However, selection for increased iliac breadth could produce a correlated change in iliac neck thickness because these two traits are within the same (hypothetical) module.

While any modular system must necessarily have integration between the traits within that module, integrated traits are not necessarily modular. To illustrate this point, imagine six separate linear distances. In the first instance, three of these traits tightly covary with one another, and the other three traits also tightly covary with one another. However, the three traits in the first group do not covary with the three traits in the second group. In this first instance, these sets of traits can be categorized as modular. Now envision that all six linear distances strongly covary with one another. In this case, these linear distances would be said to be integrated, but they are not arranged into modules.

\section{The effects of obstetric selection on pelvic integration in anthropoids}

It is possible that obstetric demand influences integration and modularity in the primate pelvis (Lewton, 2013). The primate pelvis has been characterized as being comprised of two modules, the ilium and the ischiopubis (Lewton, 2013). The ischiopubic region participates heavily in the birth canal, while the ilium does not (excepting the linea arcuata), so it is possible that these two modules are differentially 
affected by obstetric selection. Compared to other apes (which have smaller cephalopelvic proportions), humans have increased modularity (reduced covariation) between the linear distances within the birth canal and other pelvic traits (Grabowski, 2013). Increased modularity in the human pelvis compared to other apes could be the result of increased obstetric selection in humans (compared to other apes) (Grabowski et al., 2011; Grabowski, 2013). If this is the case, other primates with large cephalopelvic proportions should also exhibit reduced integration (and increased modularity) between the birth canal and non-obstetric pelvis. However, previous studies have not tested this hypothesis.

It should be expected that any birth-related selection should lead to increased integration (and less variation) within the birth canal among species with large cephalopelvic proportions, because traits within the birth canal should be under coselection. For example, if obstetric selection increases pubic length, sacral breadth, and inlet breadth among all species with large cephalopelvic proportions, these traits may be more highly integrated among species with large cephalopelvic proportions than in species with small cephalopelvic proportions. Evidence indicates that Hylobates and Homo have increased birth canal integration compared to other apes (Grabowski, 2013), and these two taxa also have large cephalopelvic proportions compared to other apes. This result could indicate that the selective pressure exerted by large cephalopelvic proportions results in higher correlations among obstetrically-relevant traits. This hypothesis should be further examined across anthropoids.

Because all primates are under selection for locomotion (regardless of cephalopelvic proportions) it might be expected that integration within the non- 
obstetric pelvis should either be unaffected or decreased by obstetric selection. For example, the covariation between ischium length, acetabular size, and iliac breadth should not be affected by relative neonate size, so integration levels between these distances should be similar across taxa regardless of obstetric demand. 


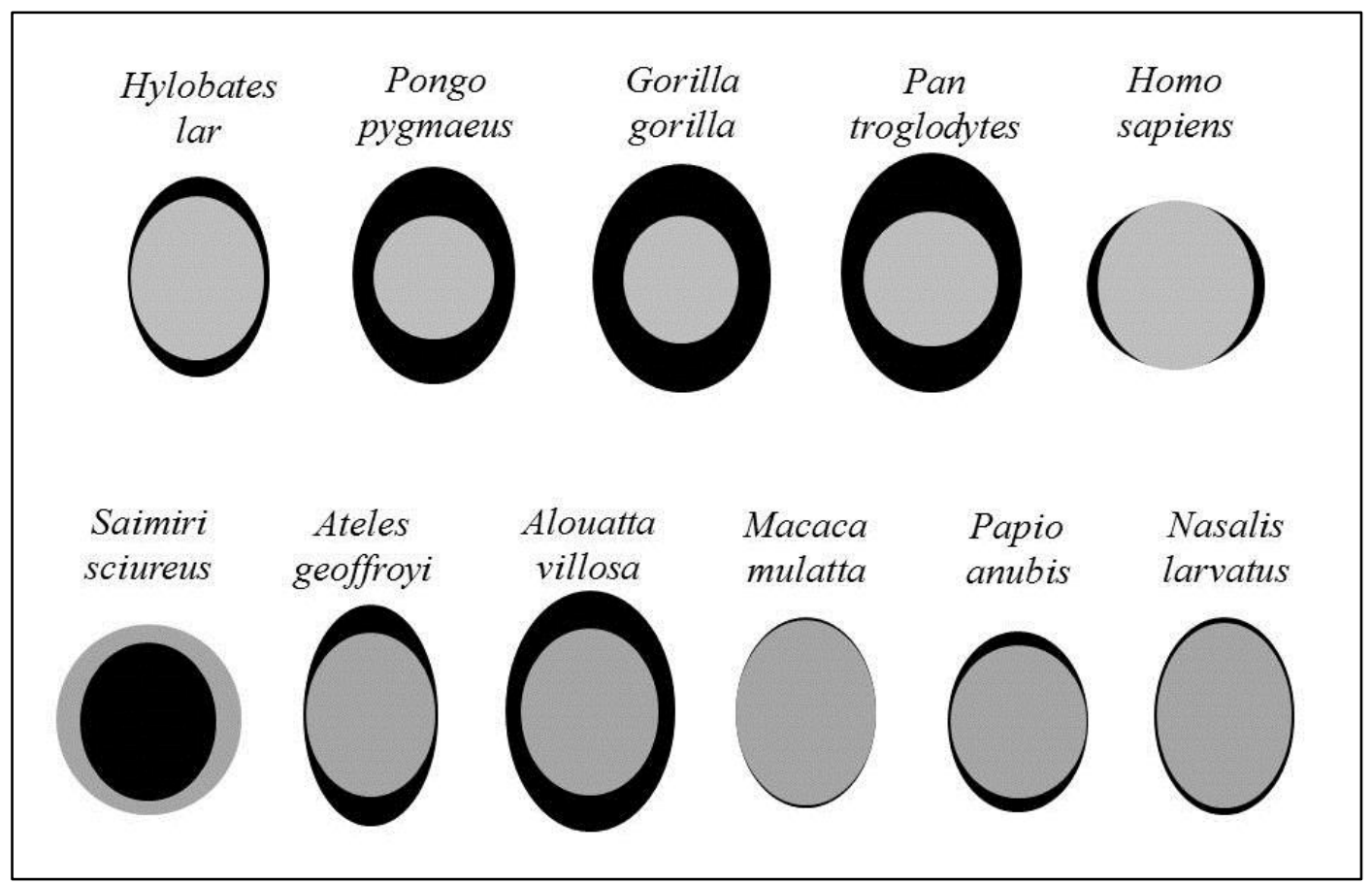

Figure 1.1: Cephalopelvic proportions in primates. Gray represents neonatal head diameters and black represents maternal pelvic inlet diameters at the time of birth. Figure modified from Schultz (1949); data for cephalopelvic proportions in Alouatta, Papio, Saimiri from Leutenegger (1982) and Stoller (1995). 


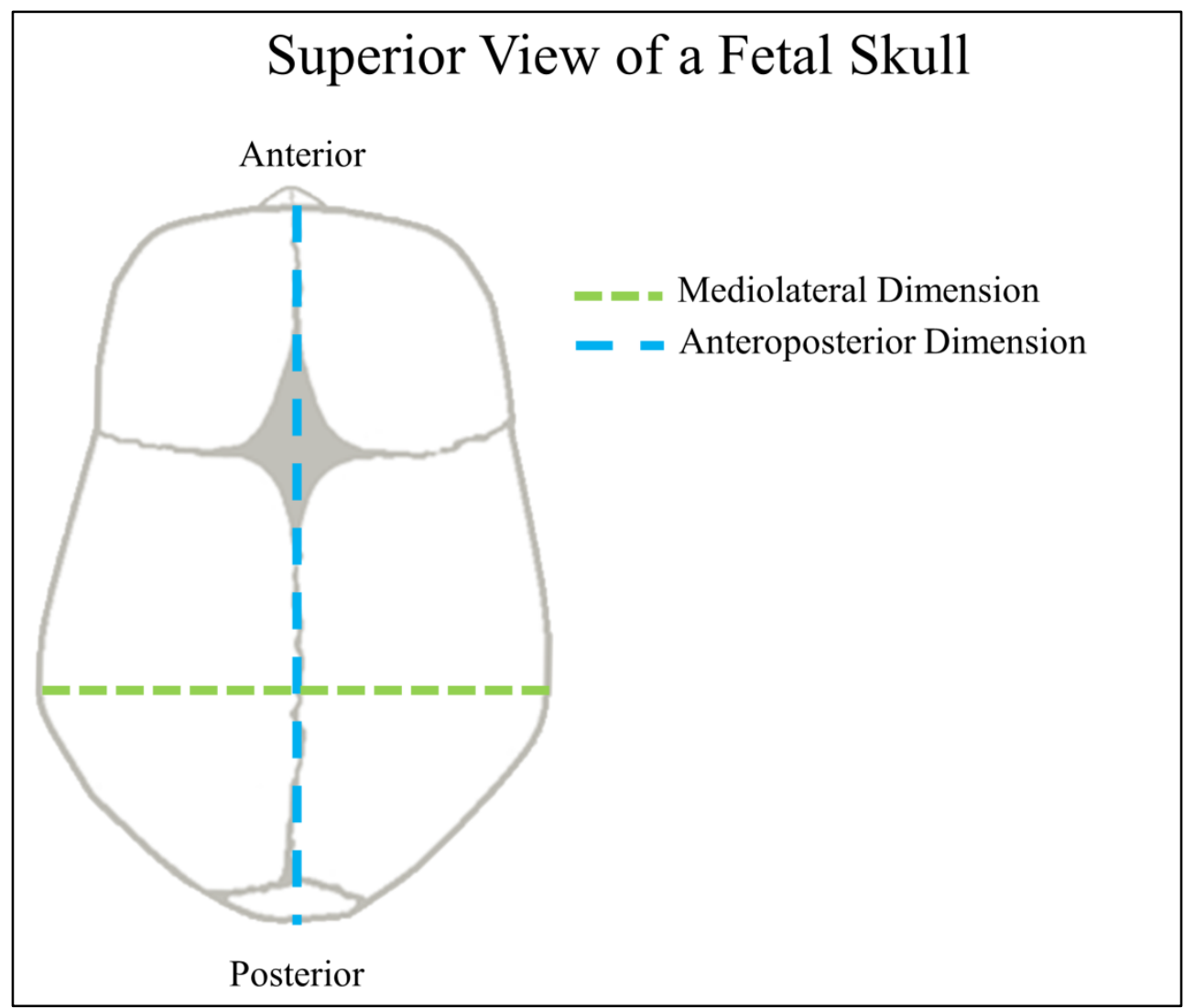

Figure 1.2: Superior view of a fetal skull showing the relative anteroposterior and mediolateral dimensions of the skull. Figure modified from Moffett and Aldridge (2013). 


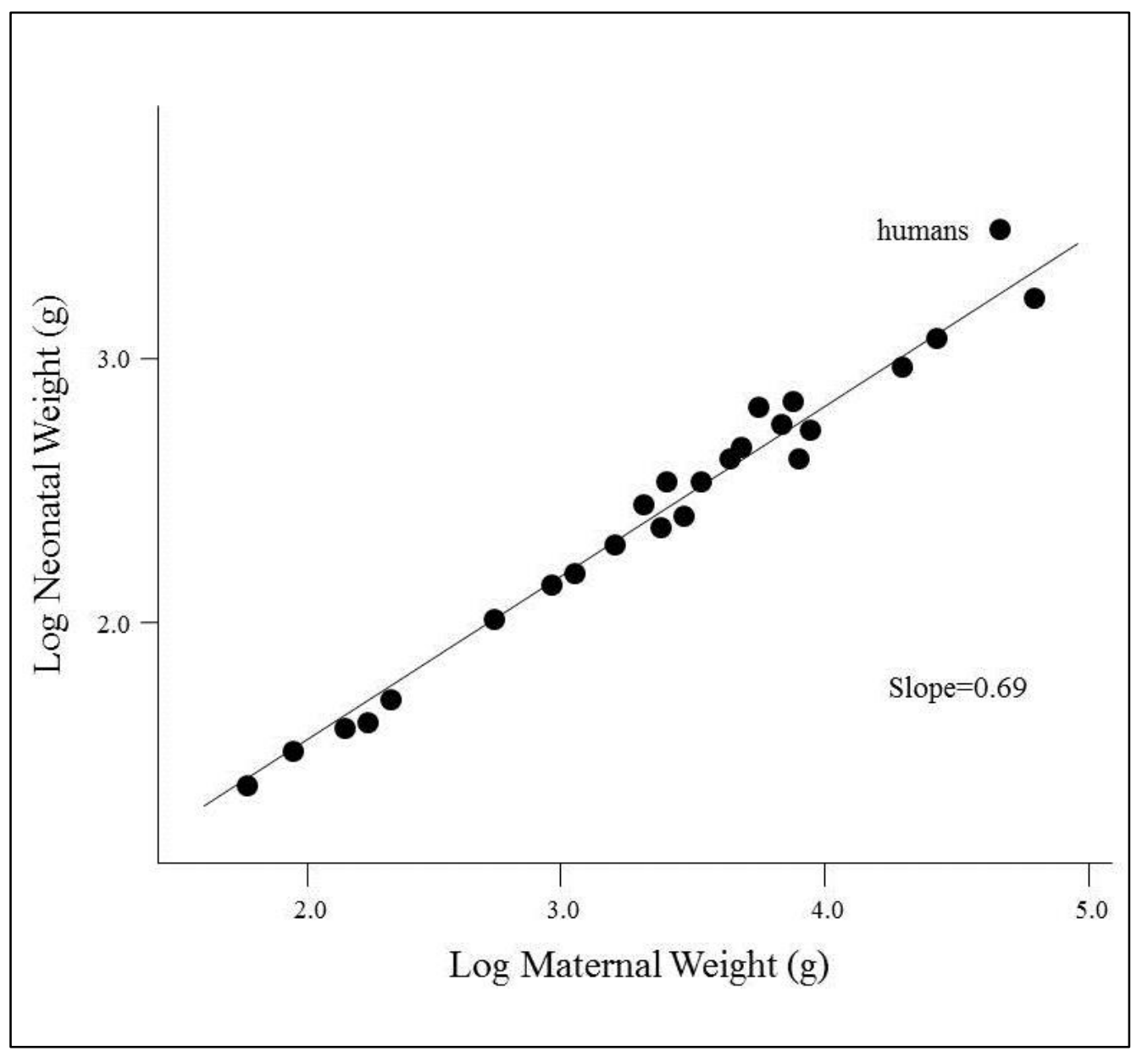

Figure 1.3: Relationship between maternal and neonatal body size in anthropoid primates. Figure adapted from Leutenegger (1982). 


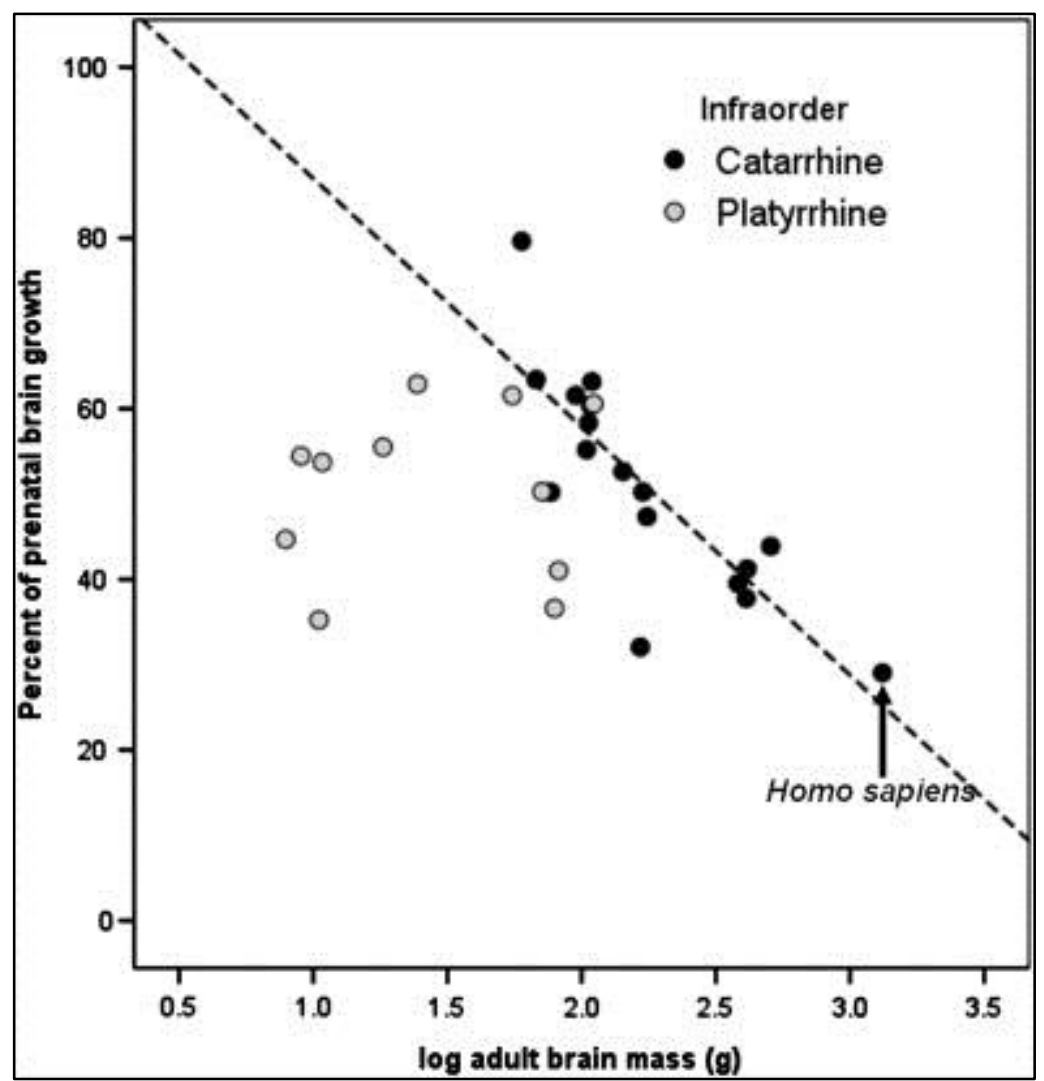

Figure 1.4: Allometry of primate brain development $\left(\mathrm{r}^{2}=0.66\right)$. Figure modified from DeSilva and Lesnik (2008). Note the negatively allometric relationship between prenantal brain growth and total adult brain mass among Catarrhines. 


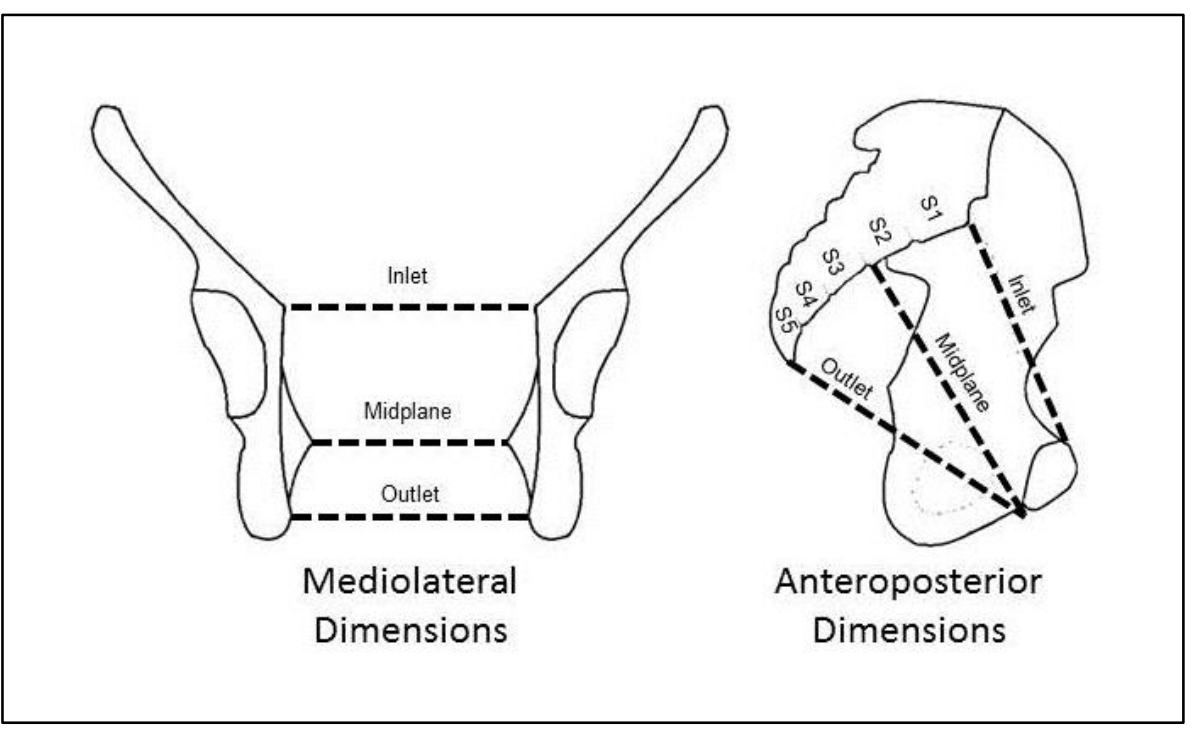

Figure 1.5: The three planes of the birth canal - inlet, midplane, and outlet. Figure modified from Rosenberg (1992).

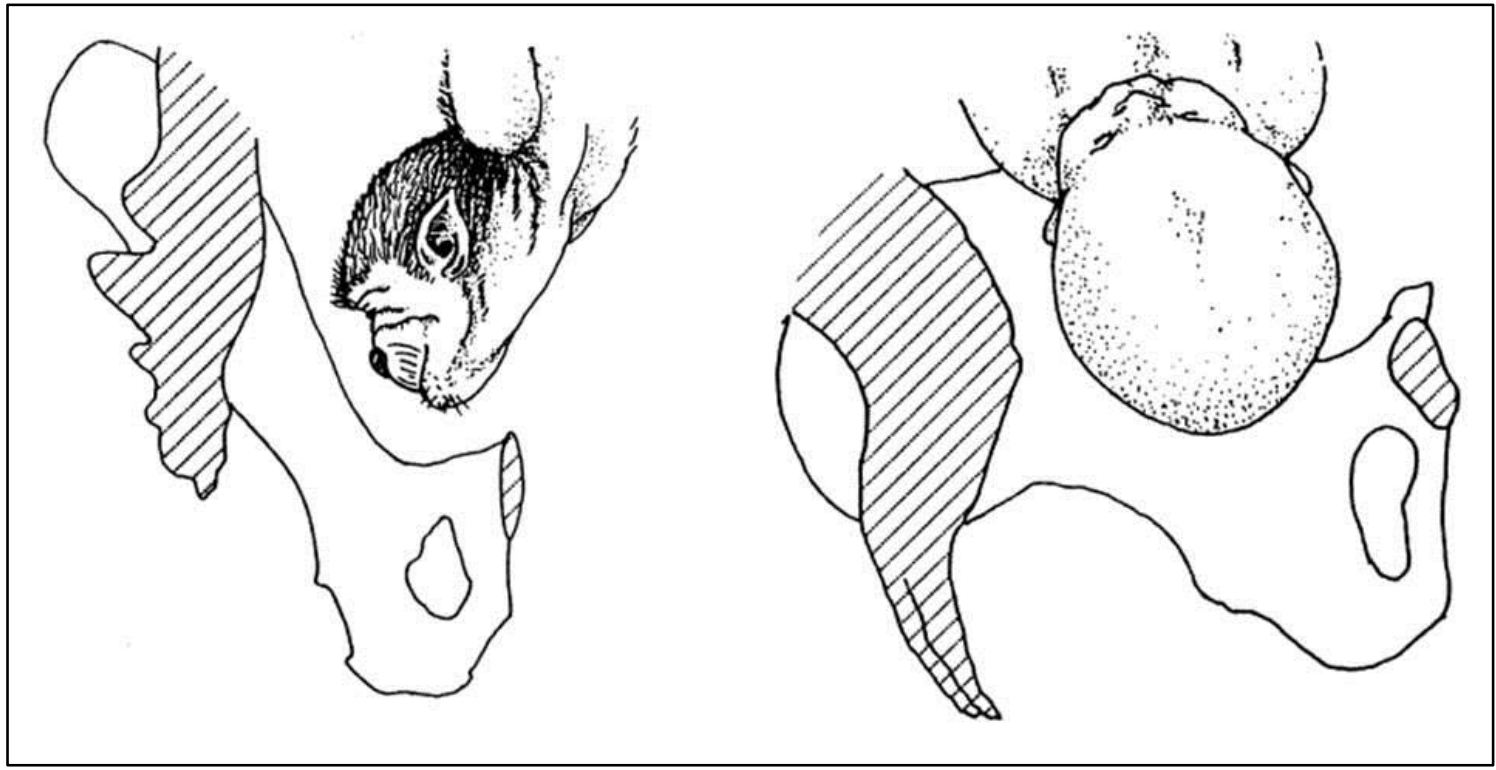

Figure 1.6: Fetal head aligning with the pelvic inlet in (a) non-human primates; and (b) humans. Figure modified from Trevathan (1987), original artwork by Aldine de Gruyter. 


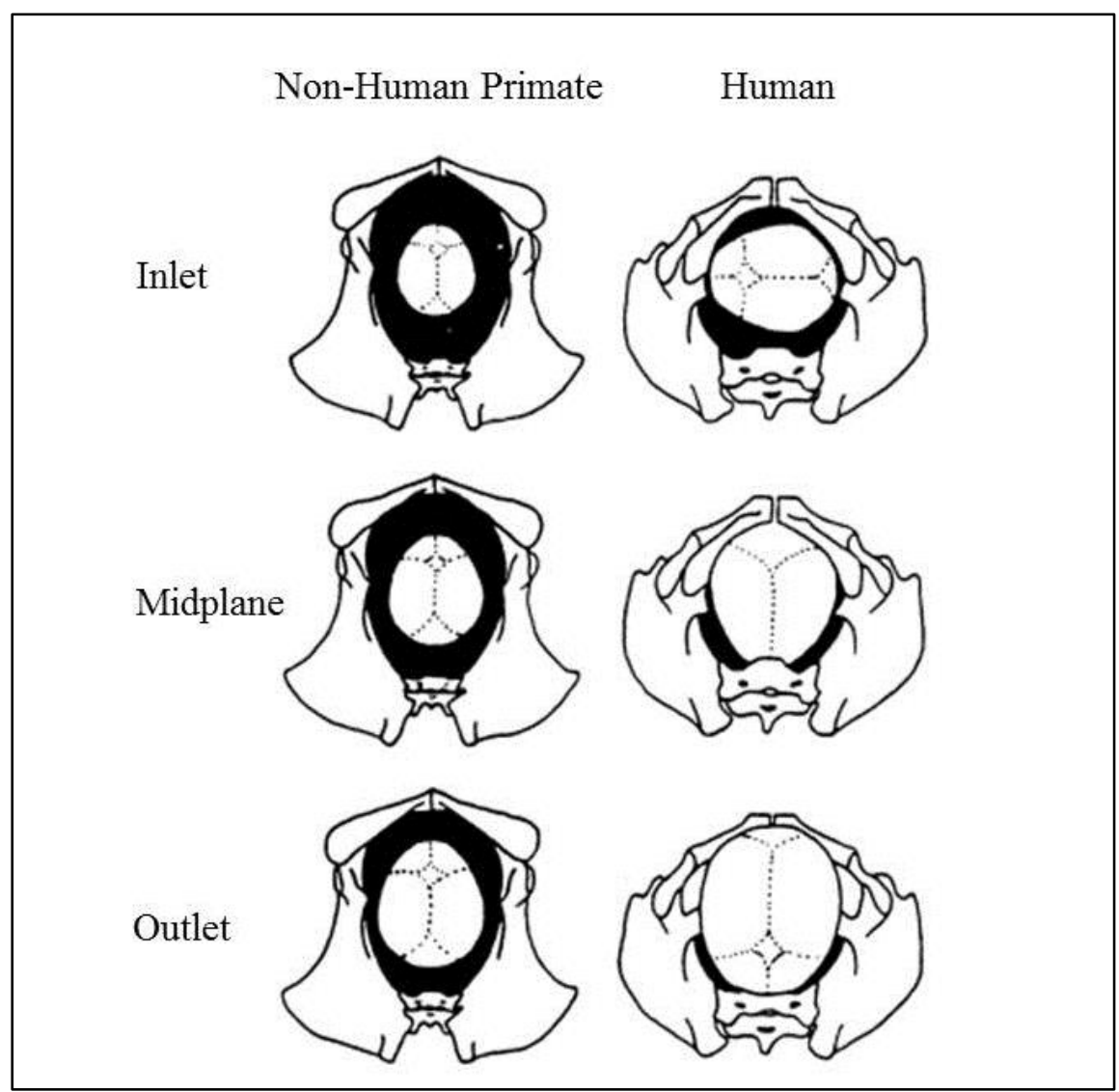

Figure 1.7: Fetal rotation in humans versus non-rotation in non-human primates. Figure modified from Lovejoy (2005). 


\section{CHAPTER 2: DIMORPHISM IN THE SIZE AND SHAPE OF THE BIRTH CANALS OF ANTHROPOID PRIMATES}

\section{BACKGROUND}

Human females give birth to large neonates relative to maternal body and birth canal size (Schultz, 1949; Leutenegger, 1974), and the hypothesis that the female pelvis is adapted to the selective pressures of giving birth to such large neonates appears wellsupported (Washburn, 1948; Tague, 1989, 1992; Lovejoy, 2005). Although females are smaller than males for both stature and body mass, females have a more spacious birth canal than males (Turner, 1885; Caldwell and Moloy, 1933; Greulich, 1938; Young and Ince, 1940; Coleman, 1969; Krogman and Işcan, 1986; Hager, 1989; Tague, 1989, 1992; Walrath, 1997; Correia et al., 2005; Kurki, 2007; Brown, 2011; Kurki, 2011). Across human populations, females have larger circumferences of the pelvic inlet, midplane, and outlet than males, both absolutely and relative to body size (Tague, 1992). Females also have mediolaterally wider midplanes (Holland et al., 1982; Hager, 1989; Tague, 1991; 1992; Correia, 2005; Brown, 2011) and outlets (Holland et al., 1982; Hager, 1989; Tague, 1992; Correia, 2005; Brown, 2011; but see Krogman and Işcan, 1986) than males relative to body size.

Because humans, who are bipeds, are thought to be under selection for smaller biacetabular breadths and distances between the sacroiliac and acetabular joints

(Washburn, 1950, 1960; Pinkerton, 1973; Zihlman, 1978; Ruff, 1995; Lovejoy, 2005), 
and because biiliac breadth is assumed to be under stabilizing selection in humans for thermoregulatory imperatives (Ruff, 1993), it is usually presumed that dimorphism in human pelves is due to an "obstetric dilemma" in females (Washburn, 1960). Because selection for obstetric imperatives in females should increase the size of the maternal birth canal (Berge et al., 1984; Tague and Lovejoy, 1986; Abitbol, 1987b; Hager, 1989; Rosenberg, 1992; Abitbol, 1996; Rosenberg and Trevathan, 2002), female pelvic form is hypothesized to represent a tradeoff between selection for obstetric demand and selection for locomotor efficiency and/or thermoregulation (Ruff, 1995; Lovejoy, 2005). Further, this "obstetric dilemma" is thought to alter the timing of birth so that humans have a truncated gestational period (and consequently give birth to neonates with a smaller proportion of brain growth completed) compared to other apes (Washburn, 1960; DeSilva and Lesnik, 2006) as a result of locomotor selection limiting the size of the birth canal. However, recent research indicates that the "obstetric dilemma" may not exist in humans. Wider interacetabular distances in females may not pose an energetic cost compared to males (Warrener et al., 2015), and it is possible that maternal metabolic constraints rather than pelvic constraints alter the timing of birth in humans (Dunsworth et al., 2012). Further, the prevalence of cephalopelvic disproportion (mismatch between the neonatal head and maternal birth canal) in modern humans might be the result of the transition to agricultural subsistence and increased nutrition during pregnancy (Wells et al., 2012) and therefore might be unrelated to the adoption of bipedalism (Wells et al., 2012; Warrener et al., 2015).

These results present a conundrum - if there is no "obstetric dilemma", why are human pelves so dimorphic? It is possible that dimorphism in the birth canal is simply 
the result of obstetric selection on females (rather than a "tradeoff" between obstetrics and locomotion in females). If this were the case, it would be expected that other primate taxa that give birth to large neonates relative to maternal body size should also exhibit dramatic dimorphism in the birth canal. Though humans are the only extant bipedal primates, large cephalopelvic proportions, in which neonatal head size is large compared to maternal pelvic diameters, are common among primates (Schultz, 1949; Leutenegger, 1970, 1974; Stoller, 1995), especially among monkeys (Table 2.1). In contrast, the living apes (with the exception of Homo and Hylobates) have small cephalopelvic proportions (Table 2.1; Schultz, 1949; Leutenegger, 1982). The variation in cephalopelvic proportions that exists across anthropoid primates presents an opportunity to test the hypothesis that the existence of large cephalopelvic proportions within anthropoid species results in dimorphism in the size or shape of the birth canal.

\section{a. Which aspects of birth canal morphology should be the target of obstetric selection?}

Obstetric selection should hypothetically influence birth canal size, shape, or a combination of both, leading to dimorphism in the size or shape of the female birth canal compared to the bony equivalent in males. Though males obviously do not possess a birth canal, they do possess a negative space of the pelvis which is the bony equivalent of the female birth canal. For brevity, the bony equivalent of the female birth canal that exists in males will be referred to as the "birth canal" in males (even though a neonate does not pass through this structure in males). 
It is possible that only birth canal size is the target of obstetric selection because obstetric selection should be concerned principally with creating adequate space for the passage a neonate (Tague, 1991). Birth canal size can be increased in females by selection for an isometrically bigger birth canal in females compared to males. Alternatively, selection to increase the circumference of one or more pelvic planes - the pelvic inlet, midplane, and/or outlet - in females compared to males would lead to dimorphism in the size of these planes.

It is also possible that obstetric selection modifies birth canal shape. If there is no change in the circumference of the birth canal planes (i.e., absolute size), selection for a rounder birth canal - in which the mediolateral diameters of the pelvic planes approximate or exceed the dorsoventral diameters - may facilitate birth, because a comparatively larger neonatal head can fit through a rounder canal compared to a more ellipsoid one. Such selection should correspond to a mediolaterally wider birth canal in females compared to males.

Males and females may also differ in the size and shape of the birth canal. For the birth canal, selection on size and selection on shape are related, such that any increase in the mediolateral or dorsoventral (or both) diameters of each plane increase the circumference of that plane. However, if only the mediolateral or dorsoventral diameter increases, the shape of the birth canal changes. An increase in only the mediolateral dimension of the birth canal leads to a rounder or more transversely oval canal, whereas an increase in only the dorsoventral dimension creates a more dorsoventrally ellipsoid canal. A concurrent increase in the mediolateral and dorsoventral diameters of the birth canal increases circumference, but it does not change 
the shape of the canal. Thus, the mediolateral and dorsoventral diameters of the birth canal represent both aspects of size and shape.

Because there are many ways to create an obstetrically-sufficient birth canal (selection on size, selection on shape, selection on both), and because size and shape differences between males and females can be achieved through selection for dimorphism in different parts of the pelvis (sacrum, pubis, ilium, ischium), it is possible that there is not one 'pattern of dimorphism' that characterizes all primates with large cephalopelvic proportions (if there is any dimorphism at all). Accordingly, it is important to consider both size and shape differences between males and females within each species rather than looking for patterns of dimorphism that characterize all species.

\section{b. Dimorphism in the Birth Canals of Non-Human Primates}

Pelvic dimorphism is present across many mammalian taxa regardless of relative neonatal size (Gardner, 1936; Dunmire, 1955; Crelin and Newton, 1969; Chapman et al., 1994; Tague, 2003; Berdnikovs et al., 2007; Schutz et al., 2009a; Schutz et al., 2009b; Wells et al., 2012). Accordingly, previous research has not demonstrated a clear association between dimorphism in the size or shape of the birth canal and obstetric demand across anthropoid primates (Schultz, 1949; Steudel, 1981a; Tague, 1991; 2005). Across primates, females exhibit relatively greater transverse diameters of the pelvic inlet compared to males controlling for overall body size regardless of cephalopelvic proportions (Schultz, 1949; Tague, 2005), and pubis length is also known to be dimorphic in anthropoid taxa, even those with small cephalopelvic proportions (Steudel, 
1981a). Furthermore, there does not appear to be one pattern of dimorphism that characterizes all species of primates that have large cephalopelvic proportions (Tague, 1991). Yet, though the presence of dimorphism (whether or not there are any differences between sexes) may not due only to obstetric demand, it is possible that the magnitude of dimorphism in birth canal size and shape (how different the sexes appear) is greater among taxa with large cephalopelvic proportions compared to those with small proportions (Ridley, 1995).

The observation of pelvic dimorphism among anthropoids with small cephalopelvic proportions has prompted the hypothesis that factors other than parturition, including potentially overall body size or body size dimorphism, might influence pelvic dimorphism in anthropoid primates (Schultz, 1949; Tague, 1991; 2005). In anthropoid species with high levels of body size dimorphism (even those with small cephalopelvic proportions), females have exceptionally large birth canals (Tague, 2005). Thus, it is possible that divergent selection pressures on body size dimorphism (which may be driven by sexual selection) and pelvic dimorphism (which may be driven by obstetric selection) leads to dissociation between body size dimorphism and pelvic dimorphism (Tague, 2000; 2005; Kurki, 2011). Further, it is possible that the magnitude of dimorphism in birth canal size is affected by both body size dimorphism and cephalopelvic proportions, such that species with both large cephalopelvic proportions and high levels of body size dimorphism also have the most dimorphic pelves.

Most previous research addresses dimorphism in the size of the birth canal planes or individual bony elements in primates (Washburn,1948; Schultz, 1949; 
Leutenegger, 1974; Mobb and Wood, 1977; Steudel, 1981a; Ridley, 1995; Tague, 2005) rather than dimorphism in birth canal shape. The little research that does exist on dimorphism in birth canal shape in anthropoids indicates there is not a clear association between dimorphism in birth canal shape and cephalopelvic proportions in primates (Tague, 1991). However, it is possible that previous methods used to quantify shape differences in the planes of the birth canal between males and females (using ratios of mediolateral to anteroposterior diameters of each birth canal plane; Tague, 1991) do not capture birth canal shape differences between the sexes. For instance, both monkeys and apes have significant dimorphism in the transverse diameters of the birth canal, but only monkeys (not apes) also have high levels of dimorphism in the anteroposterior diameters of the inlet (Tague, 1991). Thus, when using a ratio of transverse to mediolateral birth canal dimensions to quantify shape in apes, females have "rounder" birth canals than in males (Schultz, 1949; Tague, 1991) due to dimorphism in only the mediolateral dimensions of the birth canal, while in monkeys, the sexes are not different for birth canal shapes (Schultz, 1949; Tague, 1991) because female monkeys have larger mediolateral and dorsoventral dimensions of the birth canal than males (Tague, 1991). Furthermore, using ratios of linear distances to quantify birth canal shape also does not allow examination of how dimorphism in the individual bony elements- ilium ischium, pubis, sacrum - contributes to dimorphism in the size and shape of the birth canal. Because dimorphism in the spatial relationships among the bony elements of the pelvic girdle are what actually cause dimorphism in the size and the shape of the birth canal planes, considering three dimensional birth canal morphology is necessary (Moffett et al., 2013; Lycett and von Cramon-Taubdel, 2013). 
Similar to dimorphism in size, it is possible that there is dimorphism in birth canal shape across anthropoid taxa regardless of cephalopelvic proportions, but that the magnitude of dimorphism is greater in species with large proportions. If this is the case, it would be expected that there is little overlap in birth canal shape between males and females in species with large cephalopelvic proportions, but more overlap between sexes in species with small proportions (because there is theoretically low obstetric selection on birth canal form in these females). However, this hypothesis has not been tested across anthropoid primates.

\section{HYPOTHESES}

The goal of the current study is to determine how obstetric demand and cephalopelvic proportions influence dimorphism in the size and shape of the birth canal across anthropoids. This study tests the following hypotheses:

Hypothesis 1: Dimorphism in birth canal size is due to obstetric selection.

Prediction 1a: In species with large cephalopelvic proportions, females will have larger birth canals overall (quantified as centroid size of birth canal) compared to males controlling for body size, and this will not be the case for species with small proportions.

Prediction 1b: In species with large cephalopelvic proportions, females will have larger circumferences of the inlet, midplane, and outlet compared to males controlling for body size, and this will not be the case in species with small proportions. 
Prediction 1c: In species with large cephalopelvic proportions, females will have larger mediolateral and dorsoventral dimensions of the inlet, midplane, and outlet compared to males controlling for body size, and this will not be the case in species with small proportions.

Prediction 1d: If dimorphism in birth canal size is found in species with both large and small cephalopelvic proportions, there will be a greater magnitude of dimorphism in birth canal dimensions among species with large cephalopelvic proportions compared with species having small proportions.

Hypothesis 2: Dimorphism in birth canal shape is due to obstetric selection.

Prediction 2a: In species with large cephalopelvic proportions, males and females are dimorphic for the overall shape of the birth canal and this is not true in species with small proportions.

Prediction 2b: In species with large cephalopelvic proportions, females have rounder inlets, midplanes, and outlets compared to males, and this is not true in species with small proportions.

Prediction 2c: If dimorphism in birth canal shape is not restricted to species with large cephalopelvic proportions, there is a greater magnitude of dimorphism (i.e., less shape overlap) in birth canal shape among species with large cephalopelvic proportions compared with species having small proportions. 


\section{MATERIALS AND METHODS}

\section{a. Samples}

Species were chosen for this study based on: 1) existing data on cephalopelvic proportions (Schultz, 1949; Leutenegger, 1982; Stoller, 1995) and 2) adequate samples available for shape analysis. Sample size for morphometric comparisons of pelvic dimensions was determined using a power analysis, conducted in $\mathrm{R}$ using the package 'pwr', of 5 male and 5 female specimens of $H$. lar. Results indicated that sample size should equal at least 12 individuals per species, per sex to achieve power of 0.90 for detecting pelvic shape differences between sexes using Student's t-tests.

Adult male and female pelves $(\mathrm{n}=920)$ from 9 anthropoid species (Table 2.1) constituted the sample for the current study. Skeletal maturity was determined using fusion of the iliac crest and ischial tuberosity. Of the species included in this study, 6 have large cephalopelvic proportions [Homo sapiens $(\mathrm{n}=156)$, Hylobates lar $(\mathrm{n}=112)$, Macaca mulatta $(\mathrm{n}=187)$, Nasalis larvatus $(\mathrm{n}=33)$, Papio anubis $(\mathrm{n}=21)$, Saimiri sciureus $(\mathrm{n}=153)$ ] and 3 have small proportions [Alouatta seniculus $(\mathrm{n}=38)$, Gorilla gorilla $(\mathrm{n}=106)$, Pan troglodytes $(\mathrm{n}=114)]$. This sample comprises a range of cephalopelvic proportions, phylogenetic affinity, overall body size, and body size dimorphism among extant primates. While phylogeny may influence birth canal morphology, samples for too few taxa exist to achieve sufficient power (Blomberg et al., 2003) due to a dearth of information on cephalopelvic proportions among many primates. However, including species with large and small cephalopelvic proportions within hominoids [Homo sapiens and Hylobates lar (large proportions); Gorilla gorilla and Pan troglodytes (small proportions)] and platyrrhines [Saimiri sciureus (large 
proportions) and Alouatta seniculus (small proportions)] provided a set of comparisons with which to consider phylogeny and develop hypotheses for robust testing if a broader range of taxa become available. Similarly, locomotor repertoire may be an important factor, but locomotion is completely confounded with phylogeny in anthropoids. Thus, no formal tests considering locomotion were performed.

\section{b. Landmarks}

Paired ossa coxae and sacra were articulated with isobutylene-isoprene polymer adhesive mounting putty and rubber bands (Tague, 1991) without compensating for cartilage (Tague, 1986; Grabowski, 2013). 5 bilateral and 5 midline landmarks representing the birth canal are then collected with a Microscribe G2X digitizer. Landmarks (Table 2.2; Figure 2.1) include sacroiliac joint at arcuate line, lateral arcuate line, arcuate line at acetabulum, superior pubic symphysis, and anterior S1 body (inlet); the ischial spine, inferior pubic symphysis, and inferior S1 body (midplane); and the posterior ischial tuberosity, inferior pubic symphysis, and inferior sacrum (outlet) (Figure 2.1). In humans, the dorsal-most point of the midplane is typically measured from the anterior S4 vertebrae (Tague, 1992, Brown, 2007; Kurki, 2007, 2011). However, modal numbers of sacral vertebrae in great apes, $\mathrm{H}$. lar, and monkeys are 5, 4, and 3, respectively (Schultz, 1936). Therefore, for this study, the anterior inferior S1 vertebral body was chosen as the posterior landmark for the midplane so that this point could be collected at the same point on all specimens. 
Error in digitizing was assessed using repeated measures analysis (Kohn, 1992). One pelvis of $P$. troglodytes (large body size) and one pelvis of $S$. sciureus (small body size) was digitized, and all landmarks were collected on each specimen ten times. For this study, repeated measures are used to quantify landmark repeatability (Kohn, 1995). Repeated measures analysis yielded error averages for all landmarks equal to or less than $0.16 \mathrm{~mm}$ for Pan. troglodytes and $0.19 \mathrm{~mm}$ for Saimiri sciureus. These averages were less than $0.002 \%$ of centroid size for both specimens, and error was equally distributed across the three axes. This analysis indicated that all landmarks can be collected with high repeatability regardless of the size of specimens.

\section{c. Testing Hypothesis 1}

To test the hypothesis that dimorphism in birth canal size is due to large cephalopelvic proportions, centroid size of the birth canal (a measure of overall birth canal size) was calculated as the square root of the sum of the squared distances between all birth canal landmarks and the centroid of the configuration (Bookstein, 1991) for each individual. Centroid size was chosen to represent the size of the entire birth canal. Circumferences of the inlet, midplane, and outlet were quantified following Tague (1991) and secondarily calculated from landmark data following the formulas in Table 2.3 (illustrated in Figure 2.2). Dorsoventral and mediolateral diameters of the inlet, midplane, and outlet were also quantified following Tague (1991), and each linear distance was secondarily calculated from landmark data following the formulas in Table 2.3 (also illustrated in Figure 2.2). The circumferences and mediolateral/anteroposterior dimensions of each plane quantified the size of each plane of the birth canal. 
Absolute measures of birth canal size are of limited utility for evaluating sexual dimorphism, because absolute measures are expected to be sexually dimorphic in species in which males have much larger body size than females - for example Gorilla gorilla and Nasalis larvatus (Table 2.1). However, the fact that males have absolutely larger measurements in such species does not preclude the possibility of females possessing relatively larger birth canals when controlled for body size, which is the question being investigated here. Thus, ratios of birth canal dimensions against body size proxies (see below) were computed in order to compare the size of the birth canal between males and females. Ratios were calculated as (absolute birth canal dimension)/(S1 body breadth) for each birth canal size variable for each individual (Moffett et al., 2013). These ratios represent the relative size (controlling for body size) of each birth canal size variable.

Performing statistical analyses on ratios can lead to spurious results, especially if there is non-isometric scaling among variables (Atchley et al., 1976), and any differences between sexes in relative pelvic size may simply be due to allometric scaling between pelvic size variables and S1 body breadth. Therefore, any measure of body size chosen for the present study should ideally scale isometrically with all measures of birth canal size. The most appropriate measure of body size is body mass (Smith and Jungers, 1997), but individual body mass data does not exist for most specimens included in this study. Previous authors have used the geometric mean of pelvic linear data as a proxy for body size in anthropoids (Steudel, 1981a; Li, 2002), but this measure of body size is inappropriate for the present study because it is not independent of the measures of birth canal size analyzed. This study therefore used one 
body size proxy that is independent of the aspects of pelvic form being analyzed here: transverse breadth of the first sacral (S1) vertebral body. S1 body breadth (also called lumbosacral articulation; Jungers, 1988) is strongly correlated with body mass across primates (Jungers, 1988; 1991). It should be noted that previous authors have found that many postcranial skeletal indicators of body size produce inaccurate estimates of body size dimorphism in primates compared to cranial indicators (Plavcan, 2003). However, body mass was unavailable for most specimens included, and many pelves were not paired with associated crania.

Next it was necessary to assess whether the pelvic size variables examined in this study scale isometrically with S1 body breadth. It is ideal to perform regressions on the smallest subgroups (individual sexes within individual species) because this ensures similar scaling between sexes within individual taxa. However, for some taxa, there was not enough variation in body size within sexes, or even within species - for example, in Saimiri sciureus and Hylobates lar - to obtain a strong correlation between variables. Therefore, regressions were performed across (a) all taxa included in order to obtain scaling of birth canal dimensions across a wide range of body sizes and (b) within phylogenetic groups (i.e., apes and monkeys) to determine whether scaling patterns are similar across these groups. Regressions were performed only on male specimens in order to determine allometric scaling of birth canal dimensions (used here to mean dimensions of the pelvis that in females contribute the birth canal, as of course males do not have birth canals) when birth is not a factor. Natural logged birth canal dimensions were regressed against natural logged body size proxies using reduced major axis regression (Harvey and Pagel, 1991; Warton et al., 2006; Smith, 2009; but see Hansen 
and Bartoszek, 2012) in R using the package 'Imodel2'. Strength of the relationship between body size proxies and birth canal dimensions was determined using r-squared values. Isometry was determined using $95 \%$ confidence intervals, such that confidence intervals which include a slope of 1 are considered isometric.

For pelvic size variables that scale isometrically with $\mathrm{S} 1$ body breadth, ratios of these variables with $\mathrm{S} 1$ body breadth may be used to compare relative size between males and females. Within each species, birth canal size ratios were statistically compared between males and females within each species using a two-tailed Student's t-test at $\alpha=0.035$ (see justification for $\alpha$-level below). If females have relatively larger birth canals than males controlling for overall body size, this should manifest itself as statistically larger ratios (of pelvic size variables divided by $\mathrm{S} 1$ body breadth) in females.

For pelvic size variables which scale allometrically with $\mathrm{S} 1$ body breadth, ratios may be unreliable. Therefore, within each species, residuals from the male-only taxonwide regression (i.e., within apes or within monkeys) between each pelvic size variable and S1 body breadth were calculated, and residuals were statistically compared between males and females using Student's t-tests. If females have relatively large birth canals controlling for body size compared to males, this should manifest itself as larger and more positive residuals from the taxon-wide regression in females, but not in males. Furthermore, if dimorphism in birth canal size is due to large cephalopelvic proportions, residuals should be statistically different in males and females in species with cephalopelvic proportions, but not different in males and females in species with small proportions. 
It is possible that dimorphism in birth canal size (i.e., significant differences in birth canal dimensions) is present across taxa regardless of cephalopelvic proportions, but that there is a larger magnitude of dimorphism (greater difference in birth canal size) among species with large cephalopelvic proportions. Therefore, for each birth canal dimension, a dimorphism index was created within each species to facilitate comparison of the magnitude of dimorphism (i.e. the magnitude of difference between males and females with species) among species. Each dimorphism index was calculated as:

$$
\text { dimorphism index }=\left[\frac{\text { female mean }}{\text { male mean }}\right] * 100
$$

such that the further the value of the dimorphism index is from 1, the larger the difference between males and females for a given birth canal dimension. For each birth canal dimension, dimorphism indices for species were ranked from largest (females larger than males) to smallest (little difference between the sexes) in order to allow comparison of the magnitude of dimorphism in birth canal size.

Hypothesis 1 was considered falsified if within each species having large cephalopelvic proportions (a) females do not exhibit statistically larger centroid sizes of their birth canals compared to males controlling for overall body sizes; (b) females do not exhibit larger circumferences of the pelvic inlet, midplane and outlet than males controlling for overall body sizes; (c) females do not exhibit larger mediolateral or transverse diameters of the pelvic inlet, midplane and outlet than males controlling for overall body size; or (d) there is not a greater magnitude of dimorphism in species with large cephalopelvic proportions compared to species with small proportions. 


\section{d. Testing Hypothesis 2}

To test the hypothesis that dimorphism in overall birth canal shape is due to large cephalopelvic proportions, it was first necessary to quantify the overall shape of the birth canal (landmarks used to quantify birth canal shapes shown in Figure 2.1) were quantified using 3-dimensional geometric morphometrics. Original landmark data were scaled, translated, and rotated using generalized Procrustes analysis. Principal components analysis (PCA), an eigenanalysis-based tool for ordination, was then employed to compare birth canal shapes within each species. To obtain shape coordinates, all size information is removed by scaling (Zelditch et al., 2004); therefore, principal components scores reflect size-free shape data. All PCAs were conducted using Morphologika 2.5@ software. To determine which successive principal components (PCs) significantly contributed to shape variability within each species, scree plots were used to visualize the inflection points of variance explained by successive PCs (Zelditch et al., 2004). For scree plots which did not have an obvious inflection point, PCs which represented more than $10 \%$ of total shape variance were included in the analysis, as these components can be said to contribute to biologically meaningful variation (Zelditch et al., 2004).

Discriminant Function Analysis (DFA) was employed to determine whether males and females are significantly different for birth canal shape, quantified as principal components scores of birth canal shape within each species. All Discriminant Function Analyses were performed in R using the package 'MASS'. However, sample 
size effects are known to affect error rates in DFA (Lachenbruch, 1968). Power analysis indicated that in order to achieve power of 0.90 for detecting pelvic shape differences between sexes using DFA at effect size of 18 individuals per species per sex. Therefore, this method of determining sex differences in overall birth canal shape could lead to an increased probability of Type II error for species represented by small sample sizes, specifically Papio anubis (Female $\mathrm{n}=5$ ) and Nasalis larvatus $($ Female $\mathrm{n}=16)$. Therefore, as an additional strategy to quantify the degree of overlap in birth canal shapes within each species in a manner that is not sensitive to sample size, a polygon was placed around the male shape distribution (which theoretically represents the shape of the structure when there is no obstetric selection) in the principal components plot. Then, the proportion of females that fell within the male distribution was calculated.

In order to determine which Principal Components of birth canal shape males and females separate along, Student's t-tests were performed on raw Principal Component scores between males and females within each species for all PCs that encompass significant shape variation (i.e., $>10 \%$ of intraspecies shape variation) . All t-tests of differences in means between male and female PC scores within species were performed in R. Visualization of shape differences between males and females within each species was facilitated using wireframes created in Morphologika.

While PCA is useful for determining whether there is dimorphism in birth canal shape within species, these techniques do not provide details of which elements of the birth canal actually differ between males and females. Thus, in order to determine precisely which regions of the birth canal differ between males and females, Euclidean Distance Matrix Analysis (EDMA) was used to localize shape differences between 
males and females (Lele and Richstmeier, 1991; 1995; 2001; following Brown, 2011). EDMA's FORM analysis allows quantification of the linear distances (for this study, interlandmark distances) which significantly contribute to overall shape differences between samples using a bootstrap approach. Significance of each interlandmark distance was determined using bootstrapped confidence intervals. The FORM procedure also estimates the difference between mean forms (i.e., overall form differences between groups). For differences between mean forms using EDMA's FORM procedure, all p-values $<0.035$ were considered significant (see justification for $\alpha$-level below). The FORM procedure was performed on both unscaled data as well as data scaled to each individual's geometric mean (in order to obtain shape information controlling for size; Lele and Richstmeier, 1991; 1995). All FORM analyses were performed in WinEDMA Version 1.0.1 Beta (Cole, 2002).

"Roundness" of the birth canal planes between males and females was also quantified within each species. An index was created following Tague (1991) in which the mediolateral dimension of each birth canal plane (secondarily calculated from landmark data; Figure 2.2; Table 2.3) was divided by the dorsoventral dimension of that plane (secondarily calculated from landmark data; Figure 2.2; Table 2.3) for each individual. Thus, in this ratio, a larger value (close to or exceeding one) indicates a transversely rounder inlet, midplane, or outlet whereas a smaller ratio (less than one) indicates a more ellipsoid plane. Ratios calculated for the shape of the inlet, midplane, and outlet cannot be statistically compared between males and females because there are different allometric scaling relationships for mediolateral and dorsoventral diameters of the birth canal with body size (see Results below and Table 2.5). However, 
these ratios do provide information about the relative roundness or ellipsoid-shape of each pelvic plane in females versus males.

The predictions of Hypothesis 2 were considered falsified if in species with large cephalopelvic proportions (a) there is significant overlap in overall birth canal shape between males and females or (b)females do not have rounder birth canal shapes compared to males.

\section{e. Controlling for multiple comparisons}

A potential issue with performing a large number of statistical analyses is the increasing probability of false positives, or Type I Errors, with the increasing number of tests. In this study, multiple statistical procedures are performed on related data; therefore, using an $\alpha$-level of 0.05 would lead to an increased probability to Type I Error. Therefore, in order to control for multiple comparisons in this study, a False Discovery Rate (FDR) procedure (Benjamini and Hochberg, 1995) was conducted on all p-values obtained from this study (i.e., all those obtained in Student's t-tests of absolute and relative birth canal dimensions, DFA, Student's t-tests of PC scores, and EDMA's FORM tests) using the R package 'qvalue' (Storey, 2015). Unlike a Bonferroni correction, FDR is not excessively conservative in which results are considered significant. The FDR procedure indicated that in order to maintain a false discovery rate of $5 \%, \alpha$-level should be set at 0.035 . Therefore, unadjusted p-values are reported in the text, but only those p-values less than 0.035 were considered significant. 


\section{RESULTS}

\section{a. Allometry in Birth Canal Size Variables}

Allometric scaling of birth canal dimensions with S1 body breadth is presented in Table 2.4 and Table 2.5. Birth canal centroid size and circumferences of the inlet, midplane, and outlet scale with isometry with S1 body breadth both across all anthropoid taxa and within apes and monkeys (Table 2.4). Therefore, differences between males and females in the ratio of birth canal centroid size and/or circumferences of the inlet, midplane and outlet against S1 body breadth are likely not due to allometric scaling. Within apes and monkeys, mediolateral inlet diameter and dorsoventral inlet diameters also scale isometrically with S1 body breadth (Table 2.5), meaning that any differences between males and females within taxa in either mediolateral or dorsoventral inlet diameter is likely not due to allometry.

Mediolateral midplane diameter scales isometrically with S1 body breadth in monkeys but very slightly negatively allometrically in apes (Table 2.5), which indicates that differences between males and females for relative mediolateral midplane diameter is likely not due to allometric scaling in monkeys or apes with low levels of body size dimorphism (H. sapiens, Hy. lar, P. troglodytes). However, mediolateral midplane diameter might be relatively larger in females than males in ape species with extremely high levels of body size dimorphism (G. gorilla) simply due to allometric scaling of mediolateral midplane diameter with $\mathrm{S} 1$ body breadth in apes.

Dorsoventral diameters of the midplane scale isometrically with S1 body breadth in apes, but positively allometrically in monkeys (Table 2.5). This indicates that 
among apes, any dimorphism in relative dorsoventral midplane diameters is likely not due to allometric scaling. However, in monkey taxa with extremely high levels of body size dimorphism ( $N$. larvatus and P. anubis), it would be expected that males would be relatively larger than females for dorsoventral midplane diameters, but this should not be the case in monkey taxa with low levels of body size dimorphism (Macaca mulatta, Saimiri sciureus and Alouatta seniculus).

Mediolateral outlet diameter scaled with strong positive allometry in apes but negative allometry in monkeys (Table 2.5). Therefore, it would be expected that among ape taxa which are highly dimorphic in body size, males are relatively larger than females for outlet diameter due to allometric scaling, but this should not be the case in ape taxa with low levels of body size dimorphism. In contrast, in monkey taxa with high levels of body size dimorphism, females should exhibit relatively larger mediolateral outlet diameters compared to males, but this should not be the case in monkey taxa which are less dimorphic in body size.

Dorsoventral outlet diameter scales with very slight negative allometry in apes but strong positive allometry in monkeys (Table 2.5). Based on these scaling relationships, it is expected that in apes with very high levels of body size dimorphism females are relatively larger than males for dorsoventral outlet diameter, but this should not be true in apes with lower levels of body size dimorphism. In monkeys with high levels of body size dimorphism, males should be relatively much larger than females for dorsoventral outlet diameters, but in less body size dimorphic taxa, scaling should not affect dimorphism in dorsoventral outlet diameter. 


\section{a. Is dimorphism in birth canal size within species is due to obstetric selection?}

This hypothesis first predicts that in species with large cephalopelvic proportions, females will have a larger overall birth canal compared to males controlling for body size, but this will not be true in species with small proportions. The results of this study (Tables 2.6-2.8) do not support this hypothesis. The presence of dimorphism in birth canal centroid size (controlling for body size) is not restricted to species with large cephalopelvic proportions. Across species with large cephalopelvic proportions (Homo sapiens, Hylobates lar, Macaca mulatta, Nasalis larvatus, Papio anubis, and Saimiri sciureus), females do indeed have larger centroid sizes of the birth canal than males controlling for overall body size (all p-values $<0.001$; Tables 2.6 and 2.8). However, in Pan troglodytes (small proportions), females are also relatively larger than males for birth canal centroid size controlling for body size $(\mathrm{p}<0.001$; dimorphism index $=106$; Table 2.6). These results are not due to allometric scaling of birth canal centroid size with S1 body breadth, because birth canal centroid size scales isometrically with $\mathrm{S} 1$ body breadth across apes and monkeys, and t-tests performed on residuals of the RMA regression between pelvic centroid size and S1 body breadth are identical to the results obtained using ratios (Table 2.8).

Hypothesis 1 also predicts that in species with large cephalopelvic proportions females have relatively larger circumferences of the pelvic inlet, midplane, and outlet than males, but that this is not the case in species with small proportions. This prediction is also not supported because the presence of dimorphism in circumferences of the inlet, midplane, and outlet (controlling for body size) is not restricted to species with large cephalopelvic proportions (Table 2.6). Females are relatively larger than 
males (controlling for body size) for the circumferences of the inlet (all p-values $<0.001$ ), midplane (all p-values <0.003), and outlet (all p-values <0.006; Table 2.6) in taxa with large cephalopelvic proportions. However, in Gorilla gorilla and Pan troglodytes (both with small proportions), females are relatively larger than males for pelvic inlet and midplane circumferences (all p-values $<0.001$ ), and in P. troglodytes females are also relatively larger than males for outlet circumference $(\mathrm{p}<0.001)$. Because all birth canal circumferences scale isometrically with S1 body breadth across apes and monkeys, these results are not due to allometric scaling (Table 2.8).

Next, Hypothesis 1 predicts that in species with large cephalopelvic proportions, females have larger mediolateral and dorsoventral dimensions of the inlet, midplane, and outlet compared to males controlling for body size, and this is not the case in species with small proportions. This prediction is also not supported. Dimorphism in the relative mediolateral and dorsoventral diameters of the inlet, midplane, and outlet are not restricted to species with large cephalopelvic proportions (Table 2.7). The transverse diameter of the inlet is relatively larger in females than males (controlling for body size) in Homo sapiens, Hylobates lar, Nasalis larvatus, and Saimiri sciures (all having large cephalopelvic proportions) as well as Gorilla gorilla and Pan troglodytes (small proportions; Table 2.7). This result is not due to allometric scaling (Table 2.8), because mediolateral inlet diameter scales isometrically with $\mathrm{S} 1$ body breadth in apes and monkeys. The transverse diameter of the midplane is also relatively larger in females than males in $H$. sapiens, Hy. lar, N. larvatus, S. sciureus, G. gorilla, and $P$. troglodytes (Table 2.7) and results obtained using residuals (Table 2.8) indicate that females have relatively larger transverse midplane diameters than males in M. mulatta 
and $P$. anubis. The transverse diameter of the outlet (which scales positively allometrically with $\mathrm{S} 1$ body breadth in apes and negatively in monkeys; Table 2.5 ) is relatively larger in females than males in all taxa with large cephalopelvic proportions as well as $P$. troglodytes (small proportions). .

The dorsoventral diameters of the inlet and midplane are relatively larger in females than males in all taxa with large proportions (Table 2.8), but also in $P$. troglodytes (small proportions). The dorsoventral diameter of the outlet is relatively larger in females among some taxa with large cephalopelvic proportions (H. sapiens, $H$. lar, M. mulatta; Table 2.8) as well as in G. gorilla and P. troglodytes (small proportions).

Even though all species (regardless of cephalopelvic proportions) exhibit some dimorphism in birth canal size, the magnitude of dimorphism in birth canal centroid size and circumferences of the pelvic inlet, midplane, and outlet generally differ between taxa with small and large cephalopelvic proportions (Table 2.9), supporting Hypothesis 1 Prediction $1 \mathrm{~d}$. In species with large proportions, there is a high magnitude of dimorphism in birth canal centroid size [dimorphism index ranging from 105 (females slightly larger than males) in H. lar and M. mulatta to 130 (females much larger than males) in N. larvatus; Table 2.9] compared to species with small proportions, in which dimorphism indices are lower (ranging from 100 in A. seniculus to 106 in $P$. troglodytes). These results indicate that species with large cephalopelvic proportions do tend to be more dimorphic than those without, though there are exceptions (for example, $P$. troglodytes). Among species with large cephalopelvic proportions, there also tend to be higher dimorphism indices for the relative circumferences of the pelvic 
inlet $($ index range $=108-122)$, midplane $($ range $=105-117)$, and outlet $($ range $=106$ 119) compared to species with small proportions for the inlet (range $=100-109)$, midplane (range $=103-107)$, and outlet $(100-107)$.

Though females do have relatively larger birth canals that males, in most species (all those studied here except Alouatta seniculus), there is a great degree of overlap in birth canal size across most taxa (except for Nasalis larvatus and Papio anubis; see Appendix A). Thus, differences in birth canal size may not be observable at small sample sizes in many taxa. This is especially important to consider when examining dimorphism in birth canal size in fossil specimens, which will be represented by smaller sample sizes than the species included here.

Though dimorphism in birth canal size is not restricted to species with large cephalopelvic proportions, species with large cephalopelvic proportions do tend to be more dimorphic in birth canal centroid size and circumferences of the inlet, midplane, and outlet than species with small cephalopelvic proportions. So although the data reject the hypothesis that cephalopelvic proportions are the determinant of pelvic dimorphism, they suggest that cephalopelvic proportions influence dimorphism, and result in more dimorphic pelves in taxa with relatively larger neonates.

\section{b. Is dimorphism in birth canal shape within species is due to obstetric selection?}

Hypothesis 2 Prediction a posits that males and females will be dimorphic for the overall shape of the birth canal in species with large cephalopelvic proportions but 
not in those with small proportions. This hypothesis is not supported by these analyses. Discriminant Function Analysis indicates that in both taxa with large and small cephalopelvic proportions, the sexes differ in the overall shape of the birth canal (Table 2.10) Among all taxa included in this study, males and females significantly separate in shape space along one or more Principal Components (Table 2.11), and EDMA's FORM results likewise indicate that the sexes are significantly different for overall birth canal shape in all species except $A$. seniculus (Table 2.12).

Hypothesis 2 also predicts that in species with large cephalopelvic proportions, females have a "rounder" birth canal compared to the bony equivalent in males, but this is not true in species with small proportions. The data do not support this hypothesis, either (Table 2.13). Only one species with large cephalopelvic proportions (Hylobates lar) has females which have rounder pelvic inlets than males, and one species with small cephalopelvic proportions (Pan troglodytes) also has females which exhibit rounder inlets compared to males. Females have rounder pelvic midplanes compared to males in all taxa (regardless of cephalopelvic proportions) except for Saimiri sciureus (which notably has the largest cephalopelvic proportions). Females do have rounder pelvic outlets than males in all species with large proportions except Saimiri sciureus, but in P. troglodytes (small proportions) females also have rounder outlets compared to males (Table 2.13).

Finally, Hypothesis 2 predicts that even if there is some shape dimorphism in all taxa, it will be greater species with large cephalopelvic proportions. Data show that there is less overlap between males and females in birth canal shapes among species with large cephalopelvic proportions (ranging from $0 \%$ to $39 \%$ overlap between sexes 
for birth canal form; Table 2.14) compared to species with small proportions (ranging from $38 \%$ to $79 \%$ overlap between sexes for birth canal form; Table 2.14; Figure 2.3).

Though dimorphism in birth canal form is not restricted to species with large cephalopelvic proportions, the magnitude of dimorphism in birth canal shape is greater among species with large cephalopelvic proportions compared to species with small cephalopelvic proportions.

\section{c. Interspecific Differences in Pattern of Dimorphism}

All species included in this study exhibit dimorphism for some aspects of birth canal size and shape, but each species in a slightly different way.

\section{Large Cephalopelvic Proportions}

\section{Homo sapiens:}

Birth Canal Size: In H. sapiens, females are relatively larger than males for relative birth canal centroid size (Table 2.6), and females are absolutely and relatively larger for the circumferences of the inlet, midplane, and outlet (Table 2.6). Females achieve these spacious birth canal planes by having absolutely larger transverse midplane and outlet diameters and dorsoventral inlet and outlet diameters, and females are also relatively larger for the transverse inlet diameter and dorsoventral midplane diameter (Table 2.7).

Birth Canal Shape: Homo sapiens males and females exhibit dramatic differences in birth canal shape (Figures 2.4a and 2.4b; Tables 2.10-2.12). Only ten percent of females fall within the male distribution for birth canal form (Figure 2.4a; 
Table 2.14). Females have longer and more coronally-oriented pubes, shorter pubic symphyses and sacra, more dorsally-angled caudal sacra and laterally flaring ischial spines and ischial tuberosities, and relatively wider sacra (Figures 2.4a and 2.4b). Males are relatively larger for pubic symphysis length, sacral length, ischial length, and length of the lateral linea terminalis (Figure 2.4b).

\section{Hylobates lar}

Birth Canal Size: In H. lar, sexes are monomorphic for body size (Table 2.1), but females are absolutely and relatively larger than males for centroid size of the birth canal and circumferences of the inlet, midplane, and outlet (Table 2.7). Females achieve these larger birth canals with absolutely larger transverse and dorsoventral diameters of the inlet, midplane, and outlet (Table 2.7).

Birth Canal Shape: Birth canal shape is unambiguously dimorphic in some dimensions in this species (Figures 2.5a and 2.5b: Tables 2.10-2.12), but there is more overlap in birth canal shapes between sexes in $H$. lar compared to H. sapiens $-33 \%$ of females fall within the male distribution (Figure 2.5a; Table 2.14). Males have a more rostrocaudally elongated canal due to a higher position of the sacra, longer sacra, longer ischia, and longer pubic symphyses. Females have mediolaterally expanded birth canals as a result of relatively longer pubes and wider sacra (Figure 2.5b).

\section{Macaca mulatta}

Birth Canal Size: In contrast to $H$. sapiens, $M$. mulatta has large cephalopelvic proportions but also greater body size dimorphism (Table 2.1), so males are absolutely larger than females for birth canal centroid size and circumferences of the inlet and 
midplane (Table 2.6), but relative to body size females have larger birth canal centroid size and circumferences of all three planes (Table 2.6). Females achieve these relatively larger birth canal circumferences by being relatively larger for the mediolateral and dorsoventral diameters of the inlet and outlet (Table 2.7). Midplane diameters are not significantly dimorphic, but are slightly larger in females, contributing to dimorphism in relative midplane circumference.

Birth Canal Shape: Birth canal shape is significantly and dramatically dimorphic in M. mulatta (Tables 2.10-2.12; Figures 2.6a and 2.6b). Only ten percent of females fall within the male distribution for birth canal form (Figure 2.6a; Table 2.14). Females exhibit mediolaterally expanded inlets and outlets due to their relatively longer pubes, mediolaterally expanded sacral alae, and laterally flared ischia. Males have a rostrocaudally expanded birth canal due to relatively longer sacra, ischia, and pubic symphyses (Figure 2.6b).

\section{Nasalis larvatus}

Birth Canal Size: In N. larvatus, males have absolutely larger midplane and outlet circumferences (but not inlet circumferences) than do females (Table 2.6), but females are relatively larger in centroid sizes and all birth canal circumferences (Table 2.6). Females achieve these relatively larger birth canals by having relatively larger mediolateral and dorsoventral diameters of all birth canal planes (Table 2.7).

Birth Canal Shape: Males and females clearly exhibit differences in birth canal shapes (Tables 2.11 - 2.12; Figures 2.7a and 2.7b). Though DFA indicates that there are no significant differences between sexes (Table 2.10), this result is likely due 
to small sample size, because there is no overlap between the sexes for birth canal form in this species (Figure 2.7a; Table 2.14). Females have elongated pubic bones (Figures 2.7a and 2.7b). In contrast, males have a much narrower birth canal due to longer and medially-projecting dorsal ischia, and relatively longer pubic symphyses and sacra (Figure 2.7b).

\section{Papio anubis}

Birth Canal Size: In this species, males are absolutely larger than females for midplane circumference, but sexes do not differ in any other absolute measure (Table 2.6). However, females are relatively larger for birth canal centroid size and all circumferences (Table 2.6). Females have larger mediolateral and dorsoventral dimensions of the inlet (Table 2.7), and although slightly larger across midplane and outlet these differences between sexes are not significant, possibly due to the small sample size available for this taxon). Still, these differences contribute to dimorphism in relative midplane and outlet circumference (Table 2.6).

Birth Canal Shape: In P. anubis, sexes have significant differences in birth canal shape (Tables 2.10-2.12; Figures 2.8a and 2.8b). Though DFA indicates that there are no significant differences between sexes for shape (Table 2.13), this result is likely due to small sample size, because there is no overlap between the sexes for shape in $P$. anubis (Figure 2.8a; Table 2.14). Female $P$. anubis have laterally flaring inferior ischia due to more coronally-oriented inferior pubic rami in females (Figures 2.8a and 2.8b). Conversely, males have absolutely (and relatively) longer pubic symphyses and ischia (Figure 2.8b). 


\section{Saimiri sciureus}

Birth Canal Size: Females are absolutely (and relatively) larger than males for birth canal centroid size and circumferences of the inlet, midplane, and outlet (Table 2.6). Females achieve these large birth canals by being absolutely and relatively larger for the transverse diameters of the inlet and midplane and all dorsoventral diameters of the inlet, midplane and outlet. Females are also relatively (but not absolutely) larger for the transverse diameter of the outlet (Table 2.7).

Birth Canal Shape: Males and females exhibit dramatic and significant differences in birth canal shapes (Tables 2.10-2.12; Figures 2.9a and 2.9b). Females have relatively shorter and wider sacra, more dorsally-angled inferior sacra, longer pubes, a more inferior position of the inferior and superior pubic rami (Figure 2.9b). Males have relatively longer pubic symphyses, ischia, and sacra compared to females. Compared to other large cephalopelvic proportion species, there is considerable overlap in shape between the sexes in S. sciureus (39\%, Figure 2.9a; Table 2.14).

\section{Small Cephalopelvic Proportions:}

\section{Gorilla gorilla}

Birth Canal Size: In G. gorilla, a species with high levels of body size dimorphism (Table 2.1), males are absolutely larger than females for birth canal centroid size and circumferences of the inlet, midplane, and outlet (Table 2.6), yet females are relatively larger for all of these variables. Females are relatively larger for the mediolateral dimensions of the inlet and midplane (but not the outlet - possibly due 
to allometric scaling; Table 2.5) as well as the dorsoventral diameters of all three pelvic planes (Table 2.7).

Birth Canal Shape: Males and females have significantly different birth canal shapes (Tables 2.10 - 2.12; Figures 2.10a and 2.10b), but there is considerable overlap between the sexes (Figure 2.10a; Table 2.14) such that $38 \%$ of females fall within the male distribution for birth canal shape. Females exhibit elongation of their pubes, more inferiorly-projecting superior pubic rami, and rostrocaudally shorter sacra (Figures 2.10a and 2.10b). Males exhibit rostro-caudally expanded sacra and longer pubic symphyses (Figure 2.10b).

\section{Pan troglodytes}

Birth Canal Size: In P. troglodytes, females are relatively (but not absolutely) larger than males for birth canal centroid size and the circumferences of all three pelvic planes (Table 2.6). Females achieve these large birth canals by having relatively larger transverse and dorsoventral diameters of the pelvic inlet, midplane, and outlet (Table 2.7).

Birth Canal Shape: Overall birth canal shape is significantly dimorphic in $P$. troglodytes (Tables 2.10-2.12; Figures 2.11a and 2.11b), but there is considerable overlap between the sexes (Figure 2.11a; Table 2.14), such that $79 \%$ of females fall within the male distribution for birth canal shape. Females have longer and more coronally-oriented pubes, and laterally flaring caudal ischia. Males have rostro-caudally longer sacra, pubic symphyses, and ischia (Figures 2.11a and 2.11b).

\section{Alouatta seniculus}


Birth Canal Size: In A. seniculus, there are no differences between males and females for relative (controlling for S1 body breadth) birth canal centroid size or circumferences of the inlet, midplane, or outlet (Table 2.6). There are also no statistical differences between sexes for the relative (controlling for S1 body breadth) mediolateral or dorsoventral diameters of any birth canal plane (Table 2.7).

Birth Canal Shape: Dimorphism in birth canal form is less pronounced in $A$. seniculus compared to other taxa (Tables 2.10-2.12; Figures 2.12a and 2.12b). There is considerable overlap between the sexes for birth canal shape (Table 2.14) such that $67 \%$ of females fall within the male distribution. Differences in the overall shape of the birth canal between males and females are insignificant (Tables 2.10 and 2.12). However, this species does exhibit a few localized sex differences in birth canal shape. Sexes significantly separate along PC2 (which encompasses $11 \%$ of shape variation in this species; Table 2.11), and males have relatively longer sacra and pubic symphyses (Figures 2.12a and 2.12b), and females have a slight anteroposterior expansion of the outlet.

\section{DISCUSSION AND CONCLUSIONS}

The results of this study support the hypothesis that birth canal dimorphism in nonhuman anthropoids is not due solely to large cephalopelvic proportions (Schultz, 1949; Steudel, 1981a; Tague, 1991; 2005), because size and shape of the birth canal are dimorphic in species with both large and small cephalopelvic proportions. These results are consistent with the presence of dimorphism in many species of mammals, regardless 
of neonate size (Gardner, 1936; Dunmire, 1955; Crelin and Newton, 1969; Chapman et al., 1994; Tague, 2003; Berdnikovs et al., 2007; Schutz et al., 2009a; Schutz et al., 2009b), (Tague, 2003; Schutz et al., 2009a; Schutz et al., 2009b).

The specific aspects contributing to this dimorphism are less clear. It has been hypothesized that dimorphism in relative sacral breadth is due to large cephalopelvic proportions (Moffett et al., 2013). If dimorphism in relative sacral breadth is only found among species with large cephalopelvic proportions, dimorphism in relative sacral breadth could be responsible for the increased magnitude of dimorphism in the birth canal in species with large cephalopelvic proportions. Moffett et al.'s (2013) hypothesis is further examined in Chapter 3. In contrast to sacral breadth, all species in this study are dimorphic in relative pubis length regardless of cephalopelvic proportions (except for A. seniculus), and this result is consistent with previous research which have found dimorphism in relative pubis length to be unrelated to cephalopelvic proportions (Steudel, 1981a). It is unlikely that dimorphism in pubis length accounts for the greater magnitude of dimorphism in the birth canals of species with large cephalopelvic proportions.

Previous authors have found that birth canal dimorphism is a correlate of dimorphism in overall body size, such that in species with high levels of body size dimorphism, females exhibit exceptionally large birth canals compared to males (Schultz, 1949; Leutenegger, 1974; Tague, 2005). The results of the present study partially support this hypothesis (Table 5.9). Among species with high levels of body size dimorphism and large cephalopelvic proportions (Nasalis larvatus, Papio anubis), the magnitude of dimorphism in birth canal centroid size (controlling for body size) is 
higher than any other taxa (dimorphism indices 130 and 121, respectively). In contrast, Gorilla gorilla (which exhibits large body size dimorphism and small cephalopelvic proportions) is minimally dimorphic in relative birth canal centroid size (dimorphism index of 103, the second lowest of all taxa). The combination of large cephalopelvic proportions and high levels of body size dimorphism (rather than only high levels of body size dimorphism) produces the highest magnitudes of dimorphism in relative birth canal size. In fact, Nasalis larvatus and Papio anubis have the least overlap in birth canal size, whereas most other taxa have a higher degree of overlap in birth canal size (see Appendix A). This means that differences in birth canal size may not be observable at small sample sizes in many taxa. In fossils for which we have more than one pelvis, it may be impossible to tell whether there is dimorphism in birth canal size due to small sample size.

Similar to dimorphism in birth canal size, dimorphism in birth canal shape (measured as overlap between males and females in shape space; Table 2.14) is greatest among species with both large cephalopelvic proportions and high body size dimorphism (Nasalis larvatus, Papio anubis) and lowest in species with small cephalopelvic proportions and low to intermediate body size dimorphism (Alouatta seniculus, Pan troglodytes). It should be noted that species that give birth to relatively large neonates but are less dimorphic in body size (i.e., Hylobates lar and Macaca mulatta) tend to have lower levels of dimorphism in birth canal size, more similar to what is seen in taxa with small cephalopelvic proportions (Table 5.9).

In contrast to other taxa, humans have low levels of body size dimorphism but a high magnitude of dimorphism in birth canal size and shape. Humans have the third 
highest magnitude of dimorphism in relative birth canal centroid size and relative inlet circumference (Table 2.10) and the highest magnitude of dimorphism in relative circumferences of the midplane and outlet despite exhibiting very low body size dimorphism. Furthermore, the shape of the birth canal is highly dimorphic in humans, and few females (10\%) fall within the male shape distribution (Table 2.14). Conversely, in H. lar (an ape that also has large cephalopelvic proportions and limited body size dimorphism) there is a low dimorphism index in relative birth canal centroid size and the relative circumferences of the inlet, midplane, and outlet, and there is a larger degree of overlap (33\%) between males and females for birth canal form in H. lar compared to humans. When compared with Saimiri sciureus (a species with even higher cephalopelvic proportions and body size dimorphism levels than humans), H. sapiens has a greater magnitude of dimorphism in birth canal centroid size and circumferences of the midplane and outlet (Table 2.10). Further, there is less shape overlap between the sexes in H. sapiens than S. sciureus (Table 2.14). The degree of pelvic dimorphism in humans cannot be accounted for based only on our large cephalopelvic proportions otherwise it would be expected that $H$. lar and S. sciureus would show a similar magnitude of dimorphism in birth canal size and shape as humans.

Why do humans have birth canals that are more dimorphic than other monomorphic (for body size) taxa with large cephalopelvic proportions? The high degree of dimorphism seen in the human pelvis may be related to the fact that in bipeds there is actually some risk associated with having a wider pelvis, possibly including greater frequency of knee injuries due to a high valgus angle, weakness of pelvic floor muscles and reduced support of pelvic viscera due to a greater distance between the 
ischia (Abitbol, 1988), or reduced bipedal locomotor efficiency due to an increased distance between the sacroiliac joints and acetabulae (Washburn, 1950, 1960; Pinkerton, 1973; Zihlman, 1978; Ruff, 1995; Lovejoy, 2005; contra Warrener et al., 2015).

Finally, in anthropoids, females are shown to have larger birth canal centroid sizes than males controlling for body size (all taxa except A. seniculus and G. gorilla). It is possible that females achieve these bigger birth canals by increasing the size of the entire pelvis, not just the parts of the pelvis which directly participate in the birth canal. Females also have mediolaterally wider inlets (all taxa except $A$. seniculus), midplanes (all taxa except A. seniculus, M. mulatta, and P. anubis), and outlets (all taxa except $A$. seniculus, P. anubis, and G. gorilla), and it is therefore likely that many aspects of the non-obstetric pelvis, or those parts which do not directly participate in the birth canal (including biacetabular breadth, ischial length and ischial tuberosity breadth, and inferior iliac height) are also dimorphic across primates. The hypothesis that dimorphism in the overall size and mediolateral dimensions of the birth canal correspond to dimorphism in parts of the pelvis which do not directly participate in the birth canal is tested in Chapter 4 . 
Table 2.1: Numbers of individuals, body mass dimorphism, and cephalopelvic proportions

\begin{tabular}{|c|c|c|c|c|}
\hline \multirow[t]{2}{*}{ Species } & \multicolumn{2}{|c|}{$\mathbf{N}$} & \multirow{2}{*}{$\begin{array}{c}\text { Body mass } \\
\text { dimorphism } \\
\text { index* } \\
(\mathrm{F} / \mathrm{M})\end{array}$} & \multirow{2}{*}{$\begin{array}{c}\text { Cephalopelvic } \\
\text { index }^{\dagger}\end{array}$} \\
\hline & $\mathbf{F}$ & $\mathbf{M}$ & & \\
\hline \multicolumn{5}{|c|}{ Large Cephalopelvic proportions } \\
\hline Homo sapiens & 73 & 83 & $1.14^{\S}$ & $1.02^{\ddagger}$ \\
\hline Hylobates lar & 55 & 57 & 1.11 & 0.94 \\
\hline Macaca mulatta & 106 & 81 & 1.24 & 1.00 \\
\hline Nasalis larvatus & 16 & 17 & 2.08 & 0.95 \\
\hline Papio anubis & 5 & 16 & 1.81 & 0.96 \\
\hline Saimiri sciureus & 75 & 78 & 1.46 & 1.21 \\
\hline \multicolumn{5}{|c|}{ Small Cephalopelvic Proportions } \\
\hline Pan troglodytes & 77 & 37 & 1.30 & 0.72 \\
\hline Gorilla gorilla & 50 & 56 & 2.38 & 0.64 \\
\hline Alouatta seniculus & 18 & 20 & 1.48 & 0.81 \\
\hline
\end{tabular}

* Values for body size dimorphism based on body mass values from Smith and Jungers (1997).

${ }^{\dagger}$ Calculated as (neonate head breadth/maternal pelvic inlet transverse diameter).Values for neonate head and maternal inlet from Schultz (1949).

${ }^{\S}$ Worldwide average body size dimorphism for humans (Smith and Jungers, 1997).

$\$$ The sagittal diameter of the neonate head and transverse breadth of the maternal inlet were used to build this ratio because this is how the human neonate modally engages the inlet.

Sample sizes differ between lumbar and sacral vertebrae because not all specimens included retained both a measurable sacrum and caudal-most lumbar vertebra. 
Table 2.2: Birth canal Landmarks

\section{Bilateral Landmarks}

\section{Description}

(1) SI joint at arcuate line*

Point on ilium where arcuate line intersects the sacroiliac joint

(2) Lateral arcuate line*

Lateral-most point of arcuate on articulated pelvis

(3) Pubis at arcuate line*

Arcuate at right angle to anterior acetabular rim

(4) Ischial spine*

Ischial spine on posterior ischium

(5) Posterior ischial tuberosity*

Posterior-most point on the ischial tuberosity

\section{Midline Landmarks}

Description

(6) Inferior pubic symphysis*

Inferior-most point on symphysis at the midline

(7) Superior pubic symphysis*

Superior-most point on symphysis at the midline

(8) Ventral S1body

(9) Inferior S1 body*

Ventral-most point on the first vertebral body at midline

Inferior S1 vertebral body at midline

(10) Inferior sacrum*

Inferior-most point on sacrum at midline

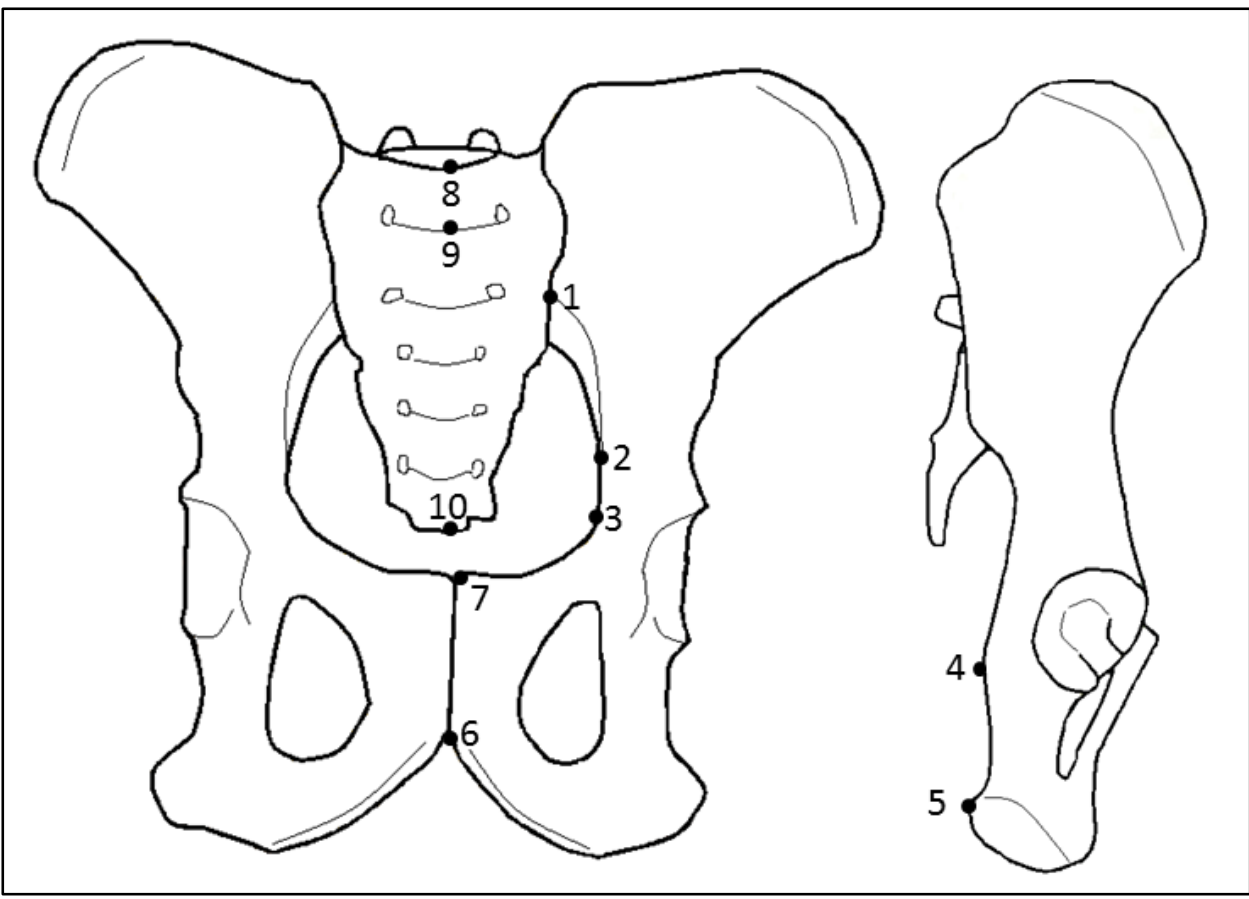

Figure 2.1: Landmarks collected on the birth canal. (1) Sacroiliac joint at arcuate line; (2) Lateral arcuate line (3) Pubis at arcuate line; (4) Ischial spine; (5) posterior ischial tuberosity; (6) inferior pubic symphysis; (7) superior pubic symphysis; (8) ventral S1 body; (9) inferior S1 body; (10) inferior sacrum. 


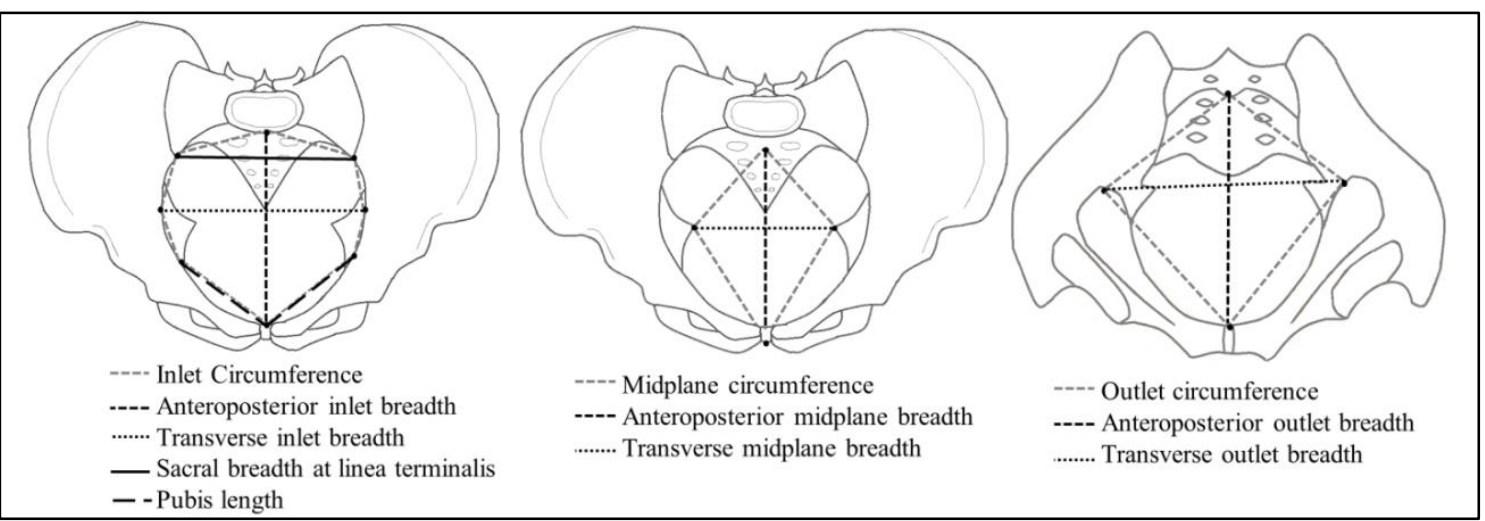

Figure 2.2: Linear distances in the birth canal secondarily calculated from landmark data. For the inlet, circumference is calculated as the sum of the bilateral linear distances between the anterior S1 body and sacroiliac joint at arcuate line, sacroiliac joint at arcuate line and lateral arcuate line and arcuate line at acetabulum, and arcuate line at acetabulum and superior pubic symphysis. Midplane and outlet circumferences are calculated as the bilateral sum of [distance between inferior sacrum and ischial spine + distance between ischial spine and inferior pubic symphysis] and [distance between inferior sacrum and posterior ischial tuberosity + distance between posterior ischial tuberosity and inferior pubic symphysis] respectively, following Tague (1991). Dorsoventral and transverse diameters of the inlet and midplane are calculated following Tague (1991) secondarily from landmark data.

\begin{tabular}{|c|c|}
\hline \multicolumn{2}{|c|}{ Table 2.3: Birth canal dimension calculations } \\
\hline Dimension & Calculation \\
\hline Inlet Circumference & $\begin{array}{l}\text { Sum of linear distances between landmark } 8 \text { and } 1 ; 1 \text { and } 2 ; 2 \text { and } 3 ; 3 \text { and } 7 \\
\text { on right and left sides }\end{array}$ \\
\hline Midplane Circumference & $\begin{array}{l}\text { Sum of linear distances between landmark } 9 \text { and } 4 \text {, and } 4 \text { and } 6 \text { on right and } \\
\text { left sides }\end{array}$ \\
\hline Outlet Circumference & $\begin{array}{l}\text { Sum of linear distances between landmark } 10 \text { and } 5 \text {, and } 5 \text { and } 6 \text { on right and } \\
\text { left sides }\end{array}$ \\
\hline Mediolateral Inlet Diameter & Linear distance between bilateral landmarks 2 \\
\hline Mediolateral Midplane Diameter & Linear distance between bilateral landmarks 4 \\
\hline Mediolateral Outlet Diameter & Linear distance between bilateral landmarks 5 \\
\hline Dorsoventral Inlet Diameter & Linear distance between landmarks 7 and 8 \\
\hline Dorsoventral Midplane Diameter & Linear distance between landmarks 6 and 9 \\
\hline Dorsoventral Outlet Diameter & Linear distance between landmarks 6 and 10 \\
\hline
\end{tabular}


Table 2.4: Reduced major axis regressions of natural logged birth canal size against S1 body breadth

\begin{tabular}{|c|c|c|c|c|}
\hline & \multicolumn{4}{|c|}{ x S1 Body Breadth } \\
\hline & $\mathbf{r}^{2}$ & $\begin{array}{c}\text { Slope } \\
\text { Anthropoids } \\
\text { (C.I) }\end{array}$ & $\begin{array}{l}\text { Slope } \\
\text { Apes } \\
\text { (C.I) } \\
\end{array}$ & $\begin{array}{c}\text { Slope } \\
\text { Monkeys } \\
\text { (C.I) }\end{array}$ \\
\hline $\begin{array}{l}\text { Birth Canal } \\
\text { Centroid Size }\end{array}$ & 0.88 & $\begin{array}{c}1.02 * \\
(0.99-1.06)\end{array}$ & $\begin{array}{c}1.02 * \\
(0.97-1.05)\end{array}$ & $\begin{array}{c}0.94 * \\
(0.88-1.02)\end{array}$ \\
\hline $\begin{array}{l}\text { Inlet } \\
\text { Circumference }\end{array}$ & 0.91 & $\begin{array}{c}1.02 * \\
(1.00-1.06)\end{array}$ & $\begin{array}{c}0.99 * \\
(0.95-1.02)\end{array}$ & $\begin{array}{c}0.95^{*} \\
(0.89-1.01)\end{array}$ \\
\hline $\begin{array}{l}\text { Midplane } \\
\text { Circumference }\end{array}$ & 0.84 & $\begin{array}{c}1.05 \\
(1.01-1.09)\end{array}$ & $\begin{array}{c}1.00 * \\
(0.97-1.04)\end{array}$ & $\begin{array}{c}0.98^{*} \\
(0.90-1.07)\end{array}$ \\
\hline $\begin{array}{l}\text { Outlet } \\
\text { Circumference }\end{array}$ & 0.88 & $\begin{array}{c}0.99 * \\
(0.95-1.20)\end{array}$ & $\begin{array}{c}1.08 \\
(1.01-1.11)\end{array}$ & $\begin{array}{c}0.95 * \\
(0.90-1.02)\end{array}$ \\
\hline
\end{tabular}

Regressions were performed on male specimens only.

*Denotes isometry

\begin{tabular}{|c|c|c|c|c|}
\hline & & x S1 & ody Breadth & \\
\hline & $\mathbf{r}^{2}$ & $\begin{array}{c}\text { Slope } \\
\text { Anthropoids } \\
\text { (C.I) }\end{array}$ & $\begin{array}{l}\text { Slope } \\
\text { Apes } \\
\text { (C.I) }\end{array}$ & $\begin{array}{c}\text { Slope } \\
\text { Monkeys } \\
\text { (C.I) }\end{array}$ \\
\hline $\begin{array}{l}\text { Mediolateral } \\
\text { Inlet }\end{array}$ & 0.91 & $\begin{array}{c}1.09 \\
(1.06-1.12)\end{array}$ & $\begin{array}{c}1.00 * \\
(0.96-1.04)\end{array}$ & $\begin{array}{c}0.93 * \\
(0.87-1.00)\end{array}$ \\
\hline $\begin{array}{l}\text { Mediolateral } \\
\text { Midplane }\end{array}$ & 0.87 & $\begin{array}{c}1.08 \\
(1.05-1.12)\end{array}$ & $\begin{array}{c}0.94 \\
(0.89-0.98)\end{array}$ & $\begin{array}{c}1.05 \\
(0.97-1.15)\end{array}$ \\
\hline $\begin{array}{l}\text { Mediolateral } \\
\text { Outlet }\end{array}$ & 0.85 & $\begin{array}{c}1.16 \\
(1.12-1.21) \\
\end{array}$ & $\begin{array}{c}1.29 \\
(1.23-1.35) \\
\end{array}$ & $\begin{array}{c}0.82 \\
(0.77-0.92) \\
\end{array}$ \\
\hline $\begin{array}{l}\text { Dorsoventral } \\
\text { Inlet }\end{array}$ & 0.81 & $\begin{array}{c}1.09 \\
(1.04-1.13)\end{array}$ & $\begin{array}{c}0.98^{*} \\
(0.94-1.02)\end{array}$ & $\begin{array}{c}1.03 * \\
(0.98-1.09)\end{array}$ \\
\hline $\begin{array}{l}\text { Dorsoventral } \\
\text { Midplane }\end{array}$ & 0.83 & $\begin{array}{c}1.11 \\
(1.07-1.15)\end{array}$ & $\begin{array}{c}1.00 * \\
(0.96-1.03)\end{array}$ & $\begin{array}{c}1.18 \\
(1.09-1.26)\end{array}$ \\
\hline $\begin{array}{l}\text { Dorsoventral } \\
\text { Outlet }\end{array}$ & 0.85 & $\begin{array}{c}1.07 \\
(1.03-1.11) \\
\end{array}$ & $\begin{array}{c}0.94 \\
(0.90-0.99) \\
\end{array}$ & $\begin{array}{c}1.24 \\
(1.14-1.32) \\
\end{array}$ \\
\hline
\end{tabular}

Bold denotes isometry.

*Regressions were performed on male specimens only. 


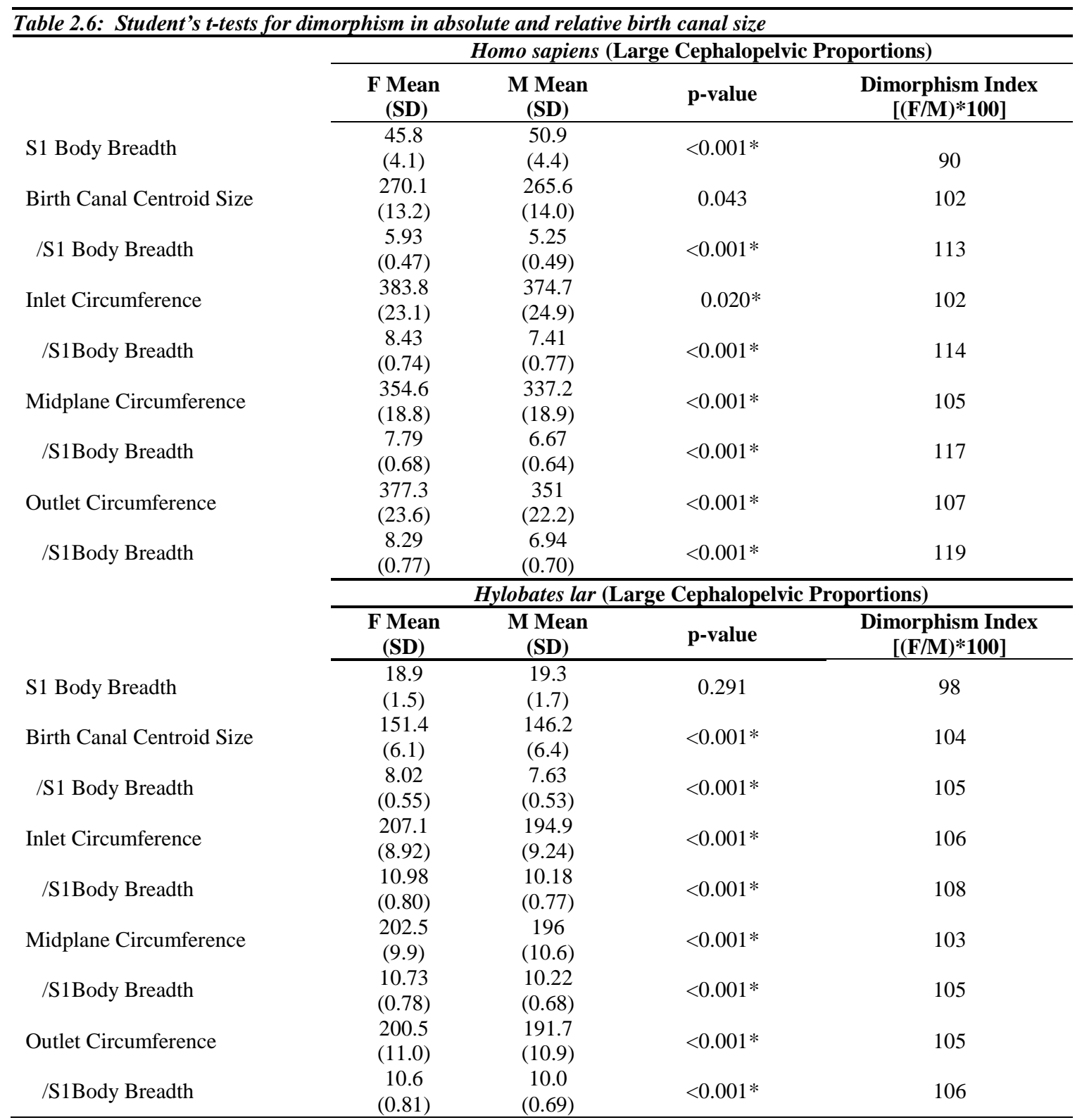

Absolute measurements are in millimeters.

*Indicates significance at $\alpha=0.035$. 
Table 2.6 (cot'd): Student's t-tests for dimorphism in absolute and relative birth canal size

S1 Body Breadth

Birth Canal Centroid Size

/S1 Body Breadth

Inlet Circumference

/S1Body Breadth

Midplane Circumference

/S1Body Breadth

Outlet Circumference

/S1Body Breadth

S1 Body Breadth

Birth Canal Centroid Size

/S1 Body Breadth

Inlet Circumference

/S1Body Breadth

Midplane Circumference

/S1Body Breadth

Outlet Circumference

/S1Body Breadth

\begin{tabular}{c}
\hline F Mean \\
(SD)
\end{tabular}

Macaca mulatta (Large Cephalopelvic Proportions)

20.9

(1.2)

142.9

(6.2)

6.85

(0.45)

197.0

(11.2)

9.46

(0.74)

195.0

(12.4)

9.34

(0.75)

227.0

(13.4)

10.9

(0.85)

\begin{tabular}{r} 
\\
\hline F M \\
(SD) \\
\hline 22.7 \\
$(1.0)$ \\
156.1 \\
$(10.8)$
\end{tabular}

Macaca mulatta
M Mean

(SD)

23.7

(1.6)

154.3

(7.0)

6.53

(0.42)

202.3

(11.5)

8.56

(0.66)

206.0

(10.3)

8.72

(0.61)

226.0

(11.1)

9.56

(0.63)

Nasalis larvatus (Large Cephalopelvic Proportions) p-value

Dimorphism Index $[(\mathbf{F} / \mathbf{M}) * 100]$

$<0.001 * \quad 88$

$<0.001 * \quad 93$

$<0.001 * \quad 105$

$\begin{array}{ll}<0.001 * & 97\end{array}$

$<0.001 * \quad 111$

$<0.001 * \quad 95$

$\begin{array}{ll}<0.001 * & 107\end{array}$

$\begin{array}{ll}0.550 & 100\end{array}$

$<0.001 * \quad 114$
(10.8)

6.91

(0.64)

200.4

(11.0)

8.84

(0.37)

161.8

(14.6)

7.13

(0.50)

201.6

(15.2)

8.89

$(0.54)$

(SD)

29.0

(3.2)

153.1

(13.5)

5.33

$(0.75)$

208.7

(13.5)

7.25

$(0.81)$

180.5

(16.3)

6.27

(0.78)

231.3

(12.9)

8.03

$(0.81)$ $[(\mathbf{F} / \mathbf{M}) * 100]$

Absolute measurements are in millimeters.

*Indicates significance at $\alpha=0.035$. 
Table 2.6 (cot'd): Student's t-tests for dimorphism in absolute and relative birth canal size

\begin{tabular}{|c|c|c|c|c|}
\hline & \multicolumn{4}{|c|}{ Papio anubis (Large Cephalopelvic Proportions) } \\
\hline & $\begin{array}{l}\text { F Mean } \\
\text { (SD) }\end{array}$ & $\begin{array}{l}\text { M Mean } \\
\text { (SD) }\end{array}$ & p-value & $\begin{array}{c}\text { Dimorphism Index } \\
{[(\mathbf{F} / \mathbf{M}) * 100]}\end{array}$ \\
\hline S1 Body Breadth & $\begin{array}{l}27.1 \\
(2.2)\end{array}$ & $\begin{array}{l}32.4 \\
(1.9)\end{array}$ & $<0.001 *$ & 84 \\
\hline Birth Canal Centroid Size & $\begin{array}{l}199.6 \\
(12.3)\end{array}$ & $\begin{array}{l}197.4 \\
(12.5)\end{array}$ & 0.739 & 101 \\
\hline /S1 Body Breadth & $\begin{array}{c}7.41 \\
(0.71)\end{array}$ & $\begin{array}{c}6.12 \\
(0.54)\end{array}$ & $<0.001 *$ & 121 \\
\hline Inlet Circumference & $\begin{array}{l}252.3 \\
(17.9)\end{array}$ & $\begin{array}{l}269.4 \\
(15.4)\end{array}$ & 0.105 & 94 \\
\hline /S1Body Breadth & $\begin{array}{c}9.33 \\
(0.34)\end{array}$ & $\begin{array}{c}8.33 \\
(0.37)\end{array}$ & $<0.001 *$ & 112 \\
\hline Midplane Circumference & $\begin{array}{l}243.7 \\
(10.9)\end{array}$ & $\begin{array}{l}259.4 \\
(13.8)\end{array}$ & $0.028 *$ & 94 \\
\hline /S1Body Breadth & $\begin{array}{c}9.03 \\
(0.42)\end{array}$ & $\begin{array}{c}8.03 \\
(0.41)\end{array}$ & $0.003 *$ & 112 \\
\hline Outlet Circumference & $\begin{array}{l}287.5 \\
(15.2)\end{array}$ & $\begin{array}{l}300.1 \\
(20.7)\end{array}$ & 0.176 & 96 \\
\hline \multirow[t]{3}{*}{ /S1Body Breadth } & $\begin{array}{l}10.65 \\
(0.66)\end{array}$ & $\begin{array}{c}9.28 \\
(0.57)\end{array}$ & $0.006^{*}$ & 115 \\
\hline & \multicolumn{4}{|c|}{ Saimiri sciureus (Large Cephalopelvic Proportions) } \\
\hline & $\begin{array}{c}\text { F Mean } \\
\text { (SD) }\end{array}$ & $\begin{array}{l}\text { M Mean } \\
\text { (SD) }\end{array}$ & p-value & $\begin{array}{c}\text { Dimorphism Index } \\
{[(\mathbf{F} / \mathbf{M}) * 100]}\end{array}$ \\
\hline S1 Body Breadth & $\begin{array}{l}10.0 \\
(0.6)\end{array}$ & $\begin{array}{l}10.4 \\
(0.7)\end{array}$ & $<0.001 *$ & 96 \\
\hline Birth Canal Centroid Size & $\begin{array}{l}65.7 \\
(3.1)\end{array}$ & $\begin{array}{l}62.3 \\
(3.7)\end{array}$ & $<0.001 *$ & 105 \\
\hline /S1 Body Breadth & $\begin{array}{c}6.62 \\
(0.42)\end{array}$ & $\begin{array}{c}6.01 \\
(0.37)\end{array}$ & $<0.001 *$ & 110 \\
\hline Inlet Circumference & $\begin{array}{l}94.9 \\
(4.3)\end{array}$ & $\begin{array}{l}82.9 \\
(5.8)\end{array}$ & $<0.001 *$ & 114 \\
\hline /S1Body Breadth & $\begin{array}{c}9.56 \\
(0.66)\end{array}$ & $\begin{array}{c}7.99 \\
(0.59)\end{array}$ & $<0.001 *$ & 120 \\
\hline Midplane Circumference & $\begin{array}{l}85.6 \\
(6.6)\end{array}$ & $\begin{array}{l}77.8 \\
(6.9)\end{array}$ & $<0.001 *$ & 110 \\
\hline /S1Body Breadth & $\begin{array}{c}8.61 \\
(0.70)\end{array}$ & $\begin{array}{c}7.49 \\
(0.60)\end{array}$ & $<0.001 *$ & 115 \\
\hline Outlet Circumference & $\begin{array}{l}96.3 \\
(5.9)\end{array}$ & $\begin{array}{l}90.4 \\
(7.0)\end{array}$ & $<0.001 *$ & 107 \\
\hline /S1Body Breadth & $\begin{array}{c}9.70 \\
(0.79)\end{array}$ & $\begin{array}{c}8.72 \\
(0.70)\end{array}$ & $<0.001 *$ & 111 \\
\hline
\end{tabular}
Papio anubis (Large Cephalopelvic Proportions)

Absolute measurements are in millimeters.

*Indicates significance at $\alpha=0.035$. 
Table 2.6 (cot'd): Student's t-tests for dimorphism in absolute and relative birth canal size

S1 Body Breadth

Birth Canal Centroid Size

/S1 Body Breadth

Inlet Circumference

/S1Body Breadth

Midplane Circumference

/S1Body Breadth

Outlet Circumference

/S1Body Breadth

S1 Body Breadth

Birth Canal Centroid Size

/S1 Body Breadth

Inlet Circumference

/S1Body Breadth

Midplane Circumference

/S1Body Breadth

Outlet Circumference

/S1Body Breadth

\begin{tabular}{|c|c|c|c|}
\hline \multicolumn{4}{|c|}{ Gorilla gorilla (Small Cephalopelvic Proportions) } \\
\hline $\begin{array}{l}\text { F Mean } \\
\text { (SD) }\end{array}$ & $\begin{array}{c}\text { M Mean } \\
\text { (SD) }\end{array}$ & p-value & $\begin{array}{c}\text { Dimorphism Index } \\
{[(\mathbf{F} / \mathbf{M}) * 100]}\end{array}$ \\
\hline $\begin{array}{l}41.3 \\
(3.9)\end{array}$ & $\begin{array}{l}48.8 \\
(5.3)\end{array}$ & $<0.001 *$ & 85 \\
\hline $\begin{array}{l}333.9 \\
(21.5)\end{array}$ & $\begin{array}{l}381.9 \\
(18.3)\end{array}$ & $<0.001 *$ & 87 \\
\hline $\begin{array}{c}8.12 \\
(0.64)\end{array}$ & $\begin{array}{c}7.89 \\
(0.69)\end{array}$ & 0.072 & 103 \\
\hline $\begin{array}{l}447.9 \\
(33.3)\end{array}$ & $\begin{array}{l}494.7 \\
(28.1)\end{array}$ & $<0.001 *$ & 91 \\
\hline $\begin{array}{l}10.89 \\
(0.92)\end{array}$ & $\begin{array}{l}10.21 \\
(0.90)\end{array}$ & $<0.001 *$ & 107 \\
\hline $\begin{array}{l}451.7 \\
(31.8)\end{array}$ & $\begin{array}{l}500.2 \\
(29.7)\end{array}$ & $<0.001 *$ & 90 \\
\hline $\begin{array}{l}10.99 \\
(0.89)\end{array}$ & $\begin{array}{l}10.32 \\
(0.92)\end{array}$ & $<0.001 *$ & 106 \\
\hline $\begin{array}{l}461.1 \\
(38.3)\end{array}$ & $\begin{array}{l}521.1 \\
(37.0)\end{array}$ & $<0.001 *$ & 88 \\
\hline $\begin{array}{l}11.23 \\
(1.21) \\
\end{array}$ & $\begin{array}{l}10.79 \\
(1.32) \\
\end{array}$ & 0.073 & 104 \\
\hline \multicolumn{4}{|c|}{ Pan troglodytes (Small Cephalopelvic Proportions) } \\
\hline $\begin{array}{l}\text { F Mean } \\
\text { (SD) }\end{array}$ & $\begin{array}{c}\begin{array}{c}\text { M Mean } \\
\text { (SD) }\end{array} \\
\end{array}$ & p-value & $\begin{array}{c}\text { Dimorphism Index } \\
{[(\mathbf{F} / \mathbf{M}) * 100]}\end{array}$ \\
\hline $\begin{array}{l}34.5 \\
(3.3)\end{array}$ & $\begin{array}{l}36.1 \\
(3.0)\end{array}$ & $0.013 *$ & 96 \\
\hline $\begin{array}{l}292.1 \\
(14.6)\end{array}$ & $\begin{array}{l}287.6 \\
(11.5)\end{array}$ & 0.103 & 102 \\
\hline $\begin{array}{c}8.53 \\
(0.77)\end{array}$ & $\begin{array}{c}8.01 \\
(0.76)\end{array}$ & $0.001 *$ & 106 \\
\hline $\begin{array}{l}380.3 \\
(22.7)\end{array}$ & $\begin{array}{l}365.4 \\
(17.9)\end{array}$ & $<0.001 *$ & 104 \\
\hline $\begin{array}{l}11.10 \\
(1.06)\end{array}$ & $\begin{array}{l}10.17 \\
(1.05)\end{array}$ & $<0.001 *$ & 109 \\
\hline $\begin{array}{l}399.4 \\
(23.3)\end{array}$ & $\begin{array}{l}389.7 \\
(17.2)\end{array}$ & $0.016^{*}$ & 102 \\
\hline $\begin{array}{l}11.65 \\
(0.96)\end{array}$ & $\begin{array}{l}10.85 \\
(1.05)\end{array}$ & $<0.001 *$ & 107 \\
\hline $\begin{array}{l}413.0 \\
(28.2)\end{array}$ & $\begin{array}{l}402.7 \\
(25.0)\end{array}$ & 0.066 & 103 \\
\hline $\begin{array}{l}12.06 \\
(1.20)\end{array}$ & $\begin{array}{r}11.27 \\
(1.11)\end{array}$ & $<0.001 *$ & 107 \\
\hline
\end{tabular}

Absolute measurements are in millimeters.

*Indicates significance at $\alpha=0.035$. 
Table 2.6 (cot'd): Student's t-tests for dimorphism in absolute and relative birth canal size

\begin{tabular}{|c|c|c|c|c|}
\hline & \multicolumn{4}{|c|}{ Alouatta seniculus (Small Cephalopelvic Proportions) } \\
\hline & $\begin{array}{l}\text { F Mean } \\
\text { (SD) }\end{array}$ & $\begin{array}{l}\text { M Mean } \\
\text { (SD) }\end{array}$ & p-value & $\begin{array}{c}\text { Dimorphism Index } \\
{\left[(\mathbf{F} / \mathbf{M})^{* 100}\right]}\end{array}$ \\
\hline S1 Body Breadth & $\begin{array}{l}34.3 \\
(2.9)\end{array}$ & $\begin{array}{l}36.2 \\
(2.9)\end{array}$ & 0.044 & 95 \\
\hline Birth Canal Centroid Size & $\begin{array}{l}135.8 \\
(8.0)\end{array}$ & $\begin{array}{l}144.0 \\
(10.5)\end{array}$ & $0.015^{*}$ & 94 \\
\hline /S1 Body Breadth & $\begin{array}{c}3.97 \\
(0.24)\end{array}$ & $\begin{array}{c}3.98 \\
(0.18)\end{array}$ & 0.949 & 100 \\
\hline Inlet Circumference & $\begin{array}{l}191.7 \\
(11.8)\end{array}$ & $\begin{array}{l}202.6 \\
(14.9)\end{array}$ & 0.165 & 95 \\
\hline /S1Body Breadth & $\begin{array}{c}5.61 \\
(0.31)\end{array}$ & $\begin{array}{c}5.60 \\
(0.27)\end{array}$ & 0.988 & 100 \\
\hline Midplane Circumference & $\begin{array}{l}160.1 \\
(11.7)\end{array}$ & $\begin{array}{l}164.9 \\
(17.4)\end{array}$ & 0.325 & 97 \\
\hline /S1Body Breadth & $\begin{array}{c}4.68 \\
(0.27)\end{array}$ & $\begin{array}{c}4.55 \\
(0.39)\end{array}$ & 0.265 & 103 \\
\hline Outlet Circumference & $\begin{array}{l}202.7 \\
(13.3)\end{array}$ & $\begin{array}{l}213.7 \\
(16.6)\end{array}$ & $0.030 *$ & 95 \\
\hline /S1Body Breadth & $\begin{array}{c}5.93 \\
(0.40)\end{array}$ & $\begin{array}{c}5.90 \\
(0.29)\end{array}$ & 0.818 & 100 \\
\hline
\end{tabular}

Absolute measurements are in millimeters.

*Indicates significance at $\alpha=0.035$. 
Table 2.7: Student's t-tests for dimorphism in absolute and relative birth canal dimensions

\begin{tabular}{|c|c|c|c|c|c|c|c|c|}
\hline & \multicolumn{4}{|c|}{ Homo sapiens } & \multicolumn{4}{|c|}{ Hylobates lar } \\
\hline & $\begin{array}{c}\text { F } \\
\text { Mean }\end{array}$ & $\begin{array}{c}\text { M } \\
\text { Mean }\end{array}$ & p-value & $\begin{array}{c}\text { Dimorphism } \\
\text { Index } \\
{\left[(\mathbf{F} / \mathbf{M})^{* 100}\right]} \\
\end{array}$ & $\underset{\text { Mean }}{\mathbf{F}}$ & $\begin{array}{c}\text { M } \\
\text { Mean }\end{array}$ & p-value & $\begin{array}{c}\text { Dimorphism } \\
\text { Index } \\
{\left[(\mathbf{F} / \mathbf{M})^{* 100}\right]} \\
\end{array}$ \\
\hline \multirow{2}{*}{$\begin{array}{l}\text { Transverse Inlet Diameter } \\
\text { /S1Body Breadth }\end{array}$} & 125.1 & 122.6 & 0.108 & 102 & 57.3 & 53.5 & $<0.001 *$ & 107 \\
\hline & 2.75 & 2.42 & $<0.001 *$ & 114 & 3.04 & 2.79 & $<0.001 *$ & 109 \\
\hline \multirow{2}{*}{$\begin{array}{l}\text { Transverse Midplane } \\
\text { Diameter } \\
\text { /S1Body Breadth }\end{array}$} & 104.9 & 87.6 & $<0.001 *$ & 120 & 49.4 & 45.5 & $<0.001 *$ & 109 \\
\hline & 2.31 & 1.73 & $<0.001 *$ & 134 & 2.62 & 2.37 & $<0.001 *$ & 111 \\
\hline \multirow{2}{*}{$\begin{array}{l}\text { Transverse Outlet } \\
\text { Diameter } \\
\text { /S1Body Breadth }\end{array}$} & 134.7 & 120.9 & $<0.001 *$ & 111 & 54.9 & 50.1 & $<0.001 *$ & 110 \\
\hline & 2.96 & 2.39 & $<0.001 *$ & 124 & 2.91 & 2.61 & $<0.001 *$ & 111 \\
\hline \multirow{2}{*}{$\begin{array}{l}\text { Dorsoventral Inlet } \\
\text { Diameter } \\
\text { /S1Body Breadth }\end{array}$} & 114.3 & 108.2 & $<0.001 *$ & 106 & 79.0 & 75.7 & $<0.001 *$ & 104 \\
\hline & 2.51 & 2.14 & $<0.001^{*}$ & 117 & 4.19 & 3.95 & $<0.001 *$ & 106 \\
\hline \multirow{2}{*}{$\begin{array}{l}\text { Dorsoventral Midplane } \\
\text { Diameter } \\
\text { /S1Body Breadth }\end{array}$} & 128.6 & 127.0 & 0.289 & 101 & 83.4 & 81.2 & $0.019 *$ & 103 \\
\hline & 2.82 & 2.51 & $<0.001 *$ & 112 & 4.42 & 4.24 & $0.004 *$ & 104 \\
\hline \multirow{4}{*}{$\begin{array}{l}\text { Dorsoventral Outlet } \\
\text { Diameter } \\
\text { /S1Body Breadth }\end{array}$} & 121.8 & 113.7 & $<0.001 *$ & 107 & 69.8 & 67.3 & $0.005^{*}$ & 104 \\
\hline & 2.68 & 2.25 & $<0.001 *$ & 119 & 3.70 & 3.51 & $<0.001 *$ & 105 \\
\hline & \multicolumn{4}{|c|}{ Macaca mulatta } & \multicolumn{4}{|c|}{ Nasalis larvatus } \\
\hline & $\begin{array}{c}\mathbf{F} \\
\text { Mean }\end{array}$ & $\begin{array}{c}\text { M } \\
\text { Mean }\end{array}$ & p-value & $\begin{array}{c}\text { Dimorphism } \\
\text { Index } \\
{\left[(\mathbf{F} / \mathbf{M})^{* 100}\right]} \\
\end{array}$ & $\underset{\text { Mean }}{\mathbf{F}}$ & $\underset{\text { Mean }}{\text { M }}$ & p-value & $\begin{array}{c}\text { Dimorphism } \\
\text { Index } \\
{[(\mathbf{F} / \mathrm{M}) * 100]} \\
\end{array}$ \\
\hline \multirow{2}{*}{$\begin{array}{l}\text { Transverse Inlet Diameter } \\
\text { /S1Body Breadth }\end{array}$} & 50.2 & 52.1 & $0.003 *$ & 96 & 54.7 & 60.7 & $<0.001 *$ & 90 \\
\hline & 2.41 & 2.20 & $<0.001 *$ & 110 & 2.41 & 2.11 & $<0.001^{*}$ & 114 \\
\hline \multirow{2}{*}{$\begin{array}{l}\text { Transverse Midplane } \\
\text { Diameter } \\
\text { /S1Body Breadth }\end{array}$} & 43.3 & 47.2 & $<0.001 *$ & 92 & 43.8 & 40.2 & $0.034 *$ & 109 \\
\hline & 2.07 & 2.00 & 0.045 & 104 & 1.93 & 1.40 & $<0.001 *$ & 138 \\
\hline \multirow{2}{*}{$\begin{array}{l}\text { Transverse Outlet } \\
\text { Diameter } \\
\text { /S1Body Breadth }\end{array}$} & 51.6 & 51.5 & 0.863 & 100 & 42.0 & 37.1 & $0.008 *$ & 113 \\
\hline & 2.47 & 2.18 & $<0.001 *$ & 113 & 1.85 & 1.29 & $<0.001 *$ & 143 \\
\hline \multirow{2}{*}{$\begin{array}{l}\text { Dorsoventral Inlet } \\
\text { Diameter } \\
\text { /S1Body Breadth }\end{array}$} & 67.3 & 68.9 & $0.012 *$ & 98 & 70.9 & 71.1 & 0.929 & 100 \\
\hline & 3.23 & 2.91 & $<0.001 *$ & 111 & 3.12 & 2.47 & $<0.001 *$ & 126 \\
\hline \multirow{2}{*}{$\begin{array}{l}\text { Dorsoventral Midplane } \\
\text { Diameter } \\
\text { /S1Body Breadth }\end{array}$} & 77.3 & 86.5 & $<0.001 *$ & 89 & 74.8 & 88.7 & $<0.001 *$ & 84 \\
\hline & 3.71 & 3.66 & 0.244 & 101 & 3.30 & 3.08 & 0.039 & 107 \\
\hline \multirow{2}{*}{$\begin{array}{l}\text { Dorsoventral Outlet } \\
\text { Diameter } \\
\text { /S1Body Breadth }\end{array}$} & 73.8 & 79.9 & $<0.001 *$ & 92 & 67.9 & 80.2 & $<0.001 *$ & 85 \\
\hline & 3.54 & 3.38 & $<0.001 *$ & 105 & 2.99 & 2.79 & $0.030 *$ & 107 \\
\hline
\end{tabular}

*Indicates males and females exhibit statistical differences at $\alpha=0.035$. 
Table 2.7 (cot'd): Student's t-tests for dimorphism in absolute and relative birth canal dimensions

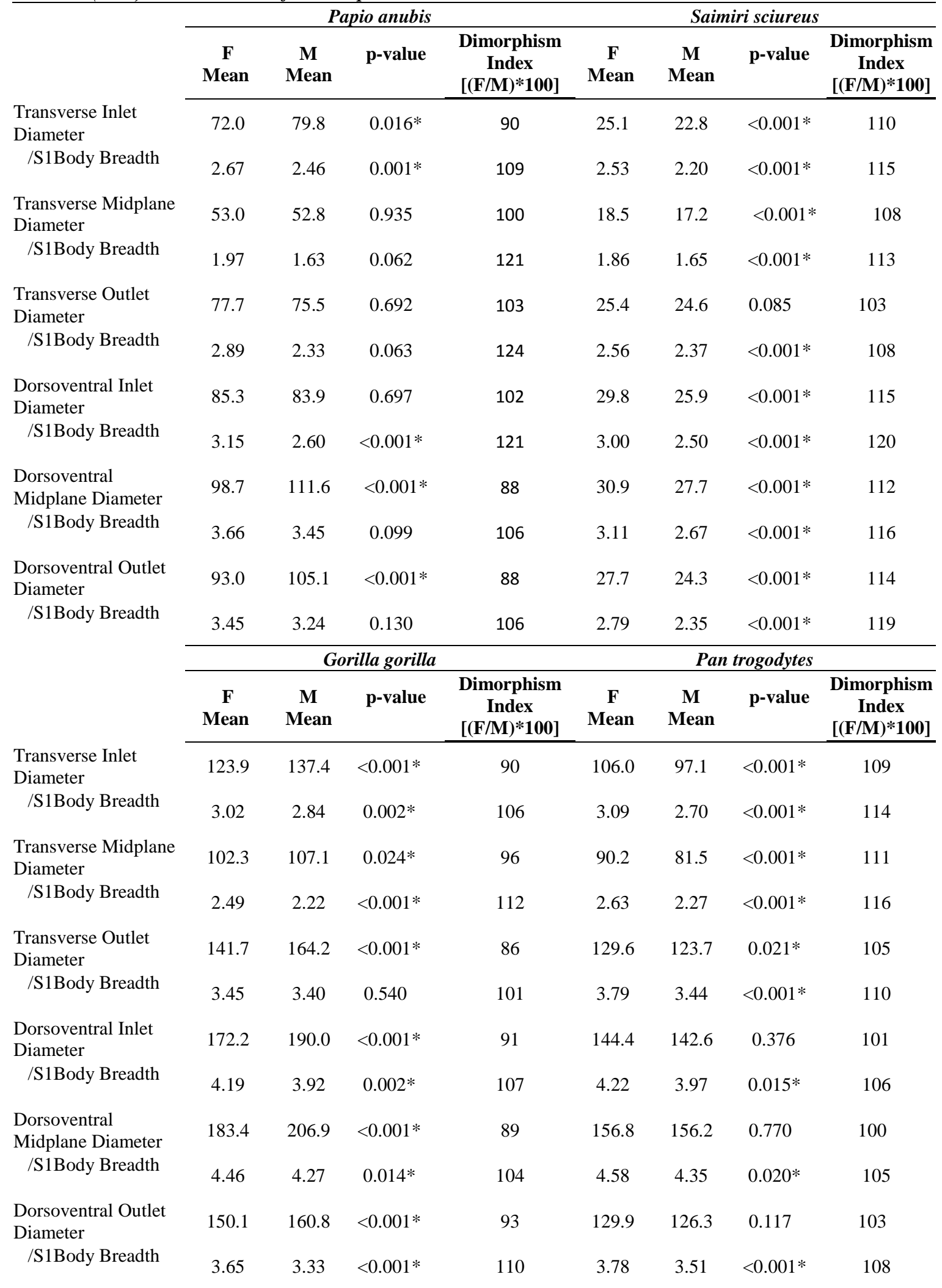

*Indicates males and females exhibit statistical differences at $\alpha=0.035$. 


\begin{tabular}{|c|c|c|c|c|}
\hline \multicolumn{5}{|c|}{$\begin{array}{l}\text { Table } 2.7 \text { (cot'd): Student's t-tests for dimorphism in absolute and relative } \\
\text { birth canal dimensions }\end{array}$} \\
\hline & \multicolumn{4}{|c|}{ Alouatta seniculus } \\
\hline & $\begin{array}{c}\mathbf{F} \\
\text { Mean }\end{array}$ & $\begin{array}{c}\text { M } \\
\text { Mean }\end{array}$ & p-value & $\begin{array}{c}\text { Dimorphism } \\
\text { Index } \\
{\left[(\mathbf{F} / \mathbf{M})^{* 100}\right]} \\
\end{array}$ \\
\hline Transverse Inlet Diameter & 48.9 & 51.2 & 0.175 & 96 \\
\hline /S1Body Breadth & 2.88 & 2.85 & 0.742 & 101 \\
\hline \multirow{2}{*}{$\begin{array}{l}\text { Transverse Midplane } \\
\text { Diameter } \\
\text { /S1Body Breadth }\end{array}$} & 42.0 & 42.7 & 0.674 & 98 \\
\hline & 2.48 & 2.37 & 0.210 & 105 \\
\hline \multirow{2}{*}{$\begin{array}{l}\text { Transverse Outlet } \\
\text { Diameter } \\
\text { /S1Body Breadth }\end{array}$} & 54.3 & 58.1 & 0.084 & 93 \\
\hline & 3.20 & 3.22 & 0.855 & 99 \\
\hline \multirow{2}{*}{$\begin{array}{l}\text { Dorsoventral Inlet } \\
\text { Diameter } \\
\text { /S1Body Breadth }\end{array}$} & 70.1 & 73.5 & 0.045 & 95 \\
\hline & 4.14 & 4.10 & 0.777 & 101 \\
\hline \multirow{2}{*}{$\begin{array}{l}\text { Dorsoventral Midplane } \\
\text { Diameter } \\
\text { /S1Body Breadth }\end{array}$} & 71.7 & 74.9 & 0.882 & 96 \\
\hline & 4.23 & 4.17 & 0.584 & 101 \\
\hline \multirow{2}{*}{$\begin{array}{l}\text { Dorsoventral Outlet } \\
\text { Diameter } \\
\text { /S1Body Breadth }\end{array}$} & 66.4 & 68.5 & 0.254 & 97 \\
\hline & 3.91 & 3.82 & 0.314 & 102 \\
\hline
\end{tabular}

*Indicates males and females exhibit statistical differences at $\alpha=0.035$. 
Table 2.8: Student's t-test results for differences between males and females in residuals from Reduced Major Axis regressions of pelvic size variables with $\mathrm{S1}$ body breadth

\begin{tabular}{|c|c|c|c|}
\hline \multirow[b]{2}{*}{ Large Cephalopelvic Proportions } & \multicolumn{3}{|c|}{ Birth Canal Centroid Size } \\
\hline & Mean Female Residual & Mean Male Residual & $\begin{array}{c}p \text {-value } \\
\text { (larger sex) }\end{array}$ \\
\hline Homo sapiens & -0.15 & -0.26 & $<0.001 *(\mathrm{f})$ \\
\hline Hylobates lar & 2.15 & -0.03 & $<0.001 *(\mathrm{f})$ \\
\hline Macaca mulatta & 0.16 & 0.12 & $<0.001 *(\mathrm{f})$ \\
\hline Nasalis larvatus & 0.17 & -7.34 & $<0.001 *(\mathrm{f})$ \\
\hline Papio anubis & 0.25 & 0.08 & $<0.001 *(\mathrm{f})$ \\
\hline Saimiri sciureus & 0.09 & -0.01 & $<0.001 *(\mathrm{f})$ \\
\hline \multicolumn{4}{|l|}{ Small Cephalopelvic Proportions } \\
\hline Gorilla gorilla & 0.15 & 0.14 & 0.703 \\
\hline Pan troglodytes & 0.17 & 0.11 & $<0.001 *(\mathrm{f})$ \\
\hline Alouatta seniculus & -0.35 & -0.35 & 0.756 \\
\hline & \multicolumn{3}{|c|}{ Inlet Circumference } \\
\hline Large Cephalopelvic Proportions & Mean Female Residual & Mean Male Residual & $\begin{array}{c}p \text {-value } \\
\text { (larger sex) }\end{array}$ \\
\hline Homo sapiens & -0.03 & -0.14 & $<0.001 *(\mathrm{f})$ \\
\hline Hylobates lar & 0.09 & 0.02 & $<0.001 *(\mathrm{f})$ \\
\hline Macaca mulatta & 0.19 & 0.09 & $<0.001 *(\mathrm{f})$ \\
\hline Nasalis larvatus & 0.12 & -0.07 & $<0.001 *(\mathrm{f})$ \\
\hline Papio anubis & 0.18 & 0.08 & $<0.001 *(\mathrm{f})$ \\
\hline Saimiri sciureus & 0.15 & -0.02 & $<0.001 *(\mathrm{f})$ \\
\hline \multicolumn{4}{|l|}{ Small Cephalopelvic Proportions } \\
\hline Gorilla gorilla & 0.21 & 0.17 & $0.009 *(\mathrm{f})$ \\
\hline Pan troglodytes & 0.19 & 0.12 & $<0.001 *(\mathrm{f})$ \\
\hline \multirow{2}{*}{ Alouatta seniculus } & -0.31 & -0.31 & 0.911 \\
\hline & \multicolumn{3}{|c|}{ Midplane Circumference } \\
\hline Large Cephalopelvic Proportions & Mean Female Residual & Mean Male Residual & $\begin{array}{c}p \text {-value } \\
\text { (larger sex) }\end{array}$ \\
\hline Homo sapiens & -0.1 & $\begin{array}{r}0.24 \\
\end{array}$ & $<0.001 *(\mathrm{f})$ \\
\hline Hylobates lar & 0.07 & 0.02 & $<0.001 *(\mathrm{f})$ \\
\hline Macaca mulatta & 0.22 & 0.16 & $<0.001 *(\mathrm{f})$ \\
\hline Nasalis larvatus & -0.05 & -0.17 & $0.001 *(\mathrm{f})$ \\
\hline Papio anubis & 0.2 & 0.08 & $<0.001 *(\mathrm{f})$ \\
\hline Saimiri sciureus & 0.13 & -0.01 & $<0.001 *(\mathrm{f})$ \\
\hline \multicolumn{4}{|l|}{ Small Cephalopelvic Proportions } \\
\hline Gorilla gorilla & 0.22 & 0.19 & $0.015^{*}(\mathrm{f})$ \\
\hline Pan troglodytes & 0.25 & 0.19 & $<0.001 *(\mathrm{f})$ \\
\hline \multirow[t]{2}{*}{ Alouatta seniculus } & -0.46 & -0.48 & 0.261 \\
\hline & \multicolumn{3}{|c|}{ Outlet Circumference } \\
\hline Large Cephalopelvic Proportions & Mean Female Residual & Mean Male Residual & $\begin{array}{c}p \text {-value } \\
\text { (larger sex) }\end{array}$ \\
\hline Homo sapiens & -0.06 & -0.22 & $<0.001 *(\mathrm{f})$ \\
\hline Hylobates lar & 0.09 & 0.04 & $<0.001 *(\mathrm{f})$ \\
\hline Macaca mulatta & 0.25 & 0.12 & $<0.001 *(\mathrm{f})$ \\
\hline Nasalis larvatus & 0.05 & -0.04 & $0.003 *(f)$ \\
\hline Papio anubis & 0.24 & 0.11 & $<0.001 *(\mathrm{f})$ \\
\hline Saimiri sciureus & 0.09 & -0.01 & $<0.001 *(\mathrm{f})$ \\
\hline \multicolumn{4}{|l|}{ Small Cephalopelvic Proportions } \\
\hline Gorilla gorilla & 0.23 & 0.21 & 0.261 \\
\hline Pan troglodytes & 0.28 & 0.22 & $<0.001 *(\mathrm{f})$ \\
\hline Alouatta seniculus & -0.33 & -0.34 & 0.969 \\
\hline
\end{tabular}


Table 2.8 (cot'd): Student's t-test results for differences between males and females in residuals from

Reduced Major Axis regressions of pelvic size variables with S1 body breadth

Transverse Inlet Diameter

\begin{tabular}{|c|c|c|c|}
\hline \multirow[b]{2}{*}{ Large Cephalopelvic Proportions } & \\
\hline & Mean Female Residual & Mean Male Residual & $\begin{array}{c}p \text {-value } \\
\text { (larger sex) }\end{array}$ \\
\hline Homo sapiens & 0.03 & -0.08 & $<0.001 *(\mathrm{f})$ \\
\hline Hylobates lar & 0.07 & -0.01 & $<0.001 *(\mathrm{f})$ \\
\hline Macaca mulatta & 0.16 & 0.08 & $<0.001 *(\mathrm{f})$ \\
\hline Nasalis larvatus & 0.17 & 0.05 & $<0.001 *(\mathrm{f})$ \\
\hline Papio anubis & 0.28 & 0.22 & $0.031 *(f)$ \\
\hline Saimiri sciureus & 0.16 & 0.02 & $<0.001 *(\mathrm{f})$ \\
\hline \multicolumn{4}{|l|}{ Small Cephalopelvic Proportions } \\
\hline Gorilla gorilla & 0.11 & 0.07 & $0.007 *(\mathrm{f})$ \\
\hline Pan troglodytes & 0.13 & 0.01 & $<0.001 *(\mathrm{f})$ \\
\hline \multirow[t]{2}{*}{ Alouatta seniculus } & -0.33 & -0.34 & 0.758 \\
\hline & \multicolumn{3}{|c|}{ Transverse Midplane Diameter } \\
\hline Large Cephalopelvic Proportions & Mean Female Residual & Mean Male Residual & $\begin{array}{c}p \text {-value } \\
\text { (larger sex) }\end{array}$ \\
\hline Homo sapiens & 0.12 & -0.08 & $<0.001 *(\mathrm{f})$ \\
\hline Hylobates lar & 0.11 & 0.05 & $0.015 *(\mathrm{f})$ \\
\hline Macaca mulatta & 0.21 & 0.17 & $0.026 *(f)$ \\
\hline Nasalis larvatus & 0.14 & -0.21 & $<0.001 *(\mathrm{f})$ \\
\hline Papio anubis & 0.15 & -0.05 & $0.009 *(\mathrm{f})$ \\
\hline Saimiri sciureus & 0.14 & 0.02 & $<0.001 *(\mathrm{f})$ \\
\hline \multicolumn{4}{|l|}{ Small Cephalopelvic Proportions } \\
\hline Gorilla gorilla & 0.12 & 0.19 & $0.026^{*}(\mathrm{~m})$ \\
\hline Pan troglodytes & 0.26 & 0.14 & 0.039 \\
\hline \multirow[t]{2}{*}{ Alouatta seniculus } & -0.33 & -0.38 & 0.078 \\
\hline & \multicolumn{3}{|c|}{ Transverse Outlet Diameter } \\
\hline Large Cephalopelvic Proportions & Mean Female Residual & Mean Male Residual & $\begin{array}{c}p \text {-value } \\
\text { (larger sex) }\end{array}$ \\
\hline Homo sapiens & 0.05 & -0.18 & $<0.001 *(\mathrm{f})$ \\
\hline Hylobates lar & 0.13 & 0.02 & $<0.001 *(\mathrm{f})$ \\
\hline Macaca mulatta & 0.18 & 0.07 & $<0.001 *(\mathrm{f})$ \\
\hline Nasalis larvatus & -0.09 & -0.42 & $<0.001 *(\mathrm{f})$ \\
\hline Papio anubis & 0.38 & 0.19 & $0.025^{*}(\mathrm{f})$ \\
\hline Saimiri sciureus & 0.1 & 0.03 & $<0.001 *(\mathrm{f})$ \\
\hline \multicolumn{4}{|l|}{ Small Cephalopelvic Proportions } \\
\hline Gorilla gorilla & 0.21 & 0.18 & 0.193 \\
\hline Pan troglodytes & 0.35 & 0.22 & $<0.001 *(\mathrm{f})$ \\
\hline \multirow[t]{2}{*}{ Alouatta seniculus } & -0.18 & -0.16 & 0.564 \\
\hline & \multicolumn{3}{|c|}{ Dorsoventral Inlet Diameter } \\
\hline Large Cephalopelvic Proportions & Mean Female Residual & Mean Male Residual & $\begin{array}{c}p \text {-value } \\
\text { (larger sex) }\end{array}$ \\
\hline Homo sapiens & -0.2 & -0.34 & $<0.001 *(\mathrm{f})$ \\
\hline Hylobates lar & 0.16 & 0.1 & $<0.001 *(\mathrm{f})$ \\
\hline Macaca mulatta & 0.22 & 0.11 & $<0.001 *(\mathrm{f})$ \\
\hline Nasalis larvatus & 0.19 & -0.06 & $<0.001 *(\mathrm{f})$ \\
\hline Papio anubis & 0.19 & -0.01 & $<0.001 *(\mathrm{f})$ \\
\hline Saimiri sciureus & 0.17 & -0.02 & $<0.001 *(\mathrm{f})$ \\
\hline \multicolumn{4}{|l|}{ Small Cephalopelvic Proportions } \\
\hline Gorilla gorilla & 0.3 & 0.26 & 0.048 \\
\hline Pan troglodytes & 0.27 & 0.21 & $0.015^{*}(\mathrm{f})$ \\
\hline Alouatta seniculus & -0.25 & -0.26 & 0.687 \\
\hline
\end{tabular}


Table 2.8 (cot'd): Student's t-test results for differences between males and females in residuals from Reduced Major Axis regressions of pelvic size variables with S1 body breadth

Dorsoventral Midplane Diameter

\begin{tabular}{|c|c|c|c|}
\hline Large Cephalopelvic Proportions & Mean Female Residual & Mean Male Residual & $\begin{array}{c}p \text {-value } \\
\text { (larger sex) }\end{array}$ \\
\hline Homo sapiens & -0.19 & -0.28 & $<0.001 *(\mathrm{f})$ \\
\hline Hylobates lar & 0.10 & 0.06 & $0.002 *(f)$ \\
\hline Macaca mulatta & 0.20 & 0.17 & $0.008 *(f)$ \\
\hline Nasalis larvatus & 0.07 & -0.04 & $0.004 *(f)$ \\
\hline Papio anubis & 0.15 & 0.06 & $0.009 *(\mathrm{f})$ \\
\hline Saimiri sciureus & 0.15 & -0.01 & $<0.001 *(\mathrm{f})$ \\
\hline \multicolumn{4}{|l|}{ Small Cephalopelvic Proportions } \\
\hline Gorilla gorilla & 0.25 & 0.24 & 0.349 \\
\hline Pan troglodytes & 0.24 & 0.20 & $0.018 *(f)$ \\
\hline \multirow[t]{2}{*}{ Alouatta seniculus } & -0.45 & -0.48 & 0.392 \\
\hline & \multicolumn{3}{|c|}{ Dorsoventral Outlet Diameter } \\
\hline Large Cephalopelvic Proportions & Mean Female Residual & Mean Male Residual & $\begin{array}{c}p \text {-value } \\
\text { (larger sex) }\end{array}$ \\
\hline Homo sapiens & -0.05 & -0.20 & $<0.001 *(\mathrm{f})$ \\
\hline Hylobates lar & 0.09 & 0.04 & $<0.001 *(\mathrm{f})$ \\
\hline Macaca mulatta & 0.20 & 0.16 & $0.007 *(\mathrm{f})$ \\
\hline Nasalis larvatus & -0.03 & -0.02 & 0.875 \\
\hline Papio anubis & 0.08 & 0.06 & 0.645 \\
\hline Saimiri sciureus & 0.06 & 0.05 & 0.763 \\
\hline \multicolumn{4}{|l|}{ Small Cephalopelvic Proportions } \\
\hline Gorilla gorilla & 0.24 & 0.18 & $0.009 *(\mathrm{f})$ \\
\hline Pan troglodytes & 0.24 & 0.18 & $0.002 *(\mathrm{f})$ \\
\hline Alouatta seniculus & -0.48 & -0.52 & 0.089 \\
\hline
\end{tabular}




\begin{tabular}{|c|c|c|}
\hline \multicolumn{3}{|c|}{ Table 2.9: Dimorphism Index Ranks } \\
\hline Species & $\begin{array}{c}\text { Dimorphism Index for } \\
\text { relative birth canal } \\
\text { centroid size* }\end{array}$ & $\begin{array}{l}\text { Cephalopelvic } \\
\text { Proportions }\end{array}$ \\
\hline Nasalis larvatus & 130 & large \\
\hline Papio anubis & 121 & large \\
\hline Homo sapiens & 117 & large \\
\hline Saimiri sciureus & 110 & large \\
\hline Pan troglodytes & 106 & small \\
\hline Hylobates lar & 105 & large \\
\hline Macaca mulatta & 105 & large \\
\hline Gorilla gorilla & 103 & small \\
\hline Alouatta seniculus & 100 & small \\
\hline Species & $\begin{array}{c}\text { dimorphism index for } \\
\text { relative inlet } \\
\text { circumference* }\end{array}$ & $\begin{array}{l}\text { Cephalopelvic } \\
\text { Proportions }\end{array}$ \\
\hline Nasalis larvatus & 122 & large \\
\hline Saimiri sciureus & 120 & large \\
\hline Homo sapiens & 114 & large \\
\hline Papio anubis & 112 & large \\
\hline Macaca mulatta & 111 & large \\
\hline Pan troglodytes & 109 & small \\
\hline Hylobates lar & 108 & large \\
\hline Gorilla gorilla & 107 & small \\
\hline Alouatta seniculus & 100 & small \\
\hline Species & $\begin{array}{c}\text { dimorphism index for } \\
\text { relative midplane } \\
\text { circumference* }\end{array}$ & $\begin{array}{l}\text { Cephalopelvic } \\
\text { Proportions }\end{array}$ \\
\hline Homo sapiens & 117 & large \\
\hline Saimiri sciureus & 115 & large \\
\hline Nasalis larvatus & 114 & large \\
\hline Papio anubis & 112 & large \\
\hline Macaca mulatta & 107 & large \\
\hline Pan troglodytes & 107 & small \\
\hline Gorilla gorilla & 106 & small \\
\hline Hylobates lar & 105 & large \\
\hline Alouatta seniculus & 103 & small \\
\hline Species & $\begin{array}{c}\text { dimorphism index for } \\
\text { relative outlet } \\
\text { circumference* }\end{array}$ & $\begin{array}{l}\text { Cephalopelvic } \\
\text { Proportions }\end{array}$ \\
\hline Homo sapiens & 119 & large \\
\hline Papio anubis & 115 & large \\
\hline Macaca mulatta & 114 & large \\
\hline Nasalis larvatus & 111 & large \\
\hline Saimiri sciureus & 111 & large \\
\hline Pan troglodytes & 107 & small \\
\hline Hylobates lar & 106 & large \\
\hline Gorilla gorilla & 104 & small \\
\hline Alouatta seniculus & 100 & small \\
\hline
\end{tabular}

${ }^{*}$ Calculated as $\{[($ female mean $) /($ male mean $)] * 100\}$. 


\begin{tabular}{|c|c|c|}
\hline \multicolumn{3}{|c|}{ Table 2.9 (cot'd): Dimorphism Index Ranks } \\
\hline Species & $\begin{array}{c}\text { dimorphism index for relative } \\
\text { mediolateral diameter of the } \\
\text { inlet* }\end{array}$ & $\begin{array}{l}\text { Cephalopelvic } \\
\text { Proportions }\end{array}$ \\
\hline Saimiri sciureus & 115 & large \\
\hline Homo sapiens & 114 & large \\
\hline Nasalis larvatus & 114 & large \\
\hline Pan troglodytes & 114 & small \\
\hline Macaca mulatta & 110 & large \\
\hline Hylobates lar & 109 & large \\
\hline Papio anubis & 109 & large \\
\hline Gorilla gorilla & 106 & small \\
\hline Alouatta seniculus & 101 & small \\
\hline Species & $\begin{array}{c}\text { dimorphism index for relative } \\
\text { mediolateral diameter of the } \\
\text { midplane* }\end{array}$ & $\begin{array}{l}\text { Cephalopelvic } \\
\text { Proportions }\end{array}$ \\
\hline Nasalis larvatus & 138 & large \\
\hline Homo sapiens & 134 & large \\
\hline Papio anubis & 121 & large \\
\hline Pan troglodytes & 116 & small \\
\hline Saimiri sciureus & 113 & large \\
\hline Gorilla gorilla & 112 & small \\
\hline Hylobates lar & 111 & large \\
\hline Alouatta seniculus & 105 & small \\
\hline Macaca mulatta & 104 & large \\
\hline Species & $\begin{array}{c}\text { dimorphism index for relative } \\
\text { mediolateral diameter of the } \\
\text { outlet* }\end{array}$ & $\begin{array}{l}\text { Cephalopelvic } \\
\text { Proportions }\end{array}$ \\
\hline Nasalis larvatus & 143 & large \\
\hline Homo sapiens & 124 & large \\
\hline Papio anubis & 124 & large \\
\hline Macaca mulatta & 113 & large \\
\hline Hylobates lar & 111 & large \\
\hline Pan troglodytes & 110 & small \\
\hline Saimiri sciureus & 108 & large \\
\hline Gorilla gorilla & 101 & small \\
\hline Alouatta seniculus & 99 & small \\
\hline Species & $\begin{array}{c}\text { dimorphism index for relative } \\
\text { dorsoventral diameter of the } \\
\text { inlet* }\end{array}$ & $\begin{array}{l}\text { Cephalopelvic } \\
\text { Proportions }\end{array}$ \\
\hline Nasalis larvatus & 126 & large \\
\hline Papio anubis & 121 & large \\
\hline Saimiri sciureus & 120 & large \\
\hline Homo sapiens & 117 & large \\
\hline Macaca mulatta & 111 & large \\
\hline Gorilla gorilla & 107 & small \\
\hline Hylobates lar & 106 & large \\
\hline Pan troglodytes & 106 & small \\
\hline Alouatta seniculus & 101 & small \\
\hline
\end{tabular}

*Calculated as $\left\{\left[(\right.\right.$ female mean $) /($ male mean $\left.){ }^{*} 100\right\}$. 


\begin{tabular}{lcl}
\hline Table 2.9 (cot'd): Dimorphism Index Ranks & $\begin{array}{c}\text { dimorphism index for relative } \\
\text { dorsoventral diameter of the } \\
\text { midplane* }\end{array}$ & $\begin{array}{l}\text { Cephalopelvic } \\
\text { Proportions }\end{array}$ \\
\hline Saimiri sciureus & 116 & large \\
Homo sapiens & 112 & large \\
Nasalis larvatus & 107 & large \\
Papio anubis & 106 & large \\
Pan troglodytes & 105 & small \\
Gorilla gorilla & 104 & small \\
Hylobates lar & 104 & large \\
Alouatta seniculus & 101 & small \\
Macaca mulatta & 101 & large \\
\hline & dimorphism index for relative & Cephalopelvic \\
Species & dorsoventral diameter of the & Proportions \\
& outlet* & large \\
\hline Saimiri sciureus & 119 & large \\
Homo sapiens & 119 & small \\
Gorilla gorilla & 110 & small \\
Pan troglodytes & 108 & large \\
Nasalis larvatus & 107 & large \\
Papio anubis & 106 & large \\
Hylobates lar & 105 & large \\
Macaca mulatta & 105 & small \\
Alouatta seniculus & 102 & \\
\hline
\end{tabular}

*Calculated as $\left\{[(\text { female mean }) /(\text { male mean })]^{*} 100\right\}$.

\begin{tabular}{lccc}
\hline \multicolumn{4}{l}{ Table 2.10: Results for Discriminant } \\
intranction analyses of the influence of sex on \\
\hline Large Cephalopelvic Proportions & $\begin{array}{c}\text { Procrustes } \\
\text { Distance }\end{array}$ & $\begin{array}{c}\text { Mahalanobis } \\
\text { Distance }\end{array}$ & p-value \\
\hline Homo sapiens & 0.0919 & 4.896 & $<0.0001^{*}$ \\
Hylobates lar & 0.0402 & 3.156 & $<0.0001^{*}$ \\
Macaca mulatta & 0.1086 & 5.378 & $<0.0001^{*}$ \\
Nasalis larvatus & 0.1057 & 19.592 & 0.4145 \\
Papio anubis & 0.0847 & 5.588 & 0.9023 \\
Saimiri sciureus & 0.0842 & 5.561 & $<0.0001^{*}$ \\
\hline Small Cephalopelvic Proportions & Procrustes & Mahalanobis & \multirow{2}{*}{ p-value } \\
\hline Alouatta seniculus & Distance & Distance & \\
Gorilla gorilla & 0.0316 & 4.470 & 0.9871 \\
Pan troglodytes & 0.0641 & 4.923 & $<0.0001^{*}$ \\
\hline
\end{tabular}

*Indicates males and females exhibit statistical differences in overall birth canal form at $\alpha=0.035$. 


\begin{tabular}{lcccccc}
\hline \multicolumn{6}{l}{ Table 2.11: Student's tests for dimorphism in birth canal shape along each Principal Component; } \\
\hline \multirow{2}{*}{ Large Proportions } & $\begin{array}{c}\text { p-value for } \\
\text { PC1 }\end{array}$ & $\begin{array}{c}\text { Variation } \\
\text { explained }\end{array}$ & $\begin{array}{c}\text { p-value for } \\
\text { PC2 }\end{array}$ & $\begin{array}{c}\text { Variation } \\
\text { explained }\end{array}$ & $\begin{array}{c}\text { p-value for } \\
\text { PC3 }\end{array}$ & $\begin{array}{c}\text { Variation } \\
\text { explained }\end{array}$ \\
\hline Homo sapiens & $<0.001^{*}$ & $30 \%$ & $0.029^{*}$ & $14 \%$ & 0.227 & $11 \%$ \\
Hylobates lar & $<0.001^{*}$ & $25 \%$ & $<0.001^{*}$ & $12 \%$ & 0.049 & $10 \%$ \\
Macaca mulatta & $<0.001^{*}$ & $38 \%$ & 0.255 & $12 \%$ & 0.074 & $11 \%$ \\
Nasalis larvatus & $<0.001^{*}$ & $36 \%$ & $0.005^{*}$ & $24 \%$ & 0.328 & $11 \%$ \\
Papio anubis & $0.002^{*}$ & $32 \%$ & 0.191 & $19 \%$ & 0.097 & $12 \%$ \\
Saimiri sciureus & $0.001^{*}$ & $28 \%$ & $<0.001^{*}$ & $19 \%$ & $<0.001^{*}$ & $13 \%$ \\
\hline \multirow{2}{*}{ Small Proportions } & p-value for & Variation & p-value for & Variation & p-value for & Variation \\
& PC1 & explained & PC2 & explained & PC3 & explained \\
\hline Alouatta seniculus & 0.818 & $34 \%$ & $0.026^{*}$ & $11 \%$ & 0.535 & $10 \%$ \\
Gorilla gorilla & $<0.001^{*}$ & $23 \%$ & $<0.001^{*}$ & $18 \%$ & $<0.001 *$ & $11 \%$ \\
Pan troglodytes & $<0.001^{*}$ & $22 \%$ & 0.036 & $14 \%$ & 0.136 & $11 \%$ \\
\hline
\end{tabular}

Firth canal shape defined as individuals' Principal Components scores of PCAs of intraspecies birth canal landmark data.

*Indicates males and females exhibit statistical differences in principal components scores (significant differences quantified using Student's t-tests) at $\alpha=0.035$.

\begin{tabular}{lcc}
\hline \multirow{2}{*}{$\begin{array}{l}\text { Table 2.12: Euclidean Distance Matrix Analyses FORM test of male and } \\
\text { female differences in overall birth canal shape within }\end{array}$} & $\begin{array}{c}\text { becies } \\
\text { birth canal } \\
\text { form } \\
\text { (unscaled) }\end{array}$ & $\begin{array}{c}\text { birth canal form } \\
\text { (scaled to geometric } \\
\text { mean) }\end{array}$ \\
\cline { 2 - 3 } Large Cephalopelvic Proportions & p-value & p-value \\
\hline Homo sapiens & $<0.001^{*}$ & $<0.001^{*}$ \\
Hylobates lar & $0.007^{*}$ & $0.003^{*}$ \\
Macaca mulatta & $<0.001^{*}$ & $<0.001^{*}$ \\
Nasalis larvatus & $<0.001^{*}$ & $<0.001^{*}$ \\
Papio anubis & $0.009^{*}$ & $0.014^{*}$ \\
Saimiri sciureus & $<0.001^{*}$ & $<0.001^{*}$ \\
\hline Small Cephalopelvic Proportions & p-value & p-value \\
\hline Alouatta seniculus & 0.337 & 0.332 \\
Gorilla gorilla & $<0.001^{*}$ & $<0.001^{*}$ \\
Pan troglodytes & $0.006^{*}$ & $0.007^{*}$ \\
\hline
\end{tabular}

*Indicates males and females exhibit statistical differences in overall birth canal form at $\alpha=0.035$. 


\begin{tabular}{|c|c|c|c|c|c|c|}
\hline \multirow[b]{2}{*}{ Large Proportions } & \multicolumn{2}{|c|}{ Inlet shape } & \multicolumn{2}{|c|}{ Midplane Shape } & \multicolumn{2}{|c|}{ Outlet Shape } \\
\hline & $\mathbf{F}$ & $\mathbf{M}$ & $\mathbf{F}$ & M & $\mathbf{F}$ & M \\
\hline Homo sapiens & 1.10 & 1.14 & 0.82 & 0.69 & 1.11 & 1.07 \\
\hline Hylobates lar & 0.73 & 0.71 & 0.59 & 0.56 & 0.79 & 0.75 \\
\hline Macaca mulatta & 0.75 & 0.76 & 0.56 & 0.55 & 0.70 & 0.65 \\
\hline Nasalis larvatus & 0.77 & 0.86 & 0.59 & 0.45 & 0.62 & 0.46 \\
\hline Papio anubis & 0.85 & 0.95 & 0.54 & 0.47 & 0.84 & 0.72 \\
\hline Saimiri sciureus & 0.85 & 0.88 & 0.60 & 0.62 & 0.92 & 1.02 \\
\hline Small Proportions & $\mathbf{F}$ & $\mathbf{M}$ & $\mathbf{F}$ & $\mathbf{M}$ & $\mathbf{F}$ & $\mathbf{M}$ \\
\hline Gorilla gorilla & 0.72 & 0.73 & 0.56 & 0.52 & 0.95 & 1.03 \\
\hline Pan troglodytes & 0.74 & 0.69 & 0.58 & 0.53 & 1.00 & 0.99 \\
\hline Alouatta seniculus & 0.70 & 0.70 & 0.59 & 0.57 & 0.82 & 0.85 \\
\hline
\end{tabular}

*Measured as $\{$ (mediolateral dimension of pelvic plane)/(anteroposterior dimension of pelvic plane)\}.

\begin{tabular}{lc}
\hline Table 2.14: Shape overlap between sexes* \\
\hline Large Proportions & Percent of Females in Male Shape Space \\
\hline Homo sapiens & $10 \%$ \\
Hylobates lar & $33 \%$ \\
Macaca mulatta & $10 \%$ \\
Nasalis larvatus & $0 \%$ \\
Papio anubis & $0 \%$ \\
Saimiri sciureus & $39 \%$ \\
\hline Small Proportions & Percent of Females in Male Shape Space \\
\hline Gorilla gorilla & $38 \%$ \\
Pan troglodytes & $79 \%$ \\
Alouatta seniculus & $67 \%$ \\
\hline
\end{tabular}

*Calculated as the percentage of females falling within the male distribution along PC1 and PC2 in an interspecies Principal Components Analysis. 


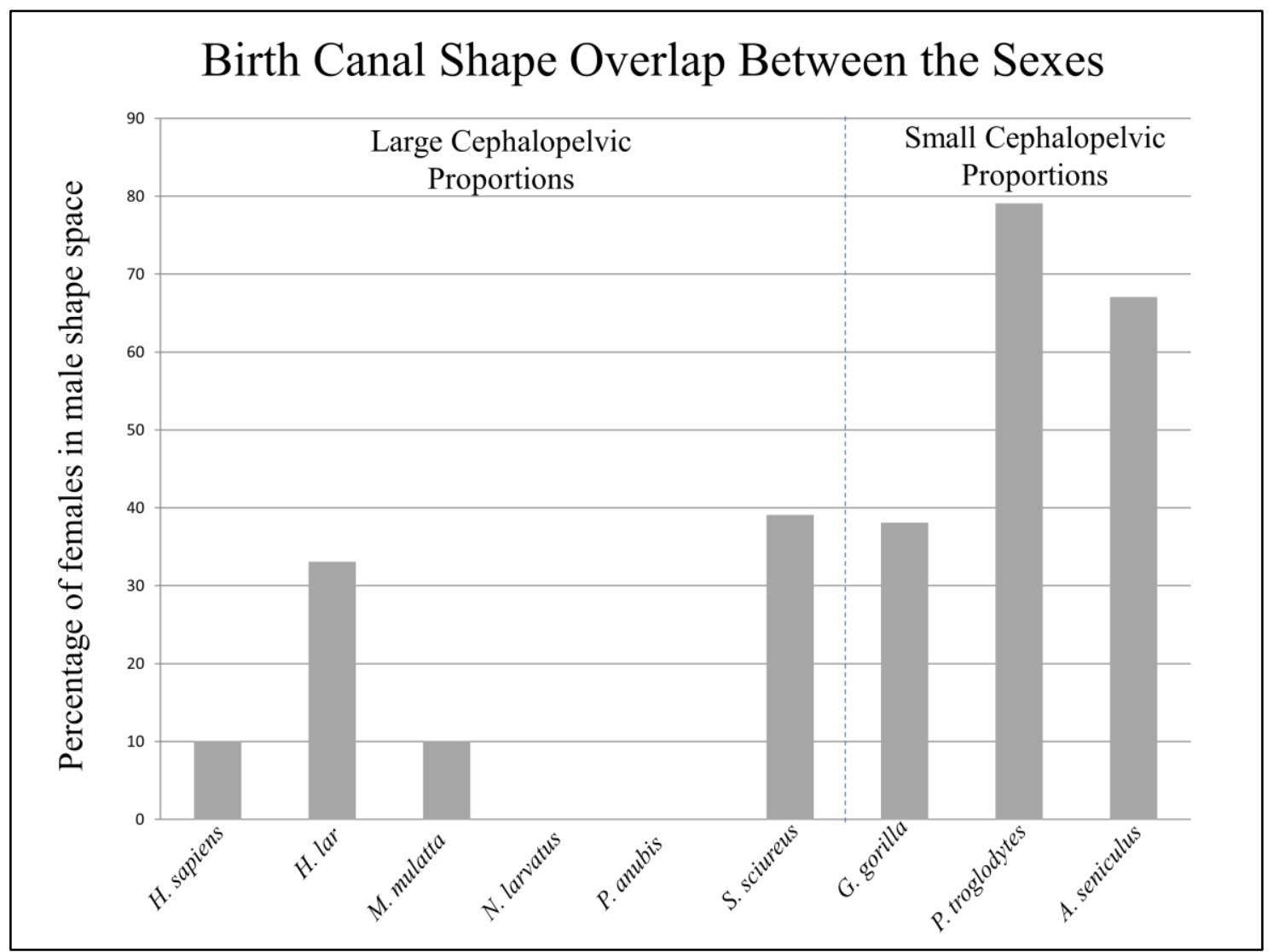

Figure 2.3: Birth canal shape overlap between the sexes, calculated as the percentage of females falling within the male distribution along PC1 and PC2 in an interspecies Principal Components Analysis. 


\section{Homo sapiens: Principal Components Analysis of Birth Canal Landmark Data}

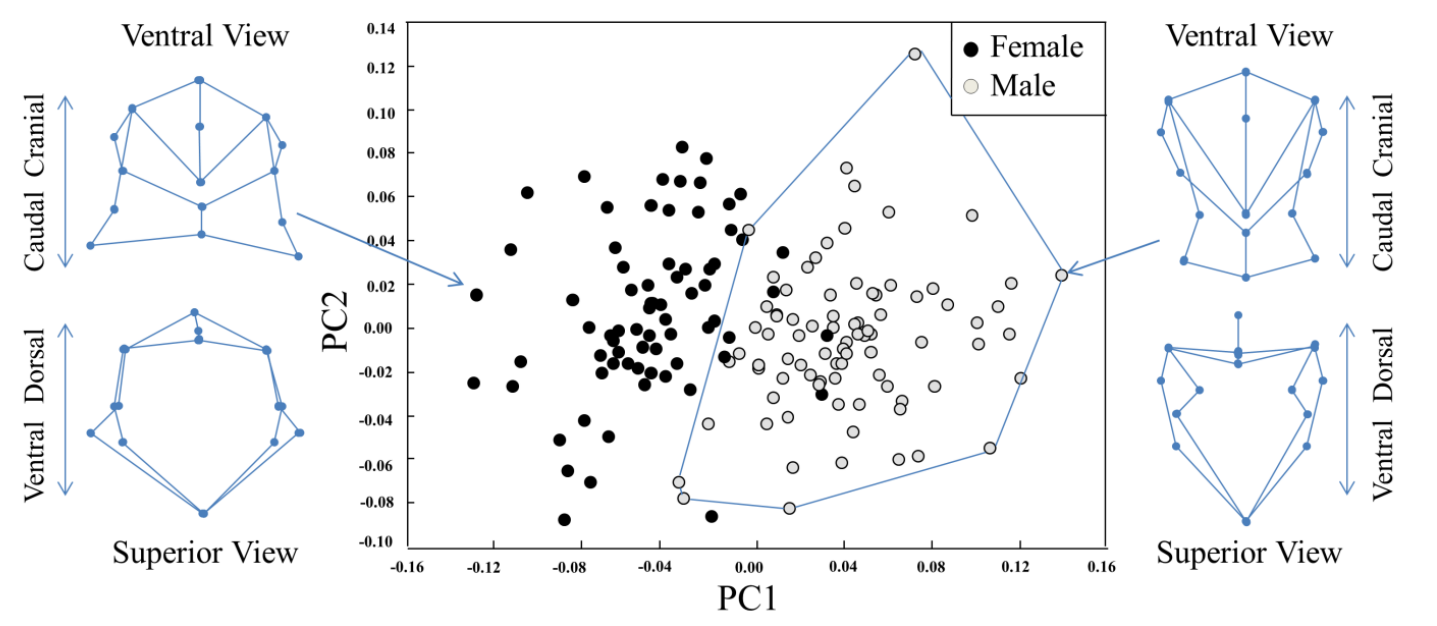

Figure 2.4a: Principal components analysis of birth canal shape in Homo sapiens. Note the diverging distributions of males and females along Axis 1.

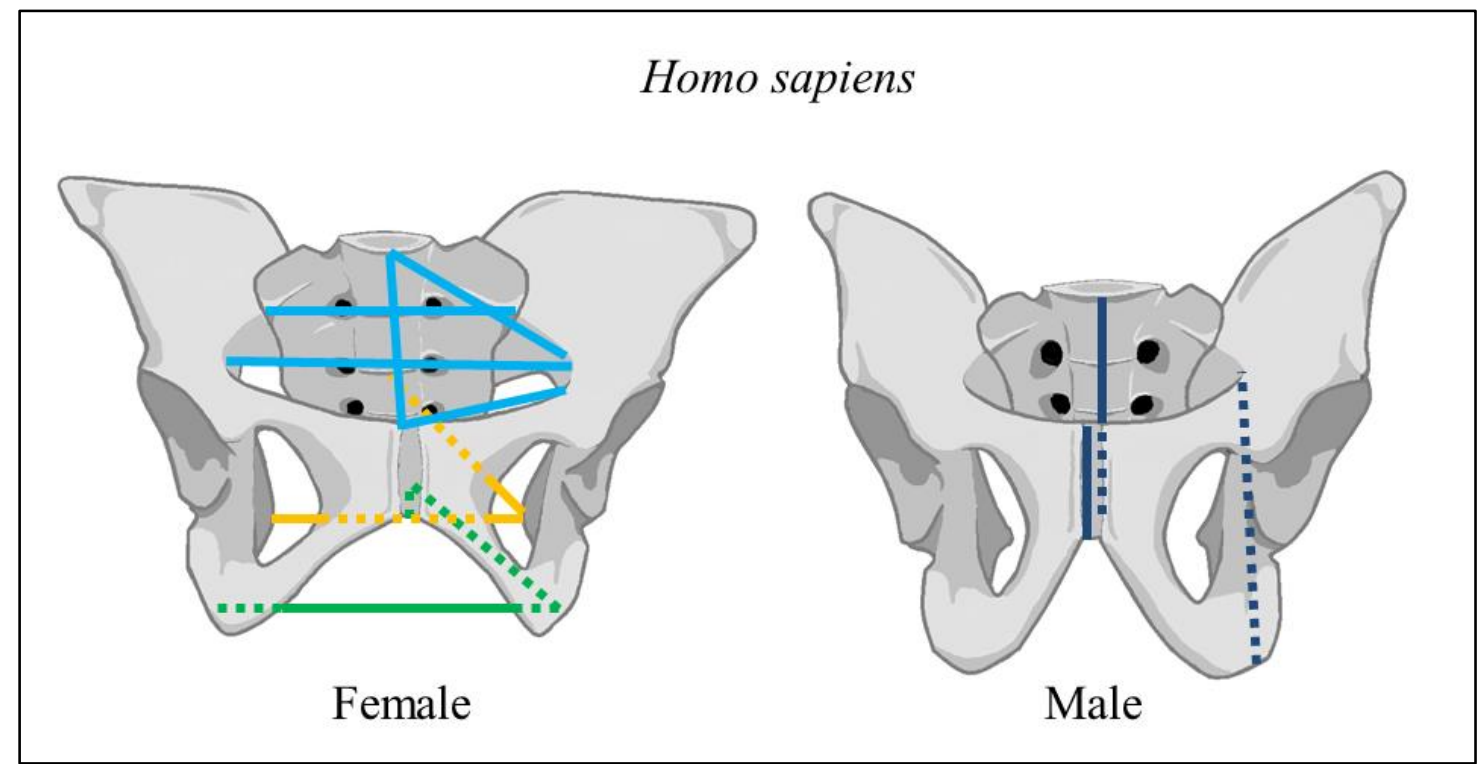

Figure 2.4b: Results of FORM procedure to obtain dimorphic linear distances in the pelvis of Homo sapiens when pelves are scaled to each individual's geometric mean (body size proxy). Light blue represents linear distances which are greater in females than males in the inlet, yellow represents distances which are greater in females than males in the midplane, and green represents linear distances which are greater in females than males in the outlet. Dark blue represents linear distances which are relatively greater in males compared to females. Only non-overlapping distances are included. 


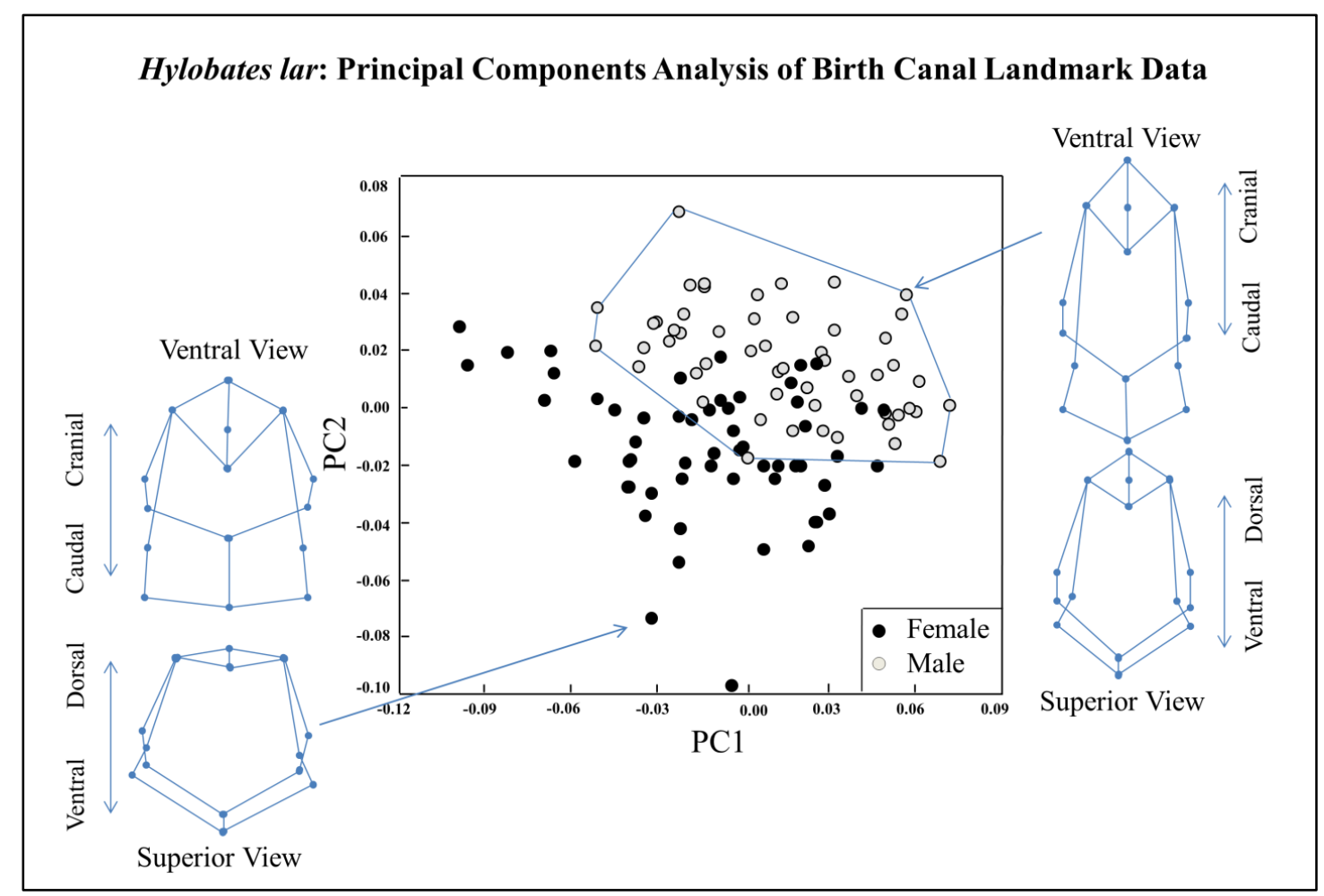

Figure 2.5a: Principal components analysis of birth canal shape in Hylobates lar. Note the diverging distributions of males and females along Axis 1 and Axis 2.

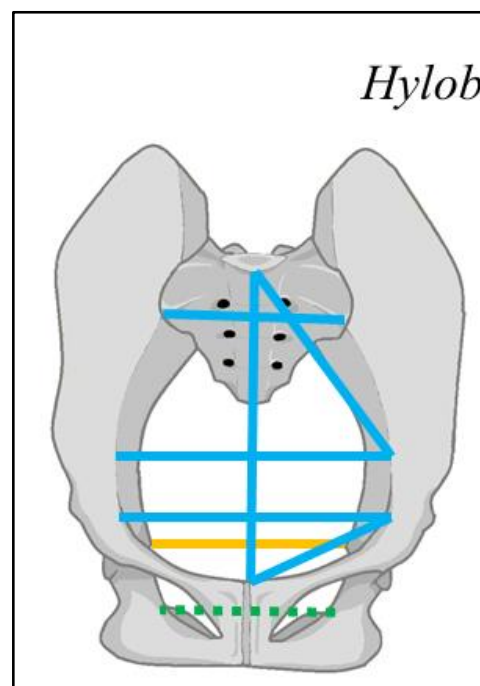

Female

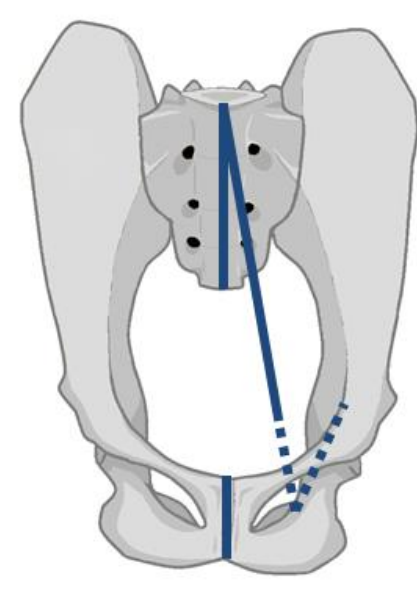

Male
Figure 2.5b: Results of FORM procedure to obtain dimorphic linear distances in the pelvis of Hylobates lar when pelves are scaled to each individual's geometric mean. Light blue represents distances which are greater in females than males in the inlet, yellow represents distances which are greater in females in the midplane, and green represents distances which are greater in females in the

outlet. Dark blue represents linear distances which are relatively greater in males. Only non-overlapping distances are included. 


\section{Macaca mulatta: Principal Components Analysis of Birth Canal Landmark Data}

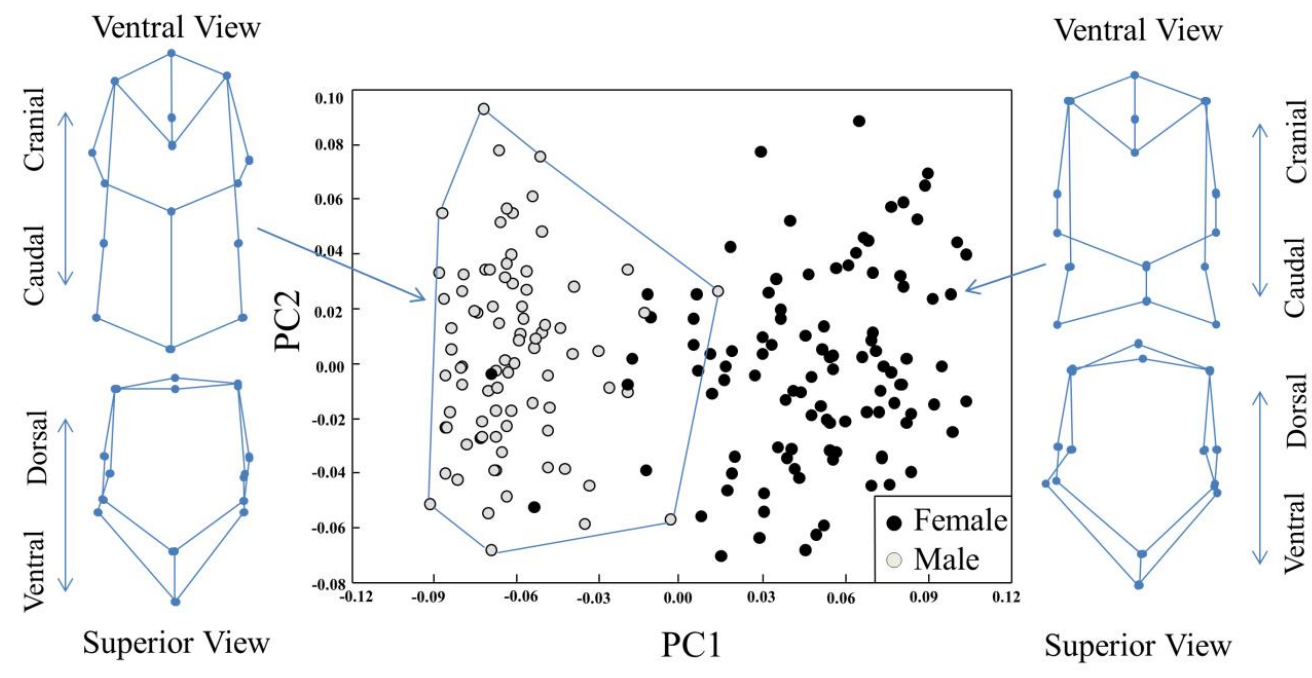

Figure 2.6a: Principal components analysis of birth canal shape in Macaca mulatta. Note the diverging distributions of males and females along Axis 1.

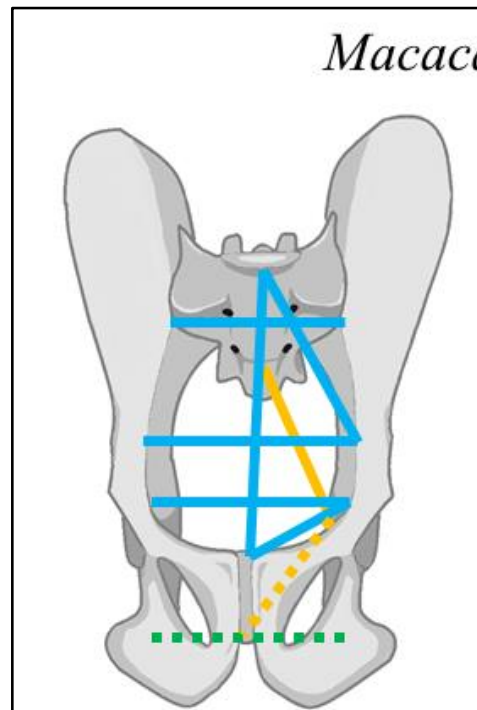

Female

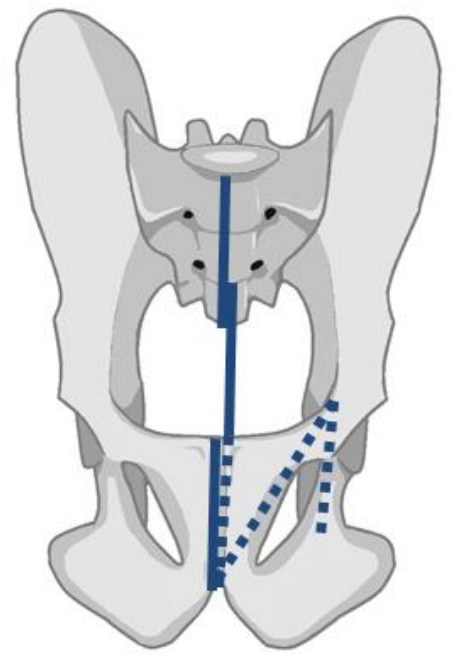

Male
Figure 2.6b: Graphical representation of results of Euclidean Distance Matrix Analysis's FORM procedure to obtain dimorphic linear distances in the pelvis of Macaca mulatta when pelves are scaled to each individual's geometric mean. Light blue represents distances which are greater in females than males in the inlet, yellow represents distances which are

greater in females in the midplane, and green represents distances which are greater in females in the outlet. Dark blue represents linear distances which are relatively greater in males. Only non-overlapping distances are included. 


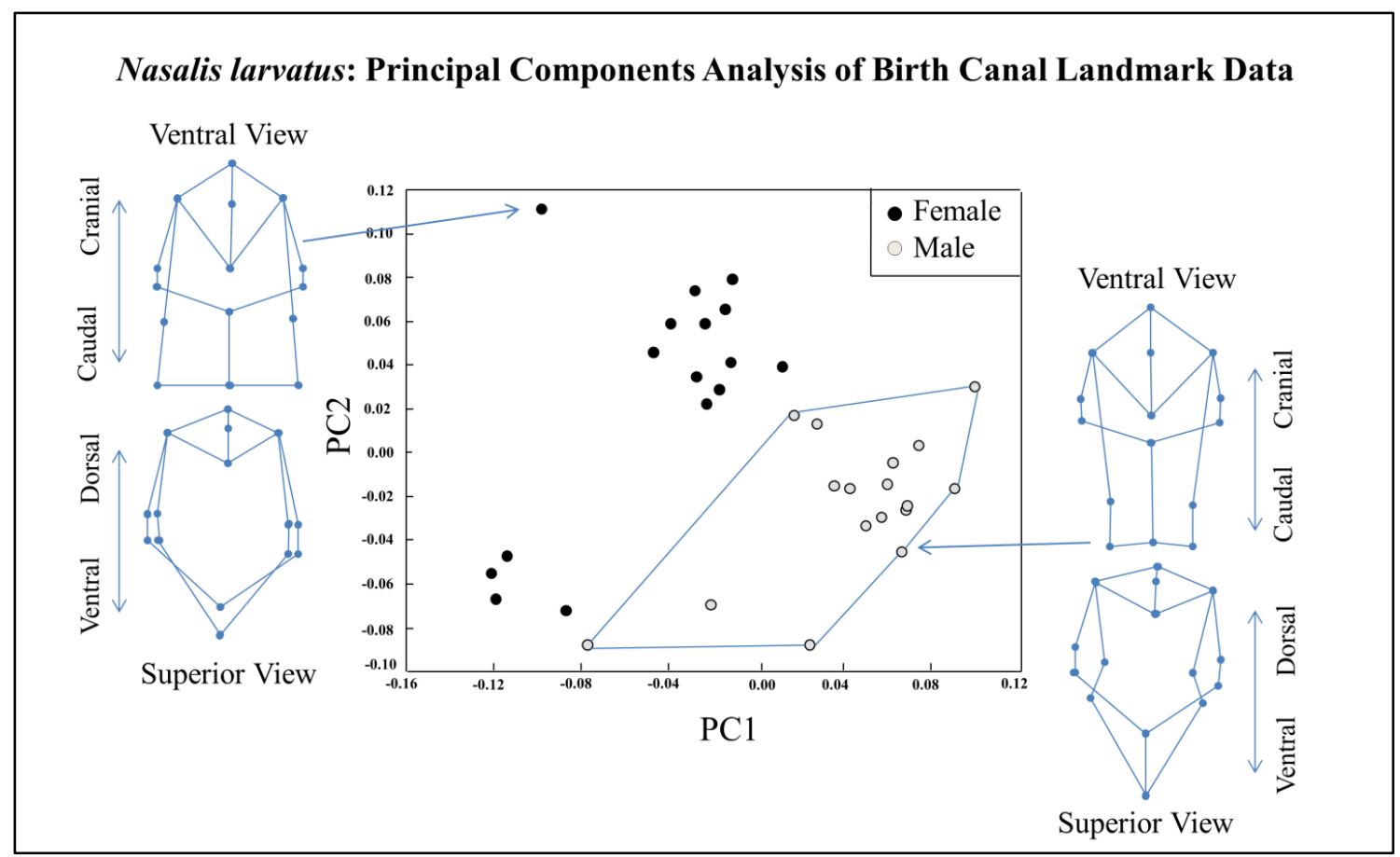

Figure 2.7a: Principal components analysis of birth canal shape in Nasalis larvatus. Note the diverging distributions of males and females along Axis 1 and Axis 2.

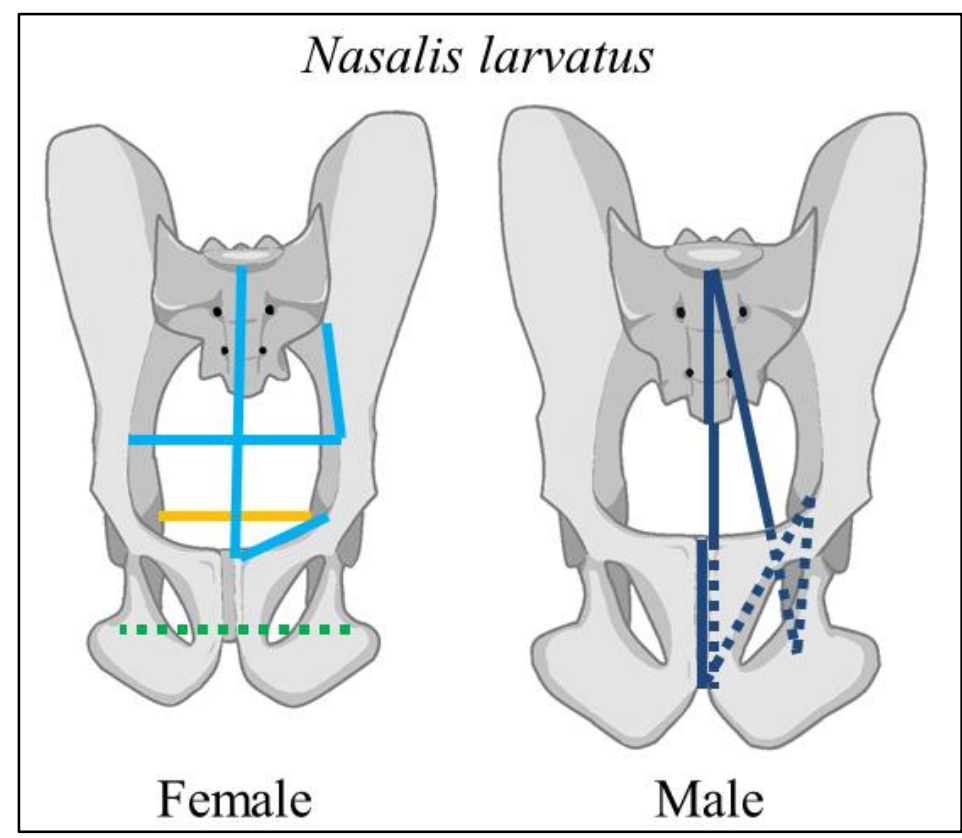

distances which are relatively greater in males. Only non-overlapping distances are included.
Figure 2.7b: Results of FORM procedure to obtain dimorphic linear distances in the pelvis of Nasalis larvatus when pelves are scaled to each individual's geometric mean. Light blue represents distances which are greater in females than males in the inlet, yellow represents distances which are greater in females in the midplane, and green represents distances which are greater in females in the outlet. Dark blue represents linear 
Papio anubis: Principal Components Analysis of Birth Canal Landmark Data

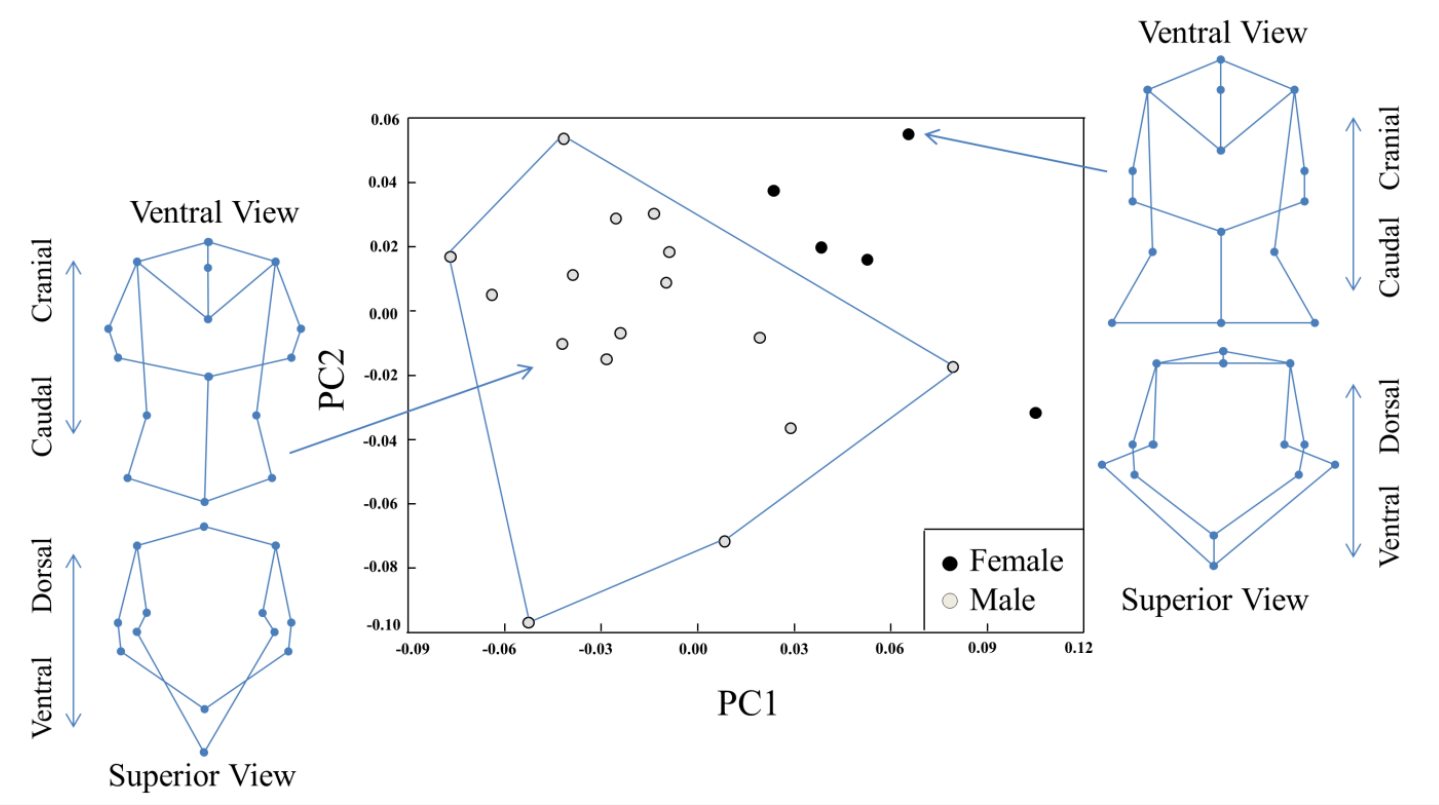

Figure 2.8a: Principal components analysis of birth canal shape in Papio anubis. Note the diverging distributions of males and females along Axis 1.

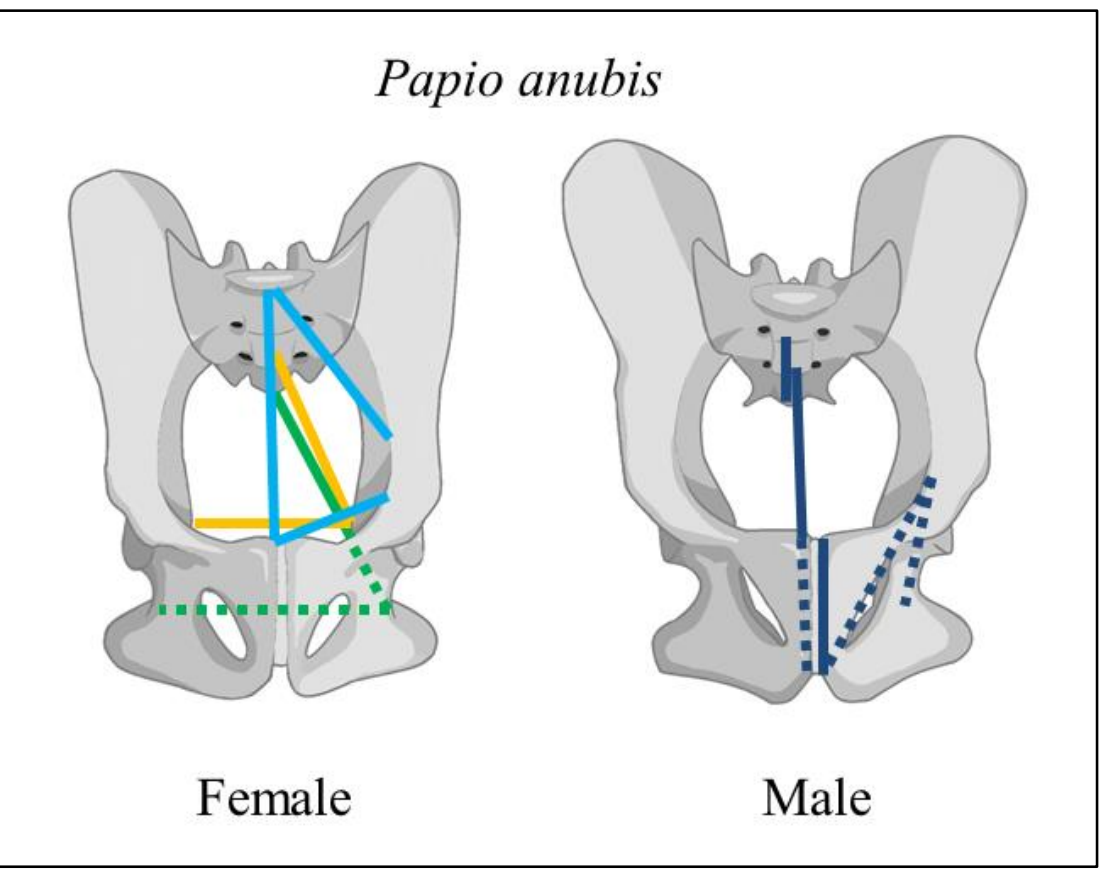

Figure 2.8b:

Results of FORM procedure to obtain dimorphic distances in the pelvis of Papio anubis when pelves are scaled to geometric mean. Light blue represents distances which are greater in females than males in the inlet, yellow represents distances which are greater in females in the midplane, and green represents distances greater in females in the outlet. Dark blue represents linear distances greater in males. Only non-overlapping distances are included. 


\section{Saimiri sciureus: Principal Components Analysis of Birth Canal Landmark Data}

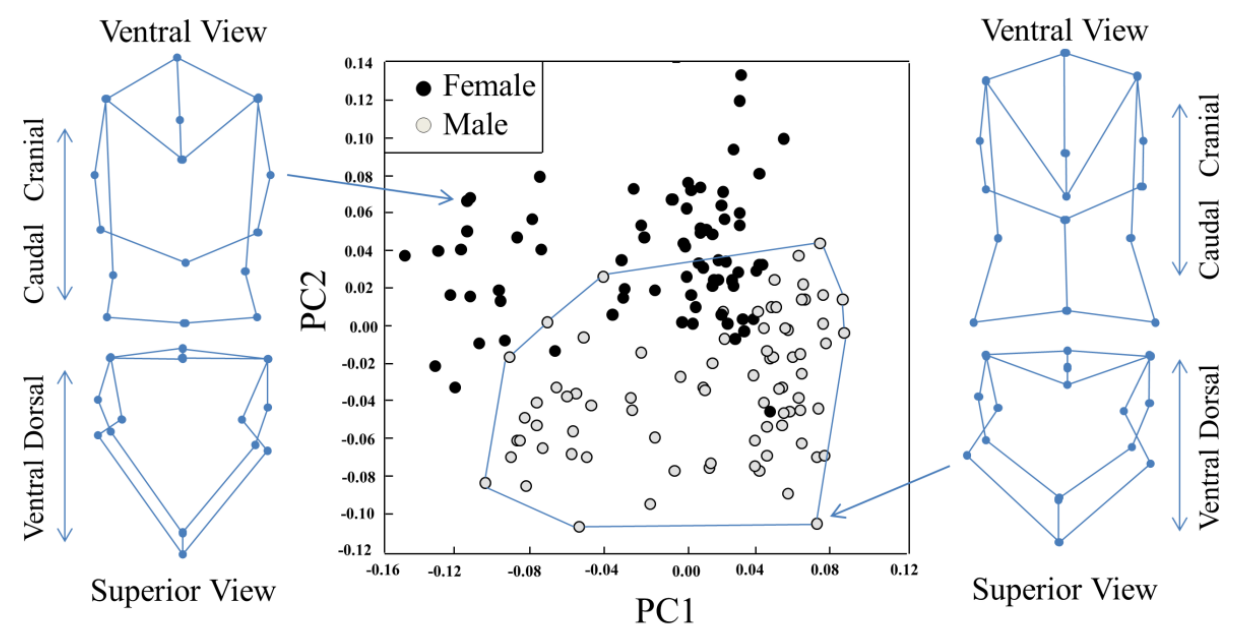

Figure 2.9a: Principal components analysis of birth canal shape in Saimiri sciureus. Note the diverging distributions of males and females along Axis 1 and Axis 2.

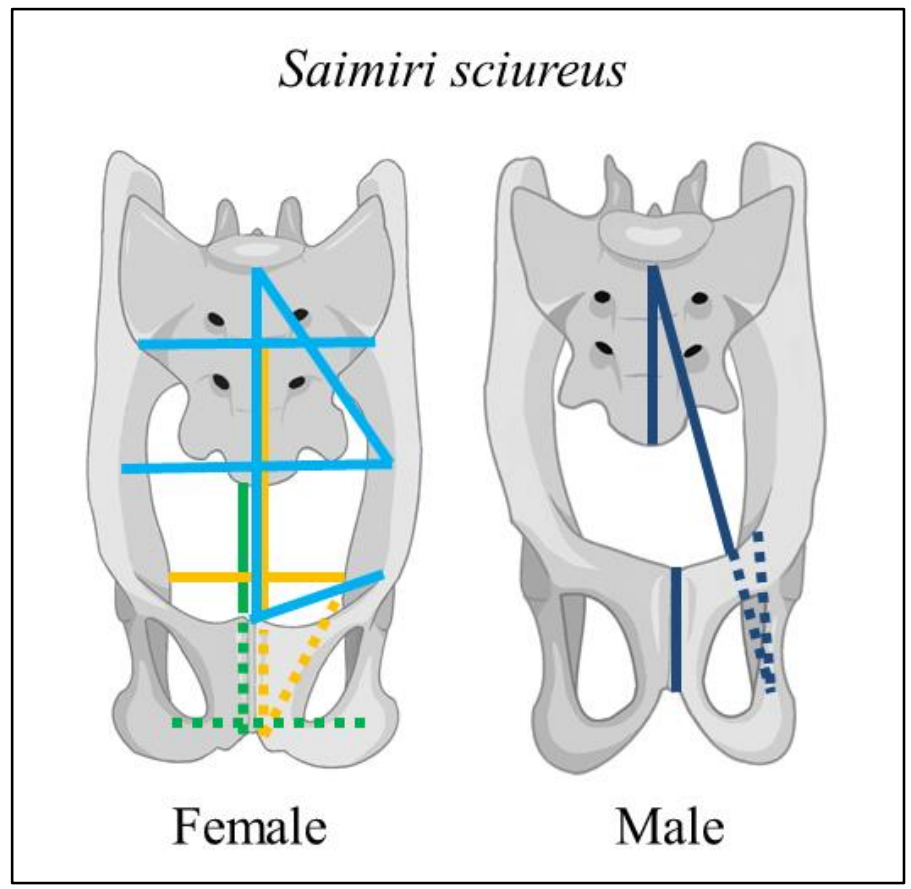

Figure 2.9b: Graphical representation of results of Euclidean Distance Matrix Analysis's FORM procedure to obtain dimorphic distances in the pelvis of Saimiri sciureus when pelves are scaled to geometric mean. Light blue represents distances which are greater in females than males in the inlet, yellow represents distances which are greater in females in the midplane, and green represents distances greater in females in the outlet. Dark blue represents linear distances relatively greater in males than females. Only non-overlapping distances are included. 


\section{Gorilla gorilla: Principal Components Analysis of Birth Canal Landmark Data}

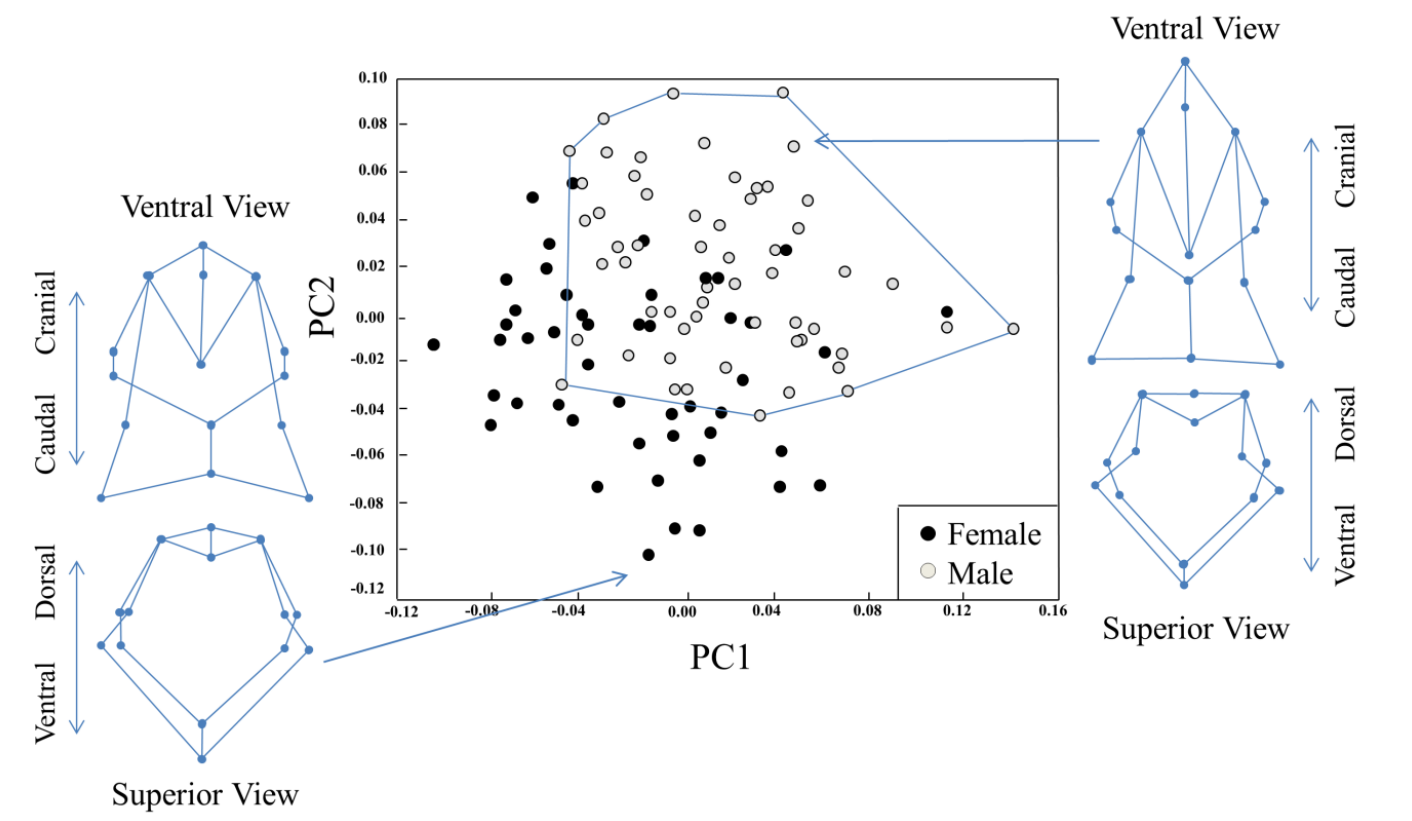

Figure 2.10a: Principal components analysis of birth canal shape in Gorilla gorilla. Note the diverging distributions of males and females along Axis 1 and Axis 2.

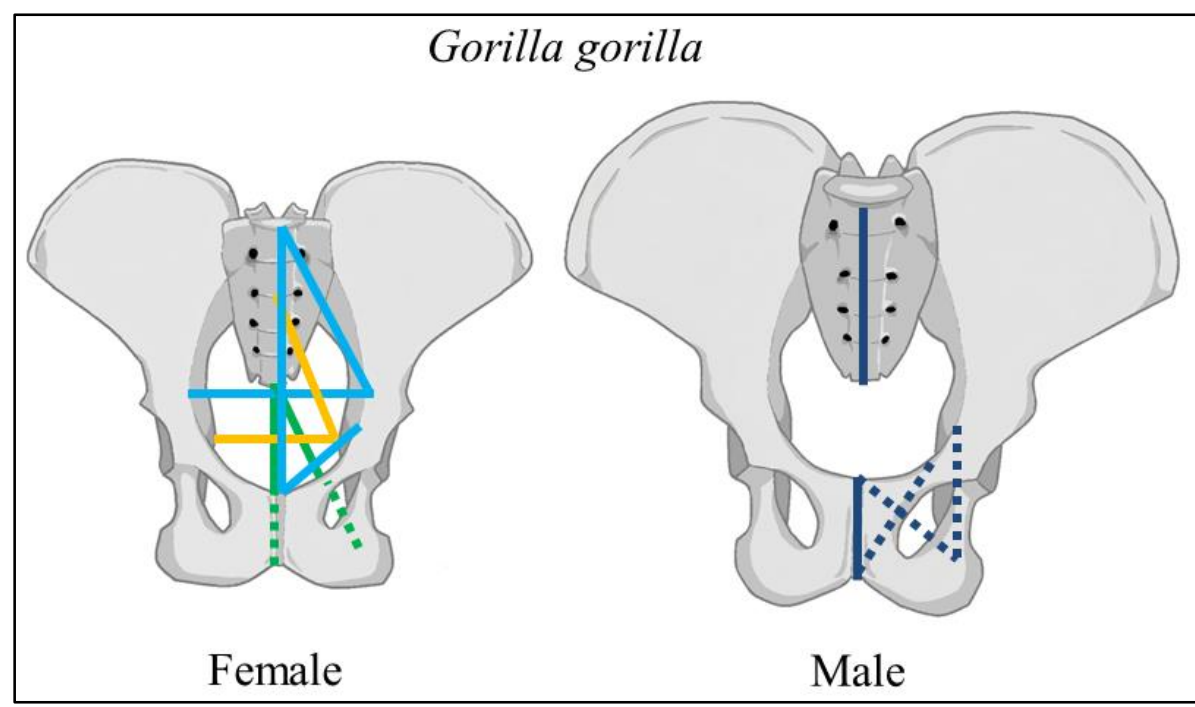

yellow represents distances greater in females in the midplane, and green represents distances greater in females in the outlet. Dark blue represents linear distances relatively greater in males. Only non-overlapping distances are included.
Figure 2.10b: Results of FORM procedure to obtain dimorphic distances in the pelvis of Gorilla gorilla when pelves are scaled to geometric mean. Light blue represents distances greater in females than males in the inlet, 


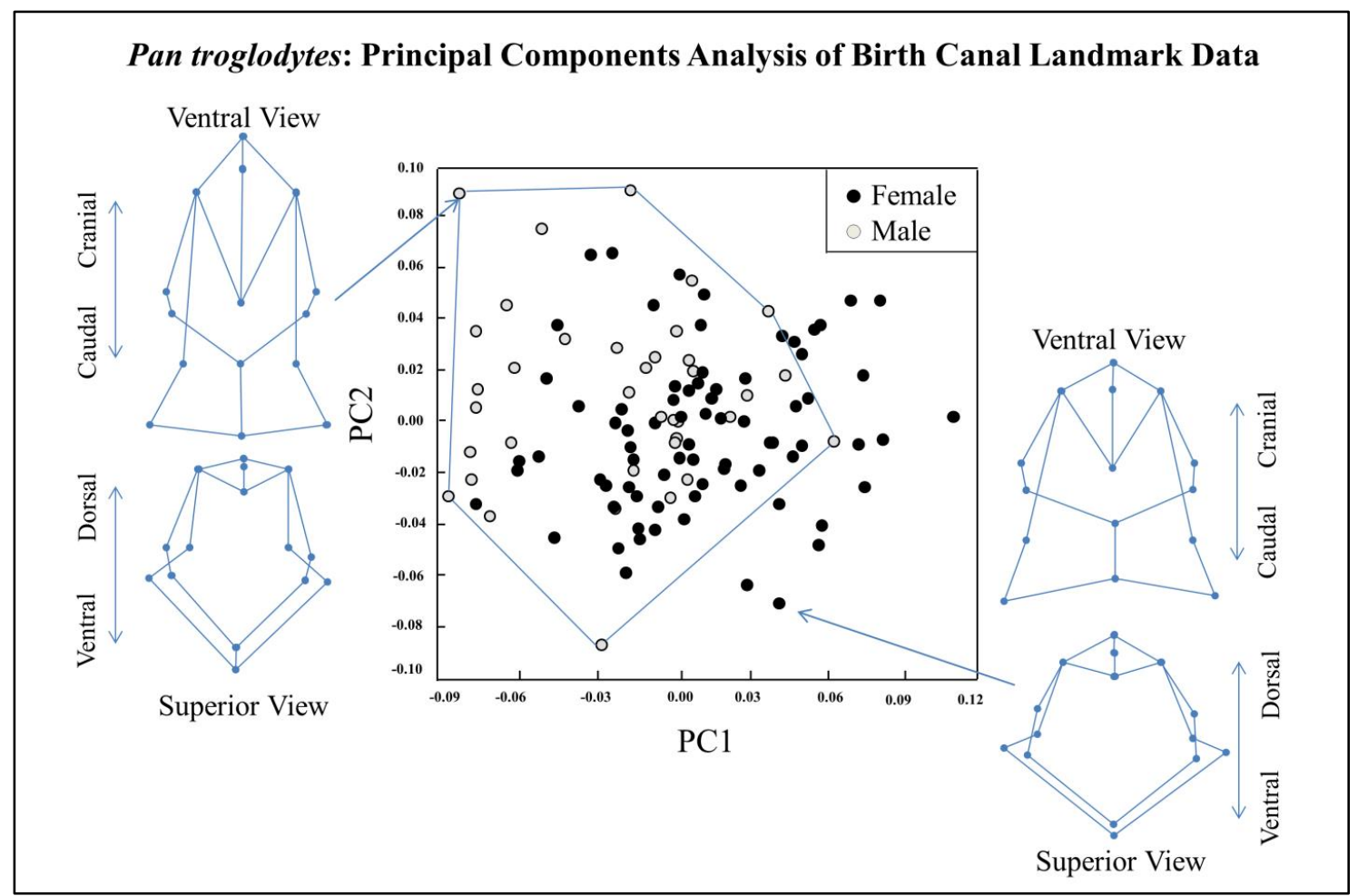

Figure 2.11a: Principal components analysis of birth canal shape in Pan troglodytes. Note lack of diverging distributions of males and females.

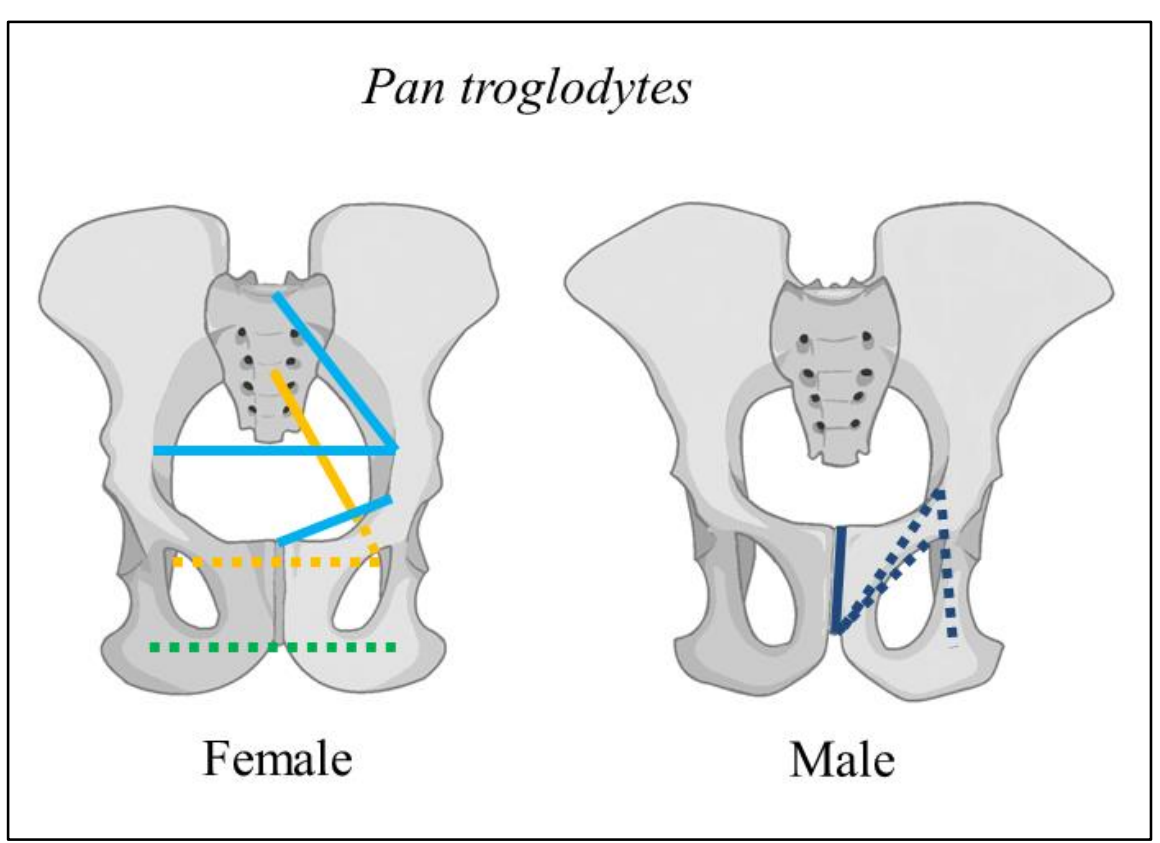

Figure 2.11b:

Results of FORM procedure in Pan troglodytes when pelves are scaled to geometric mean. Light blue represents distances greater in females than males in the inlet, yellow represents distances greater in females in the midplane, and green represents distances greater in females in the outlet. Dark blue represents distances relatively greater in males. Only non-overlapping distances are included. 


\section{Alouatta seniculus: Principal Components Analysis of Birth Canal Landmark Data}

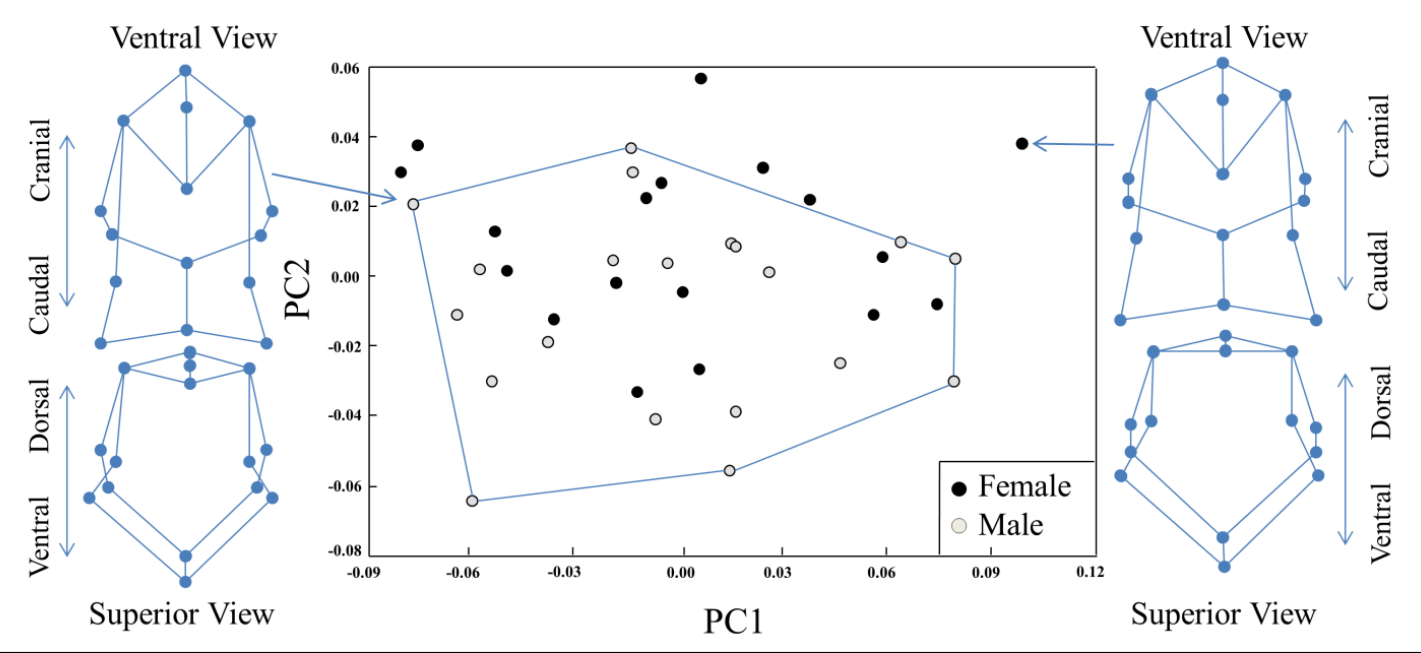

Figure 2.12a: Principal components analysis of birth canal shape in Alouatta seniculus. Note lack of diverging distributions of males and females.

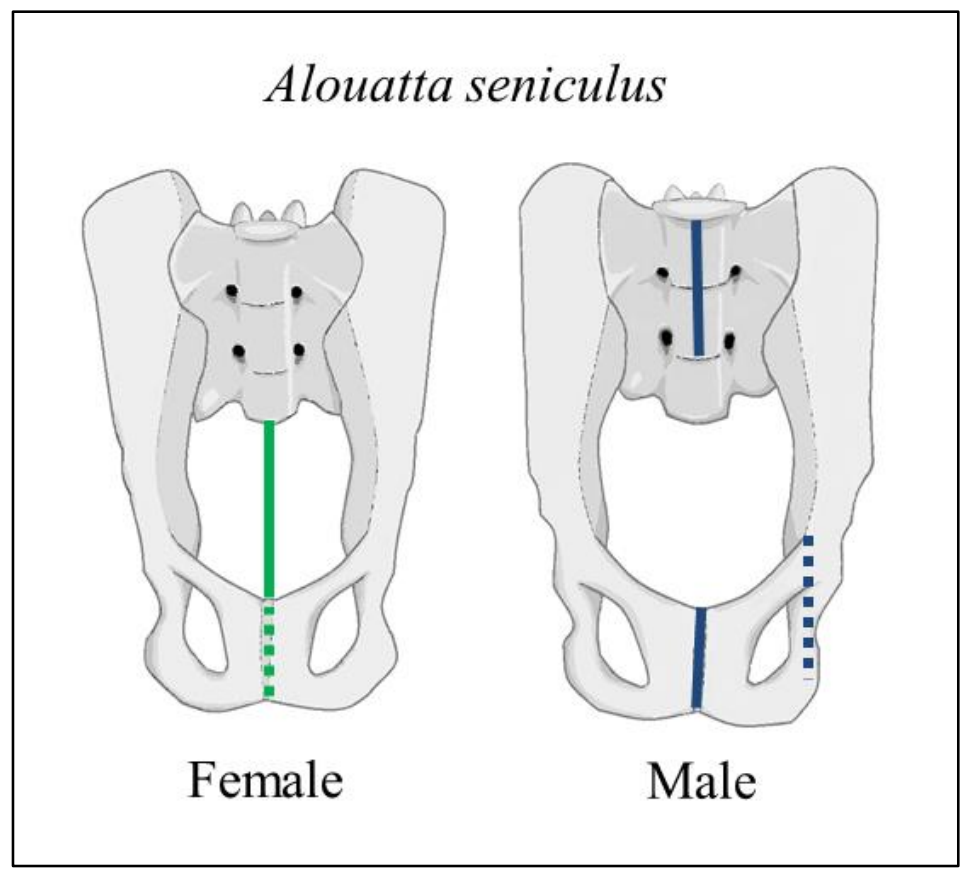

Figure 2.12b: Results of FORM procedure in Alouatta seniculus when pelves are scaled to geometric mean. Light blue represents distances greater in females than males in the inlet, yellow represents distances greater in females in the midplane, and green represents distances greater in females in the outlet. Dark blue represents distances relatively greater in males. Only non-overlapping distances are included. 


\section{CHAPTER 3: DIMORPHISM IN RELATIVE SACRAL BREADTH AMONG CATARRHINE PRIMATES}

\section{BACKGROUND}

Chapter 2 of this study finds that the magnitude of dimorphism in birth canal size and shape is generally greater among species with large cephalopelvic proportions compared to species with small cephalopelvic proportions, leading to the hypothesis that the greater magnitude of dimorphism in the birth canals of species with large cephalopelvic proportions may be due to the presence of dimorphism in the breadth of the sacrum among taxa with large proportions. The sacrum contributes to the size and shape of the inlet, midplane, and outlet of the bony pelvis and thus represents a potentially important target for obstetric selection. However, very few studies of sacral sexual dimorphism in non-human primates have been conducted (Tague, 1991; Tague, 1992). Consequently, little is currently known about either the existence of sexual dimorphism in the sacrum of extant non-human primates, or what factors may lead to dimorphic sacral morphology, if present. Moreover, such a limited understanding of primate sacral sexual dimorphism constrains interpretation of fossil hominin sacral morphology (Robinson, 1972; Leutenegger, 1977; Berge et al., 1984; Rosenberg, 1986; Tague and Lovejoy, 1986; Tague, 1991). This is unfortunate given the number of wellpreserved sacra in the hominin fossil record, including Stw 431 and Sts 14 (Australopithecus africanus), A.L. 288-1 (A. afarensis), MH 2 (A. sediba), BSN49/P27 
(Homo erectus), SH 1 (H. heidelbergensis) and the Kebara 2 Neandertal (Leutenegger, 1977; Johanson et al., 1982; Rak and Arensburg, 1987; Arsuaga et al., 1999; Toussaint et al., 2003; Simpson et al., 2008; Bonmatí et al., 2010; Kibii et al., 2011).

\section{a. Dimorphism in the human sacrum}

Hominin sacral morphology is often interpreted in light of both locomotor and obstetric adaptations (Waterman, 1929; Robinson, 1972; Abitbol, 1987a; Ward, 2002) due to the fact that humans are both bipedal and have notoriously large cephalopelvic proportions (large size of the neonate head compared to the dimensions of the pelvic planes). Accordingly, both males and females in the genus Homo are widely argued to be under selection for reduced dimensions of the pelvic planes in order to facilitate efficient bipedal locomotion (Washburn, 1950, 1960; Pinkerton, 1973; McHenry, 1975; Zihlman, 1978). However, human females, unlike their male counterparts, are believed to be simultaneously under selection to maintain an obstetrically sufficient bony pelvis, and it is this "obstetric dilemma" in females which has typically been viewed as a primary factor driving dimorphic pelvic morphology in humans (Berge et al., 1984; Tague and Lovejoy, 1986; Abitbol, 1987b; Hager, 1989; Rosenberg, 1992; Abitbol, 1996; Rosenberg and Trevathan, 2002; but see Dunsworth et al., 2012; Wells et al., 2012). Although it is currently unclear at what point during hominin evolution neonatal encephalization initiated increased cephalopelvic proportions, it has previously been suggested that cephalopelvic proportions similar to modern humans may have evolved as early as Homo erectus (Walker and Ruff, 1993; Ruff, 1995; Simpson et al., 2008; but see DeSilva, 2011) and were almost certainly present among H. heidelbergensis, 
Neandertals, and early H. sapiens (Arsuaga et al., 1999; Ponce de León et al., 2008; Franciscus, 2009; Weaver and Hublin, 2009; see also Rosenberg et al., 2006).

Given the important evolutionary implications of pelvic morphology, it is perhaps unsurprising that sexual dimorphism in the human sacrum has long garnered anthropological interest, as the first sacral (S1) vertebra comprises the posterior border of the pelvic inlet (Paterson, 1893; Derry, 1912; Trotter, 1926; Fawcett, 1938; Flander, 1978; Kimura, 1982; Tague, 2007; Benazzi et al., 2009). Interestingly, however, it is not overall width of the sacrum which exhibits sexual dimorphism in humans (Flander, 1978; Tague, 1992). Rather, it is the relative contributions to the overall width of the sacrum by the alae and $\mathrm{S} 1$ vertebral body that exhibits sexual dimorphism in humans, with the alae constituting a greater proportion of overall breadth in females compared to males (Fawcett, 1938; Flander, 1978; Tague, 2007; Benazzi et al., 2009). Indeed, breadth of the $\mathrm{S} 1$ alae is one of the most highly dimorphic measurements of the human pelvis (Tague, 1992; 2007). Breadth of the S1 alae is also positively correlated with pelvic inlet breadth (Tague, 2007), suggesting the relative lateral expansion of the sacral alae among human females likely reflects functional differences between male and female pelves (see also Flander and Corruccini, 1980), with expanded sacral alae in females likely a response to heightened obstetric demands (Tague, 2007).

\section{b. Dimorphism in the sacra of other primates}

It remains unexplored whether any anthropoid taxa besides humans exhibit dimorphism in sacral breadth controlling for body size, but it is possible that obstetric 
demand influences dimorphism in relative sacral breadth in non-human primates, as obstetric demand is thought to result in the sexually dimorphic sacra of humans (Fawcett, 1938; Flander, 1978; Tague, 2007; Benazzi et al., 2009). Humans are not the only primates with large cephalopelvic proportions (Table 1; Schultz, 1949; Stoller 1995), and it is possible that these other primate species with large cephalopelvic proportions also exhibit dimorphism in the breadth of the sacral alae (with females exhibiting wider sacra than males controlling for overall body size). If obstetric demand is the only factor which influences dimorphism in the breadth of the sacrum, it would predicted that species with small cephalopelvic proportions are not dimorphic in sacral breadth.

However, obstetric demand is not the only factor which could lead to dimorphism in sacral breadth. While many researchers relate dimorphism in features such as the ischo-pubic index of primates primarily to cephalopelvic proportions (Leutenegger, 1974; Mobb and Wood, 1977; Wood and Chamberlain, 1986; Ridley, 1995), others link pelvic dimorphism in primates chiefly to overall body size dimorphism (Steudel, 1981, 1984; Tague, 2005). Pelvic dimorphism may be greater among species that are highly dimorphic in body size compared to species that are monomorphic in body size (Schultz, 1949; Tague, 1991; 2005; see also Chapter 2), and this could indicate a non-isometric relationship between pelvic shape and body size within highly dimorphic species. Any disparities between male and female pelvic morphology could be due to allometry (Steudel, 1981) biomechanical differences between the sexes (Steudel, 1981, 1984) or hormonally-driven modification of the male pelvis beyond the "default" morphology of the female (Tague, 2005). 
Finally, it is possible that the presence of dimorphism in sacral breadth in anthropoids is due to a complex interplay of body size dimorphism, obstetric demand, and phylogeny (Schultz, 1949; Tague, 1991; 2005). Schultz (1949) specifically relates pelvic sexual dimorphism among monkeys and humans to obstetric factors, but argues body size dimorphism to be the primary influence on pelvic dimorphism among the other apes, including gibbons (which have large cephalopelvic proportions). Similarly, Chapter 2 of the current study finds that dimorphism in the size and shape of the birth canal exists across anthropoid taxa, but that the magnitude of dimorphism in birth canal size and shape is greatest among species with both large cephalopelvic proportions and high levels of body size dimorphism. Clearly, both cephalopelvic proportions and body size dimorphism influence dimorphism in the birth canals of primates (Schultz, 1949; Leutenegger, 1974), and it is possible that both of these factors can lead to dimorphism in sacral breadth in anthropoid taxa.

Although sacral breadth relative to $\mathrm{S} 1$ vertebral body breadth is known to be dimorphic in humans (Fawcett, 1938; Flander, 1978; Tague, 2007), to the authors' best knowledge, no research has been conducted examining dimorphism in this ratio among non-human primates. Therefore, an inter-specific evaluation of dimorphism in this ratio would clarify the relationships among pelvic dimorphism, relative neonate size, and body size dimorphism in primates.

Specifically, the existence of sexual dimorphism in the relative breadth of the human sacrum leads to two important questions about sexual dimorphism of the sacrum in non-human primates: 
1) Is sexual dimorphism in breadth of the sacrum relative to $S 1$ vertebral body breadth present in non-human primates?

2) If present in non-human primates, is sexual dimorphism in this ratio related specifically to obstetrics, body size dimorphism, or a combination of these factors?

The purpose of the present study is first to determine whether sexual dimorphism of the sacrum exists in other anthropoid species, and second, to test hypotheses related to obstetrics, body size dimorphism, and relative sacral breadth in humans and non-human primates.

\section{MATERIALS AND METHODS}

\section{a. Samples}

For this study, measurements of lumbar and sacral vertebrae were collected on a total of 266 primate specimens, encompassing Homo sapiens $(\mathrm{n}=71)$, Hylobates lar $(\mathrm{n}=69)$, Gorilla gorilla $(\mathrm{n}=44)$, Pan troglodytes $(\mathrm{n}=27)$, Nasalis larvatus $(\mathrm{n}=27)$, Pongo pygmaeus $(\mathrm{n}=15)$, and Pan paniscus $(\mathrm{n}=13)$. This sample differs from the sample from Chapter 1 because it was necessary to take measurements on lumbar vertebrae for this study (see below). All specimens of non-human primates were obtained from wild populations. In order to ensure that only adult specimens were included, measurements were only taken on specimens in which the apophyses of the iliac crest and ischial tuberosity are fused. Furthermore, all specimens were free of obvious pathology, and only specimens with modal numbers of lumbar and sacral vertebrae (Schultz and Straus, 
1945) were included. Sampled taxa were chosen to encompass a wide spectrum of body size dimorphism known to exist in living primates as well as a range of relative neonate head dimensions to adult female pelvic inlet size combinations (Table 3.1; Figure 3.1). This sample consists primarily of hominoid taxa, with Nasalis larvatus representing the only non-hominoid species. While the inclusion of a cercopithecine species in an otherwise hominoid-only sample presents an obvious phylogenetic confound, the inclusion of $N$. larvatus was integral to determining the influence of cephalopelvic proportions and body size dimorphism within primates, as this species is characterized by both large body size dimorphism and large cephalopelvic proportions, a combination which does not exist among living hominoids. Moreover, inclusion of this species also allowed comparisons of sacral sexual dimorphism between hominoids and nonhominoids.

Specimens of Hylobates lar, Pongo pygmaeus, Gorilla gorilla, and Pan troglodytes, as well as specimens of Homo sapiens at the University of Iowa-Stanford Collection (all collected by Elizabeth A. Moffett) were inlcuded. Specimens of Pan paniscus collected at the Royal Museum of Central Africa, additional specimens of $H$. lar at the Museum of Comparative Zoology; G. gorilla, P. pygmaeus, and P. troglodytes at the Cleveland Museum of Natural History; and G. gorilla, N. larvatus, P. pygmaeus, and P. troglodytes at American Museum of Natural History (all collected by Carol V. Ward; Table 3.1) were also included. Sample size was determined as the maximum number of possible individuals of each species at these institutions. Interobserver error for each measurement was assessed using technical error of measurement (Perini et al., 2005) as described by Moffett et al. (2013) on one specimen of Homo sapiens and one 
specimen of Pan troglodytes. For the H. sapiens specimen, the absolute and relative (in parentheses) technical errors were as follows: lumbar vertebral body breadth $=0.383$ $(0.8 \%)$, lumbar bi-transverse breadth $=0.138(0.2 \%), \mathrm{S} 1$ vertebral body breadth $=0.594$ $(1.4 \%)$, and sacral breadth at linea terminalis $=0.981(1.1 \%)$. For the $P$. troglodytes specimen, the absolute and relative (in parentheses) technical errors of measurement for lumbar vertebral body breadth, lumbar bi-transverse breadth, S1 vertebral body breadth, and sacral breadth at linea terminalis were $0.071(0.2 \%), 0.263(0.4 \%), 0.167(0.4 \%)$, and $0.501(0.8 \%)$ respectively. The low values for intraobserver technical error indicate a high level of repeatability for each observer.

Finally, sampled taxa were divided into four categories based on distinct combinations of body size dimorphism and cephalopelvic proportions (summarized in Table 3.1). The assignment of taxa to specific cephalopelvic categories was based on previous studies of cephalopelvic proportions in the taxa included here (Schultz, 1949; Smith and Jungers, 1997). While an obvious method for assigning taxa to body size dimorphism categories would be to simply employ body mass dimorphism, previous authors (Gordon, 2006; Plavcan, 2012) have noted that there is not a uniform correspondence between body mass and skeletal dimorphism. Accordingly, categorization based on body size dimorphism was based on both previously published values for body mass dimorphism (Smith and Jungers, 1992) and one indicator of skeletal dimorphism (Table 3.1; Figure 3.1a), namely the index of dimorphism in caudal-most lumbar vertebral body width, as size of the caudal-most lumbar vertebral body is known to be highly correlated with body mass in anthropoid primates (Nakatsukasa and Hirose, 2003). This lumbar dimorphism index based on linear 
measurements was cubed [(male mean lumbar vertebral body breadth/female mean

lumbar vertebral body breadth $)^{3}$ ] to permit direct comparisons with the body mass dimorphism index. Based on these cephalopelvic proportion and body size dimorphism data (Figure 3.1b), each species was assigned to one of the four following categories:

\section{Category A (minimal to moderate body size dimorphism and large} cephalopelvic proportions): This category is represented by two species, Homo sapiens and Hylobates lar. Both species exhibit low levels of body size dimorphism (i.e., have a male/female body mass index falling between 1.001.30) such that they are generally considered to be either monomorphic (H. lar) or minimally dimorphic (H. sapiens) in body size (Smith and Jungers, 1997). The lumbar vertebral body breadth dimorphism index also indicates that these species are minimally skeletally dimorphic (1.06 in $H$. lar and 1.23 in $H$. sapiens). Moreover, these two species have large cephalopelvic proportions with neonates that occupy over $90 \%$ of the transverse diameter of the pelvic inlet at the time of birth (Schultz, 1949).

\section{Category B (minimal to moderate body size dimorphism and small} cephalopelvic proportions): This category encompasses the species Pan troglodytes and Pan paniscus. These two species exhibit both low body size dimorphism (male/female body mass index 1.30 or lower), a low index of lumbar vertebral body breadth dimorphism (1.12 and 1.00, respectively) and relatively small neonate size, with the neonatal head reaching only $72 \%$ of the transverse diameter of the maternal pelvic inlet at term (Schultz, 1949; Smith and Jungers, 1997). 


\section{Category C (large body size dimorphism and large cephalopelvic}

proportions): This category is composed of a single species: Nasalis larvatus.

This species has extremely high levels of body size dimorphism, with adult males having over twice the body mass of females (Smith and Jungers, 1997), and the lumbar vertebral body dimorphism index of this species (1.87) indicates that males are substantially larger than females for this skeletal measure. Additionally, this species gives birth to relatively large neonates, with the breadth of the full-term neonate head occupying $95 \%$ the diameter of the maternal pelvic inlet (Schultz, 1949).

\section{Category D (large body size dimorphism and small cephalopelvic}

proportions): Category D is represented by two species, Gorilla gorilla and Pongo pygmaeus. These species are characterized by high levels of body size dimorphism in which adult male body mass is typically over twice that of adult females (Smith and Jungers, 1997). The lumbar vertebral body breadth index further indicates that $P$. pygmaeus and $G$. gorilla are highly dimorphic, with index values of 1.69 and 1.91, respectively. Moreover, these species give birth to relatively small neonates, with the transverse diameter of the neonate head at term reaching less than $75 \%$ the diameter of the pelvic inlet (Schultz, 1949).

\section{b. Methods}

Two measurements of the sacrum (Figure 3.2) were collected on each specimen: 1) S1 vertebral body width and 2) sacral breadth at linea terminalis. Most previous 
studies (e.g., (Fawcett, 1938; Flander, 1978; Tague, 2007) have measured maximum breadth of the sacrum (i.e., the widest distance across the sacral alae). However, in the present study, breadth of the sacrum was specifically measured at linea terminalis because this measurement quantifies the contribution of the sacrum to the posterior portion of the pelvic inlet and is thus a more obstetrically-relevant measurement. In order to collect sacral breadth at linea terminalis, the sacrum and os coxae were articulated using the method described by Tague $(1992 ; 2007)$, and the linear distance was taken between the sacroiliac joints on the anterior sacrum at the level of linea terminalis (Figure 3.2a).

In order to evaluate whether potential sexual dimorphism in the sacrum simply reflects a more systematic expression of body size dimorphism in primates, two measurements of the caudal-most lumbar vertebrae were also collected on each specimen (Figure 3.2b): lumbar vertebral body width and bi-transverse process breadth. The caudal-most lumbar vertebra was specifically chosen because this vertebra has previously been shown to be highly correlated with body size in primates (Nakatsukasa and Hirose, 2003), and permits similar measurements to those taken on the sacrum (i.e., overall and vertebral body breadths) to be assessed on an adjacent vertebral element that is not directly affected by obstetric demands (although lumbar bi-transverse breadth and sacral breadth at linea terminalis are likely functionally integrated for non-obstetric reasons).

Absolute measures of sacral or lumbar vertebrae are of limited utility for evaluating sexual dimorphism, however, as absolute measures of sacral breadth at linea terminalis or bi-transverse process breadth of the lumbar vertebra are expected to be 
sexually dimorphic in species in which males have much larger body size than females. However, the fact that males have absolutely wider measurements in such species does not preclude the possibility of females possessing relatively wider sacra or lumbar vertebra when controlled for vertebral body size, which is the question being investigated here. Thus, two ratios were computed:

(1) Relative Sacral Breadth $=\frac{\text { sacral breadth at linea terminalis }}{\text { S1 vertebral body width }}$

(2) Relative Lumbar Breadth $=\frac{\text { lumbar bi-transverse breadth }}{\text { lumbar vertebral body width }}$

In these ratios, the overall breadth of each vertebra (i.e., breadth of the sacrum at linea terminalis or bi-transverse process breadth of the lumbar vertebra) was divided by the breadth of the corresponding vertebral body width, and the values thus represent the relative contribution of the alae or transverse processes to the overall breadth of the vertebral element. Because all relationships investigated here were isometric (see below), these ratios create a measure of relative vertebral breadth that was independent of the absolute size of the vertebral element, allowing comparisons within species that have large sexual body size dimorphism (e.g., Pongo pygmaeus) and between species that are largely divergent in body size (e.g., Gorilla gorilla versus Hylobates lar).

In order to verify isometric scaling in the ratios employed in the study, natural log-tranformed values of lumbar bi-transverse process breadth and sacral breadth at linea terminalis were regressed on their respective natural log-transformed vertebral body widths using reduced major axis regression (Table 3.2). Slope values for sacral and lumbar regressions across all species both showed some deviation from isometry, with the sacrum exhibiting slight positive allometry (slope $=1.111,95 \%$ Confidence 
Interval $=1.072-1.150)$ and lumbar vertebrae exhibiting slight negative allometry (slope $=0.939,95 \% \mathrm{CI}=0.897-0.981)$. However, in both cases these deviations could be attributed to the undue influence of specific outlying species rather than a consistent allometric trend across primates. For the sacrum, it is apparent that humans, which exhibit exceptionally wide sacral alae compared to other primate species (Abitbol, 1987a; Lovejoy, 2005), are driving the allometric result, as removal of this species results in an isometric slope (slope $=0.984, \mathrm{CI}=0.943-1.024$ ) across all other primate species. Conversely, allometry in the lumbar vertebrae is clearly driven by the morphology of the only cercopithecine and palmigrade quadruped in the sample, Nasalis larvatus, with isometry characterizing all other species (slope $=0.969, \mathrm{CI}=$ 0.928-1.010). These results suggest that structural proportions between the vertebral body and alae/costal processes are generally maintained in primates regardless of size, indicating that the use of natural $\log$-transformed ratios in this study is appropriate for species-specific between-sex comparisons.

Due to unequal sample sizes between sexes within species, and potential issues regarding homogeneity of variances between species, randomization tests on natural log-transformed ratios were carried out on a per-species basis to test for differences in relative sacral and lumbar breadths between males and females in each species. Ratios are natural log-transformed because ratios frequently exhibit non-normal distributions (Atchley et al., 1976). Randomization tests are permutation tests which identify how likely the obtained group differences could be obtained by chance (Good, 1994; Edgington, 1995), and are commonly used when examining differences between groups with small sample sizes, as sample size is irrelevant to the validity of the test statistic 
(Ninness et al., 2002). For each test, the p-value was determined analytically via randomization using 100,000 Monte Carlo simulations (Roff, 2006), with a significance level of $\alpha=0.05$. Subsequently, the four previously described cephalopelvic proportions to body size dimorphism categories were employed to test a set of four specific hypotheses regarding sexual dimorphism in relative sacral breadth among primates (Table 3.3).

Hypothesis 1: Sexual dimorphism in relative sacral breadth does not exist in nonhuman catarrhines.

Prediction 1a: This hypothesis predicts that no species included in this study should exhibit dimorphism in relative sacral breadth.

Hypothesis 2: Sexual dimorphism in relative sacral breadth is exclusively due to body size dimorphism.

Predition 2a: This hypothesis postulates that dimorphism in relative sacral breadth should exist in both Categories C and D (large body size dimorphism) but should not exist in Categories A and B (minimal body size dimorphism).

Hypothesis 3: Sexual dimorphism in relative sacral breadth is exclusively related to cephalopelvic proportions.

Prediction 3a: This hypothesis anticipates that dimorphism in relative sacral breadth should exist in both Categories A and C (relatively large neonates), but should not exist in Categories B and D (relatively small neonates). 
Hypothesis 4: Sexual dimorphism in relative sacral breadth is not exclusively due to either body size dimorphism level or cephalopelvic proportions.

Prediction 4a: This hypothesis posits that sexual dimorphism in relative sacral breadth cannot be conclusively related to just body size dimorphism level or cephalopelvic proportions (see above). Accordingly, this hypothesis would suggest that sexual dimorphism in relative sacral breadth is related to either a combination of both body size dimorphism level and cephalopelvic proportions, and/or phylogeny.

Criteria used to reject each hypothesis are summarized in Table 3.3. In order to test Hypothesis 1 (lack of dimorphism in relative sacral breadth in non-human species), distributions of male and female relative sacral breadth values are first evaluated for statistical differences in each species. The presence of statistically significant sexual dimorphism in one or more of the six non-human species was taken as grounds for rejection of Hypothesis 1, which was subsequently followed by simultaneous testing of Hypothesis 2 and Hypothesis 3. Rejection of Hypothesis 2 (dimorphism in relative sacral breadth exclusively due to body size dimorphism) requires that statistically significant levels of sexual dimorphism in relative sacral breadth be found in any species in Categories A or B (minimal body size dimorphism). Grounds for rejection of Hypothesis 3 (dimorphism in relative sacral breadth exclusively due to cephalopelvic proportions) included the presence of statistically significant levels of dimorphism in relative sacral breadth in any species in Categories B or D (relatively small neonates). Rejection of both Hypothesis 2 and Hypothesis 3 would result in the adoption of Hypothesis 4 (dimorphism in relative sacral breadth due to a combination of body size 
dimorphism and cephalopelvic proportions and/or to phylogeny) as a null hypothesis for future research.

Finally, to determine whether potential dimorphism in relative sacral breadth is due to differences in sacral breadth at linea terminalis relative to $\mathrm{S} 1$ vertebral breadth or vice versa, the absolute breadth of the $\mathrm{S} 1$ vertebral body and sacral breadth at linea terminalis were evaluated for sexual dimorphism using randomization tests of differences in means. Specifically, among any species found to exhibit sexual dimorphism in relative sacral breadth, the relationship between S1 vertebral breadth and sacral breadth at linea terminalis was examined to determine if all sexually dimorphic species shared a common morphological pattern or whether dimorphism could be attributed to multiple sacral morphologies. Again, due to sample size considerations, the p-values were determined via randomization using 100,000 Monte Carlo simulations.

\section{RESULTS}

Results for this study are summarized in Tables 3.4 and 3.5. Samples in Category A, encompassing Hylobates lar $(\mathrm{p}<0.001)$ and H. sapiens $(\mathrm{p}<0.001)$; Category $\mathrm{C}$, including the single species $N$. larvatus $(\mathrm{p}=0.019)$; and a single species in Category D, Gorilla gorilla $(\mathrm{p}<0.001)$, are found to have statistically significant levels of sexual dimorphism in relative sacral breadth (Table 3.5). Due to the fact that sexual dimorphism in relative sacral breadth is identified in multiple primate species in this study, Hypothesis 1 (non-existence of sexual dimorphism in relative sacral breadth) is rejected. 
Because sexual dimorphism in relative sacral breadth is found to exist in minimally dimorphic species (Category A), predictions based upon Hypothesis 2 (dimorphism in relative sacral breadth exclusively related to body size dimorphism) are not substantiated, and this hypothesis was accordingly rejected. Additionally, Hypothesis 3 (dimorphism in relative sacral breadth related exclusively to relative neonate size) is rejected, as Gorilla gorilla, which has small cephalopelvic proportions, is also found to be dimorphic for relative sacral breadth. Moreover, in all species found to be dimorphic for relative sacral breadth, including gorillas, females are found to have statistically larger relative sacral breadth values than males (Table 3.5). As sexual dimorphism in relative sacral breadth is not found to be related exclusively to body size dimorphism (Hypothesis 2) or cephalopelvic proportions (Hypothesis 3) in this study, Hypothesis 4 (sexual dimorphism in relative sacral breadth is not exclusively due to either body size dimorphism level or cephalopelvic proportions) cannot be rejected.

Nasalis larvatus is the only species with statistically significant levels of sexual dimorphism in both relative sacral breadth and relative lumbar breadth $(\mathrm{p}=0.033)$. In contrast, the other three species found to be dimorphic for relative sacral breadth (Homo sapiens, Hylobates lar, and Gorilla gorilla) are not dimorphic for relative lumbar breadth $(\mathrm{p}=0.695, \mathrm{p}=0.290$, and $\mathrm{p}=0.588$ respectively).

For the four species dimorphic for relative sacral breadth, dimorphism in absolute breadth of S1 and sacral breadth at linea terminalis is further evaluated (Table 3.4). Homo sapiens is found not to be dimorphic for sacral breadth at linea terminalis but is dimorphic for S1vertebral body width $(\mathrm{p}<0.001)$ with males possessing significantly larger S1 vertebral bodies than females. In contrast, Hylobates lar is not 
found to be dimorphic for S1 vertebral body width but is significantly sexually dimorphic for sacral breadth at linea terminalis $(\mathrm{p}<0.001)$, with female gibbons being larger than males for this variable. Nasalis larvatus is significantly dimorphic for both breadth of $\mathrm{S} 1(\mathrm{p}<0.001)$ and sacral breadth at linea terminalis $(\mathrm{p}<0.001)$ with males absolutely larger than females in both measurements. Similarly, Gorilla gorilla is also significantly dimorphic for both breadth of $\mathrm{S} 1(\mathrm{p}<0.001)$ and sacral breadth at linea terminalis $(\mathrm{p}=0.001)$.

\section{DISCUSSION AND CONCLUSIONS}

In this study, four species - Homo sapiens, Hylobates lar, Nasalis larvatus and Gorilla gorilla - are found to be sexually dimorphic in relative sacral breadth (Figure 3.3). These four species exhibit variability in overall body size dimorphism ranging from monomorphic to highly dimorphic. Of these, $H$. sapiens, $H$. lar, and $N$. larvatus have large cephalopelvic proportions, whereas G. gorilla has small cephalopelvic proportions. In contrast, Pongo pygmaeus, Pan troglodytes, and Pan paniscus, are not statistically sexually dimorphic for relative sacral breadth. The results of this study therefore indicate that sexual dimorphism in relative sacral breadth does indeed exist in some species of non-human primates, and that, at least among catarrhines, dimorphism in relative sacral breadth is not exclusively related to either cephalopelvic proportions or to body size dimorphism. However, because all of the species with large cephalopelvic proportions included in this study (H. sapiens, H. lar, and $N$. larvatus) exhibit dimorphism in relative sacral breadth, the results of this study do broadly suggest that, 
among species with large cephalopelvic proportions, there is selective pressure on females to possess relatively broader sacral alae than their male counterparts.

A more detailed inspection of the data reveals that the relationship between overall sacral breadth, S1 vertebral body breadth, and length of the alae varies considerably among Homo sapiens, Hylobates lar, Nasalis larvatus, and Gorilla gorilla. Results indicate that sacral breadth at linea terminalis is not sexually dimorphic in $H$. sapiens, whereas breadth of the $\mathrm{S} 1$ vertebral body is significantly dimorphic, with human females possessing absolutely narrower vertebral bodies than males. These finding are in accordance with previous research (Fawcett, 1938; Flander, 1978; Tague, 2007; Benazzi et al., 2009), and since these results show that human females have narrower S1 vertebral bodies, but similar overall sacral widths, it is clear that relatively wide sacral alae in human females serves to produce a disproportionally wide sacrum relative to a small vertebral body. As S1 vertebral body size closely tracks overall body size in humans (McHenry, 1992), females among H. sapiens possess relatively, but not absolutely, wider sacra than males when body size is taken into account. Conversely, $H$. lar is significantly dimorphic for sacral breadth at linea terminalis, but not S1 vertebral body breadth. Thus, for gibbons, elongated sacral alae actually produce an absolutely wider sacral breadth in females compared to males, despite the similarity in vertebral body size between the sexes (Figure 3.3). Consequently, while some authors (Schultz, 1949; Steudel, 1981; Tague, 2005) have argued the pelvis of $H$. lar to be monomorphic, these results support the findings of Leutenegger (1974) and Ridley (1995) who found features of the gibbon pelvis, such as the ischio-pubic index, to be moderately dimorphic. 
The remaining two dimorphic species, Nasalis larvatus and Gorilla gorilla, are each found to be sexually dimorphic for both sacral breadth at linea terminalis and S1 vertebral body breadth, with males exhibiting absolutely larger values for both measurements (Table 3.4). However, comparisons of the relative magnitude of differences in the two measurements indicate that, despite their larger absolute size, males of both species exhibit disproportionally narrower alae relative to their vertebral bodies when compared to those of females. As a result, male $N$. larvatus possess sacra that are, on average, only $14 \%$ wider than those of females, despite possessing S1 vertebral bodies $20 \%$ larger than their female counterparts. This trend is even more pronounced among G. gorilla, in which males have S1 vertebral bodies $21 \%$ larger than those of females but sacra that are on average only $7 \%$ wider than females.

The finding of sexual dimorphism in relative sacral breadth in Gorilla gorilla is particularly interesting in the context of this study, as, opposed to Homo sapiens, Hylobates lar, and Nasalis larvatus, this species represents the only taxon in which dimorphism in relative sacral breadth was found in conjunction with small cephalopelvic proportions. Thus, given these small cephalopelvic proportions, it appears unlikely that dimorphism in relative sacral breadth in G. gorilla is related to obstetric demands placed on females. Consequently, this raises the possibility that sacral dimorphism among gorillas may be primarily attributable to selection on males for relatively narrower sacra, rather than relatively broader sacra in females. While it is possible that potential selection on relative sacral breadth among G. gorilla males could be related to large body size dimorphism alone, this appears unlikely considering that Pongo pygmaeus, which is also characterized by large male body size, does not exhibit 
dimorphism in relative sacral breadth. Alternatively, previous authors have related both narrower sacra (Abitbol, 1987a; Lovejoy, 2005) and greater lumbar entrapment (Lovejoy et al., 2009a; Lovejoy et al., 2009b) in apes compared to hominins to locomotor demands. Accordingly, relatively narrow sacra in gorilla males may be related to unique locomotor pressures faced by large male gorillas during terrestrial knuckle-walking (compared to smaller female gorillas and chimpanzees), or to decreased frequency of suspensory postures among G. gorilla males compared to females (Remis, 1995). Thus, the results of the current study highlight the need to more fully understand selection on male pelvic morphology, especially among species in which males and females are largely divergent in body size and/or exhibit slight differences in locomotor patterns.

In contrast to gorillas, in which sexual dimorphism in relative sacral breadth appears predominantly related to body size dimorphism, in Nasalis larvatus, dimorphism in relative sacral breadth is found in association with both large body size dimorphism and large cephalopelvic proportions. Thus, while the presence of large cephalopelvic dimensions in $N$. larvatus suggests at least one evolutionary pressure exerted on female sacral morphology (especially given the demonstrated changes to female sacra in other primate species with similarly large cephalopelvic dimensions), the possibility that large body size dimorphism may have also affected male sacral morphology (as in gorillas), leads to the possibility that dimorphism in relative sacral breadth in $N$. larvatus may be the result of divergent selective pressures placed on male and female sacral morphology in this species. In other words, obstetric demands may have placed a selective pressure on females of this species for relatively broader sacra, 
while, simultaneously, factors related to increased body size (locomotor demands, etc.) may have selected for relatively narrower sacra in males. Similar arguments for the appearance of sexual dimorphism through divergent selective pressures on males and females have previously been made for other features among primates, including body mass and canine tooth size (Plavcan, 2011). However, further examination of dimorphism in relative sacral breadth among other cercopithecines is needed to more definitively determine the underlying factors driving sexual dimorphism in relative sacral breadth in $N$. larvatus.

Interestingly, Nasalis larvatus is also unusual in that it is the only species in this study to display dimorphism in relative lumbar breadth, with females being significantly larger than males. While the reason for sexual dimorphism in relative lumbar breadth among $N$. larvatus is unclear, it is plausible that either phylogeny or locomotion plays a role in shaping this lumbar morphology, as $N$. larvatus represents the only cercopithecine species in the sample, as well as the only species which employs a palmigrade/plantigrade quadrupedal locomotor pattern. Alternatively, it is also possible that dimorphism in relative lumbar breadth represents a transition to the dimorphic sacral morphology in the vertebral column of this species; however, it should be noted that neither Gorilla gorilla, Hylobates lar, nor Homo sapiens, which also display sexual dimorphism in relative sacral breadth, exhibit sexual dimorphism in relative lumbar breadth. Nonetheless, further research is needed to identify the underlying mechanisms which produce sexually dimorphic lumbar morphology of this species.

Wide sacral alae in females of catarrhine species with large cephalopelvic proportions is most likely related to maintaining obstetrically sufficient pelvic inlet 
dimensions. Although the relationship between relative sacral breadth and transverse breadth of the inlet was not directly evaluated in this study, Tague (2007) has previously demonstrated a positive correlation between breadth of the S1 alae and pelvic inlet breadth in humans. Accordingly, given that the biparietal (transverse) diameter of the neonate head is the most obstetrically constrained dimension in nonhuman primates (Schultz, 1949; Leutenegger, 1974), it appears reasonable to suggest that the relatively wide alae among Hylobates lar and Nasalis larvatus females also serves to expand the transverse diameter of the pelvic inlet in these species with large cephalopelvic proportions. In H. sapiens, H. lar, and N. larvatus, females have relatively (controlling for S1 body breadth) wider pelvic inlets compared to males (and in H. lar, females have absolutely wider pelvic inlets compared to males; see Chapter 2), and dimorphism in the breadth of the sacral alae contributes to dimorphism in inlet breadth in these species. Females also possess relatively wider sacra than males in $G$. gorilla, though this is presumably unrelated to obstetrics, and in this species females also possess relatively wider pelvic inlets than males (see Chapter 2). Though males and females are not dimorphic for relative sacral breadth in P. troglodytes, this species does exhibit dimorphism in transverse inlet diameter controlling for S1 body breadth (see Chapter 2). Therefore, dimorphism in inlet breadth in P. troglodytes is not due to dimorphism in relative sacral breadth, but rather is likely due to dimorphism in pubis length in this species (see Chapter 2).

In addition to providing novel insights into sexual dimorphism in living primates, the results of the present study may have implications for making behavioral inferences about fossil anthropoids, including hominins. While it is generally accepted 
that australopiths were characterized by considerably greater levels of body size dimorphism than exhibited by modern humans (McHenry, 1991, 1992; Richmond and Jugers, 1995; Lockwood et al., 1996), the relative size of australopith neonates, and thus obstetric constraints, have been the subject of debate (Tague and Lovejoy, 1986; DeSilva, 2011). Tague (2007) has previously assessed sexual dimorphism in australopith sacra, finding that two sacra hypothesized to belong to females, A.L. 288-1 (Australopithecus afarensis) and Sts 14 (Australopithecus africanus), possessed relatively longer sacral alae than the putatively male Stw 431 (A. africanus) sacrum (for information on these sex attributions see Leutenegger, 1977; Johanson et al., 1982; Tague and Lovejoy, 1998; Toussaint et al., 2003; but see Häusler and Schmid, 1995). However, Tague (2007) contended that the australopith sacrum is not, in fact, dimorphic, as he regarded this sexually dimorphic result as either a statistical artifact of small sample size or due to the possible subadult status of Stw 431 (Berge and Gommery, 1999), and thus that the sacrum of australopiths "was not associated with obstetric imperatives". However, the results of the current study suggest that, even if australopiths were characterized by sexual dimorphism in relative sacral breadth, this dimorphism may not necessarily be indicative of large cephalopelvic proportions. While potential dimorphism in relative sacra breadth among australopiths could be influenced by selection for relatively broader sacral alae among females related to obstetrics (as in Homo sapiens, Hylobates lar and probably Nasalis larvatus), given that australopiths were likely characterized by a large degree of body size dimorphism (McHenry, 1991, 1992; Richmond and Jugers, 1995; Lockwood et al., 1996), it is also possible that dimorphism in relative sacral breadth could be driven by selection on male australopith 
sacra due to factors related to overall body size (as appears likely for Gorilla gorilla). Consequently, should further fossil discoveries corroborate dimorphism in sacral morphology among australopiths, the present study indicates that there is no reason to assume $a$ priori that such dimorphism is predominantly female-driven, or exclusively related to obstetric demand.

Because this study includes only catarrhine species, future research should address whether platyrrhine species with large cephalopelvic proportions, such as Saimiri sciureus and Ateles geoffroyi (Schultz, 1949; Bowden et al., 1967; Hopf, 1967), similarly exhibit sexual dimorphism in relative sacral breadth. Future studies of sexual dimorphism in relative sacral breadth should also attempt to include larger sample sizes for Pan paniscus and Pongo pygmaeus, as this study included only limited samples of each of these species. Moreover, future studies including a more diverse sample of cercopithecine primates would likely further elucidate the roles of cephalopelvic proportions and body size dimorphism on sacral morphology among catarrhines.

The results of this study indicate that obstetric selection (quantified as cephalopelvic proportions) may be related to dimorphism in relative sacral breadth among species with large cephalopelvic proportions. Together with the results of Chapter 2 (which suggest that cephalopelvic proportions do influence the magnitude of dimorphism in the size and shape of the birth canal), these results indicate that obstetric selection plays an important role in modifying the morphology of the birth canal in females compared to the bony equivalent in males. However, it is unknown whether differences in birth canal morphology between males and females translates into dimorphism in other parts of the pelvis (i.e., parts of the pelvis which do not directly 
participate in the birth canal). Therefore, Chapter 4 of the present study examines whether dimorphism in the size and shape of the birth canal is related to dimorphism in the size and shape of the non-obstetric pelvis in anthropoids. 


\begin{tabular}{|c|c|c|c|c|c|c|c|c|}
\hline \multicolumn{9}{|l|}{ Table 3.1: Sampled taxa } \\
\hline \multirow[t]{2}{*}{ Species } & \multicolumn{2}{|c|}{$\begin{array}{c}\text { n } \\
\text { Lumbar }\end{array}$} & \multicolumn{2}{|c|}{$\begin{array}{c}\text { n } \\
\text { Sacral }\end{array}$} & \multirow{2}{*}{$\begin{array}{c}\text { Body mass } \\
\text { dimorphism } \\
\text { index* } \\
(\mathrm{M} / \mathrm{F})\end{array}$} & \multirow{2}{*}{$\begin{array}{c}\text { Lumbar } \\
\text { body } \\
\text { dimorphism }^{\ddagger} \\
\text { index }^{\ddagger}\end{array}$} & \multirow{2}{*}{$\begin{array}{c}\text { Cephalo- } \\
\text { pelvic } \\
\text { index }^{\dagger}\end{array}$} & \multirow{2}{*}{$\begin{array}{c}\text { Body size } \\
\text { dimorphism } \\
\text { to } \\
\text { cephalopelvic } \\
\text { category }^{+}\end{array}$} \\
\hline & $\mathbf{F}$ & $\mathbf{M}$ & $\mathbf{F}$ & $\mathbf{M}$ & & & & \\
\hline Homo sapiens $^{1}$ & 41 & 30 & 18 & 25 & $1.14^{\S}$ & 1.23 & $1.02^{\ddagger}$ & $\mathrm{A}$ \\
\hline Hylobates lar ${ }^{2}$ & 27 & 38 & 29 & 40 & 1.11 & 1.06 & 0.94 & A \\
\hline Pan paniscus ${ }^{3}$ & 4 & 2 & 6 & 7 & 1.36 & 1.00 & 0.72 & B \\
\hline Pan troglodytes ${ }^{2,4,5}$ & 13 & 10 & 13 & 14 & 1.30 & 1.12 & 0.72 & B \\
\hline Nasalis larvatus ${ }^{2,5}$ & 15 & 12 & 15 & 11 & 2.08 & 1.86 & 0.95 & $\mathrm{C}$ \\
\hline Gorilla gorilla $^{2,4,5}$ & 21 & 20 & 21 & 23 & 2.38 & 1.91 & 0.64 & $\mathrm{D}$ \\
\hline Pongo pygmaeus ${ }^{2,4,5,6}$ & 7 & 7 & 7 & 8 & 2.19 & 1.69 & 0.73 & $\mathrm{D}$ \\
\hline
\end{tabular}

1, University of Iowa-Stanford Collection; 2, Museum of Comparative Zoology; 3, Royal Museum of Central Africa; 4, Cleveland Museum of Natural History; 5, American Museum of Natural History; 6, National Museum of Natural History.

* Values for body size dimorphism based on body mass values from Smith and Jungers (1997).

${ }^{\ddagger}$ Values for lumbar vertebral body dimorphism index are based on the values for average male lumbar vertebral body width divided by the average female lumbar vertebral body width obtained in this study and are calculated as [(male mean lumbar vertebral body breadth/female mean lumbar vertebral body breadth $\left.)^{3}\right]$.

${ }^{\dagger}$ Calculated as (neonate head breadth/maternal pelvic inlet transverse diameter). Values for neonate head and maternal inlet from Schultz (1949).

${ }^{+}$Categories defined as: (A) minimal body size dimorphism and large cephalopelvic proportions; (B) minimal body size dimorphism and small cephalopelvic proportions; (C) large body size dimorphism and large cephalopelvic proportions; (D) large body size dimorphism and small cephalopelvic proportions.

$\S$ Worldwide average body size dimorphism for humans (Smith and Jungers, 1997).

$\$$ The sagittal diameter of the neonate head and transverse breadth of the maternal inlet are used to build this ratio because this is how the human neonate modally engages the inlet.

Sample sizes differ between lumbar and sacral vertebrae because not all specimens included retained both a measurable sacrum and caudal-most lumbar vertebra. 


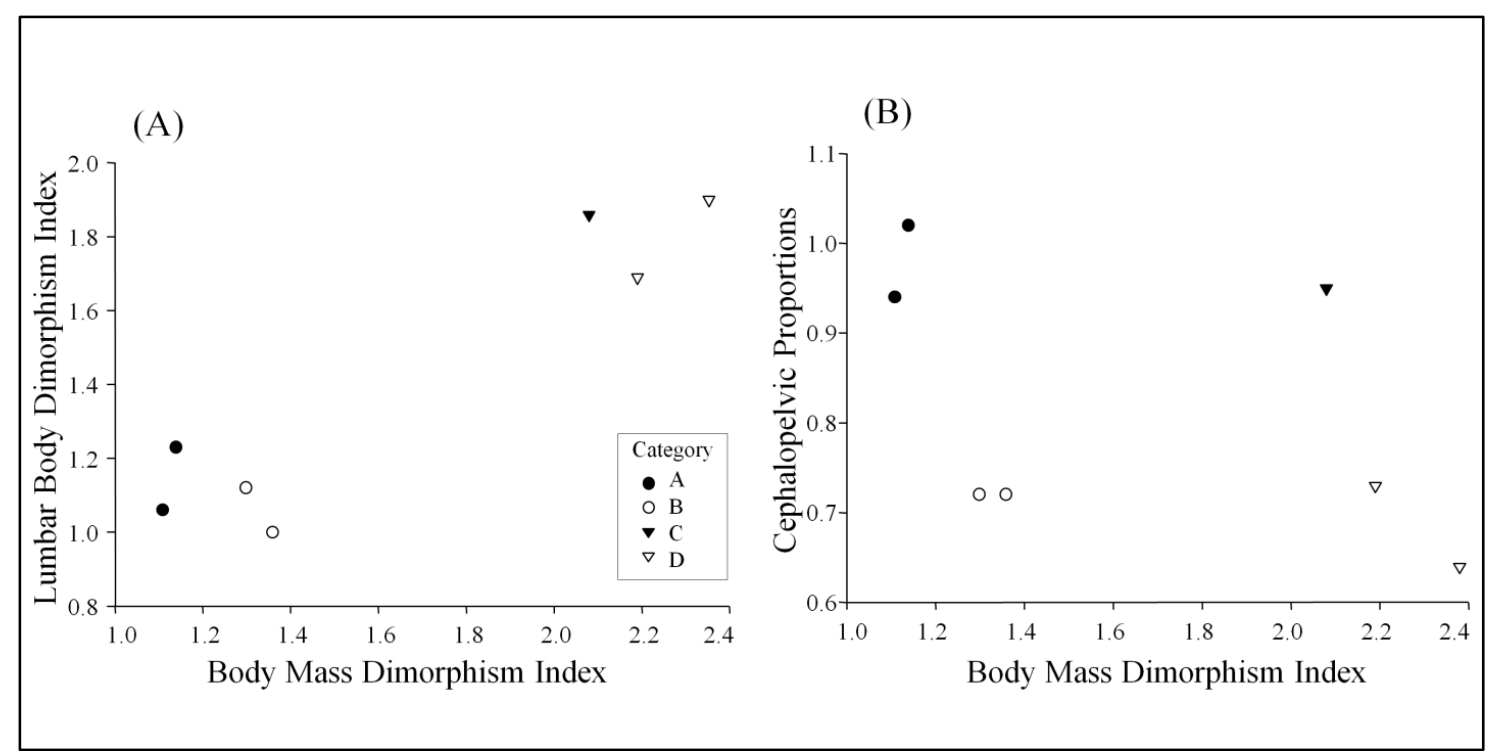

Figure 3.1: Scatter plots of (A) body mass dimorphism index versus lumbar vertebral body dimorphism index and (B) body mass dimorphism index versus cephalopelvic proportions. Categories defined as: A, minimal body size dimorphism and large cephalopelvic proportions (includes Homo sapiens and Hylobates lar); B, minimal body size dimorphism and small cephalopelvic proportions (Pan troglodytes and Pan paniscus); C, large body size dimorphism and large cephalopelvic proportions (Nasalis larvatus); D, large body size dimorphism and small cephalopelvic proportions (Gorilla gorilla and Pongo pygmaeus). 


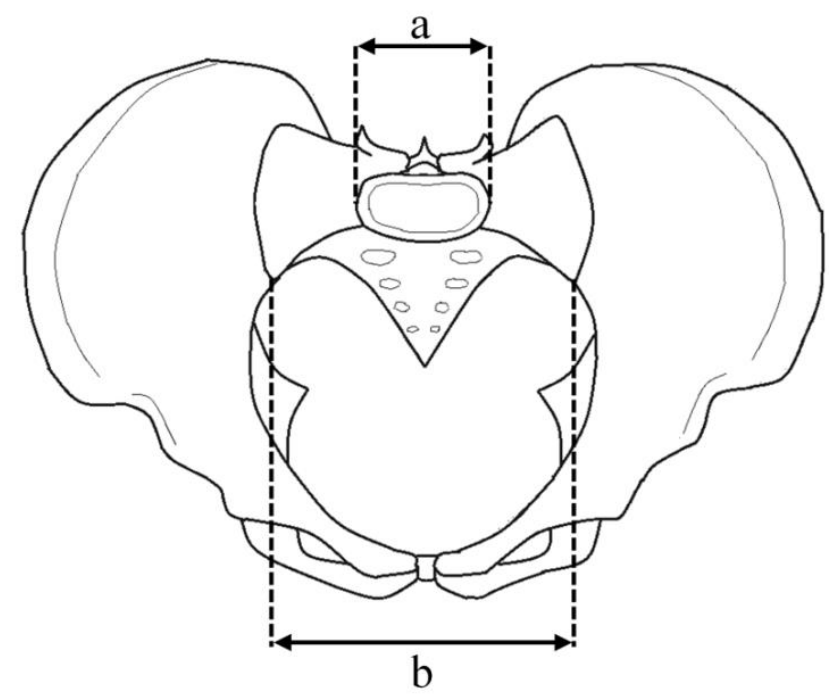

(A)

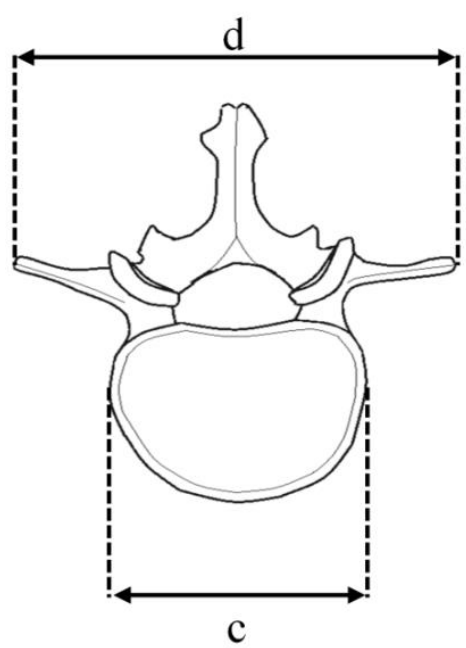

(B)

Figure 3.2: Measurements of the (A) sacrum and (B) lumbar vertebra. Linear measurements are: a) S1 vertebral body width; b) sacral breadth at linea terminalis; c) caudal-most lumbar vertebral body width; and d) caudal-most lumbar bi-transverse process breadth. 
Table 3.2: Isometry test results

In S1 body breadth vs. In sacral breadth at linea terminalis

\begin{tabular}{lcccl}
\hline Group & $\mathbf{N}$ & Slope & $\mathbf{9 5 \%}$ CI & $\mathbf{R}^{\mathbf{2}}$ \\
\hline All species & 265 & 1.111 & $1.072-1.150$ & 0.9154 \\
All females & 133 & 1.154 & $1.098-1.209$ & 0.9226 \\
All males & 132 & 1.072 & $1.025-1.119$ & 0.9366 \\
All hominoids & 239 & 1.100 & $1.059-1.141$ & 0.9145 \\
All hominoid females & 118 & 1.128 & $1.068-1.189$ & 0.9155 \\
All hominoid males & 121 & 1.066 & $1.018-1.113$ & 0.9398 \\
All non-humans & 194 & $0.984^{*}$ & $0.943-1.024$ & 0.9159 \\
All non-human females & 92 & $0.992^{*}$ & $0.934-1.050$ & 0.9222 \\
All non-human males & 102 & $0.985^{*}$ & $0.933-1.037$ & 0.9293 \\
\hline ln lumbar body breadth vs. In lumbar bi-costal breadth & $\mathbf{9 5 \%}$ CI & $\mathbf{R}^{2}$ \\
\hline Group & $\mathbf{N}$ & Slope & $0.897-0.981$ & 0.8855 \\
\hline All species & 220 & 0.939 & $0.837-0.971$ & 0.8577 \\
All females & 105 & 0.904 & $0.907-1.019$ & 0.9025 \\
All males & 115 & $0.963 *$ & $0.928-1.010$ & 0.9110 \\
All hominoids & 193 & $0.969 *$ & $0.892-1.023$ & 0.8958 \\
All hominoid females & 90 & $0.958^{*}$ & $0.920-1.029$ & 0.9195 \\
All hominoid males & 103 & $0.974 *$ & $0.799-0.892$ & 0.8630 \\
All non-humans & 177 & 0.846 & $0.727-0.873$ & 0.8211 \\
All non-human females & 87 & 0.800 & $0.814-0.937$ & 0.8900 \\
All non-human males & 90 & 0.875 & & \\
\hline
\end{tabular}

*Confidence interval includes isometry. 
Table 3.3: Hypotheses and predictions outlined in text

\begin{tabular}{lll}
\hline Hypothesis: & Predictions: & Criteria for Rejection:
\end{tabular}

$\mathbf{H}_{\mathbf{1}}$ : Dimorphism in relative sacral No dimorphism in relative sacral breadth in Any non-human species with breadth not present non-human species

dimorphism in relative sacral breadth

$\mathbf{H}_{2}$ : Dimorphism in relative sacral Dimorphism in relative sacral breadth breadth due to body size dimorphism exclusively in species with moderate to high body size dimorphism

Any minimally dimorphic species having dimorphism in relative sacral breadth

$\mathbf{H}_{3}$ : Dimorphism in relative sacral Dimorphism in relative sacral breadth breadth due to cephalopelvic proportions exclusively in species with large cephalopelvic proportions

$\mathbf{H}_{4}$ : Sexual dimorphism in relative Sexual dimorphism in relative sacral sacral breadth not exclusively related to body size dimorphism breadth related to a combination of both or cephalopelvic proportions body size dimorphism level and cephalopelvic proportions, and/or to phylogeny

Any species with small cephalopelvic proportions having dimorphism in relative sacral breadth

Dimorphism in relative sacral breadth exclusively related either to body size dimorphism or to cephalopelvic proportions 


\begin{tabular}{|c|c|c|c|c|c|}
\hline \multicolumn{6}{|c|}{ S1 vertebral body width } \\
\hline \multirow[b]{2}{*}{ Species } & \multicolumn{2}{|c|}{ Female } & \multicolumn{2}{|c|}{ Male } & \multirow[b]{2}{*}{ p-value } \\
\hline & Mean & S.D. & Mean & S.D. & \\
\hline H. sapiens & 47.59 & 4.86 & 52.84 & 5.34 & $<0.001 *$ \\
\hline H. lar & 19.50 & 1.28 & 19.86 & 1.25 & 0.238 \\
\hline P. paniscus & 31.69 & 2.12 & 31.45 & 2.08 & 0.849 \\
\hline P. troglodytes & 34.50 & 3.58 & 35.79 & 3.87 & 0.377 \\
\hline N. larvatus & 23.19 & 1.17 & 28.91 & 2.52 & $<0.001 *$ \\
\hline G. gorilla & 41.85 & 3.75 & 50.98 & 3.64 & $<0.001 *$ \\
\hline P.pygmaeus & 35.57 & 5.03 & 39.04 & 3.79 & 0.150 \\
\hline \multicolumn{6}{|c|}{ Sacral breadth at linea terminalis } \\
\hline & \multicolumn{2}{|c|}{ Female } & \multicolumn{2}{|c|}{ Male } & \\
\hline & Mean & S.D. & Mean & S.D. & p-value \\
\hline H. sapiens & 108.68 & 6.70 & 107.13 & 6.55 & 0.335 \\
\hline H. lar & 38.40 & 2.75 & 35.36 & 2.06 & $<0.001 *$ \\
\hline P. paniscus & 61.45 & 5.80 & 60.87 & 5.30 & 0.823 \\
\hline P. troglodytes & 64.75 & 5.73 & 63.45 & 6.73 & 0.594 \\
\hline$N$. larvatus & 41.92 & 2.72 & 48.89 & 2.98 & $<0.001 *$ \\
\hline G. gorilla & 78.99 & 6.79 & 86.63 & 7.28 & 0.001* \\
\hline P. pygmaeus & 74.14 & 9.91 & 78.12 & 2.14 & 0.297 \\
\hline \multicolumn{6}{|c|}{ Lumbar vertebral body width } \\
\hline & \multicolumn{2}{|c|}{ Female } & \multicolumn{2}{|c|}{ Male } & \\
\hline & Mean & S.D. & Mean & S.D. & p-value \\
\hline H. sapiens & 50.77 & 4.29 & 54.48 & 4.60 & $0.011 *$ \\
\hline H. lar & 20.43 & 1.07 & 20.87 & 1.09 & 0.115 \\
\hline P. paniscus & 31.04 & 0.54 & 31.05 & 2.06 & 0.939 \\
\hline P. troglodytes & 36.47 & 2.50 & 38.17 & 3.84 & 0.207 \\
\hline$N$. larvatus & 23.30 & 1.22 & 28.72 & 1.84 & $<0.001 *$ \\
\hline G. gorilla & 44.87 & 3.81 & 56.26 & 3.71 & $<0.001 *$ \\
\hline P. pygmaeus & 37.38 & 5.20 & 44.40 & 1.99 & $0.011 *$ \\
\hline \multicolumn{6}{|c|}{ Lumbar bi-transverse process breadth } \\
\hline & \multicolumn{2}{|c|}{ Female } & \multicolumn{2}{|c|}{ Male } & \\
\hline & Mean & S.D. & Mean & S.D. & p-value \\
\hline H. sapiens & 86.40 & 6.53 & 93.84 & 7.64 & $0.002 *$ \\
\hline H. lar & 34.14 & 2.38 & 34.17 & 2.75 & 0.968 \\
\hline P. paniscus & 47.20 & 4.09 & 56.98 & 10.25 & 0.171 \\
\hline P. troglodytes & 56.04 & 5.52 & 55.86 & 7.31 & 0.945 \\
\hline N. larvatus & 46.51 & 3.41 & 54.75 & 4.46 & $<0.001 *$ \\
\hline G. gorilla & 62.68 & 7.32 & 77.26 & 8.74 & $<0.001 *$ \\
\hline P. pygmaeus & 60.98 & 9.82 & 73.22 & 7.06 & $0.017 *$ \\
\hline
\end{tabular}

*Denotes significance at $\alpha=0.05$. 


\begin{tabular}{|c|c|c|c|c|c|c|c|}
\hline \multirow[t]{2}{*}{ Species } & \multirow[t]{2}{*}{ Category* } & \multicolumn{2}{|c|}{$\begin{array}{c}\text { Mean } \\
\text { relative sacral } \\
\text { breadth }\end{array}$} & \multirow[t]{2}{*}{ p-value ${ }^{\dagger}$} & \multicolumn{2}{|c|}{$\begin{array}{c}\text { Mean } \\
\text { relative lumbar } \\
\text { breadth }\end{array}$} & \multirow[t]{2}{*}{ p-value } \\
\hline & & $\mathbf{F}$ & $\mathbf{M}$ & & $\mathbf{F}$ & M & \\
\hline H. sapiens & A & 2.27 & 2.02 & $<0.001^{*}$ & 1.69 & 1.73 & 0.695 \\
\hline H. lar & A & 1.97 & 1.78 & $<0.001 *$ & 1.67 & 1.65 & 0.290 \\
\hline P. paniscus & B & 1.93 & 1.96 & 0.991 & 1.52 & 1.83 & 0.135 \\
\hline P. troglodytes & B & 1.90 & 1.93 & 0.221 & 1.54 & 1.49 & 0.266 \\
\hline N. larvatus & $\mathrm{C}$ & 1.81 & 1.70 & $0.019 *$ & 2.00 & 1.91 & 0.033 \\
\hline G. gorilla & $\mathrm{D}$ & 2.07 & 1.87 & $<0.001 *$ & 1.40 & 1.37 & 0.588 \\
\hline P. pygmaeus & D & 2.03 & 2.02 & 0.442 & 1.63 & 1.66 & 0.831 \\
\hline
\end{tabular}

*Denotes significance at $\alpha=0.05$.

*Categories defined as: (A) minimal body size dimorphism and large cephalopelvic proportions; (B) minimal body size dimorphism and small cephalopelvic proportions; (C) large body size dimorphism and large cephalopelvic proportions; (D) large body size dimorphism and small cephalopelvic proportions.

${ }^{\dagger}$ While raw values for relative sacral breadth are shown in the table, p-values are calculated using natural-logged values for relative sacral breadth and relative lumbar breadth. 


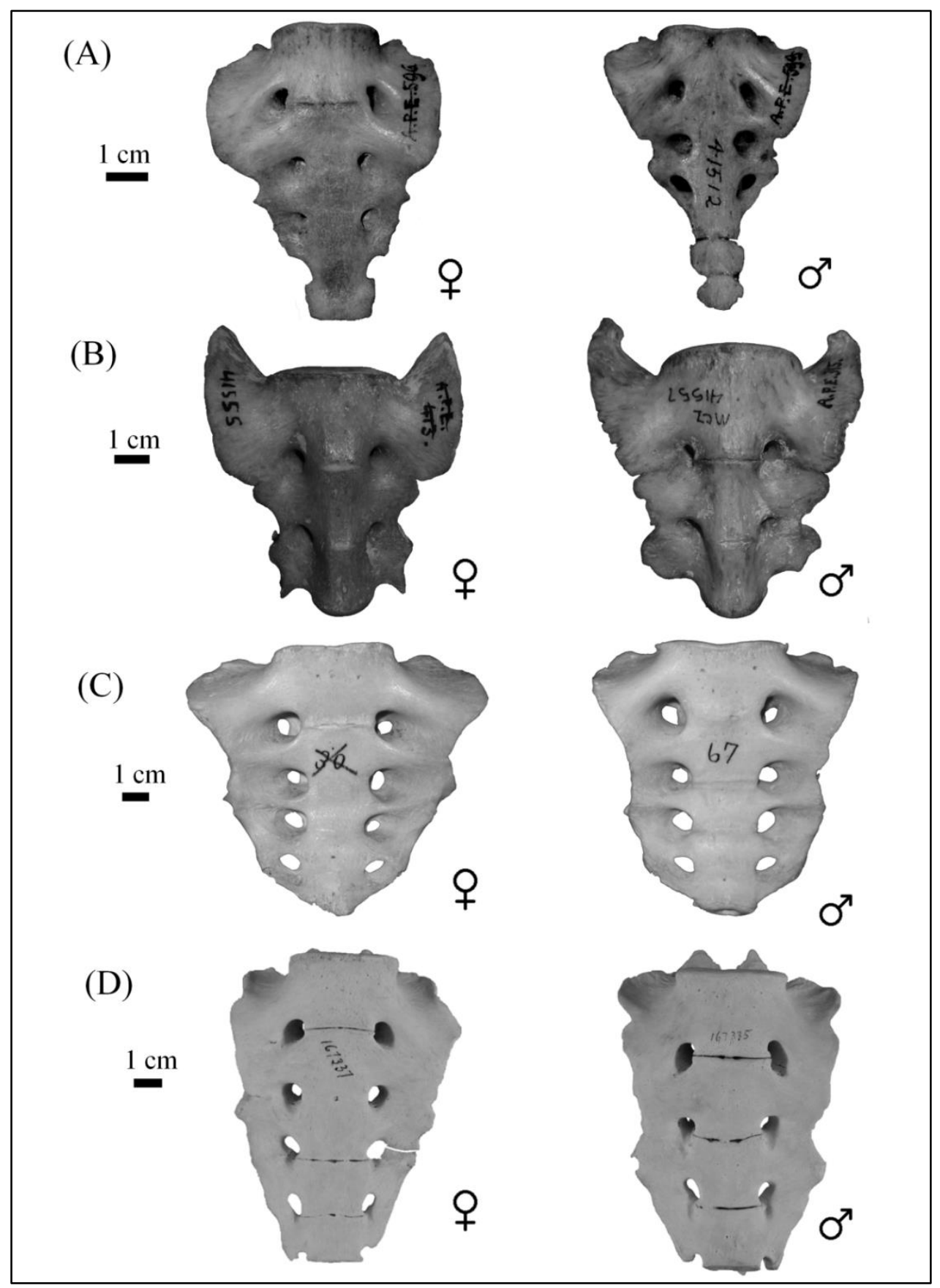

Figure 3.3: Anterior views of male and female sacra of: (a) Hylobates lar; (b) Nasalis larvatus; (c) Homo sapiens; (d) Gorilla gorilla. Note the relative lengthening of the sacral alae among females compared to males. Specimens depicted are: 41512 and 41523 (H. lar; Harvard Museum of Comparative Zoology); 41555 and 41557 ( N. larvatus; Harvard Museum of Comparative Zoology); MU 30 and 67 (H. sapiens; University of Missouri Anatomy Osteology Collection); 167335 and 167337 ( $G$. gorilla; American Museum of Natural History; gorilla photos courtesy of Gabrielle Russo). 


\section{CHAPTER 4: DIMORPHISM IN NON-OBSTETRIC PELVIC MORPHOLOGY IN ANTHROPOIDS}

\section{BACKGROUND:}

Dimorphism in the size and shape of the parts of the pelvis that form the bony birth canal (hereafter referred to as the birth canal) is nearly ubiquitous in anthropoid primates (see Chapter 2; see also Schultz, 1949; Leutenegger, 1974; Steudel, 1981a; Tague, 1991; Ridley, 1995; Tague, 2005). Females have birth canals that are relatively larger (measured as centroid size of the birth canal and circumferences of the inlet, midplane, and outlet) compared to males controlling for body size. However, female birth canals are not just isometrically scaled up versions of those of males - their birth canals are also relatively wider, but not necessarily dorsoventrally expanded, meaning females tend to have rounder birth canals compared to those of males (see Chapter 2) due to a wider sacrum and longer pubis. Species with large cephalopelvic proportions (relatively large neonates) are especially dimorphic (Chapter 2; see also Ridley, 1995).

Dimorphism in birth canal size and shape means that there also must be size and/or shape dimorphism in parts of the pelvis not directly related to birth, termed here the "non-obstetric pelvis". There are three ways that dimorphism in birth canal morphology might manifest itself in other parts of the pelvis. 
First, in most species (all except for Alouatta senciulus; Chapter 2), females have relatively larger birth canals than males. So, it is possible that the non-obstetric parts of female pelves are also larger than those of males, but that the shapes of their non-obstetric pelves remain the same. This would result in both (a) overall non-obstetric pelvic size (centroid size) being larger in females controlling for body size and (b) all or most linear distances of the non-obstetric pelvis being proportionately larger in females compared to males with no shape differences between sexes. This effect should be greatest in species with large cephalopelvic proportions, as they have more dimorphic birth canal size than those with small proportions (see Chapter 2). If this is the case, non-obstetric pelvic size dimorphism would be comparable to obstetric dimorphism.

A second option not mutually exclusive to the first scenario is that pelvic shape might differ between sexes. Females not only have larger birth canals than males, they also have relatively wider inlets, midplanes and/or outlets (all except $A$. seniculus) compared to rostrocaudal (dorsoventral) diameters. This effect is greatest in species with large cephalopelvic proportions. Therefore, the second possibility is that females have non-obstetric pelves that are mediolaterally wider than those of males, but that rostrocaudal dimensions of the non-obstetric pelvis are similar. If so, biacetabular and biiliac breadths should be wider in females (Ruff and Hayes, 1983; Ruff, 1987, 1995; Lovejoy, 2005), but rostrocaudal dimensions of the pelvis (e.g., the distance between the superior ilium and inferior ischium) should be similar controlling for body size. This effect would be especially pronounced in species with large cephalopelvic proportions (see Chapter 2). It is also possible that there is some shape dimorphism in the nonobstetric pelvis that is unrelated to birth canal dimorphism, especially in species with 
high levels of body size dimorphism in which there may be slight differences in locomotor repetoire or ecological niches between sexes (see also Steudel, 1981b; Moffett et al., 2013).

The existence of dimorphism in the birth canals of non-human primates presents an opportunity to test (a) the hypothesis that dimorphism in the size and shape of the non-obstetric pelvis is the result of dimorphism in the birth canal, and (b) the hypothesis that obstetric selection (measured as cephalopelvic proportions) affects dimorphism in non-obstetric pelvic morphology. If dimorphism in the non-obstetric pelvis is a direct result of dimorphism in birth canal size and/or shape, this would increase the information that can be gleaned from fossil pelves about sex, birth canal morphology, and tradeoffs between locomotor and obstetric selection, especially in pelves that are fragmentary or do not preserve a complete birth canal.

\section{HYPOTHESES}

The purpose of the current study is to determine the patterns of dimorphism in the shape and size of the non-obstetric pelvis across anthropoid primates, and to determine whether the magnitude of dimorphism in non-obstetric pelvic size and shape is influenced by cephalopelvic proportions. This study tests the following hypotheses:

Hypothesis 1: Dimorphism in the size of the non-obstetric pelvis reflects dimorphism in birth canal size.

Prediction 1a: In species that have dimorphism in birth canal centroid size controlling for body size (all taxa except Alouatta seniculus and 
Gorilla gorilla), females will also have larger non-obstetric pelvic centroid sizes than males controlling for body size.

Prediction 1b: Species with large cephalopelvic proportions will have a higher magnitude of dimorphism in relative pelvic size compared to species with small proportions.

Hypothesis 2: Dimorphism in the 3D shape of the non-obstetric pelvis reflects dimorphism in the shape of the birth canal.

Prediction 2a: In species that exhibit dimorphism in birth canal shape (all taxa except A. seniculus), there is dimorphism in non-obstetric pelvic shape, such that females have more laterally flaring ischia and inferior ilia relative to the midline, wider biiliac breadth, and wider biacetabular breadths.

Prediction 2b: The magnitude of dimorphism in pelvic shape is greater among taxa with large cephalopelvic proportions than in taxa with small proportions.

\section{MATERIALS AND METHODS}

\section{a. Samples}

Samples chosen for the current study were outlined in Chapter 2. This sample comprises a range of cephalopelvic proportions, phylogenetic affinity, locomotor adaptations, and body size dimorphism among extant anthropoid primates. 
Phylogeny may influence pelvic morphology, but samples for too few taxa are available to achieve sufficient power (Blomberg et al., 2003) using formal phylogenetic analyses. However, including species with large and small cephalopelvic proportions within hominoids [Homo sapiens and Hylobates lar (large proportions); Gorilla gorilla and Pan troglodytes (small proportions)] and platyrrhines [Saimiri sciureus (large proportions) and Alouatta seniculus (small proportions)] provides a set of comparisons with which to informally consider phylogeny. Similarly, locomotor repertoire may be an important factor, but locomotion is completely confounded with phylogeny in anthropoids. Thus, no formal tests considering locomotion are performed.

\section{b. Landmarks}

Methods for articulating paired ossa coxae and sacra were outlined in Chapter 2. Landmarks representing the non-obstetric pelvis (Figure 4.1 and described in Table 4.1) included posterior inferior iliac spine, posterior superior iliac spine, superior iliac crest, anterior superior iliac spine, superior acetabulum, inferior acetabulum, acetabulum center, posterior acetabulum, posterior ischial neck, posterior ischial tuberosity, lateral ischial ramus, lateral ischial tuberosity, inferior pubic ramus, inferior pubic symphysis, superior pubic symphysis. Landmark error was assessed using repeated measures (Kohn et al., 1995) as described in Chapter 2. Error averages for all landmarks is less than $0.002 \%$ of centroid size, and error is equally distributed across all axes, indicating that all landmarks can be collected with high repeatability. 


\section{c. Testing Hypothesis 1}

Hypothesis 1 predicts that dimorphism in non-obstetric pelvic size is due to dimorphism in birth canal size. Overall pelvic size was quantified as non-obstetric pelvic centroid size, which was calculated as the square root of the sum of the squared distances between all non-obstetric landmarks (Figure 4.1; Table 4.2) and the centroid of the configuration (Bookstein, 1991) for each individual.

Testing hypotheses about relative pelvic size between sexes requires consideration of body size and allometric scaling of pelvic size with body size. As in Chapter 2, transverse breadth of the first sacral (S1) vertebral body was chosen as a proxy for body size because it is strongly correlated with body mass across primates (Jungers, 1988; 1991). Scaling of non-obstetric dimensions was evaluated following methods described in Chapter 2. Within monkeys, pelvic centroid size scales isometrically ( $95 \%$ confidence interval $(\mathrm{CI})=0.90-1.04$; Table 4.2$)$, but in apes pelvic centroid size scales with slight negative allometry $(\mathrm{CI}=0.82-0.98$; Table 4.2).

Therefore, it was deemed inappropriate to use ratios of non-obstetric pelvic measurements to $\mathrm{S} 1$ body breadth to compare relative pelvic size between males and females within apes, as any dimorphism in these ratios could simply reflect allometric scaling. Instead, analysis of residuals was used, as described below.

Diagonal (RMA) residuals were calculated for each individual from the maleonly (within apes or within monkeys) regression. For each species, the mean residual of males was compared with that for females for each species using Student's t-tests at $\alpha=$ 0.028 (justification for $\alpha$-level discussed below) to assess the presence of dimorphism. 
In order to control for Type I Error in this study, a False Discovery Rate (FDR) procedure (Benjamini and Hochberg, 1995) as described in Chapter 2 was performed on all raw p-values (i.e., all those obtained in Student's t-tests of absolute and relative pelvic dimensions, residuals, DFA, Student's t-tests of PC scores, and EDMA's FORM tests). The FDR procedure indicated that in order to maintain a false discovery rate of $5 \%, \alpha$-level should be set at 0.028 . Therefore, unadjusted $\mathrm{p}$-values are reported in the text, but only those p-values less than 0.028 were considered significant.

Hypothesis 1 also predicts that species with large cephalopelvic proportions will have a higher magnitude of dimorphism in relative pelvic size compared to species with small proportions. The magnitude of dimorphism within each species was calculated as [(mean female residual)-(mean male residual)]. The larger the resulting number, the higher the magnitude of dimorphism between sexes. A more positive number indicates that females were relatively larger than males (i.e., females have larger residuals), while a more negative number indicates that males were relatively larger.

Hypothesis 1 Prediction 1a was considered rejected if all species which have dimorphism in birth canal centroid size (all except Alouatta seniculus) do not also exhibit dimorphism in non-obstetric pelvic centroid size. Further, prediction b was considered falsified if species with large cephalopelvic proportions did not have a higher degree of dimorphism in non-obstetric pelvic centroid size compared to species with small proportions.

\section{d. Testing Hypothesis 2}


Hypothesis 2 Prediction 2a predicts that the existence of dimorphism in the 3D shape of the non-obstetric pelvis is due to dimorphism in birth canal shape. To quantify shape, principal components analysis (PCA) was employed as described in Chapter 2. Landmarks representing the non-obstetric pelvis (Figure 4.1, Table 4.1) were scaled, translated, and rotated using generalized Procrustes analysis and subjected to tangent space projection. All PCAs were conducted using Morphologika 2.5@ software. Methods for determination of which successive principal components (PCs) significantly contributed to shape variability within each species were described in Chapter 2.

To further quantify the degree of overlap in non-obstetric pelvic shapes within each species, a polygon was placed around the male shape distribution (which theoretically represents the shape of the pelvis when there is no obstetric selection) in the principal components plot. Then, the proportion of females that fell within the male distribution was calculated (see also Chapter 2).

Non-obstetric pelvic shape dimorphism for each species was assessed using Student's t-tests performed on raw principal component scores. PC scores of each sex were compared within species for all PCs that encompass significant shape variation (i.e., $>10 \%$ of intraspecies shape variation) as described in Chapter 2 at $\alpha=0.028$ (see justification for $\alpha$-level above). Visualization of shape differences between males and females within each species was facilitated using wireframes.

Euclidean Distance Matrix Analysis's (EDMA) FORM procedure was used to localize shape differences between males and females as described in Chapter 2 . 
Significance of each interlandmark distance was determined using bootstrapped confidence intervals as described in Chapter 2. The FORM procedure also allowed an estimation of the difference between mean forms (i.e., overall form differences between groups). For differences between mean forms using EDMA's FORM procedure, all pvalues $<0.028$ were considered significant (see justification for $\alpha$-level above). The FORM procedure was performed on both unscaled data as well as data scaled to each individual's geometric mean (in order to obtain shape information controlling for size).

Hypothesis 2 Prediction 2a was considered rejected if taxa which exhibit dimorphism in 3D birth canal shape (all except Alouatta seniculus) did not also exhibit dimorphism in the shape of the non-obstetric pelves such that females have mediolaterally broader pelves (wider biiliac and biacetabular breadths and more laterally flaring ischia) compared to males. Further, Prediction $2 \mathrm{~b}$ was also rejected if species with large cephalopelvic proportions did not also have a greater magnitude of dimorphism in non-obstetric pelvic shape compared to species with small proportions.

\section{RESULTS}

\section{a. Dimorphism in Non-Obstetric Pelvic Size}

Hypothesis 1 (prediction a) posits that species which have dimorphism in birth canal centroid size (all except Alouatta seniculus) also have dimorphism in the size of the non-obstetric pelvis. This prediction is supported, because females in all species in this study except for Alouatta seniculus have larger pelves than males controlling for body size (Table 4.3 ). 
Hypothesis 1 also predicts that the magnitude of dimorphism in non-obstetric pelvic size is greater among species with large cephalopelvic proportions compared to those with small proportions. This prediction is also supported because species with large cephalopelvic proportions do tend to have a higher magnitude of dimorphism in non-obstetric pelvic size than those with small proportions. The magnitudes of dimorphism in species with large cephalopelvic proportions range from 0.25 to 0.02 , (with the mean for all larger proportion taxa being 0.10, and females larger than males in all taxa; Table 4.3) while the range for species with small proportions is $0.05-0.01$ (with the mean for small proportion taxa being 0.04; Table 4.3). However, it should also be noted that there is a great deal of overlap in non-obstetric pelvic size between males and females in most taxa except Nasalis larvatus and Papio anubis (See Appendix A).

\section{b. Dimorphism in Non-Obstetric Pelvic Shape}

Hypothesis 2 (prediction a) predicts that species which have dimorphism in the shape of the birth canal (all except Alouatta seniculus) also have dimorphism in the shape of the non-obstetric pelvis. This prediction is supported. Non-obstetric pelvic shape is significantly dimorphic in all taxa except $A$. seniculus and Papio anubis (Table 4.7). This result for $P$. anubis might be related to small female sample size, however, because only $25 \%$ of females fall into male shape space (Table 4.5 ).

Hypothesis 2 prediction b posits that if non-obstetric pelvic shape dimorphism is found in taxa with large and small cephalopelvic proportions, the magnitude of dimorphism should be higher in species with large proportions. This prediction is supported. The magnitude of dimorphism in 3D pelvic shape (Table 4.5, Figure 4.2) 
tends to be much higher in taxa with large cephalopelvic proportions (in which the percentage of females falling in male shape space ranges from $6 \%$ to $60 \%$ ) compared to taxa with small proportions (in which the percentage of females falling in male shape space ranges from $50 \%$ to $78 \%$ ).

All taxa except A. seniculus do exhibit shape dimorphism (i.e., female pelves are not just isometrically scaled up versions of those of males). Generally, females are larger for dimensions that increase the mediolateral dimensions of the pelvis, whereas males are larger for rostrocaudal dimensions (Figures 4.3-4.11). Among most species, females are relatively larger than males for biacetabular breadth (all species except $M$. mulatta and A. seniculus) and length of the caudal pubic ramus (all taxa). In contrast, males are relatively larger than females for relative ischial length (all taxa except $P$. anubis), and pubic symphysis height (all species).

For the ilium, upper ilium height (distance between dorsal caudal and dorsal rostral iliac spines) is relatively larger in males in all taxa except $P$. anubis and $A$. seniculus, but the lower ilium is relatively longer in females among all taxa except $A$. seniculus (Figures 4.3-4.11). This result is likely due to the fact that the upper ilium is spatially separated from the birth canal (so it is not subject to obstetric selection), but the lower ilium laterally bounds the pelvic inlet and midplane, so any increase in length should increase the circumference of the birth canal.

Biiliac breadth is statistically similar between sexes controlling for S1 body breadth in all taxa except for Nasalis larvatus. This linear distance is also strongly positively allometric with body size in anthropoids (Lewton, 2015), so it is possible that there may be sex differences in biiliac breadth when allometry is taken into account. 
This is the case (Table 4.3). Analysis of residuals of biiliac breadth against S1 body breadth indicate that when allometric scaling is taken into account, females have relatively wider biiliac breadth than males (all taxa except Nasalis larvatus, Pan troglodtytes, and Alouatta seniculus).

In Pan troglodytes and Gorilla gorilla, males have a relatively wider ilium breadth (but not biiliac breadth) than females (Figures 4.9-4.10), but this is not the case in any monkey taxa or in humans (Figures 4.3-4.11). It is possible that in these taxa, males do have wider ilia, but that they are slightly more coronally-oriented so that relative biiliac breadth is statistically similar between sexes. A potential explanation for the observation of dimorphism in relative iliac breadth in G. gorilla and P. troglodytes is that both of these are suspensory taxa with moderate (Pan) to high (Gorilla) levels of body size dimorphism. A relatively broader ilium in males creates more area for the attachment of latissimus dorsi, which attaches to the upper crest of the ilium (Waterman, 1929). Therefore, a broader ilium in males could be advantageous for suspensory locomotion at such large body sizes. In contrast, in most monkeys the iliac blades are narrow (Waterman, 1929) and laterally facing (Ward, 1993), which might suggest that iliac breadth (or just body breadth) is more constrained in monkeys than in large bodied apes.

\section{DISCUSSION AND CONCLUSIONS}

Some level of dimorphism in non-obstetric pelvic size and shape exists across most anthropoids, just as is seen in birth canal (see Chapter 2). This is also seen across 
many mammalian taxa, including mice (Gardner, 1936; Schutz et al., 2009a), bats (Crelin and Newton, 1969), foxes (Schutz et al., 2009b), opossums (Tague, 2003), and lorises (Leutenegger, 1973). Thus, it is clear that dimorphism in pelvic size and shape is not relegated to just the birth canal, and dimorphism is not just found in species that give birth to relatively large neonates. Moreover, this may be an ancestral character for primates, or indeed for mammals.

The presence of pelvic dimorphism even outside the birth canal cannot be taken as evidence that a species has rigorous obstetric selection or large cephalopelvic proportions. Yet, it is clear that there is an effect of relative neonate size on the magnitude of dimorphism in pelvic size across anthropoids, even outside the birth canal. Taxa with large cephalopelvic proportions are more dimorphic in non-obstetric pelvic size and shape than species with small proportions, as they are in the parts of the pelvis contributing to the birth canal (see Chapter 2). The magnitude non-obstetric size and shape dimorphism generally corresponds to the magnitude of dimorphism birth canal size and shape. Those taxa that have a high degree of overlap in birth canal size and shape also have a high degree of overlap in non-obstetric pelvic size and shape (see also Appendix A).

Though females do have relatively larger pelves that males, in most species (all those studied here except Alouatta seniculus), there is a great degree of overlap in nonobstetric pelvic size across most taxa (except for Nasalis larvatus and Papio anubis; see Appendix A). Thus, differences in non-obstetric pelvic size may not be observable at small sample sizes in many taxa. This is especially important to consider when 
examining dimorphism in pelvic size in fossil specimens, which will be represented by smaller sample sizes than the species included here.

Though females do have statistically larger non-obstetric pelvic size than males in most taxa, female pelves are not just isometrically larger versions of male pelves. Females tend to have wider pelves, but not rostrocaudally taller pelves than those of males. Biacetabular distance, which is immediately adjacent to the midplane of the birth canal, is dimorphic among most taxa included here (except A. seniculus and Macaca mulatta). Notably, relative midplane breadth is also not dimorphic in either A. seniculus or M. mulatta. Therefore, these results indicate that dimorphism in biacetabular breadth is a result of dimorphism in the breadth of the pelvic midplane. It has been hypothesized that increased biacetabular distance could be energetically costly in bipeds because it will increase the abductor forces required to prevent hip drop during bipedal locomotion when there is not a concurrent increase in femoral neck length (Ruff, 1995). It is reasonable to assume that increased biacetabular breadth would increase the distance between the origin (gluteal plane of ilium; Waterman, 1929) and insertions (greater trochanter of femur) of these muscles. This distance would be further increased by a relatively larger lower iliac height in females (see further discussion below). It is possible that increasing this distance could be biomechanically disadvantageous in nonhuman primates - the lesser gluteal muscles aid in maintaining squatting or upright sitting postures in most primates (Waterman, 1929). Increasing the distance between the center of mass and the attachment of the lesser gluteals will increase their required effort to maintain these postures. Therefore, a wider biacetabular breadth may be 
energetically costly for females among non-human primates. Further research is needed to examine this hypothesis.

Biiliac breadth is strongly positively allometric in primates (Lewton, 2015), so it is unsurprising that females (smaller body size) do not have relatively wider biiliac breadth than males (larger body size) controlling for body size. However, females do have relatively wider biiliac breadth than males when controlling for allometric scaling (Table 4.3). However, this result does indicate that the ischiopubis is disproportionately wider in females than males compared to the upper ilium, which remains statistically similar in breadth between sexes controlling for body size. It is possible that this result also reflects the fact that the upper ilium is spatially separated from the birth canal in primates, so a drastic increase in biiliac breadth in females would not provide a direct obstetric advantage. In contrast, increasing the breadth of the ischiopubis directly affects the size and shape of the birth canal.

Lower iliac height is relatively larger in females than males among all species included here except Alouatta seniculus and Papio anubis. This longer lower iliac height in females compared to males is in accordance with the fact that females also have larger circumferences of the inlet, midplane, and outlet among most species increasing lower iliac height necessarily increases the distance between the sacroiliac joints, acetabulae, and ischia. Further, increasing inferior iliac height also lengthens the distance between the iliac fossae (origins for the gluteus medius and minimus) and the hip joint. A decreased inferior iliac height in bipeds is thought to help combat hip drop during single-leg stance (Lovejoy, 2005; Lovejoy and McCollum, 2010), so increasing this distance could impact this function in human females. In all primates (not just 
humans) lower iliac height is also a rough measurement of the power arm of the gluteal muscles for movement about the hip joint (Waterman, 1929). Additionally, it has been argued that a short iliac breadth is selectively advantageous, especially in primates that have high forces being transmitted through this region of the pelvis (e.g., in leaping primates and in bipeds; Morbeck, 1976; Fleagle, 1978), because a short lower iliac height could decrease the torque produced when the weight of the upper trunk is opposed by upward forces from the lower limb contacting substrate at the acetabulum (Steudel, 1981b). Therefore, dimorphism in lower iliac height among humans and nonhuman primates may be related to the fact that a short lower iliac height (in males) is advantageous from a locomotor standpoint, but a long lower iliac height (in females) is advantageous for birth.

The fact that dimorphism in lower iliac height is (a) concurrent with dimorphism in birth canal size and (b) found in nearly all primates included here (except Alouatta seniculus) holds promise for interpretation of pelvic dimorphism and sex in fossils, even when the birth canal may not be preserved (which is typically the case). Though only a few fossil hominin pelves preserve a complete or nearly complete birth canal - A.L. 288-1 (Au. afarensis; Johansen et al., 1982), BSN49 (probably Homo erectus; Simpson et al., 2008), Kebara 2 (Neandertal; Rak and Arensburg, 1987) - the lower ilium is preserved in many specimens, including Sts 14 (Au. africanus), MH2 (Au. sediba), KNM-ER 3228 and 5881 (early Homo; Rose, 1984; Ward et al., 2015); KNM-WT 15000 (Homo erectus; Brown et al., 1985) and Tabun 1 and Qafzeh 9 (both Neandertal). Unlike the upper ilium and pubis, the region between the auricular surface of the ilium and the acetabulum is frequently preserved in fossil hominins because this area is 
typically quite robust. Because dimorphism in lower ilium height is associated with dimorphism in birth canal size, lower ilium height may be instrumental in increasing the amount of information known about birth canal size in early hominins.

Because dimorphism in the non-obstetric pelvis - including the lower ilium and ischiopubis - appears to be correlated with dimorphism in the birth canal, this implies that the birth canal and non-obstetric pelvis may not be completely independent, though the rostral ilium could be less integrated with other aspects of the pelvis (since this area does not increase in breadth in females to the same extent the ischiopubis does). This decreased integration of some parts of the non-obstetric pelvis with the birth canal could be reflected in how modular the primate pelvis is. This hypothesis is tested in Chapter 5 . Further, both the results of this chapter as well as Chapter 2 indicate that pelvic dimorphism is most pronounced in taxa with large cephalopelvic proportions, and this indicates that obstetric selection might maintain these high pelvic dimorphism levels through selection on female pelvic form. This should result in tighter pelvic integration in females than males, especially in species with large cephalopelvic proportions - a hypothesis that is also tested in Chapter 5. 


\section{Table 4.1: False Pelvis Landmarks}

\section{Bilateral Landmarks}

(1) Posterior inferior iliac spine

(2) Posterior superior iliac spine

(4) Anterior superior iliac spine

(5) Superior acetabulum

(6) Inferior acetabulum

(7) Acetabulum center

(8) Posterior acetabulum

(9) Posterior ischial neck

(10) Posterior ischial tuberosity

(11) Lateral ischial ramus

(12) Lateral ischial tuberosity

(13) Inferior pubic ramus
(3) Superior iliac crest

\section{Description}

Posterior-most point of the posterior inferior iliac spine

Posterior-most point of the posterior superior iliac spine

Superior-most point on the iliac crest

Lateral-most point on the anterior superior iliac spine

Point on superior rim of acetabulum in the same plane as anterior inferior iliac spine

Point on inferior rim of acetabulum in the same plane as ischial ramus

Point in the acetabulum at the center of the lunate surface

Rim of the acetabulum in the same plane as landmarks 7 and 9

Point on the posterior ischium in the same plane as landmarks 8 and 9

Posterior-most point on the ischial tuberosity

Deepest point along the ischial ramus inferior to the acetabulum

Lateral-most projection of the ischial tuberosity

Point between ischial ramus and inferior pubic ramus in same plane as lateral obturator foramen

\section{Midline Landmarks Description}

(14) Inferior pubic symphysis* Inferior-most point on symphysis at the midline

(15) Superior pubic symphysis* Superior-most point on symphysis at the midline

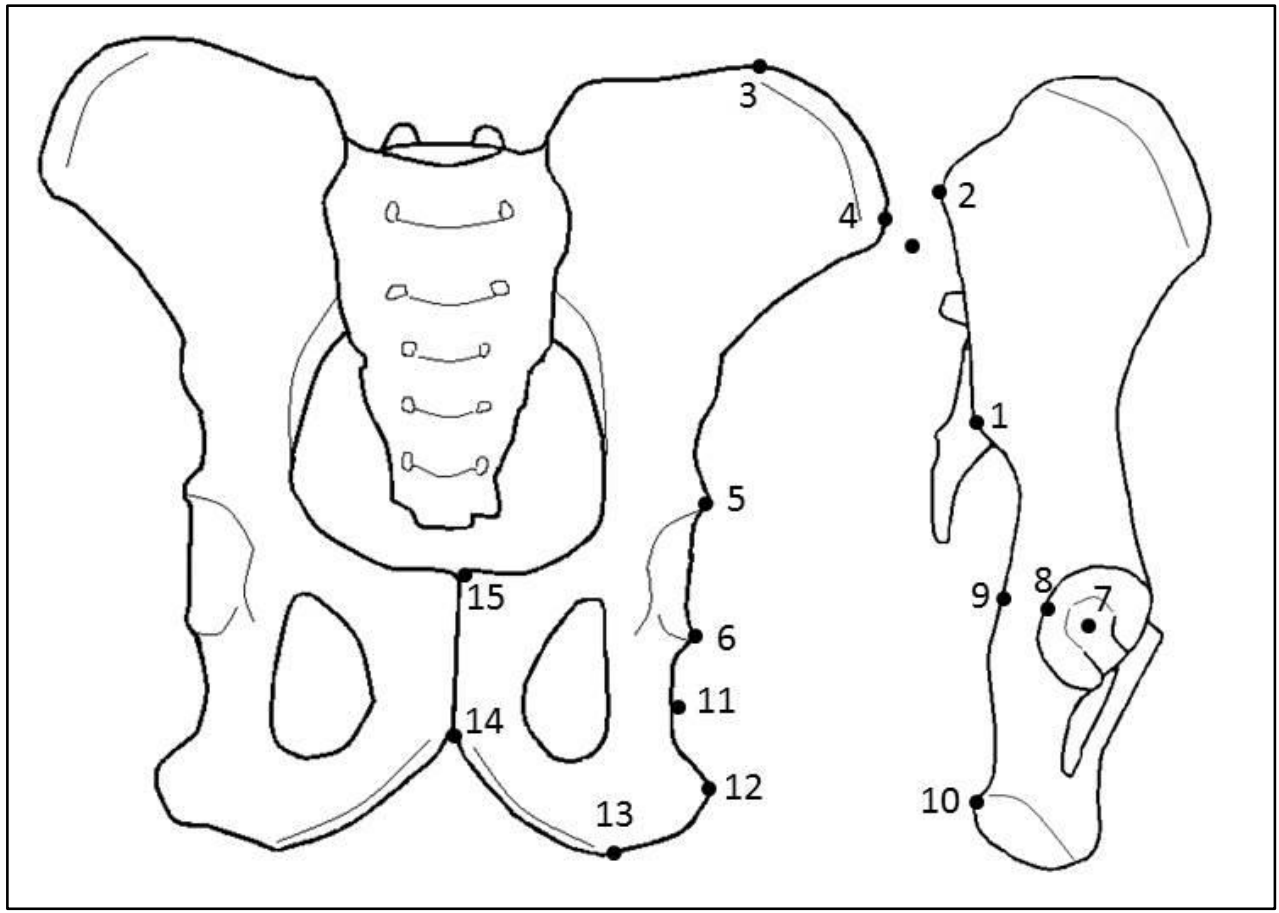

Figure 4.1: Landmarks of the false pelvis: (1) posterior inferior iliac spine; (2) posterior superior iliac spine; (3) superior iliac crest; (4) anterior superior iliac spine; (5) superior acetabulum; (6) inferior acetabulum; (7) acetabulum center; (8) posterior acetabulum; (9) posterior ischial neck; (10) posterior ischial tuberosity; (11) lateral ischial ramus; (12) lateral ischial tuberosity; (13) inferior pubic ramus; (14) inferior pubic symphysis; (15) superior pubic symphysis. 
Table 4.2: Reduced major axis regressions of natural logged non-obstetric pelvis dimensions against logged $S 1$ body breadth *

\begin{tabular}{|c|c|c|c|c|}
\hline & \multicolumn{4}{|c|}{ x S1 Body Breadth } \\
\hline & \multirow{3}{*}{$\mathbf{r}^{2}$} & Slope & Slope & Slope \\
\hline & & Anthropoids & Apes & Monkeys \\
\hline & & (C.I) & (C.I) & (C.I) \\
\hline \multirow{2}{*}{ Pelvic Centroid Size } & \multirow{2}{*}{0.94} & $1.04 *$ & 0.90 & $0.97 *$ \\
\hline & & $(1.00-1.07)$ & $(0.82-0.98)$ & $(0.90-1.04)$ \\
\hline
\end{tabular}

*Denotes isometry.

\begin{tabular}{|c|c|c|c|c|}
\hline \multicolumn{5}{|c|}{ Table 4.3: Dimorphism in non-obstetric pelvic size; Student's t-Tests on residuals } \\
\hline \multirow[b]{2}{*}{ Large Cephalopelvic Proportions } & \multicolumn{4}{|c|}{ Pelvic Centroid Size } \\
\hline & $\begin{array}{c}\text { F } \\
\text { Mean } \\
\text { Residual } \\
\end{array}$ & $\begin{array}{c}\text { M } \\
\text { Mean } \\
\text { Residual } \\
\end{array}$ & $\begin{array}{c}\text { p-value } \\
\text { (sex with more } \\
\text { positive mean } \\
\text { residual) } \\
\end{array}$ & $\begin{array}{c}\text { Magnitude of } \\
\text { Dimorphism } \\
{[(\text { mean female residual })-} \\
\text { (mean male residual })]\end{array}$ \\
\hline Homo sapiens & -0.13 & -0.21 & $<0.001 *(\mathrm{f})$ & 0.08 \\
\hline Hylobates lar & 0.07 & 0.03 & $0.010 *(f)$ & 0.04 \\
\hline Macaca mulatta & 0.14 & 0.12 & $0.008 *(f)$ & 0.02 \\
\hline Nasalis larvatus & 0.18 & -0.07 & $<0.001 *(\mathrm{f})$ & 0.25 \\
\hline Papio anubis & 0.23 & 0.06 & $0.002 *(f)$ & 0.17 \\
\hline Saimiri sciureus & 0.04 & -0.02 & $<0.001 *(\mathrm{f})$ & 0.06 \\
\hline \multicolumn{5}{|l|}{ Small Cephalopelvic Proportions } \\
\hline Gorilla gorilla & 0.15 & 0.10 & $0.024 *(\mathrm{f})$ & 0.05 \\
\hline Pan troglodytes & 0.18 & 0.13 & $0.005 *$ (f) & 0.05 \\
\hline \multirow[t]{3}{*}{ Alouatta seniculus } & -0.45 & -0.44 & 0.453 & 0.01 \\
\hline & \multicolumn{4}{|c|}{ Biiliac Breadth } \\
\hline & $\begin{array}{c}\text { F } \\
\text { Mean } \\
\text { Residual }\end{array}$ & $\begin{array}{c}\text { M } \\
\text { Mean } \\
\text { Residual }\end{array}$ & $\begin{array}{c}\text { p-value } \\
\text { (sex with more } \\
\text { positive mean } \\
\text { residual) }\end{array}$ & $\begin{array}{c}\text { Magnitude of } \\
\text { Dimorphism } \\
\text { [(mean female residual })- \\
\text { (mean male residual })]\end{array}$ \\
\hline Homo sapiens & -0.23 & -0.30 & $0.005 *(\mathrm{f})$ & 0.07 \\
\hline Hylobates lar & 0.12 & 0.05 & $0.008 *(\mathrm{f})$ & 0.07 \\
\hline Macaca mulatta & 0.19 & 0.11 & $<0.001 *$ (f) & 0.08 \\
\hline Nasalis larvatus & 0.26 & 0.17 & 0.034 & 0.09 \\
\hline Papio anubis & 0.26 & 0.14 & $0.006^{*}(\mathrm{f})$ & 0.12 \\
\hline Saimiri sciureus & 0.03 & -0.01 & $0.025 *(f)$ & 0.04 \\
\hline \multicolumn{5}{|l|}{ Small Cephalopelvic Proportions } \\
\hline Gorilla gorilla & 0.29 & 0.17 & $0.003 *(f)$ & 0.12 \\
\hline Pan troglodytes & 0.26 & 0.23 & 0.081 & 0.03 \\
\hline Alouatta seniculus & -0.62 & -0.64 & 0.435 & -0.02 \\
\hline
\end{tabular}

* Denotes significance at $\alpha=0.028$. 


\begin{tabular}{lcl}
\hline Table 4.4: Magnitude of Dimorphism Ranks for the Non-Obstetric Pelvis \\
\hline Species & $\begin{array}{l}\text { Magnitude of Dimorphism in } \\
\text { Residual Pelvic Centroid Size* }\end{array}$ & $\begin{array}{l}\text { Cephalopelvic } \\
\text { Proportions }\end{array}$ \\
\hline Nasalis larvatus & 0.25 & large \\
Papio anubis & 0.17 & large \\
Homo sapiens & 0.08 & large \\
Saimiri sciureus & 0.06 & large \\
Gorilla gorilla & 0.05 & small \\
Pan troglodytes & 0.05 & small \\
Hylobates lar & 0.04 & large \\
Macaca mulatta & 0.02 & large \\
Alouatta seniculus & 0.01 & small \\
\hline
\end{tabular}

*Calculated as $\{($ female mean residual $)$ - (male mean residual $)\}$. 


\begin{tabular}{lc}
\hline Table 4.5: Shape overlap between sexes* \\
\hline Large Proportions & Percent of Females in Male Shape Space \\
\hline Homo sapiens & $23 \%$ \\
Hylobates lar & $60 \%$ \\
Macaca mulatta & $9 \%$ \\
Nasalis larvatus & $6 \%$ \\
Papio anubis & $25 \%$ \\
Saimiri sciureus & $12 \%$ \\
\hline Small Proportions & Percent of Females in Male Shape Space \\
\hline Gorilla gorilla & $50 \%$ \\
Pan troglodytes & $78 \%$ \\
Alouatta seniculus & $67 \%$ \\
\hline
\end{tabular}

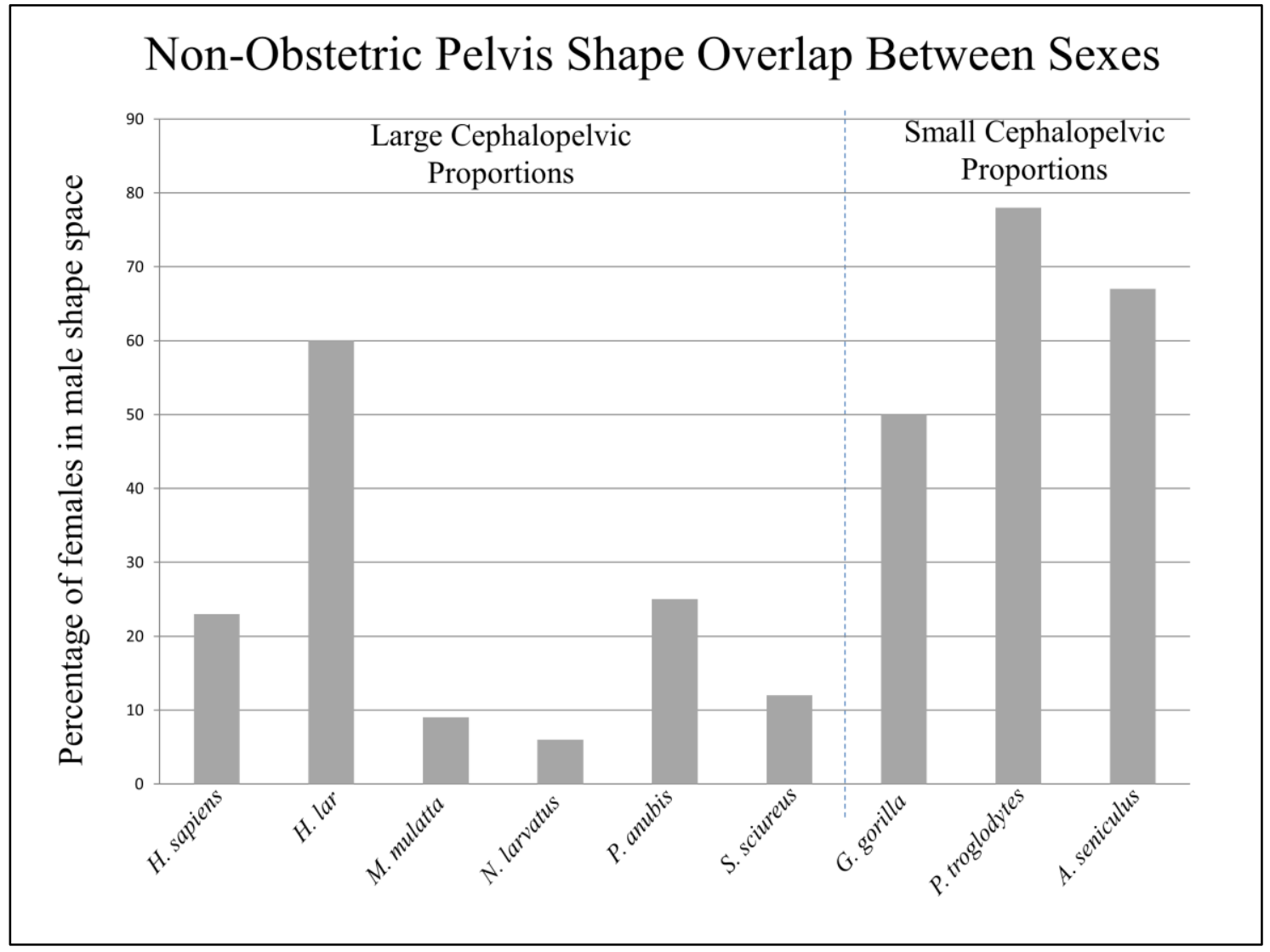

Figure 4.2: Overlap between sexes in 3-D non-obstetric pelvis shape. 


\begin{tabular}{lcccccc}
\hline Table 4.6: Student's t-tests for dimorphism in birth canal shape* & Pariation \\
\hline Large Proportions & $\begin{array}{c}\text { p-value for } \\
\text { PC1 }\end{array}$ & $\begin{array}{c}\text { Variation } \\
\text { explained }\end{array}$ & $\begin{array}{c}\text { p-value for } \\
\text { PC2 }\end{array}$ & $\begin{array}{c}\text { Variation } \\
\text { explained }\end{array}$ & $\begin{array}{c}\text { p-value for } \\
\text { PC3 }\end{array}$ & $\begin{array}{c}\text { Variation } \\
\text { explained }\end{array}$ \\
\hline Homo sapiens & $2.20 \mathrm{E}-16^{*}$ & $26.7 \%$ & $2.72 \mathrm{E}-08^{*}$ & $15.0 \%$ & $6.01 \mathrm{E}-03^{*}$ & $9.1 \%$ \\
Hylobates lar & $1.85 \mathrm{E}-07^{*}$ & $23.0 \%$ & $2.53 \mathrm{E}-02^{*}$ & $9.9 \%$ & $2.19 \mathrm{E}-03^{*}$ & $8.7 \%$ \\
Macaca mulatta & $2.20 \mathrm{E}-16^{*}$ & $24.1 \%$ & $4.19 \mathrm{E}-03^{*}$ & $13.6 \%$ & $9.61 \mathrm{E}-04^{*}$ & $8.9 \%$ \\
Nasalis larvatus & $1.27 \mathrm{E}-09^{*}$ & $27.6 \%$ & $3.98 \mathrm{E}-01$ & $15.2 \%$ & $1.91 \mathrm{E}-01$ & $11.9 \%$ \\
Papio anubis & $3.53 \mathrm{E}-02$ & $20.5 \%$ & $1.61 \mathrm{E}-01$ & $16.9 \%$ & $4.29 \mathrm{E}-01$ & $11.8 \%$ \\
Saimiri sciureus & $1.52 \mathrm{E}-10^{*}$ & $21.7 \%$ & $2.20 \mathrm{E}-16^{*}$ & $12.6 \%$ & $2.88 \mathrm{E}-01$ & $8.3 \%$ \\
\hline & p-value for & Variation & p-value for & Variation & p-value for & Variation \\
Small Proportions & PC1 & explained & PC2 & explained & PC3 & explained \\
\hline Alouatta seniculus & $4.43 \mathrm{E}-02$ & $23.2 \%$ & $9.24 \mathrm{E}-01$ & $14.9 \%$ & $5.07 \mathrm{E}-01$ & $10.2 \%$ \\
Gorilla gorilla & $6.06 \mathrm{E}-01$ & $18.2 \%$ & $8.52 \mathrm{E}-08^{*}$ & $11.8 \%$ & $2.17 \mathrm{E}-06^{*}$ & $10.3 \%$ \\
Pan troglodytes & $2.45 \mathrm{E}-03$ & $16.7 \%$ & $9.30 \mathrm{E}-04^{*}$ & $13.2 \%$ & $2.83 \mathrm{E}-02$ & $9.2 \%$ \\
\hline
\end{tabular}

$*$ Denotes significance at $\alpha=0.028$.

Table 4.7: Euclidean Distance Matrix Analyses of male and female differences in overall non-obstetric pelvis shape within species

\begin{tabular}{lcc}
\hline Large Cephalopelvic Proportions & $\begin{array}{c}\text { Non-Obstetric Pelvis } \\
\text { (unscaled) }\end{array}$ & $\begin{array}{c}\text { Non-Obstetric Pelvis } \\
\text { (scaled to geometric mean) }\end{array}$ \\
\cline { 2 - 3 } p-value & $<$-value \\
\hline Homo sapiens & $<0.001^{*}$ & $<0.001^{*}$ \\
Hylobates lar & $0.007^{*}$ & $0.003^{*}$ \\
Macaca mulatta & $<0.001^{*}$ & $<0.001^{*}$ \\
Nasalis larvatus & $<0.001^{*}$ & $<0.001^{*}$ \\
Papio anubis & 0.029 & 0.032 \\
Saimiri sciureus & $<0.001^{*}$ & $<0.001^{*}$ \\
\hline Small Cephalopelvic Proportions & p-value & p-value \\
\hline Alouatta seniculus & 0.566 & 0.568 \\
Gorilla gorilla & $<0.001^{*}$ & $<0.001^{*}$ \\
Pan troglodytes & $0.017^{*}$ & $0.018^{*}$ \\
\hline
\end{tabular}

*Denotes significance at $\alpha=0.028$. 


\section{Homo sapiens: PCA of non-obstetric landmark data}

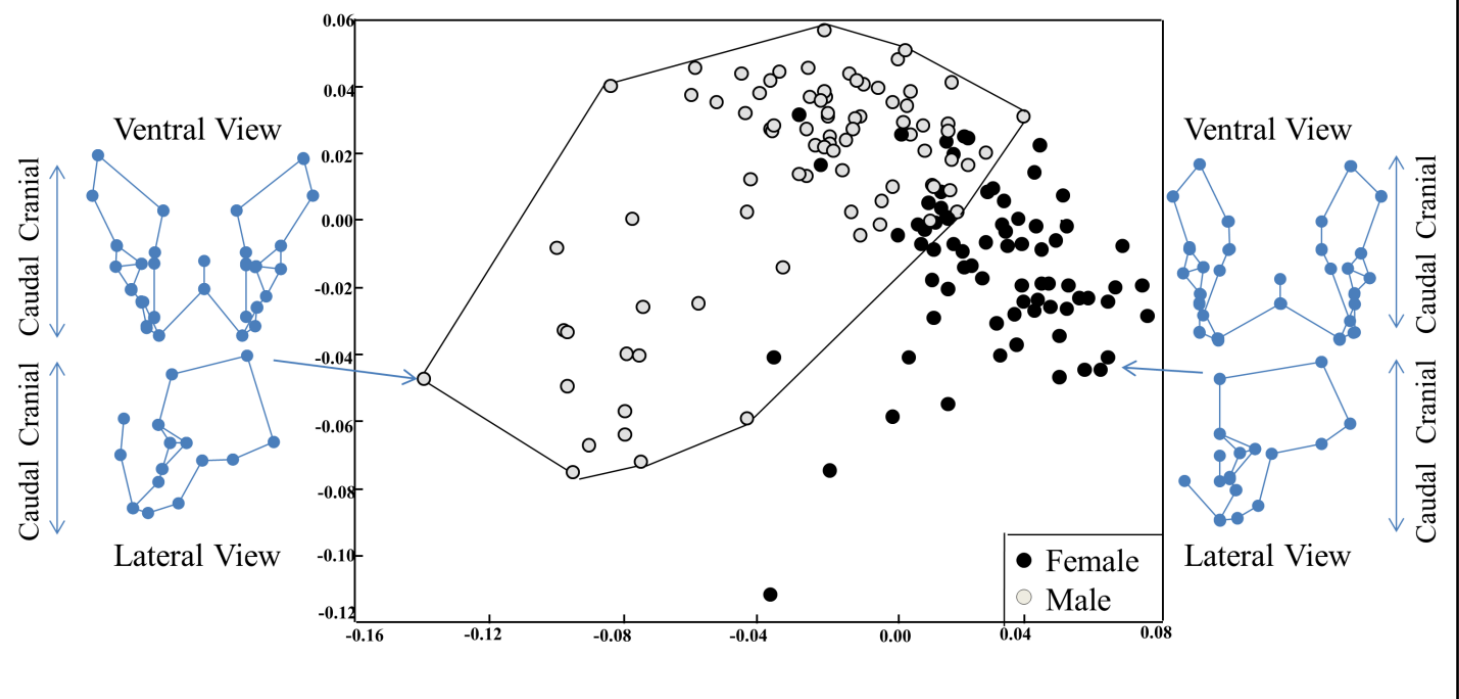

Figure 4.3a: Shape analysis for the false pelvis of Homo sapiens. (a) Principal components analysis of false pelvis landmark data. Note the separate clustering of males and females along the $\mathrm{X}$-axis (PC1).

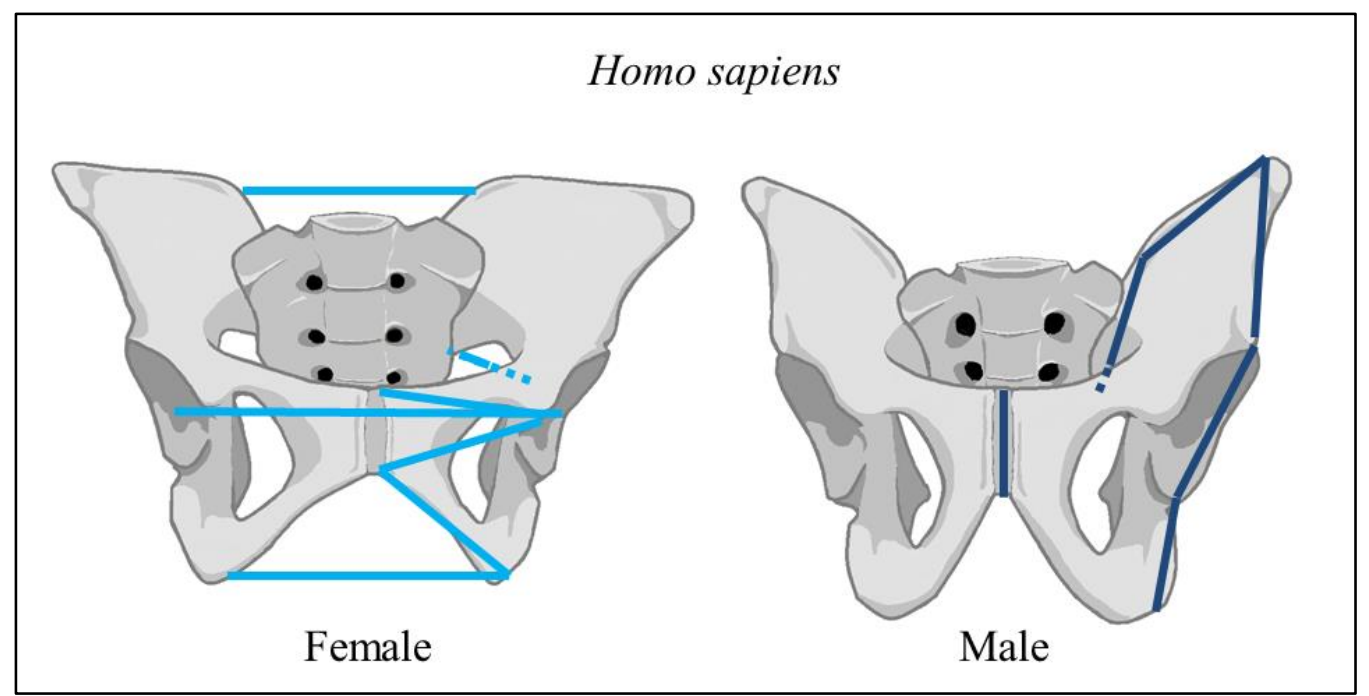

Figure 4.3b: FORM procedure results to obtain dimorphic linear distances in the pelvis of Homo sapiens when pelves are scaled to each individual's geometric mean (body size proxy). Light blue represents linear distances which are greater in females than males. Dark blue represents linear distances which are relatively greater in males compared to females. Only non-overlapping distances are included. 


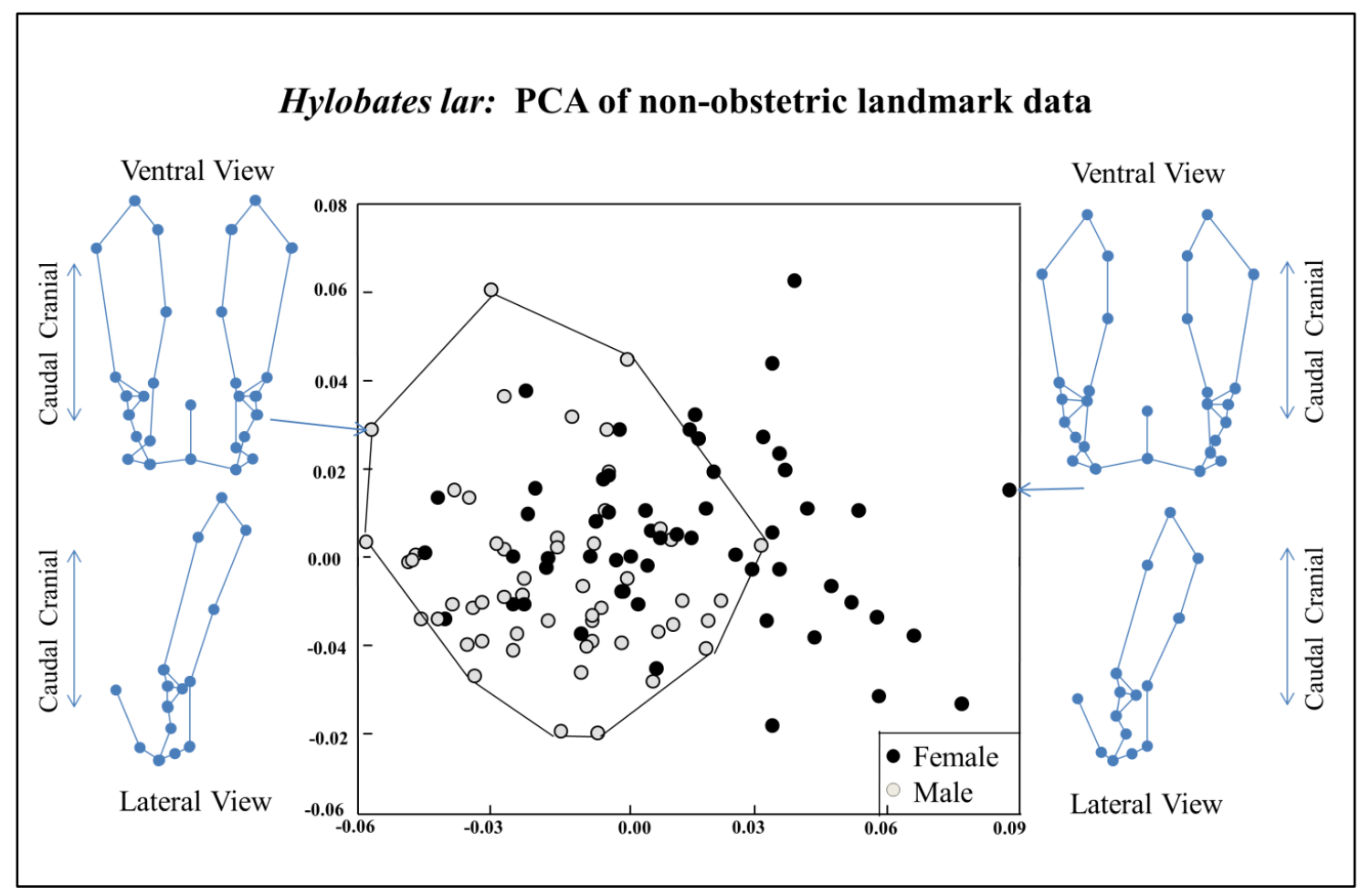

Figure 4.4a: Shape analysis for the false pelvis of Hylobates lar. (a) Principal components analysis of false pelvis landmark data.

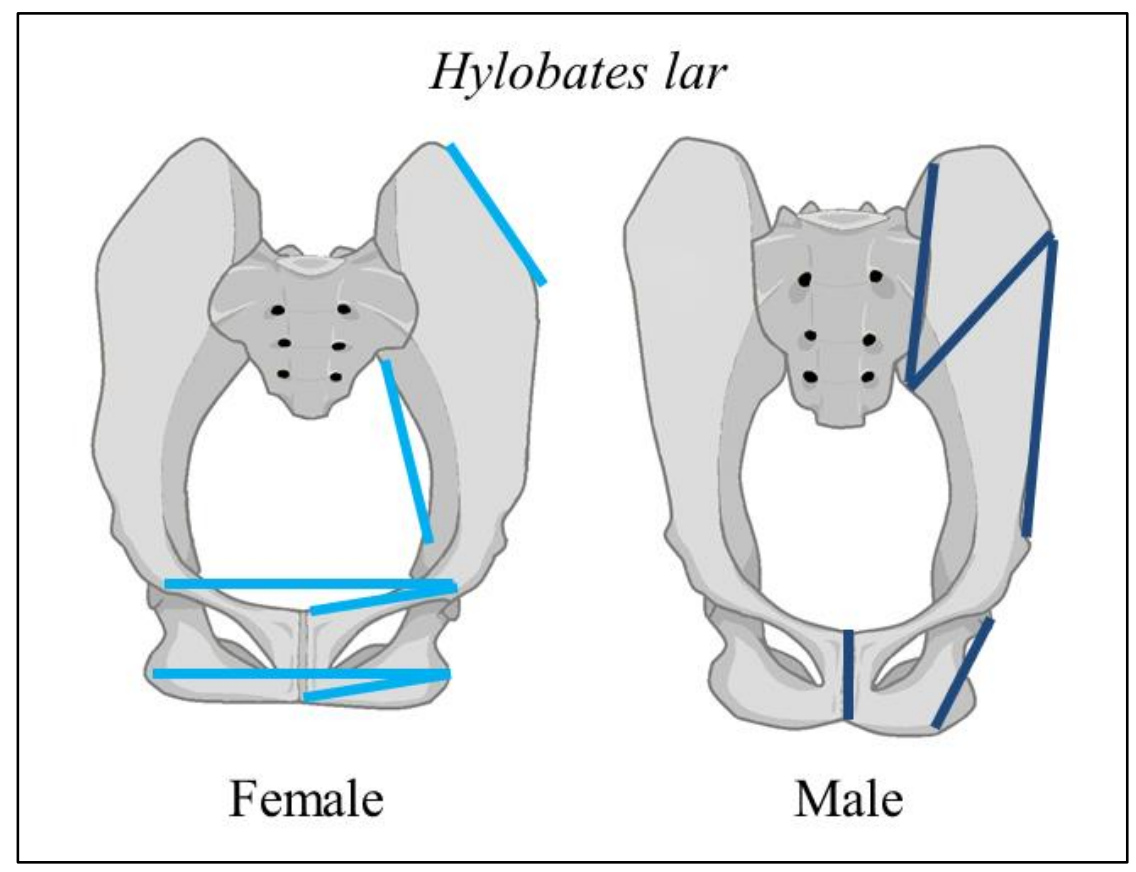

Figure 4.4b:

FORM procedure results to obtain dimorphic linear distances in the pelvis of Hylobates lar when pelves are scaled to each individual's geometric mean (body size proxy). Light blue represents linear distances which are greater in females than males. Dark blue represents linear distances which are relatively greater in males compared to females. Only non-overlapping distances are included. 


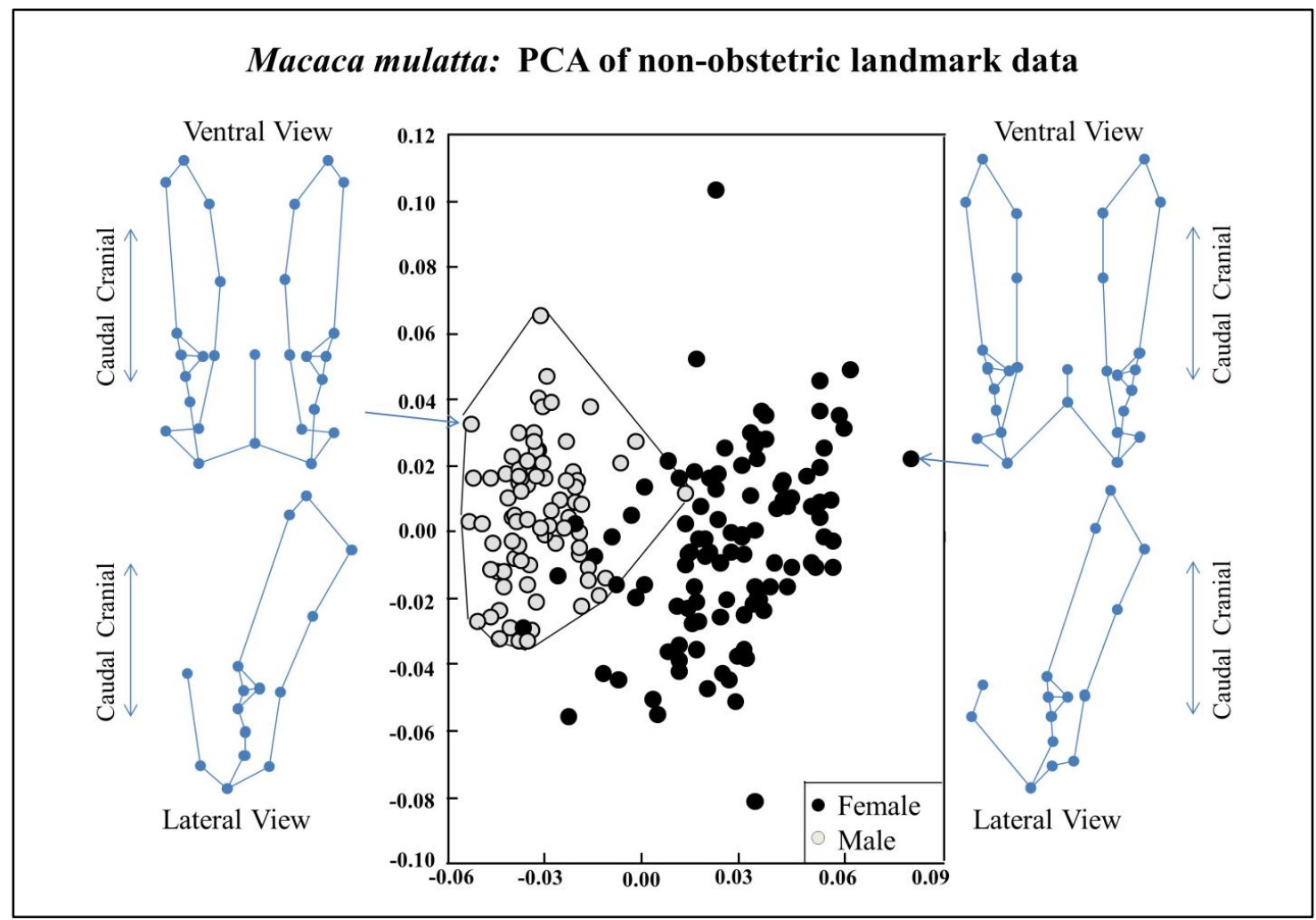

Figure 4.5a: Shape analysis for the false pelvis of Macaca mulatta. (a) Principal components analysis of false pelvis landmark data. Note the separate clustering of males and females along the $\mathrm{X}$-axis (PC1).

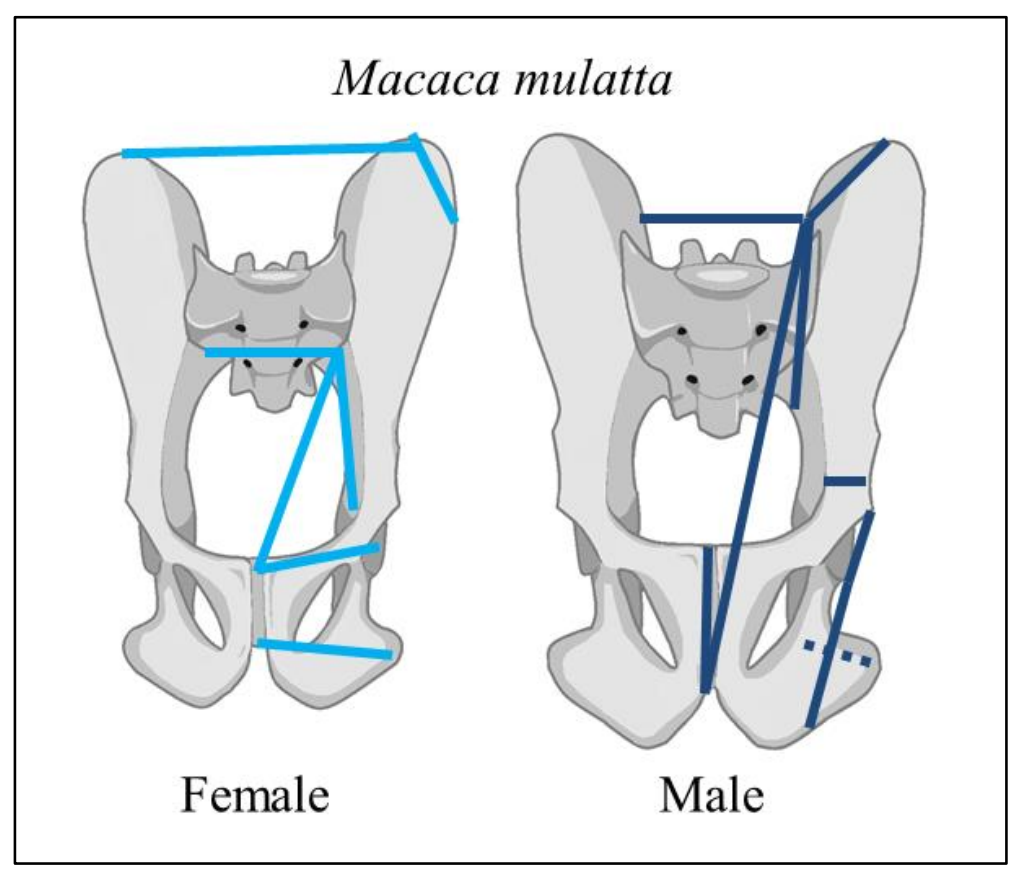

Figure 4.5b: FORM

procedure results to obtain dimorphic linear distances in the pelvis of Macaca mulatta when pelves are scaled to each individual's geometric mean (body size proxy). Light blue represents linear distances which are greater in females than males. Dark blue represents linear distances which are relatively greater in males compared to females. Only non-

overlapping distances are included. 


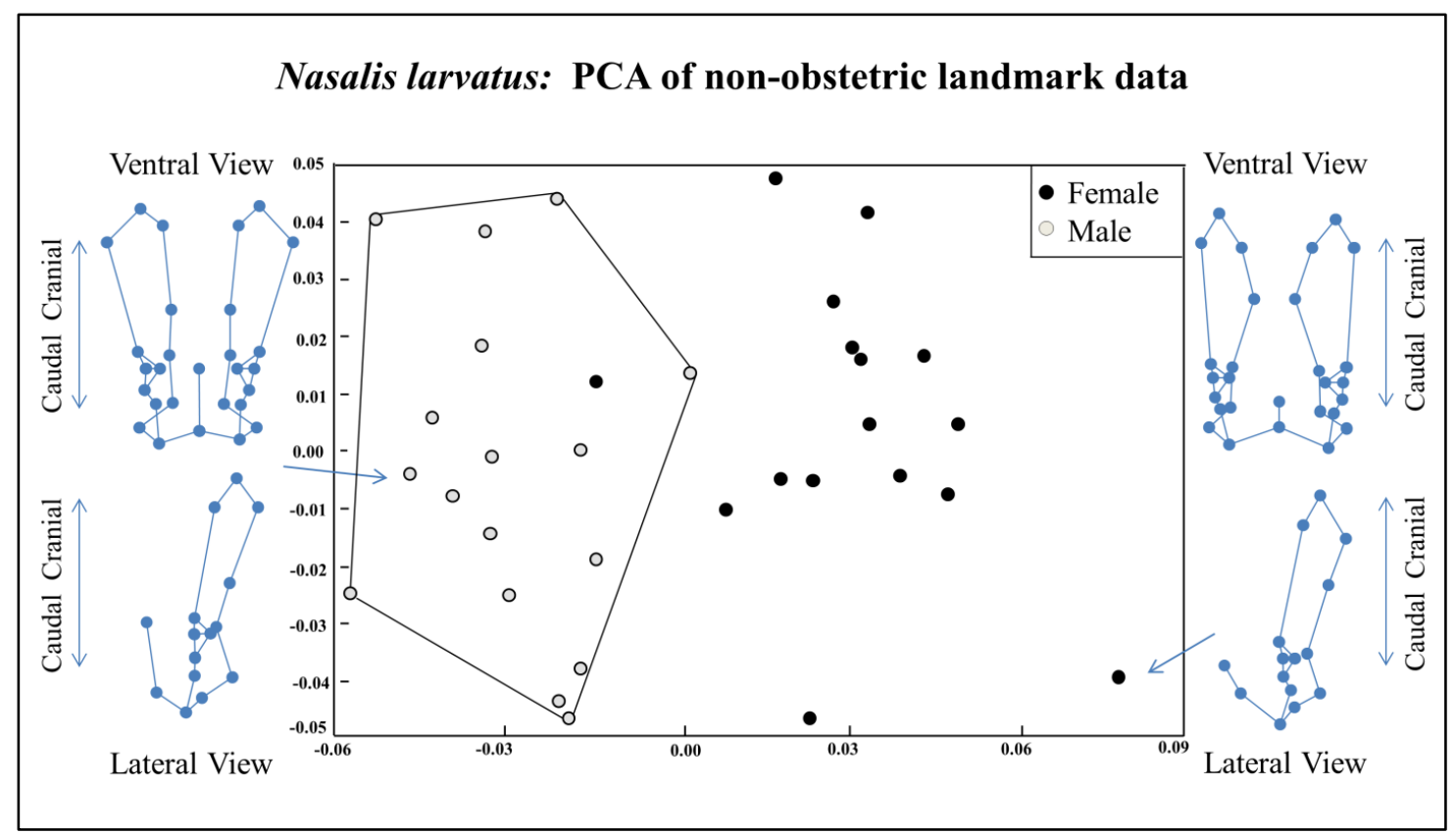

Figure 4.6a: Shape analysis for the false pelvis of Nasalis larvatus. (a) Principal components analysis of false pelvis landmark data. Note the separate clustering of males and females along the $\mathrm{X}$-axis (PC1).

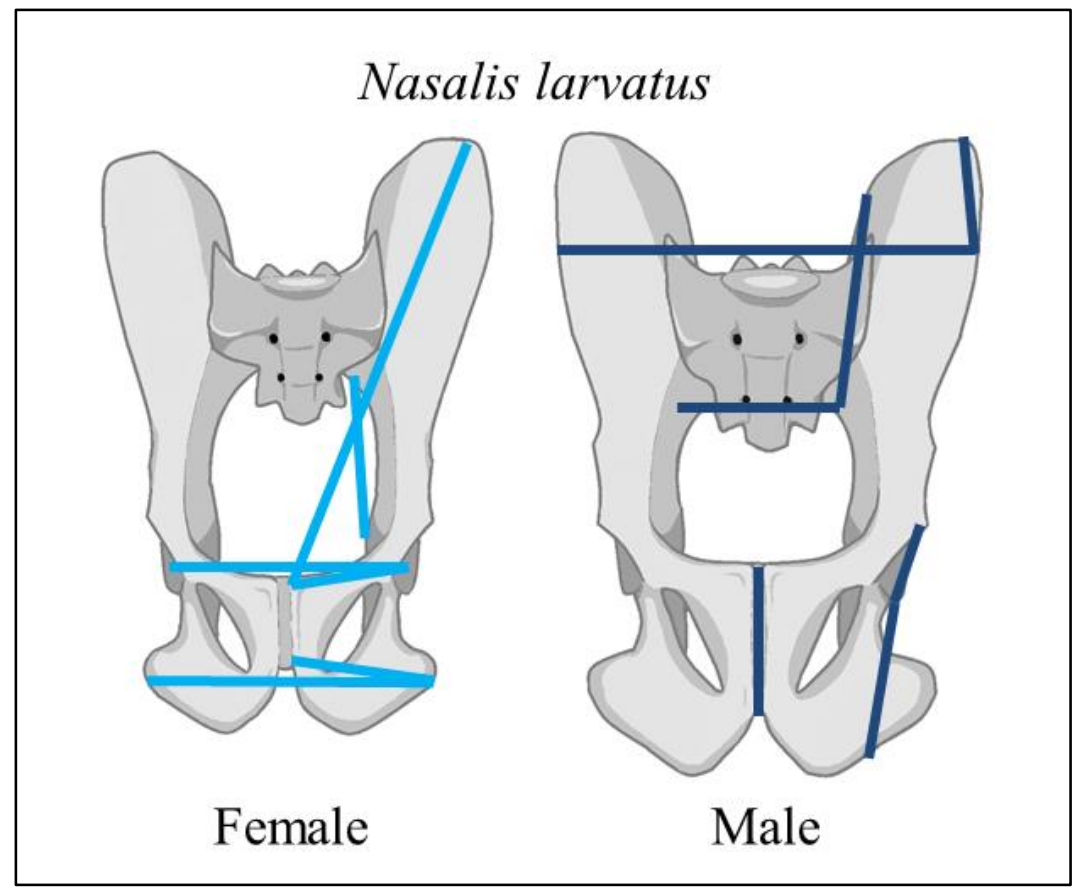

Figure 4.6b: FORM procedure results to obtain dimorphic linear distances in the pelvis of Nasalis larvatus when pelves are scaled to each individual's geometric mean (body size proxy). Light blue represents linear distances which are greater in females than males. Dark blue represents linear distances which are relatively greater in males compared to females. Only non-overlapping distances are included. 


\section{Papio anubis: PCA of non-obstetric landmark data}

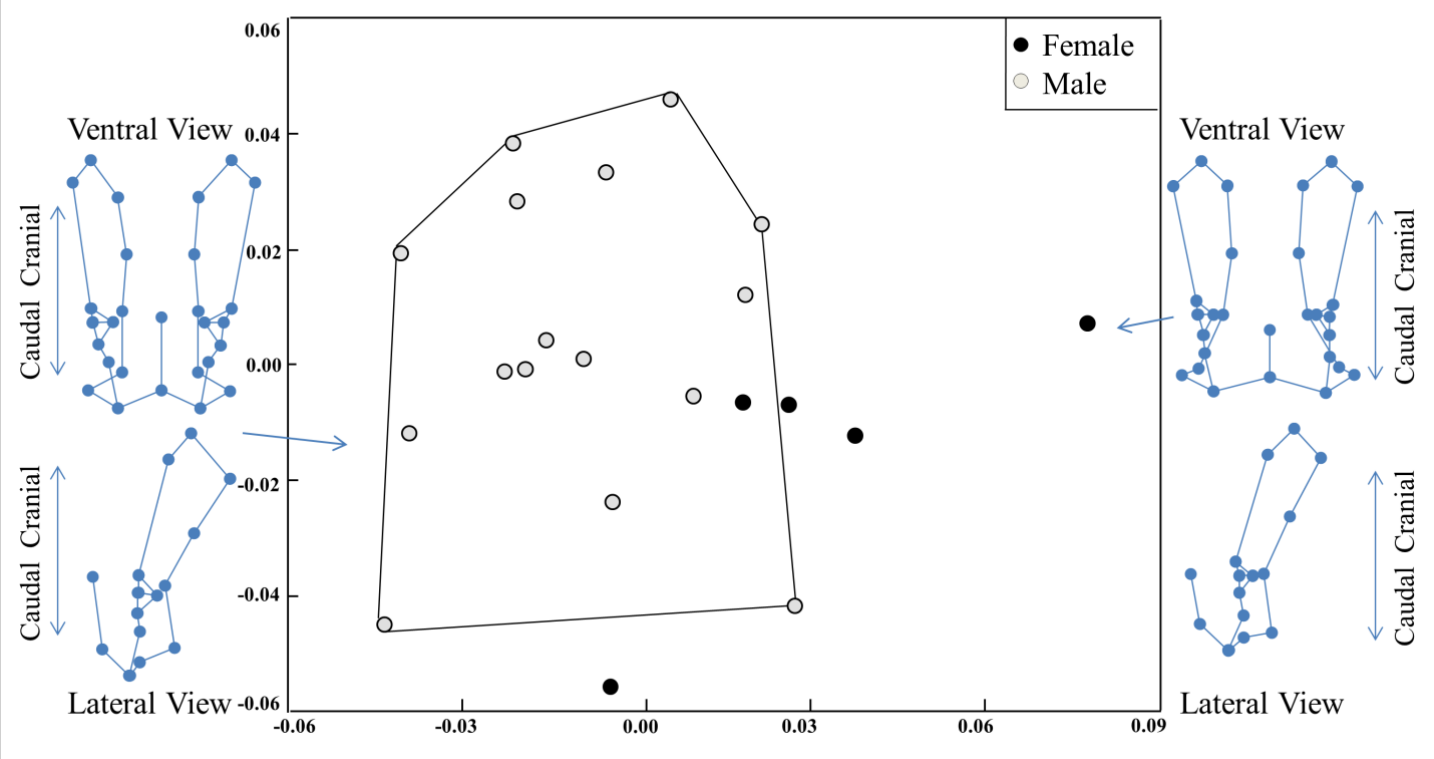

Figure 4.7a: Shape analysis for the false pelvis of Papio anubis. (a) Principal components analysis of false pelvis landmark data. Note the separate clustering of males and females along the $\mathrm{X}$-axis (PC1).

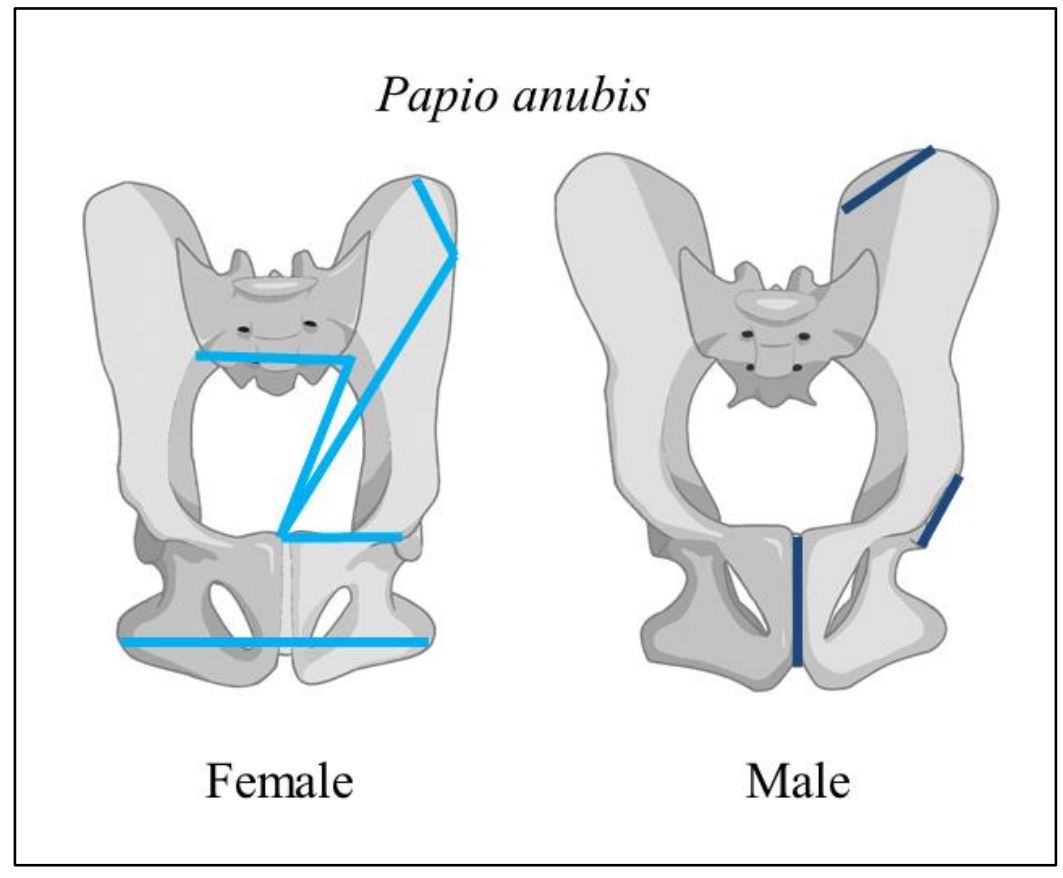

Figure 4.7b: FORM procedure results to obtain dimorphic linear distances in the pelvis of Papio anubis when pelves are scaled to each individual's geometric mean (body size proxy). Light blue represents linear distances which are greater in females than males. Dark blue represents linear distances which are relatively greater in males compared to females. Only non-overlapping distances are included. 


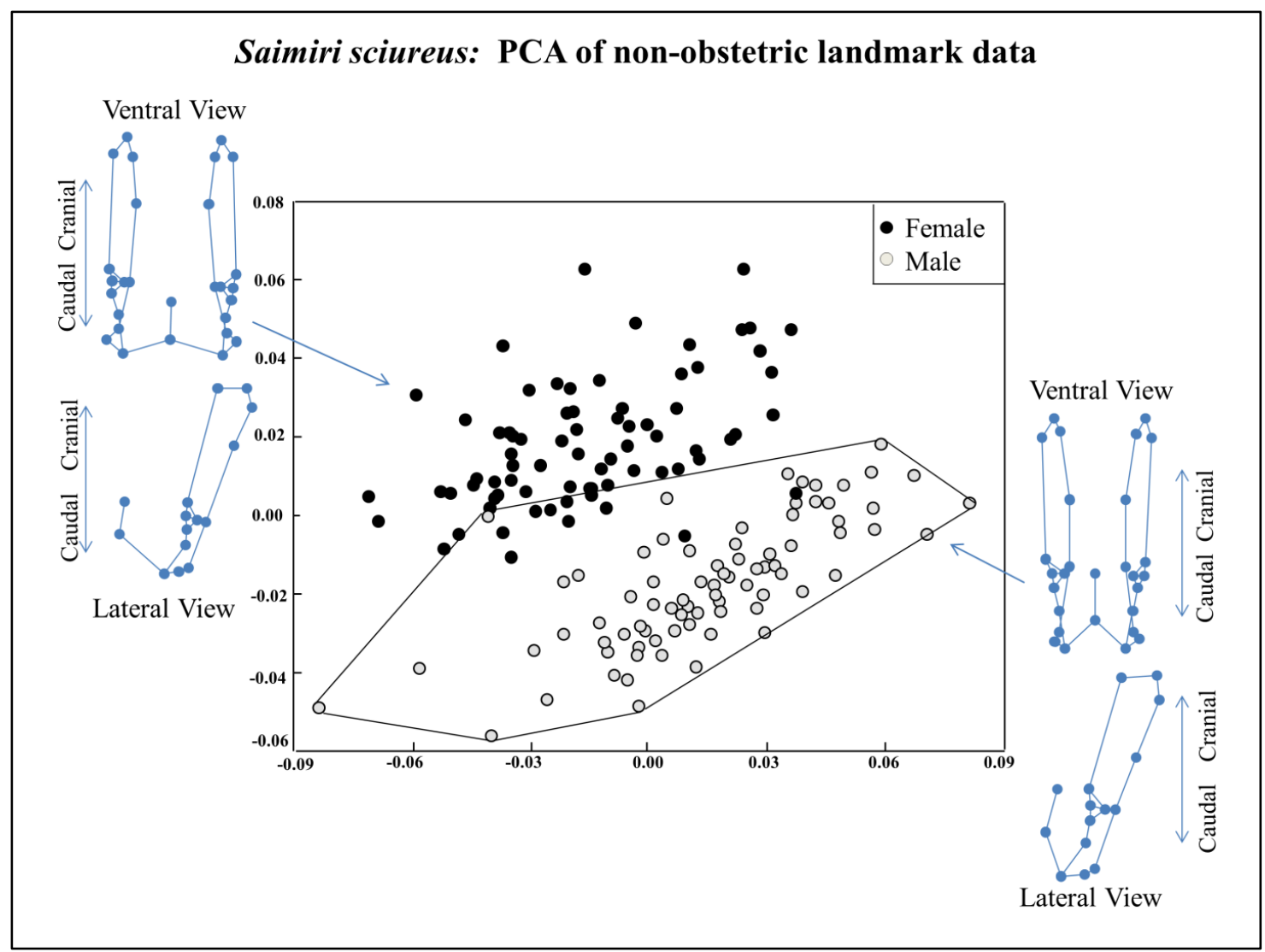

Figure 4.8a: Shape analysis for the false pelvis of Saimiri sciureus. (a) Principal components analysis of false pelvis landmark data. Note the separate clustering of males and females along PC1 and PC2.

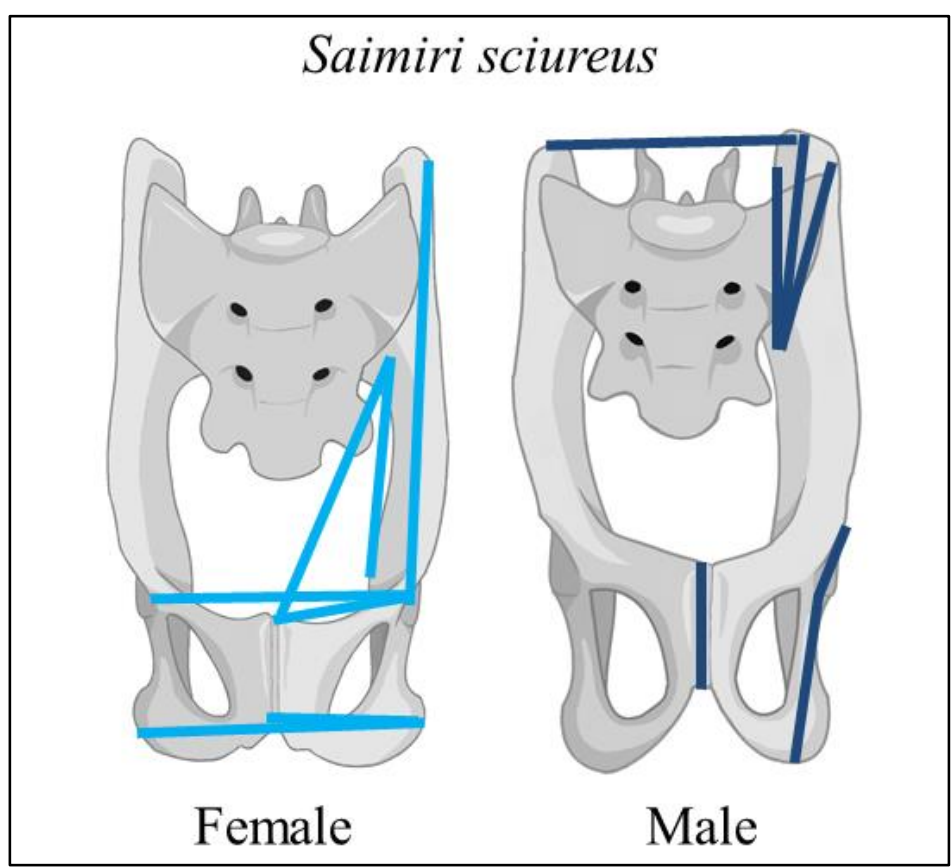

Figure 4.8b: FORM procedure results to obtain dimorphic linear distances in the pelvis of Saimiri sciureus when pelves are scaled to each individual's geometric mean (body size proxy). Light blue represents linear distances which are greater in females than males. Dark blue represents linear distances which are relatively greater in males compared to females.

Only non-overlapping distances are included. 


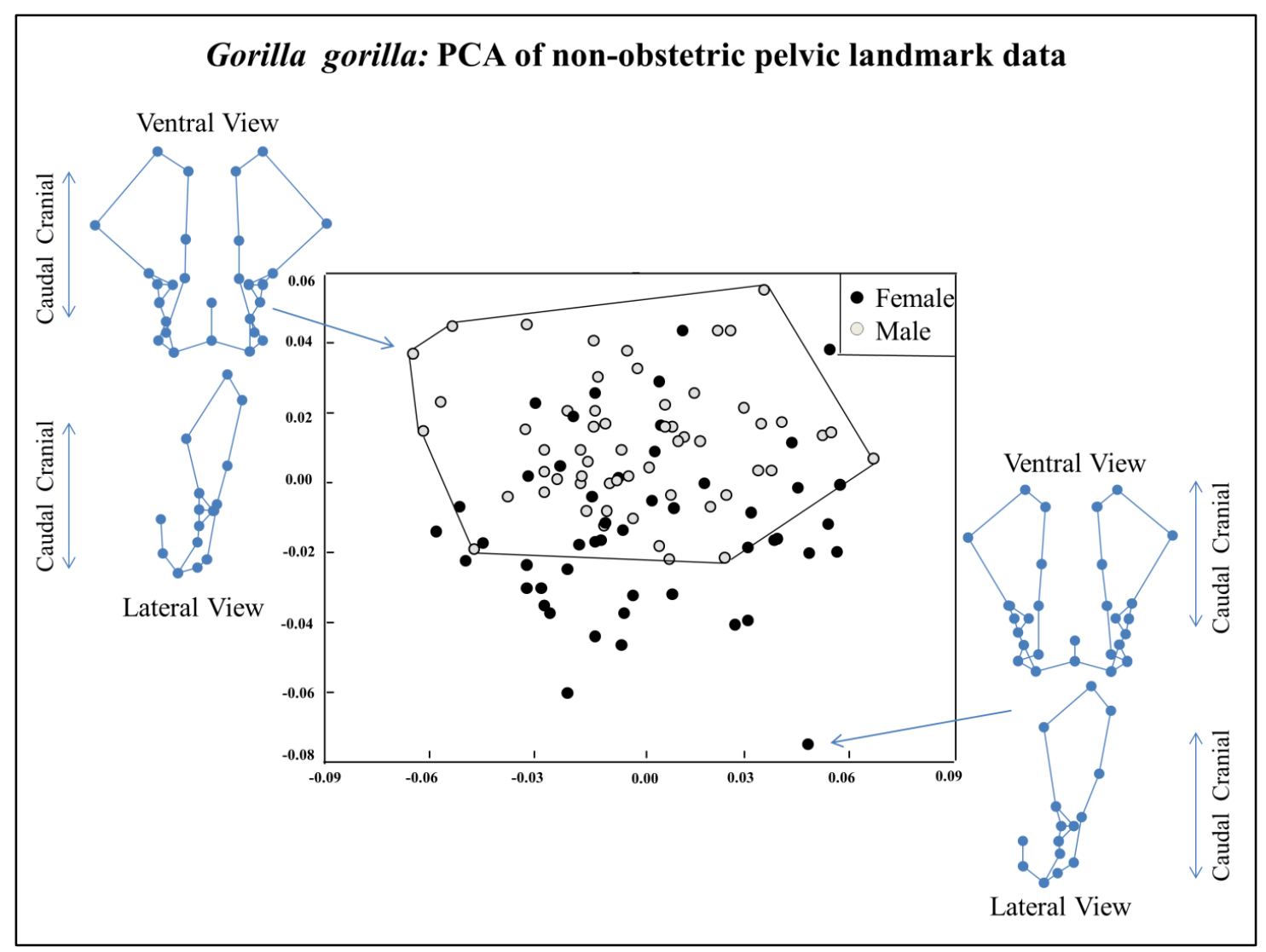

Figure 4.9a: Shape analyses for the false pelvis of Gorilla gorilla. (a) Principal components analysis of false pelvis landmark data. Note the lack of clustering of males and females along PC1 and PC2.

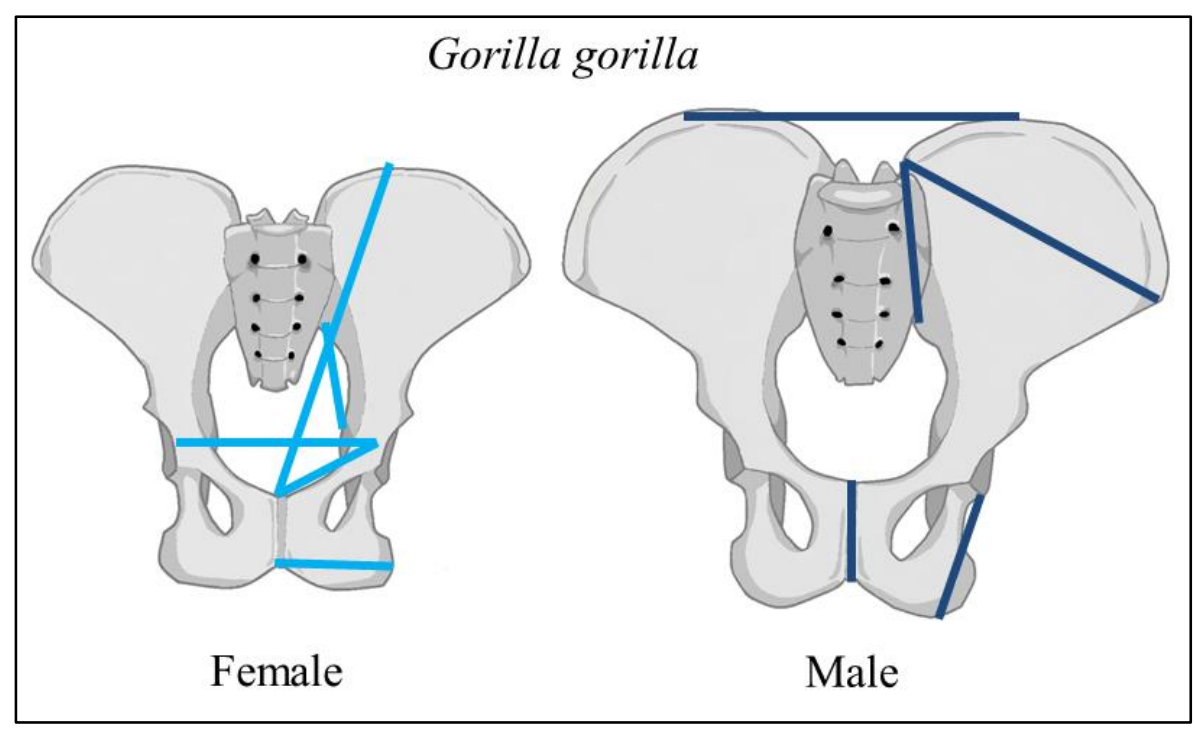

Figure 4.9b: FORM procedure results in Gorilla gorilla when pelves are scaled to geometric mean. Light blue represents distances relatively greater in females. Dark blue represents distances which are relatively greater in males. Only non-overlapping distances are included. 


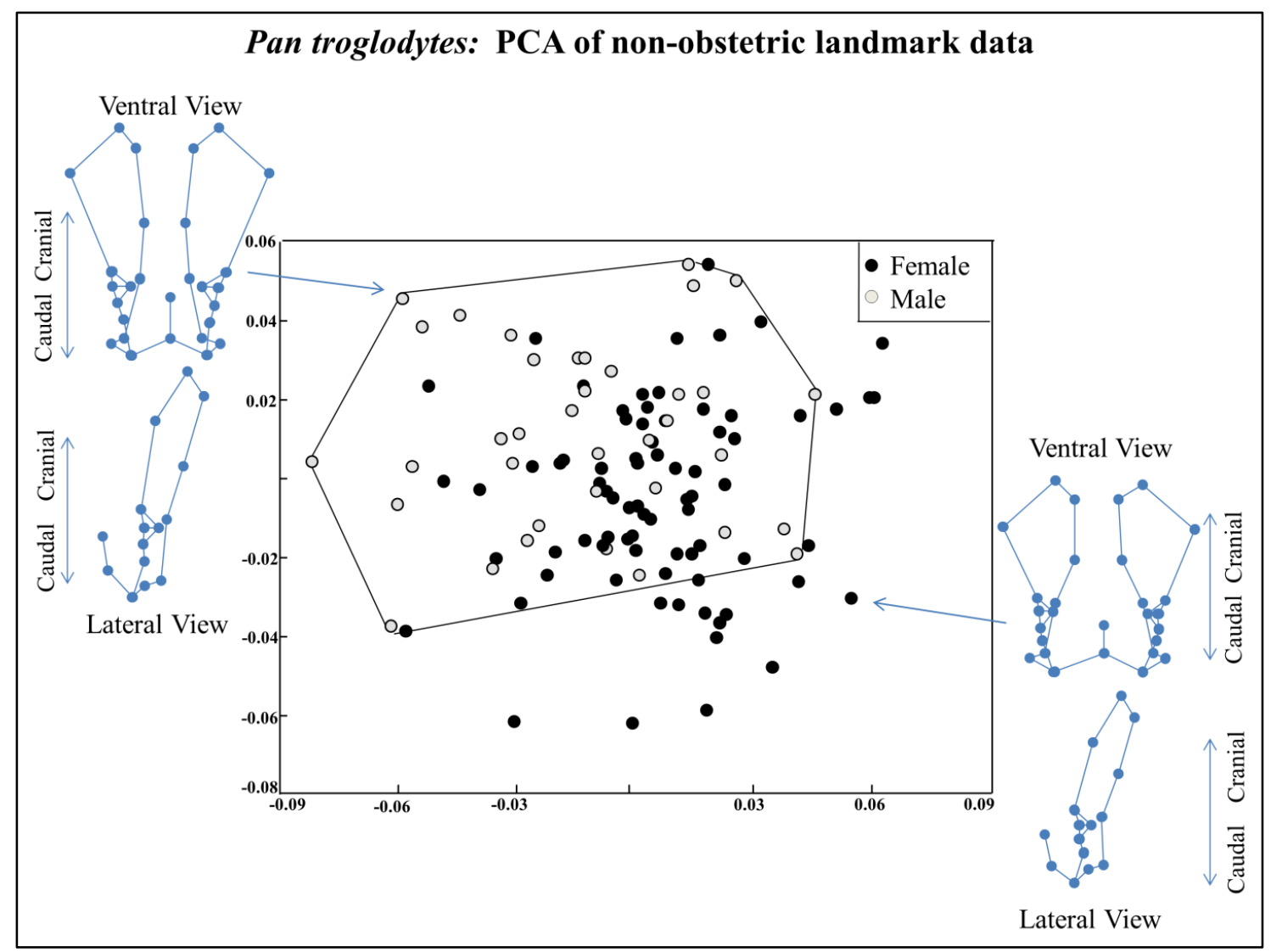

Figure 4.10a: Shape analyses for the false pelvis of Pan troglodytes. (a) Principal components analysis of false pelvis landmark data. Note the clustering of males and females along PC1 and PC2.

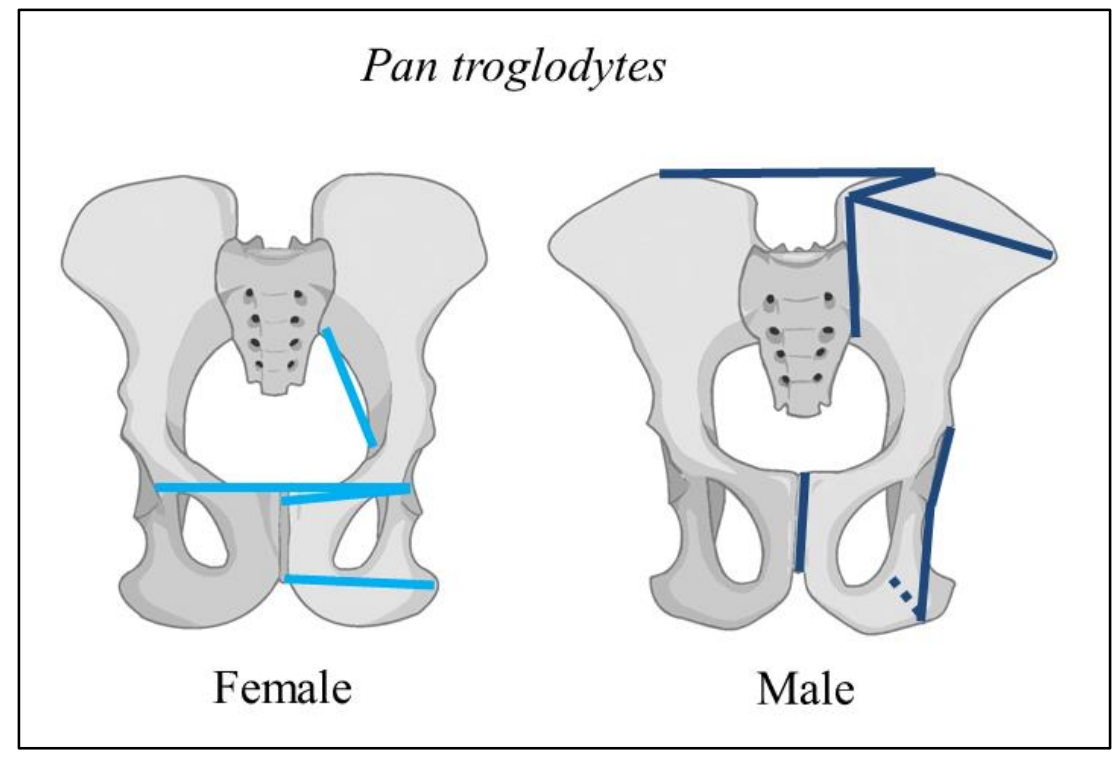

Figure 4.10b: FORM procedure results in Pan troglodytes when pelves are scaled to geometric mean.

Light blue represents distances which are relatively greater in females.

Dark blue represents distances which are relatively greater in males.

Only non-overlapping distances are included. 


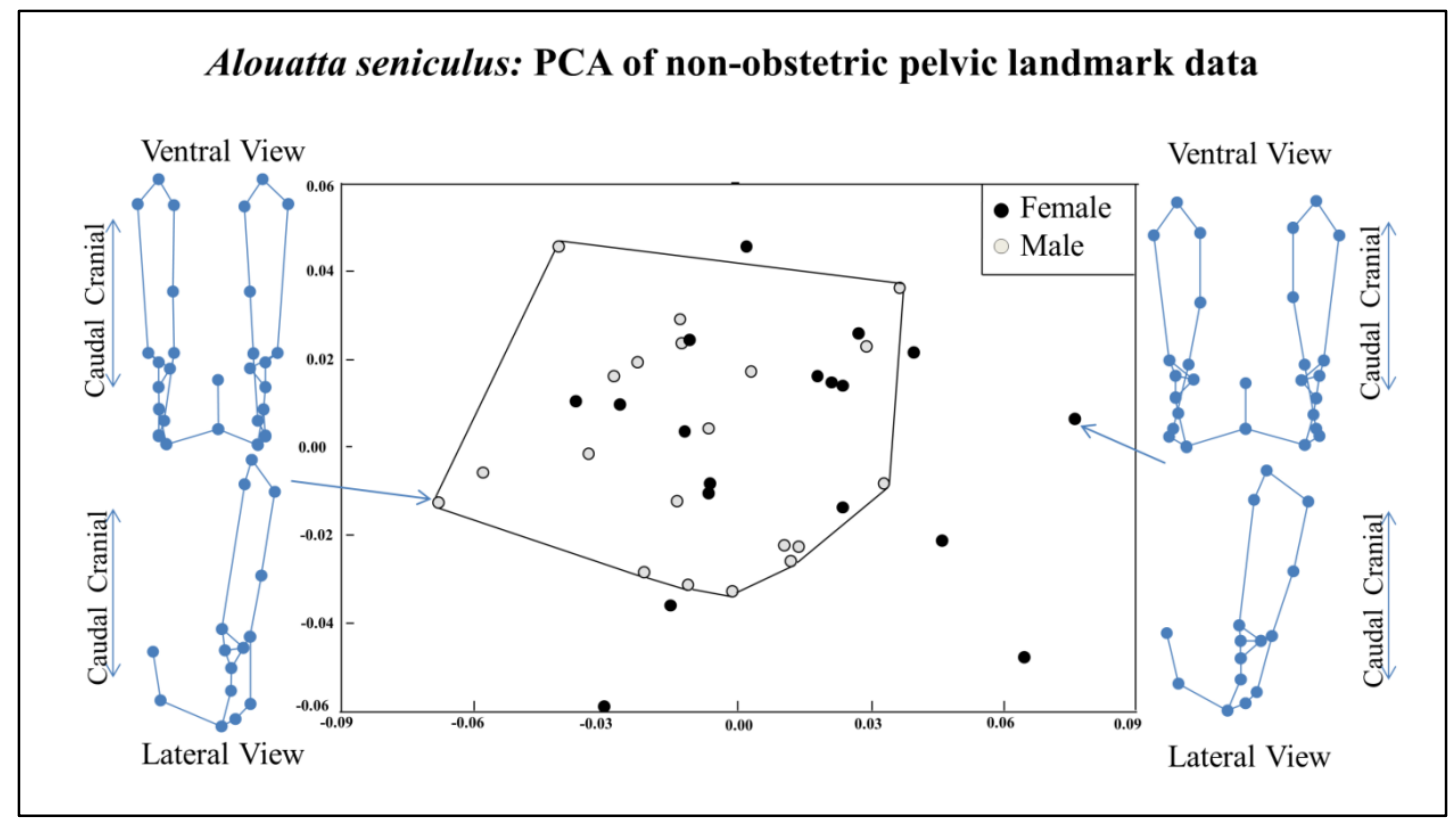

Figure 4.11a: Shape analyses for the false pelvis of Alouatta seniculus. (a) Principal components analysis of false pelvis landmark data. Note the lack of clustering of males and females along PC1 and PC2.

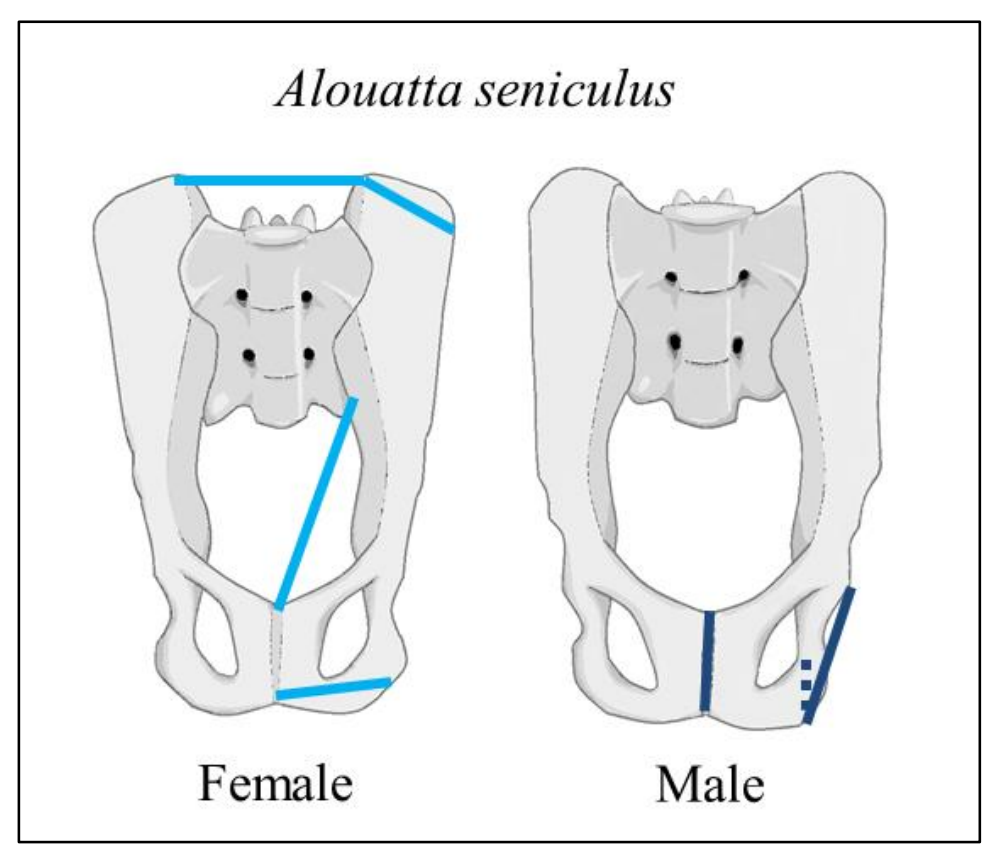

Figure 4.11b: FORM procedure results in Alouatta seniculus when pelves are scaled to geometric mean. Light blue represents distances which are relatively greater in females. Dark blue represents distances which are relatively greater in males. Only nonoverlapping distances are included. 


\section{CHAPTER 5: INTEGRATION IN THE ANTHROPOID BIRTH CANAL AND NON-OBSTETRIC PELVIS}

\section{BACKGROUND}

Morphological integration, covariation between phenotypic characters (Olsen and Miller, 1958; Chernoff and Magwene, 1999), is influenced by natural selection (Cheverud, 1981). Obstetric (birth-related) selection, which immediately affects maternal and offspring fitness, should affect morphological integration in primate pelves (Grabowski et al., 2011; Lewton, 2012; Grabowski, 2013) because all primates give birth through the bony birth canal. There is a range of cephalopelvic proportions (the fit between maternal birth canal and neonatal head) across anthropoids (Shultz, 1949; Leutenegger, 1970; Stoller, 1995), and primates that give birth to relatively large neonates are under more rigorous obstetric selection than individuals from taxa which give birth to relatively small neonates (Schultz, 1949; Leutenegger, 1970; Stoller, 1995). Consequently, there is more dimorphism in the pelves of primates that give birth to relatively large neonates compared to those that give birth to relatively small ones (See chapters 2-4) - birth canal size and shape is highly dimorphic in these species, and some parts of the non-obstetric pelvis (including biacetabular breadth and lower iliac height) are as well. This indicates that obstetric selection could also affect which parts of the pelvis are integrated (e.g., dimensions of the birth canal) as well as the degree to which they are integrated. The variation in cephalopelvic proportions in anthropoids 
provides an opportunity to quantify the effects of obstetric selection on pelvic integration.

Selection for obstetric sufficiency may shape pelvic integration in primates in several ways. First, such selection could determine how the pelvis is divided up into separate modules. (i.e., areas which have strong integration within themselves but low integration between themselves and other such modules; Klingenberg, 2008). Second, obstetric selection could determine how tightly morphologies covary (the magnitude of integration) within these individual modules. Third, obstetric selection could determine the patterns of integration (which specific parts strongly covary) within these separate modules. It should be noted that these phenomena are not mutually exclusive, and it is possible for obstetric selection to shape integration in all of these ways simultaneously. Moreover, the modular organization of the pelvis by definition results from the patterns and magnitudes of integration within modules.

A "module" is a unit or number of units of a phenotype which: 1) collectively serve a primary functional role; 2) are integrated; and 3) are somewhat independent (uncorrelated) of other such units (Cheverud, 1988, 1996; Wagner, 1996; Klingenberg, 2008). If only some parts of the pelvis are subject to selection for obstetric selection (e.g., the birth canal; see Chapter 2), whereas other parts of the pelvis are not under direct selection for birth (e.g., the non-obstetric pelvis; see Chapter 4) this could lead to the formation of separate pelvic modules (Grabowski et al., 2011; Lewton, 2012), or it could lead to a reorganization of existing modules into a new modular pattern. Results from Chapters 2 and 4 indicate that while obstetric selection does influence the size and shape of the birth canal, such selection only affects isolated parts of the non-obstetric 
pelvis (including biacetabular breadth and lower iliac height), indicating that selection on the non-obstetric pelvis might be somewhat (but not entirely) independent from the birth canal. Indeed, some previous research has indicated that the ischiopubis (which participates heavily in the birth canal) may be in a separate module than the upper ilium (Lewton, 2012), and this could indicate that the birth canal and non-obstetric pelvis may be separate modules in primates.

Previous research has indicated that humans, who have large cephalopelvic proportions and more difficult birth compared to other apes (Schultz, 1949), also have reduced covariation between the birth canal and non-obstetric pelvis compared to other apes (Grabowski, 2013). If the formation of these separate modules (i.e., birth canal and non-obstetric pelvis) in humans (compared to other great apes) is only the result of obstetric demand, other primates with large cephalopelvic proportions (e.g., smallbodied monkeys) should also exhibit low covariation between the birth canal and nonobstetric pelvis. However, this should not be the case in species with small cephalopelvic proportions, which theoretically do not have antagonistic selection pressures on the birth canal and non-obstetric pelvis. Obstetric selection should decrease the covariation between the birth canal and non-obstetric pelvis such that these are separate modules in taxa with large cephalopelvic proportions.

Selection for obstetric sufficiency may also influence how strongly traits covary (the magnitude of integration) within each of these hypothesized modules (birth canal and non-obstetric pelvis). It might be expected that taxa which give birth to relatively large neonates should have the strongest covariation between linear distances of the birth canal, because there should be strong stabilizing selection on birth canal size and 
shape in these species. Indeed, humans have higher magnitudes of integration in the birth canal compared to other apes (Grabowski et al., 2011), but it is unknown whether this is also the case in other species with large cephalopelvic proportions.

Similarly, taxa with large cephalopelvic proportions might also be expected to have higher magnitudes of integration between linear distances in the non-obstetric pelvis. Chapter 4 demonstrates that selection for obstetric sufficiency increases biacetabular breadth and lower iliac height (distance between the acetabulum and sacroiliac joints) because increasing these linear distances increases birth canal size. Any variation in linear distances of the non-obstetric pelvis could thus affect the size of the birth canal (see Chapter 4). Therefore, it might be expected that species with large cephalopelvic proportions have higher magnitudes of integration among linear distances of the non-obstetric pelvis.

Additionally, in species with large cephalopelvic proportions, it might be expected that females should have higher magnitudes of integration within the birth canal and non-obstetric pelvis compared to males (because females are under direct selection for obstetric sufficiency, but males are not). In contrast, in taxa that give birth to relatively small neonates, females should not have higher magnitudes of integration than males because these females are not subject to rigorous obstetric selection. This would also help to explain why there is a high magnitude of dimorphism in the birth canals and non-obstetric pelves of anthropoids with large cephalopelvic proportions (see Chapters 2-4) - higher integration in females would constrain female pelvic morphology compared to that of males. In summary, in species with large cephalopelvic proportions, females should have greater magnitudes of integration in the birth canal 
and in the non-obstetric pelvis compared to males, but this should not be the case in species with small cephalopelvic proportions.

A final way that obstetric selection could influence pelvic integration is by modifying the patterns of integration (which traits strongly covary within modules) in the birth canals and non-obstetric pelves of anthropoids. However, patterns of morphological integration in the primate skeleton tend to be conserved (Cheverud, 1996; Ackerman and Cheverud, 2000; Marroig and Cheverud, 2001). Therefore, it would be unlikely that obstetric selection modifies integration patterns within the birth canal or non-obstetric pelvis, but it is still possible. For example, it could be the case that taxa with large cephalopelvic proportions have strong integration between the mediolateral dimensions and circumferences of the pelvic inlet, midplane, and outlet (which are under strong stabilizing selection for obstetric sufficiency; see Chapter 2) but weaker integration between the rostrocaudal dimensions of the birth canal, which are under less stringent selection for obstetric sufficiency (see Chapter 2). However, this pattern would not be expected to be shared with taxa with small cephalopelvic proportions, because these smaller proportioned taxa are not subject to the same obstetric selection pressures.

Similar to what was proposed for magnitudes of integration, it might be expected that patterns of integration within modules could differ between males and females within large cephalopelvic proportion taxa (such that the sexes have different integration patterns due to obstetric selection on females), but again this would be unlikely considering that integration patterns within modules tend to remain conserved 
in primates (Cheverud, 1996; Ackerman and Cheverud, 2000; Marroig and Cheverud, 2001).

The goal of this study is to quantify the effects of cephalopelvic proportions on pelvic modularity, as well as to test whether obstetric demand influences the magnitudes or patterns of integration within these hypothesized modules - the birth canal or non-obstetric pelves - of anthropoid primates. This chapter tests the following hypotheses:

\section{HYPOTHESES}

Hypothesis 1: (Table 5.1) The birth canal and non-obstetric pelvis represent two separate modules.

Prediction 1a: Large cephalopelvic proportions result in low covariation between the birth canal and non-obstetric pelvis. Species with large cephalopelvic proportions will exhibit high covariation within those modules but low covariation between separate modules. However, this pattern will not be apparent in species with small cephalopelvic proportions.

Hypothesis 2: (Table 5.1) Large cephalopelvic proportions result in higher magnitudes of integration within the birth canal.

Prediction 2a: Primates with large cephalopelvic proportions will exhibit greater magnitudes of integration (i.e., greater covariance 
between linear distances) in the birth canal, due to obstetric selection maintaining pelvic morphology, compared to species with small cephalopelvic proportions.

Prediction 2b: In species with large cephalopelvic proportions females will have a greater magnitudes of integration in the birth canal compared to males, but this will not be true in species with small proportions.

Hypothesis 3: (Table 5.1) Large cephalopelvic proportions result in higher magnitudes of integration within the non-obstetric pelvis (because selection for birth sufficiency might also select for strong covariation between specific morphologies of the non-obstetric pelvis).

Prediction 3a: Primates with large cephalopelvic proportions will exhibit greater magnitudes of integration (i.e., greater covariance between linear distances) in the non-obstetric pelvis compared to species with small cephalopelvic proportions.

Prediction 3b: If prediction 3a is not supported, in species with large cephalopelvic proportions females will have greater magnitudes of integration in the non-obstetric pelvis compared to males, but in species with small cephalopelvic proportions females will not have higher integration than males.

Hypothesis 4: (Table 5.1) Large cephalopelvic proportions are not associated with specific patterns of integration in the birth canal. 
Prediction 4a: All species share similar patterns of integration in the birth canal regardless of cephalopelvic proportions.

Prediction 4b: Within species, males and females will have similar patterns of integration within the birth canal.

Hypothesis 5: (Table 5.1) Large cephalopelvic proportions are not associated with specific patterns of integration in the non-obstetric pelvis.

Prediction 5a: All species share similar patterns of integration in the non-obstetric pelvis regardless of cephalopelvic proportions.

Prediction 5b: Within species, males and females will have similar patterns of integration within the non-obstetric pelvis.

\section{MATERIALS AND METHODS}

\section{a. Samples}

Previous studies have determined that integration analyses require 40-60 individuals per species per sex for sufficient power to determine differences in integration between samples (Cheverud, 1988; Grabowski et al., 2011; Grabowski, 2013). Sufficiently large samples existed for six taxa (see also Chapter 2, Table 2.1), including four with large cephalopelvic proportions [Homo sapiens $(\mathrm{n}=156)$, Hylobates $\operatorname{lar}(\mathrm{n}=112)$, Macaca mulatta $(\mathrm{n}=187)$, Saimiri sciureus $(\mathrm{n}=153)]$, and two with small proportions [Gorilla gorilla $(\mathrm{n}=106)$ and Pan troglodytes $(\mathrm{n}=114)]$. Cephalopelvic 
proportions for species included in the present study have been presented previously (Chapter 2).

It is likely that phylogeny may influence pelvic integration, but the small number of taxa included in this study preclude formal phylogenetic testing - though techniques are available, statistical analysis would have low power to detect phylogenetic signal in the small number of taxa included (Blomberg et al. 2003). However, the sample in the present study included apes (Homo, Hylobates, Gorilla, Pan), as well as a species of Old World Monkey (Macaca mulatta) and a species of New World Monkey (Saimiri sciureus). Furthermore, this sample comprised the largest sample sizes currently in existence worldwide for most taxa represented. Moreover, the sample included large sample sizes of both monkeys and hominoids, as well as increased sample sizes of individual monkey taxa compared to previous studies (Lewton, 2012; Grabowski, 2013).

\section{b. Data}

Three-dimensional landmark data collected on the pelves of adult male and female pelves (collection techniques presented in Chapter 2), were used for these analyses (Figure 5.1, Table 5.1). All statistical procedures were performed on unilateral landmarks in these integration analyses in order to avoid spurious findings of increased magnitudes of integration due to high covariation among bilaterally-distributed landmarks (Chapters 2 and 4 included bilateral landmarks). 
For some integration analyses it was necessary to use linear distances within the birth canal and within the non-obstetric pelvis to determine the magnitude and pattern of integration. Therefore, in addition to using landmark data from the birth canal and non-obstetric pelvis, linear distances which reflect important obstetric or biomechanically-relevant dimensions in the pelvis (as described in Chapters 2 and 4) were also included. These distances were derived from 3D landmark data (Figures 5.2 and 5.3). Linear distances for the birth canal ( $\mathrm{n}=8$; Figure 5.2) include the anteroposterior and mediolateral dimensions of the pelvic inlet, midplane, and outlet, as well as sacral breadth at linea terminalis and pubis length. Linear distances chosen to represent the non-obstetric pelvis ( $\mathrm{n}=7$; Figure 5.3) included inferior (lower) iliac height, superior (upper) iliac height, iliac breadth, iliac neck thickness, ischial length, ischial tuberosity breadth, and biacetabular breadth.

\section{c. Testing Hypothesis 1}

The first hypothesis (Table 5.1) predicts that the birth canal and non-obstetric pelvis represent two separate modules. Modularity testing of 3D landmarks (Klingenberg, 2009) was used to test this hypothesis. Modularity testing uses the $\mathrm{R}_{\mathrm{V}}$ coefficient (a measure of covariation between the sets of landmarks in separate modules) to test modularity statistically, with significance of each modularity test determined using a permutation test of randomly-generated modules $(10,000$ permutations). For each species, the $\mathrm{R}_{\mathrm{V}}$ coefficient for the hypothesized modules (i.e., the birth canal and non-obstetric pelvis) was compared to the distribution of $R_{V}$ 
coefficients derived for all randomly-generated modules. If the $\mathrm{R}_{\mathrm{V}}$ coefficient for the hypothesized modules was smaller than $95 \%$ of other randomly-generated modules, the test was considered significant (i.e., that there is low correlation between modules). A significant result for modularity testing indicated that the partitioning of hypothesized modules (birth canal and non-obstetric pelvis) was supported (and that there was low correlation between modules). All modularity tests were conducted in MorphoJ (Klingenberg, 2009). This study included a much larger number of specimens for individual taxa and similar numbers of landmarks $(n=22)$ compared to a previous study of pelvic modularity (Lewton, 2012; 17 landmarks), which indicated that the power should be relatively high for the current analyses for detecting covariation between hypothesized modules.

If the birth canal and non-obstetric pelvis do, in fact, represent two modules that can respond separately to selection pressures, results for modularity testing should indicate a significant result supporting the presence of these two hypothesized modules.

\section{d. Testing Hypothesis 2}

Prediction 2a (Table 5.1) is that primates with large cephalopelvic proportions will exhibit an increased magnitude of integration in the birth canal compared to species with small cephalopelvic proportions. Therefore, in order to test this hypothesis, the magnitude of integration among linear birth canal dimensions was first calculated (Figure 5.2). 
Covariance matrices for birth canal linear metric data were calculated using $\mathrm{R}$.

In order to be able to compare statistics calculated using multiple covariance matrices, these matrices must be standardized; here, covariance matrices were standardized by the individual linear distance means within the matrices (Houle, 1992; Hansen and Houle, 2008) prior to further analysis. In order to control for the confounding effects of geographic variation within taxa the residual covariance from MANOVAs were used, with linear distances as the dependent variables and either (a) racial origin (for humans; either black or white) or (b) subspecies designation or country of origin (if known for non-human taxa) as the dependent variables (Young et al. 2010; Grabowski, 2011; Middleton, 2015). Covariances were then pooled across populations. Similarly, differences between sexes within a single population could affect measures of integration (Marroig et al., 2009). Therefore, in all analyses that compare integration between taxa, residual covariances from MANOVAs (this time with sex as the independent variable) were pooled across sexes.

To quantify the magnitude of birth canal integration within each species, the variance of each covariation matrix's eigenvalues - i.e., eigenanalysis (Cheverud, 1983; Wagner, 1984; Cheverud, 1989) - was employed to quantify the overall level of integration within each species. The rationale for employing eigenanalysis in this context is that when linear distances strongly covary with one another, the majority of the variation resides in the first dimension of a principal components analysis, which increases the variance of the eigenvalues of the first eigenvector (Wagner, 1984; Cheverud et al., 1989; Villmoare et al., 2011; Lewton, 2012). This means that species that have high magnitudes of integration (covariation) between birth canal linear 
distances exhibit high eigenvalue variances of the first eigenvector (VE), whereas taxa with low levels of integration between linear distances exhibit low VE values.

VE values are not directly comparable between samples (Pavlicev, 2009). Therefore, for each species, the original covariation matrix was bootstrapped 1000 times to create a bootstrapped sample of VE values, and the bootstrapped VE values were standardized by the maximum possible eigenvalue variance of each matrix (standardized VE) (Pavlicev, 2009).Standardized VE values were then compared between separate samples in a pairwise fashion using a bootstrap resampling method (Manly, 1997) following Lewton (2012) and Scott (2010). Statistical significance of each interspecific pairwise comparison was assessed using a difference of means test following Lewton (2012) and Scott (2010). Each specific pairwise comparison revealed whether species have statistically different magnitudes of integration within the birth canal. All eigenanalysis-based integration analyses were performed in R using the “boot" package (Ripley, 2016). The number of linear distances included for the birth canal $(n=8)$ was very low compared to the number of individuals represented by each species (minimum $n=106$ ), and this indicates that the power for all eigenanalysis-based integration analyses should be high (Cheverud, 1988).

The hypothesis that magnitude of integration in the birth canal will be greater in species with large cephalopelvic proportions compared to species with small proportions was considered to be falsified if species having large cephalopelvic proportions did not have statistically greater mean integration (and statistically greater standardized VE values) in the birth canal compared to species with small cephalopelvic proportions. 
Prediction $2 \mathrm{~b}$ posits that among species with large cephalopelvic proportions, females will have a higher magnitude of integration within the birth canal compared to males, but this will not be the case in species with small cephalopelvic proportions. In order to test this hypothesis, standardized eigenvalue variance (Standardized VE; see description above) was calculated for each sex within each species for (a) linear distances of the birth canal and (b) linear distances of the non-obstetric pelvis and then compared between sexes using a bootstrap resampling method (Manly, 1997). For each sex, the original covariation matrix was bootstrapped 1000 times, and standardized VE was calculated from the bootstrapped sample. Statistical significance of each between sex comparison was assessed using a difference of means test following Lewton (2012) and Scott (2010). Each specific pairwise comparison demonstrated whether sexes shared different magnitudes of integration within the birth canal. All eigenanalysisbased integration analyses were performed in R using the "boot" package (Ripley, 2016).

The hypothesis that females in species with large cephalopelvic proportions have higher magnitudes of integration in the birth canal and non-obstetric pelvis compared to males was rejected if females of species with large cephalopelvic proportions do not have statistically greater magnitudes of integration in the birth canal and non-obstetric pelvis than males.

\section{e. Testing Hypothesis 3}


Prediction 3a (Table 5.1) is that primates with large cephalopelvic proportions will exhibit an increased magnitude of integration in the non-obstetric pelvis compared to species with small cephalopelvic proportions. Covariance matrices for linear metric data contributing to the non-obstetric pelvis (Figure 5.3) were calculated, and matrices were standardized (Houle, 1992; Hansen and Houle, 2008) following the procedures described above (d. Testing Hypothesis 2). As previously described for the birth canal, pooled covariances from MANOVAs were used to control for the confounding effects of geographic variation and sex.

To quantify the magnitude of non-obstetric pelvis integration within each species, eigenanalysis (Cheverud, 1983; Wagner, 1984; Cheverud, 1989) was employed as described above. In order to be able to directly compare the magnitude of integration (VE) among species, VEs of each species-specific covariation matrix were standardized (standardized VE; Pavlicev, 2009) as described above, and then standardized VE was compared across all species in a pairwise fashion using a bootstrap resampling method (Manly, 1997). Statistical significance of each interspecific pairwise comparison was assessed using a difference of means test as described above. The number of linear distances included for the non-obstetric pelvis $(n=7)$ was very low compared to the number of individuals represented by each species (minimum $\mathrm{n}=106$ ), and this indicates that the power of all eigenanalysis-based integration analyses should be high (Cheverud, 1988).

The hypothesis that magnitude of integration in the non-obstetric pelvis will be greater in species with large cephalopelvic proportions compared to species with small proportions was considered to be falsified if species having large cephalopelvic 
proportions did not have statistically greater mean integration (and statistically greater standardized VE values) in the non-obstetric pelvis compared to species with small cephalopelvic proportions.

Prediction $3 \mathrm{~b}$ further predicts that among species with large cephalopelvic proportions, females will have a higher magnitude of integration within the nonobstetric pelvis compared to males, but this will not be the case in species with small cephalopelvic proportions. In order to test this hypothesis, standardized eigenvalue variance (Standardized VE; see description above) was calculated for each sex within each species for linear distances of the non-obstetric pelvis and then compared between sexes using a bootstrap resampling method (Manly, 1997), as described above. Statistical significance of each between sex comparison was assessed using a difference of means test.

The hypothesis that females in species with large cephalopelvic proportions have higher magnitudes of integration in the non-obstetric pelvis compared to males was rejected if females of species with large cephalopelvic proportions do not have statistically greater magnitudes of integration in the non-obstetric pelvis than males.

\section{f. Testing Hypothesis 4}

Prediction 4a (Table 5.1) states that all species will exhibit similar patterns of integration within the birth canals. In order to test this hypothesis, Mantel tests of matrix similarity (Mantel, 1967) were used to compare integration patterns in the birth canal between species pairs. Mantel tests quantified the correlation between two matrices 
using a permutation approach with the null hypothesis that two matrices are completely unrelated. Thus, a significant positive result $(\mathrm{p}<0.025$ as indicated by the False Discovery Rate procedure; see section g below for justification for this alpha level) indicated that two matrices are not statistically significantly different, and that two species share a similar overall pattern of integration. Power for these Mantel tests is

high due to the large sample sizes collected for this study (Dietz, 1983).

Mantel tests of matrix similarity, which quantify whether there are statistical differences in the pattern of integration between species, do not allow interpretation of how these patterns of integration actually differ between groups (if integration patterns do differ). However, these statistics did provide information about whether patterns of pelvic integration differ between groups, which provided a set of hypotheses to test further in the future using bootstrap-based methods (Cole, 2002) to localize the sites where these patterns of integration differ.

Prediction $4 \mathrm{~b}$ posits that the patterns of integration in the birth canal are similar between sexes. Mantel tests of matrix similarity, as outlined above, were used to test whether males and females within each species differed in their overall patterns of integration in the birth canal.

\section{g. Testing Hypothesis 5}

Prediction 5a (Table 5.1) posits that all species will exhibit a similar pattern of integration within the non-obstetric pelvis. In order to test this hypothesis, Mantel tests (Mantel, 1967) of matrix similarity were used to compare integration patterns in the 
birth canal between species pairs as described above. Power for these Mantel tests should be relatively high due to the large sample sizes collected for this study (Dietz, 1983).

Prediction $5 \mathrm{~b}$ posits that the patterns of integration in the non-obstetric pelvisare similar between sexes. Mantel tests of matrix similarity were used to test this hypothesis.

\section{h. Controlling for Multiple Comparisons}

In order to control for Type I Error in this study, a False Discovery Rate (FDR) procedure (Benjamini and Hochberg, 1995) as described in Chapter 2 was performed on all raw p-values. The FDR procedure indicates that in order to maintain a false discovery rate of $5 \%, \alpha$-level should be set at 0.025 . Therefore, unadjusted p-values are reported in the text, but only those p-values less than 0.025 are considered significant.

\section{i. Limitations}

The statistics used here to quantify differences in the pattern of integration among species and between sexes (i.e., Mantel Tests of matrix similarity) did not allow interpretation of specifically how and where these patterns of integration actually differ between groups. Therefore, future research should aim to quantify the precise differences in patterns of integration between sexes and between species using bootstrap-based methods (Cole, 2002). 
The samples used here were not ideal for controlling for phylogeny, as there are only six taxa represented. However, these are the only taxa in existence with large enough sample sizes to perform integration analyses for which there are also data on cephalopelvic proportions, which is key to these analyses. Therefore, future studies should seek to quantify cephalopelvic proportions in larger numbers of taxa, as this will allow further comparison of integration patterns as well as formal phylogenetic comparisons.

\section{RESULTS}

\section{a. Hypothesis 1: Are the Birth Canal and Non-Obstetric Pelvis Separate}

\section{Modules?}

Hypothesis 1 predicts that the birth canal and non-obstetric pelvis represent two separate modules within the pelvis, and that this should be most apparent in species with large cephalopelvic proportions. This hypothesis can be rejected (Table 5.1). Modularity testing (Table 5.4) does indicate that the birth canal and non-obstetric pelvis are separate modules in two of the four taxa with large cephalopelvic proportions Homo sapiens $(\mathrm{p}=0.0005$; Table 5.4) and Macaca mulatta $(\mathrm{p}=0.0096$; Table 5.4). Support was not found for these being separate modules in any other species $(\mathrm{p}>0.025$ in all cases). It is possible that sample size affects the outcome of the observed results because positive results were obtained for the two taxa with the largest sample sizes $(H$. sapiens $\mathrm{n}=156 ;$ M. mulatta $\mathrm{n}=187$ ), but these are similar sample sizes to other taxa for which positive results were not obtained (e.g., Saimiri sciureus, n=153). Therefore, 
these results do not appear to be sample size dependent. Thus, the results of this study indicate that the birth canal and non-obstetric pelvis are separate modules in $H$. sapiens and M. mulatta, but not in other included taxa. Because low covariation between the birth canal and non-obstetric pelvis characterizes only two species with large cephalopelvic proportions, the hypothesis that large cephalopelvic proportions influence pelvic modularity (Hypothesis 1; Table 5.1) is not supported.

\section{b. Hypothesis 2: Do cephalopelvic proportions influence the magnitude of integration in the birth canal?}

Prediction 2a posits that primates with large cephalopelvic proportions exhibit an increased magnitude of integration in the birth canal compared to species having small cephalopelvic proportions. Prediction 2a is not supported (Tables 5.5 and 5.6). Overall, species with large cephalopelvic proportions do not have statistically higher magnitudes of integration in the birth canal than species with small proportions (Table 5.6). Though Hylobates lar (large proportions) does have the highest magnitude of integration in the birth canal (Standardized VE $=0.10$; Table 5.5), Gorilla gorilla (small proportions) has a higher magnitude of integration than Homo sapiens and Macaca mulatta (0.0838 in Gorilla vs. 0.0787 in both Homo and 0.0625 in Macaca; Table 5.5).

These results do not appear to be due to sample size discrepancies, because two taxa which have low magnitudes of integration $H$. sapiens and $M$. mulatta also have the highest sample sizes. Thus, taxa with large proportions do not have greater magnitudes 
of integration in the birth canal compared to small proportion species (Prediction 2a not supported).

However, if we look within species, cephalopelvic proportions do appear to increase the magnitude of integration in females compared to males (Table 5.7), supporting Prediction 2b. Across species with large cephalopelvic proportions except for Macaca mulatta, females have higher magnitudes of integration (i.e., higher standardized VE values; Table 5.7) in the birth canal than males (all p-values $<0.025$ ). This result for $M$. mulatta could be related to the fact that this sample comes from a population living in a non-native environment that may exhibit reduced genetic variability compared to wild specimens, which could affect integration in this sample (see further discussion below). This is not true for either species with small cephalopelvic proportions - in Gorilla gorilla, there is no difference between the sexes in the magnitude of integration in birth canal linear distances $(\mathrm{p}=0.672)$, while in Pan troglodytes, males actually have greater birth canal integration than females $(\mathrm{p}=0.002)$.

Because large cephalopelvic proportions are found to be related to higher magnitudes of integration in female birth canals compared to those of males in 3 of 4 species with large cephalopelvic proportions (and neither species with small proportions), Hypothesis 2 cannot be rejected.

\section{c. Hypothesis 3: Do cephalopelvic proportions influence magnitudes of integration in the non-obstetric pelvis?}


Hypothesis 3, Prediction 3a predicts that species with large cephalopelvic proportions will have higher magnitudes of integration in the non-obstetric pelvis than species with small proportions. This prediction is supported, because taxa with large cephalopelvic proportions (with the exception of Macaca mulatta) do have statistically higher magnitudes of integration (Range of 0.0599-0.0759; Table 5.9) (standardized VE) in the non-obstetric pelvis compared to all taxa with small proportions (both < 0.0595; Table 5.8 and 5.9).

Prediction $3 b$ further predicts that females among species with large cephalopelvic proportions will have greater magnitudes of integration in the nonobstetric pelvis compared to males, but this will not be the case in species with small proportions. This prediction is also supported in 3 of 4 species with large cephalopelvic proportions (Table 5.10). Within all species with large cephalopelvic proportions except Homo sapiens, females do have higher magnitudes of integration compared to males (all p-values $<0.002$; Table 5.10), but this is not the case in either species with small cephalopelvic proportions. The fact that in humans, males have statistically higher magnitudes of non-obstetric pelvic integration could reflect the fact that human female non-obstetric pelvic form is frequently argued to represent a tradeoff between obstetric and locomotor functions, whereas that of males represents selection for locomotion only, because bipeds are under selection for reduced biacetabular breadth and lower iliac height (Washburn, 1960; Pinkerton, 1973; Zihlman, 1978; Ruff, 1995), but reduction of these distances is obstetrically dangerous (Rosenberg, 1992; Ruff, 1995; Rosenberg, 2002). 


\section{d. Hypothesis 4: Do cephalopelvic proportions influence patterns of integration in the birth canal?}

Prediction 4a predicts all species share similar patterns of integration in the birth canal regardless of cephalopelvic proportions. This prediction is supported because the results of pairwise Mantel tests of matrix similarity (Table 5.11) indicate that integration patterns in the birth canal are similar among all anthropoid species included $(\mathrm{p}<0.001$ in all cases). Differences in cephalopelvic proportions across taxa do not appear to influence differences in birth canal integration patterns across species.

Similarly, males and females have similar patterns of integration in the birth canal among all taxa included (Table 5.12).

Based on the results of this study, Hypothesis 4 cannot be rejected (Table 5.1).

\section{e. Hypothesis 5: Do cephalopelvic proportions influence patterns of integration in the non-obstetric pelvis?}

Prediction 5a posits that primates have similar patterns of integration in the nonobstetric pelvis regardless of cephalopelvic proportions. This prediction is not supported (Table 5.13), because all primates do not share one pattern of integration (Table 5.13). However, patterns of integration do not appear to be associated with cephalopelvic proportions. Homo sapiens share similar integration patterns in the non-obstetric pelvis with Saimiri sciureus $(\mathrm{p}=0.010$; large proportions) and Gorilla gorilla $(\mathrm{p}=0.10$; small proportions; Table 5.13). Gorilla gorilla and Pan troglodytes (both small proportions) 
also share similar patterns of integration $(\mathrm{p}<0.001)$. Hylobates lar and Saimiri sciureus (both large proportions) do share a similar pattern of integration $(\mathrm{p}<0.001)$ in the nonobstetric pelvis, but this pattern is not shared with other species with large cephalopelvic proportions. Macaca mulatta does not share a pattern of integration with any other taxon.

Predictions $5 \mathrm{~b}$ predicts that sexes share similar patterns of integration in the nonobstetric pelvis. This was the case for most species, but not for Macaca mulatta (large cephalopelvic proportions) and Pan troglodytes (small proportions). These sex differences in the patterns of integration in the non-obstetric pelvis do not appear to be influenced by cephalopelvic proportions (Table 5.14).

Based on the results of this study, Hypothesis 5 cannot be rejected (Table 5.1) because although there are some differences among taxa in integration patterns, these patterns do not appear to be related to cephalopelvic proportions.

\section{DISCUSSION AND CONCLUSIONS}

The hypothesis that the birth canal and non-obstetric pelvis are separate modules across anthropoids was rejected for all taxa besides Homo sapiens and Macaca mulatta. This result for $H$. sapiens is consistent with previous research (Grabowski, 2013), which found humans to have less covariation between the birth canal and other pelvic traits compared to apes. There are two possibilities that may explain this increased modularity in human pelves. First, this result could reflect the fact that the birth canal and nonobstetric pelvis are hypothesized to be under antagonistic pressures of selection for 
obstetric sufficiency and selection for bipedal locomotion (termed the "obstetric dilemma"; Washburn, 1960; Pinkerton, 1973; Zihlman, 1978; Ruff, 1995; Lovejoy, 2005). This is also consistent with the fact that in H. sapiens, females have statistically greater mean integration (higher covariation) between linear distances of the birth canal, but males have greater integration than females in non-obstetric pelvic traits, a pattern not seen in any other species. If increased modularity in humans is the result of antagonistic selection pressures, it is likely that selection for obstetric sufficiency has increased covariation between linear distances in the birth canals of females (because any variation in birth canal shape could lead to cephalopelvic disproportion), but this selection pressure for birth in females simultaneously reduced integration between nonobstetric linear distances in females compared to males, who are only under direct selection for bipedal locomotion and visceral support. In this scenario, decreased covariation between the birth canal and non-obstetric pelvis is the result of increasing cephalopelvic proportions in bipedal hominins.

An alternative possibility is that greater modularity between the birth canal and non-obstetric pelvis characterized early hominins (who likely had smaller cephalopelvic proportions than modern humans; Tague and Lovejoy, 1986; Desilva, 2011) due to the adoption of a novel form of locomotion (bipedalism). Covariation between linear distances in the hominin pelvis was decreased during the transition to bipedal locomotion, and this increased pelvic modularity in hominins allowed our pelves to more easily adapt for successful birth as neonatal brain size increased later in our lineage (see also Grabowski et al., 2011). In this second scenario, the increased modularity of hominin pelves is the result of selection for bipedalism, and this increased 
modularity was the coopted for birth once neonatal (and adult) brain size began to increase.

Like humans, there is less covariation between the birth canal and non-obstetric pelvis in Macaca mulatta, but the reasons for this result are less apparent, and is likely different from the reasons that humans have increased modularity. The sample of $M$. mulatta used for this study came from a free-ranging, provisioned population from Caya Santiago, Puerto Rico (a non-native environment for this taxon, which consists of descendants of individuals transferred from India in 1938). The increased modularity seen in this sample of $M$. mulatta could be related to the fact that this population was recently removed from their natural environment and is in the process of adapting to this novel location. Alternatively, since this population of macaques is descended from a small group that was brought from India, it is possible that this population is descended from a small group that had reduced covariation between the birth canal and non-obstetric pelvis, and this group went through a genetic bottleneck event when transferred. This bottleneck hypothesis for the Cayo Santiago macaques has been supported by genetic data (Buettner-Janusch et al., 1974; Buettner-Janusch and Sockol, 1977; Duggleby et al., 1986; Kanthaswamy et al., 2016). Future research should examine the difference between pelvic modularity in this sample as compared to wild M. mulatta specimens.

It is also possible that there is a common explanation for increased modularity in both Homo sapiens and Macaca mulatta. Both humans and M. mulatta have very wide species ranges. In fact, $M$. mulatta has the largest species range of any extant nonhuman primate, with the species inhabiting a large region that spans both warm tropical 
and cold high altitude environments (Pocock, 1932; Miller, 1933). Greater phenotypic plasticity and lower genetic and developmental constraints are thought to contribute to success in novel environments (the adaptive plasticity hypothesis; Agrawal, 2001; Kawecki, 2008). Greater modularity in the pelves of these two species could reflect selection for reduced covariation due to genetic factors, and consequently greater plasticity, in these species. It should be noted that besides humans and $M$. mulatta, all other species included in this study exhibit smaller species ranges - Hylobates lar is restricted to southeast Asia (Brandon-Jones et al., 2004); Saimiri sciureus is primarily restricted to tropical regions of the Amazon basin (Boubli et al., 2008); Gorilla gorilla and Pan troglodytes are both restricted to equatorial regions of western Africa.

Unlike covariation between modules (modularity), the magnitudes of integration within both the birth canal and non-obstetric pelvis are clearly influenced by selection for obstetric sufficiency within species. Among taxa included in this study that give birth to relatively large neonates, females tend to have have higher magnitudes of integration in the birth canal (all species except $M$. mulatta) and non-obstetric pelvis (all species except $H$. sapiens) than males. This is not true in species with small cephalopelvic proportions. It is possible that this result is influenced by phylogeny - the two species included with small proportions are closely phylogenetically related (Gorilla gorilla and Pan troglodytes). Yet, Pan troglodytes is more closely related to Homo sapiens, so it appears that phylogeny does not influence sex differences in the magnitudes of integration to the extent of cephalopelvic proportions.

Thus, the results of this study do suggest that sex differences in the magnitude of integration in the birth canal and non-obstetric pelvis (such that females have greater 
integration than males) is related to large cephalopelvic proportions (Tables 5.7 and 5.10). This result is not unexpected, because females are under direct selection for obstetric sufficiency, whereas males are not. Therefore, variation in birth canal or nonobstetric size or shape will have more immediate selective consequences in females who give birth to relatively large neonates. Furthermore, this increased magnitude of integration in females compared to males (but only in species with large cephalopelvic proportions) also helps to explain why these species also maintain high levels of dimorphism in birth canal and non-obstetric pelvic form (see Chapters 2- 4). In species with large proportions, female pelvic form is more constrained compared to that of males, but this is not true in species with small proportions, which is consistent with the fact that there is typically more overlap between male and female pelvic morphology in smaller proportioned taxa (see Chapters 2 and 4).

Finally, the patterns of integration (which specific traits strongly covary) in the birth canal and non-obstetric pelvis do not appear to be influenced by cephalopelvic proportions at all (Tables 5.11- 5.14). This result is consistent with previous research which finds patterns of morphological integration in the skeleton tend to be more conserved across primates than magnitudes of integration (Cheverud, 1996; Ackerman and Cheverud, 2000; Marroig and Cheverud, 2001). Indeed, patterns of integration in the birth canal (Tables 5.11) are shared among all taxa included in this study (and between sexes in all taxa; Table 5.12). This could reflect that fact that there is strong stabilizing selection on the pattern of birth canal integration in primates because it is vital that the shapes of the birth canal planes covary in a certain way - any variation in birth canal shapes could be selectively disadvantageous for birth, even in taxa with 
small cephalopelvic proportions (because a birth canal shape that is highly atypical could also affect birth in these species). Additionally, it is possible that this shared pattern of integration is an ancestral character for anthropoids (or mammals). Future research should further test this hypothesis by comparing primate birth canal integration patterns to those of other mammalian taxa. 


$\begin{array}{ll}\text { Hypothesis } & \text { Results }\end{array}$

\section{Prediction}

H1: The birth canal and non-obstetric pelvis represent two separate modules

1a: In species with large CP, there will be low covariance between the birth canal and non-obstetric pelvis, but this will not be the case in taxa with small CP the birth canal

H2: Large cephalopelvic proportions produce high magnitudes of integration in

2a: Primates with large $\mathrm{CP}$ will exhibit a greater magnitude of integration in the birth canal than species with small $\mathrm{CP}$

$2 \boldsymbol{b}$ : In species with large $\mathrm{CP}$, females will exhibit a greater magnitude of integration in the birth canal than males, but this will not be the case in taxa with small CP

H3: Large cephalopelvic proportions produce high magnitudes of integration in the non-obstetric pelvis

3a: Primates with large CP will exhibit a greater magnitude of integration in the non-obstetric pelvis than species with small CP

3b: In species with large $\mathrm{CP}$, females will exhibit a greater magnitude of integration in the non-obstetric pelvis than males, but this will not be the case in taxa with small CP

H4: Large cephalopelvic proportions do not produce specific patterns of integration in the birth canal

4a: All species share similar patterns of integration in the birth canal regardless of cephalopelvic proportions

$4 \boldsymbol{b}$ : Within species, males and females will have similar patterns of integration within the birth canal

H5: Large cephalopelvic proportions do not produce specific patterns of integration in the non-obstetric pelvis

5a: All species share similar patterns of integration in the non-obstetric pelvis Not Supported regardless of cephalopelvic proportions

5b: Within species, males and females will have similar patterns of integration Supported within the non-obstetric pelvis

Cannot Reject

Not Supported

[Table 5.5]

Supported

[Table 5.7]

\section{Cannot Reject}

Supported

[Table 5.8]

Supported

[Table 5.10]

\section{Cannot Reject}

Supported

[Table 5.11]

Supported

[Table 5.12]

Cannot Reject

[Table 5.13]

*Cephalopelvic Proportions (CP)
[Table 5.14] 


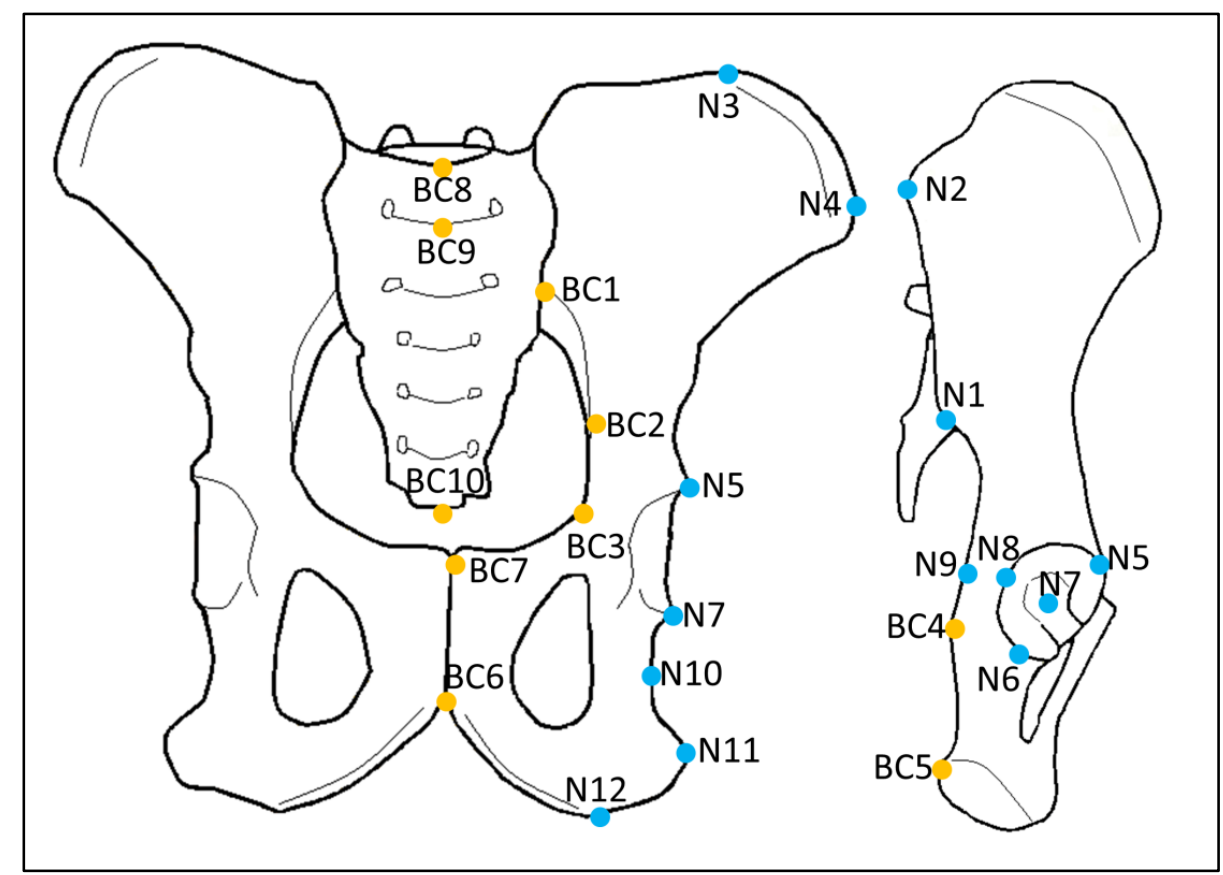

Figure 5.1: Landmarks of the birth canal (orange) and non-obstetric pelvis (blue).

Landmark descriptions are listed in Table 5.1.

\begin{tabular}{ll}
\hline Table 5.2: Landmarks of the Birth Canal \\
\hline Birth Canal Landmarks & Description \\
\hline (BC1) SI joint at arcuate line & Point on ilium where arcuate line intersects the sacroiliac joint \\
(BC2) Lateral arcuate line & Lateral-most point of arcuate on articulated pelvis \\
(BC3) Pubis at arcuate line & Arcuate at right angle to anterior acetabular rim \\
(BC4) Ischial spine & Ischial spine on posterior ischium \\
(BC5). Posterior ischial tuberosity & Posterior-most point on the ischial tuberosity \\
(BC6) Inferior pubic symphysis & Inferior-most point on symphysis at the midline \\
(BC7) Superior pubic symphysis & Superior-most point on symphysis at the midline \\
(BC8) Ventral S1body & Ventral-most point on the first vertebral body at midline \\
(BC9) Inferior S1 body & Inferior S1 vertebral body at midline \\
(BC10) Inferior sacrum & Inferior-most point on sacrum at midline \\
\hline
\end{tabular}

Table 5.3: Landmarks of the non-obstetric pelvis

\section{Non-Obstetric Landmarks}

(N1) Posterior inferior iliac spine

(N2) Posterior superior iliac spine

(N3) Superior iliac crest

(N4) Anterior superior iliac spine

(N5) Superior acetabulum

(N6) Inferior acetabulum

(N7) Acetabulum center

(N8) Posterior acetabulum

(N9) Posterior ischial neck

(N10) Lateral ischial ramus

(N11) Lateral ischial tuberosity

(N12) Inferior pubic ramus

\section{Description}

Posterior-most point of the posterior inferior iliac spine

Posterior-most point of the posterior superior iliac spine

Superior-most point on the iliac crest

Lateral-most point on the anterior superior iliac spine

Point on superior rim of acetabulum in the same plane as anterior inferior iliac spine

Point on inferior rim of acetabulum in the same plane as ischial ramus

Point in the acetabulum at the center of the lunate surface

Rim of the acetabulum in the same plane as landmarks 7 and 9

Point on the posterior ischium in the same plane as landmarks 7 and 9

Deepest point along the ischial ramus inferior to the acetabulum

Lateral-most projection of the ischial tuberosity

Point between ischial and inferior pubic rami in at lateral edge of obturator foramen 


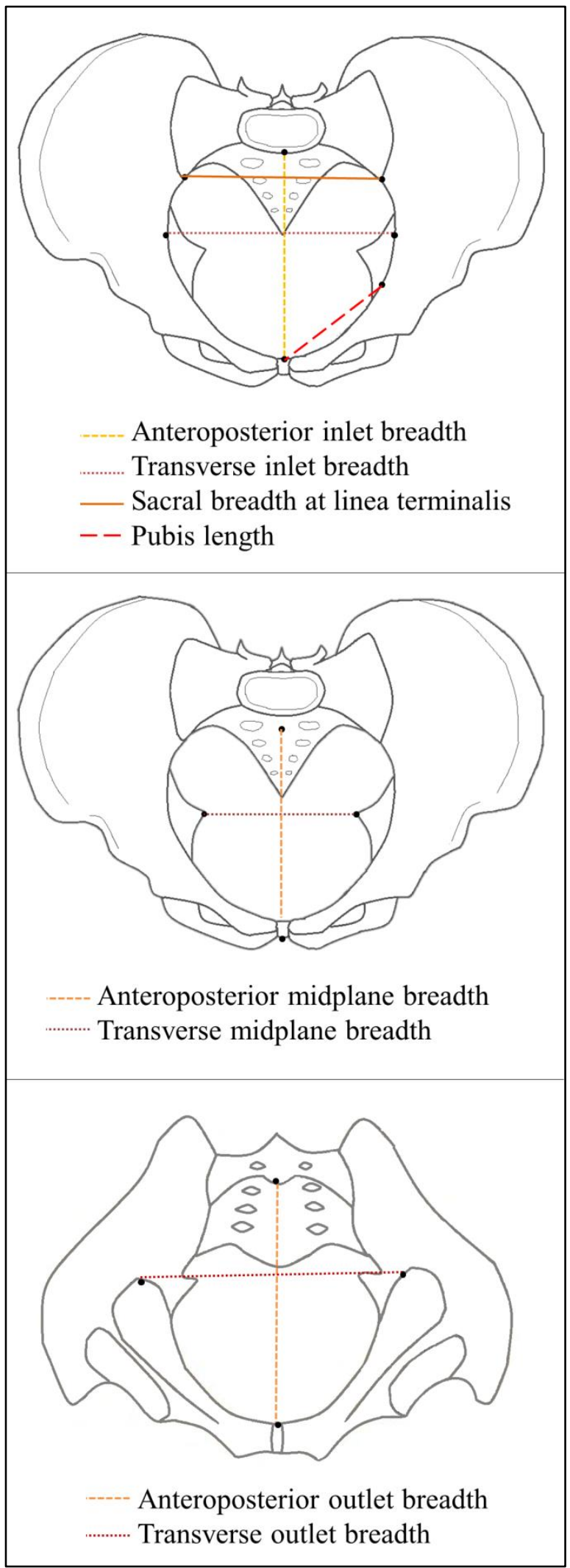

Figure 5.2: Linear distances of the birth canal. 


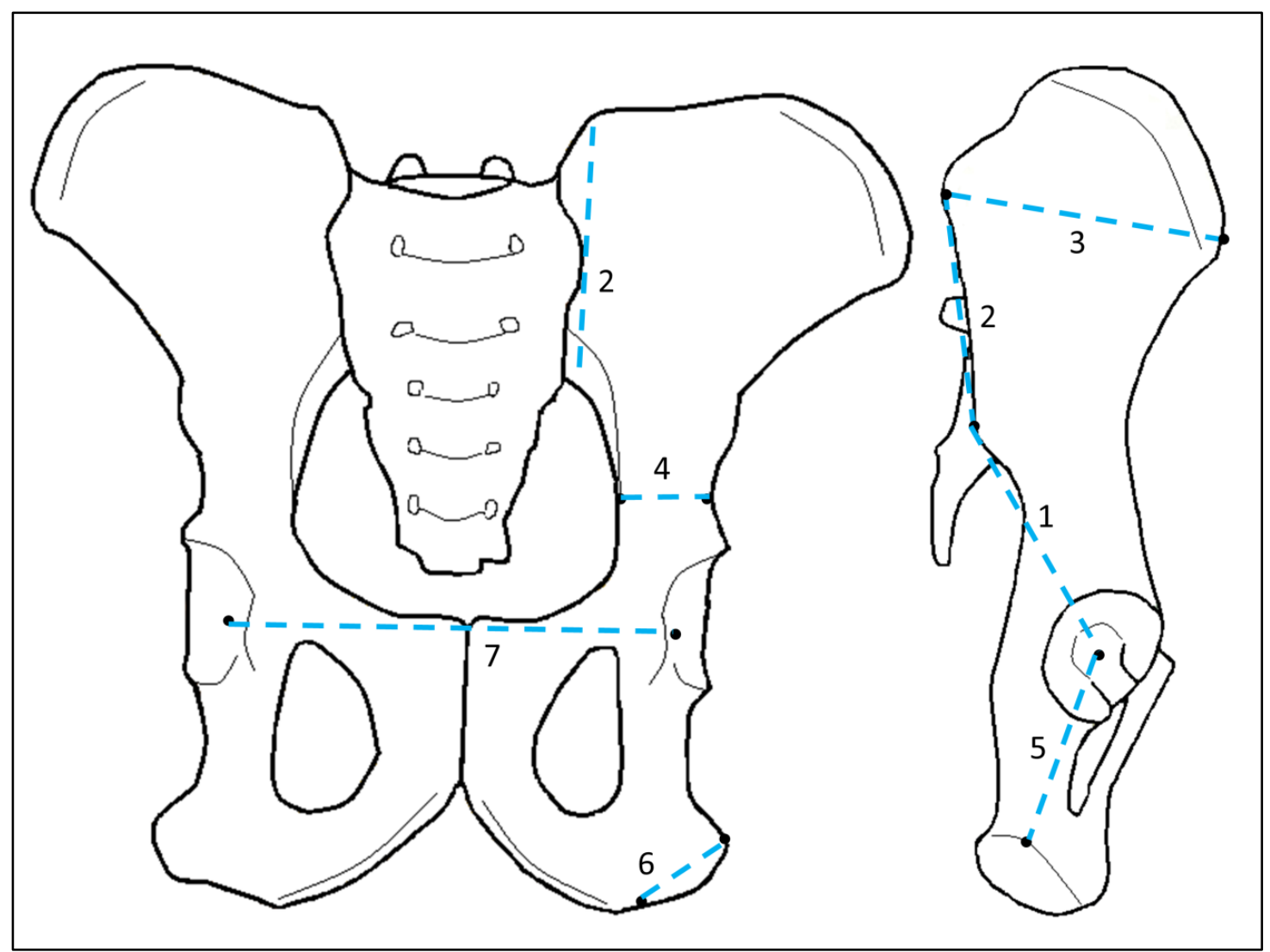

Figure 5.3: Linear distances of the non-obstetric pelvis: (1) inferior iliac height; (2) superior iliac height; (3) iliac breadth; (4) iliac neck breadth; (5) ischial length; (6) ischial tuberosity breadth; (7) biacetabular breadth. 
Table 5.4: Hypothesis 1 - Modularity testing for birth canal and non-obstetric pelvis

\begin{tabular}{lcc}
\hline Large Cephalopelvic Proportions & $\begin{array}{c}\text { Modularity Testing } \\
\mathbf{R}_{\mathbf{V}} \text { coefficient }\end{array}$ & $\begin{array}{c}\text { Proportion of } \mathbf{R}_{\mathbf{V}} \text { coefficients }< \\
\mathbf{R}_{\mathbf{V}} \text { coefficient of hypothesized modules§ }\end{array}$ \\
\hline Homo sapiens & 0.2919 & $0.0005^{*}$ \\
Hylobates lar & 0.4798 & 0.2909 \\
Macaca mulatta & 0.3417 & $0.0096^{*}$ \\
Saimiri sciureus & 0.5519 & 0.8594 \\
\hline Small Cephalopelvic Proportions & & 0.4364 \\
\hline Gorilla gorilla & 0.1893 & 0.2765 \\
Pan troglodytes & 0.4001 &
\end{tabular}

§Indicates the proportion of $\mathrm{R}_{\mathrm{V}}$ coefficients (of 10,000) for randomly-generated modules are less than the $\mathrm{R}_{\mathrm{V}}$ coefficient for the hypothesized modules (birth canal and non-obstetric pelvis).

*A significant result indicates that $\angle 5 \%$ of randomly-generated modules have lower $\mathrm{R}_{\mathrm{V}}$ coefficients than the hypothesized modules, thus a low proportion supports the hypothesis that there is support for the two hypothesized modules (i.e., birth canal and non-obstetric pelvis). 
Table 5.5: Hypothesis 2 - Magnitudes of integration in birth canal linear distances across species of anthropoids Species-Wide

Large Cephalopelvic Proportions Standardized VE $\uparrow$

Homo sapiens

0.0787

Hylobates lar

0.0932

Macaca mulatta

0.0625

Saimiri sciureus

0.0910

Small Cephalopelvic Proportions

Gorilla gorilla

0.0838

Pan troglodytes

0.0609

$\dagger$ the Variance of the Eigenvalues (VE; the measure of integration between linear distances of the birth canal used for this study) standardized by the maximum eigenvalue variance for each taxon. Standardized VE can be directly compared between taxa.

Table 5.6: Hypothesis 2 - Difference of means tests for difference in integration magnitudes (Standardized $V E)$ between taxa for birth canal distances

\begin{tabular}{ccccccl}
\hline Homo & Hylobates & Macaca & Saimiri & Gorilla & Pan & \\
\hline- & $<0.001^{*}$ & $<0.001^{*}$ & $<0.001^{*}$ & $0.023^{*}$ & $<0.001^{*}$ & Homo \\
& - & $<0.001^{*}$ & 0.056 & $0.019^{*}$ & $<0.001^{*}$ & Hylobates \\
& - & $<0.001^{*}$ & $<0.001^{*}$ & 0.521 & Macaca \\
& & - & $0.010^{*}$ & $<0.001^{*}$ & Saimiri \\
& & & - & $<0.001^{*}$ & $\begin{array}{l}\text { Gorilla } \\
\text { Pan }\end{array}$ \\
\hline
\end{tabular}

*Indicates significant difference between species for birth canal linear distances at $\alpha=0.025$

Table 5.7: Hypothesis 2 - Differences in the magnitudes of integration in birth canal linear distances between males and females across anthropoids

\begin{tabular}{lccc}
\hline & $\begin{array}{c}\text { Female } \\
\text { Standardized } \\
\text { VE }\end{array}$ & $\begin{array}{c}\text { Male } \\
\text { Standardized } \\
\text { VE }\end{array}$ & $\begin{array}{c}\text { p-value } \\
\text { (sex with higher } \\
\text { integration) }\end{array}$ \\
\hline Homo sapiens & 0.0820 & 0.0722 & $0.002 *$ (f) \\
Hylobates lar & 0.0941 & 0.0866 & $0.024 *(\mathrm{f})$ \\
Macaca mulatta & 0.0632 & 0.0647 & 0.835 \\
Saimiri sciureus & 0.0912 & 0.0835 & $0.004 *(\mathrm{f})$ \\
\hline Small Cephalopelvic Proportions & & & \\
\hline Gorilla gorilla & 0.0818 & 0.0830 & 0.672 \\
Pan troglodytes & 0.0558 & 0.0652 & $0.002 *(\mathrm{~m})$ \\
\hline
\end{tabular}

*Indicates significant difference between sexes for integration in birth canal linear distances at $\alpha=0.025$. 
Table 5.8: Hypothesis 3 - Magnitudes of integration in non-obstetric pelvic linear distances across species of anthropoids

\begin{tabular}{lc}
\hline Large Cephalopelvic Proportions & $\begin{array}{c}\text { Species-Wide } \\
\text { Standardized VE } \dagger\end{array}$ \\
\hline Homo sapiens & 0.0664 \\
Hylobates lar & 0.0678 \\
Macaca mulatta & 0.0599 \\
Saimiri sciureus & 0.0759 \\
\hline Small Cephalopelvic Proportions & \\
\hline Gorilla gorilla & 0.0582 \\
Pan troglodytes & 0.0595 \\
\hline
\end{tabular}

$\dagger$ the Variance of the Eigenvalues (the measure of integration used for this study) standardized by the maximum eigenvalue variance for each taxon. Standardized VE can be directly compared between taxa.

\begin{tabular}{|c|c|c|c|c|c|c|}
\hline \multicolumn{7}{|c|}{$\begin{array}{l}\text { Table 5.9: Hypothesis } 3 \text { - Similarity in integration magnitudes between taxa for non-obstetric pelvic } \\
\text { distances }\end{array}$} \\
\hline Homo & Hylobates & Macaca & Saimiri & Gorilla & Pan & \\
\hline \multirow[t]{6}{*}{ 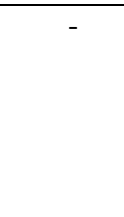 } & 0.476 & 0.039 & $0.009 *$ & $0.004 *$ & $0.005^{*}$ & Homo \\
\hline & - & $0.002 *$ & $<0.001 *$ & $0.002 *$ & $0.007 *$ & Hylobates \\
\hline & & - & $<0.001 *$ & 0.530 & 0.878 & Macaca \\
\hline & & & - & $<0.001 *$ & $<0.001 *$ & Saimiri \\
\hline & & & & - & 0.709 & Gorilla \\
\hline & & & & & - & Pan \\
\hline
\end{tabular}

*Indicates significant difference between species for birth canal linear distances at $\alpha=0.025$

Table 5.10: Hypothesis 3 - Differences in the magnitudes of integration in non-obstetric pelvic linear listances between males and females across anthropoids

\begin{tabular}{lccc} 
Large Cephalopelvic Proportions & $\begin{array}{c}\text { Female } \\
\text { Standardized } \\
\text { VE }\end{array}$ & $\begin{array}{c}\text { Male } \\
\text { Standardized } \\
\text { VE }\end{array}$ & $\begin{array}{c}\text { p-value } \\
\text { (sex with higher integration) }\end{array}$ \\
\hline Homo sapiens & 0.0535 & 0.0750 & $<0.001^{*}(\mathrm{~m})$ \\
Hylobates lar & 0.0646 & 0.0522 & $<0.001^{*}(\mathrm{f})$ \\
Macaca mulatta & 0.0797 & 0.0625 & $0.002^{*}(\mathrm{f})$ \\
Saimiri sciureus & 0.0929 & 0.0737 & $<0.001^{*}(\mathrm{f})$ \\
\hline Small Cephalopelvic Proportions & & & 0.028 \\
\hline Gorilla gorilla & 0.0667 & 0.0597 & $<0.001^{*}(\mathrm{~m})$ \\
Pan troglodytes & 0.0582 & 0.0745 &
\end{tabular}

*Indicates significant difference between sexes for integration in non-obstetric pelvic linear distances at $\alpha=0.025$. 
Table 5.11: Hypothesis 4-Mantel tests of similarity in interspecies integration patterns between birth canal traits

\begin{tabular}{cccccll}
\hline Homo & Hylobates & Macaca & Saimiri & Gorilla & Pan & \\
\hline- & $<0.001^{*}$ & $<0.001^{*}$ & $<0.001^{*}$ & $<0.001^{*}$ & $<0.001^{*}$ & Homo \\
& - & $<0.001^{*}$ & $<0.001^{*}$ & $<0.001^{*}$ & $<0.001^{*}$ & Hylobates \\
& - & $<0.001^{*}$ & $<0.001^{*}$ & $<0.001^{*}$ & Macaca \\
& & - & $0.002^{*}$ & $<0.001^{*}$ & Saimiri \\
& & & - & $<0.001^{*}$ & Gorilla \\
& & & & - & Pan \\
\hline
\end{tabular}

*Indicates a significant result at $\alpha=0.025$. Significance indicates rejection of the null hypothesis - that there is no association between matrix pairs - and therefore indicates that two species share a similar integration pattern.

\begin{tabular}{lc}
\hline $\begin{array}{l}\text { Table 5.12: Hypothesis } 4 \text { - Similarity in birth canal integration patterns between } \\
\text { males and females }\end{array}$ \\
\hline Large Cephalopelvic Proportions & Mantel Test of Matrix Similarity \\
\hline Homo sapiens & $<0.001^{*}$ \\
Hylobates lar & $<0.001^{*}$ \\
Macaca mulatta & $<0.001^{*}$ \\
Saimiri sciureus & $<0.001^{*}$ \\
\hline Small Cephalopelvic Proportions & Mantel Test of Matrix Similarity $\ddagger$ \\
\hline Gorilla gorilla & $<0.001^{*}$ \\
Pan troglodytes & $<0.001^{*}$ \\
\hline
\end{tabular}

*Indicates a significant result at $\alpha=0.025$.

$\$$ Significance of a Mantel test indicates rejection of the null hypothesis - that there is no association between matrix pairs - and therefore indicates that two groups share a similar integration pattern. 
Table 5.13: Hypothesis 5 - Mantel tests of similarity in integration patterns between non-obstetric pelvic traits

\begin{tabular}{ccccccl}
\hline Homo & Hylobates & Macaca & Saimiri & Gorilla & Pan & \\
\hline- & 0.037 & 0.233 & $0.010^{*}$ & $0.010^{*}$ & 0.042 & Homo \\
& - & 0.052 & $<0.001^{*}$ & 0.099 & 0.100 & Hylobates \\
& - & 0.171 & 0.121 & 0.096 & Macaca \\
& & - & 0.090 & 0.117 & Saimiri \\
& & & & - & $<0.01^{*}$ & Gorilla \\
\hline
\end{tabular}

*Indicates a significant result at $\alpha=0.025$. Significance indicates rejection of the null hypothesis - that there is no association between matrix pairs - and therefore indicates that two species share a similar integration pattern.

\begin{tabular}{lc}
\hline $\begin{array}{l}\text { Table 5.14: Hypothesis } 5 \text { - Similarity in non-obstetric integration patterns between } \\
\text { males and females }\end{array}$ \\
\hline Large Cephalopelvic Proportions & Mantel Test of Matrix Similarity \\
\hline Homo sapiens & $0.008^{*}$ \\
Hylobates lar & $0.005^{*}$ \\
Macaca mulatta & 0.119 \\
Saimiri sciureus & $0.003^{*}$ \\
\hline Small Cephalopelvic Proportions & Mantel Test of Matrix Similarity \\
\hline Gorilla gorilla & $0.018^{*}$ \\
Pan troglodytes & 0.031 \\
\hline
\end{tabular}

*Indicates a significant result at $\alpha=0.025$.

$\$$ Significance of a Mantel test indicates rejection of the null hypothesis - that there is no association between matrix pairs - and therefore indicates that two groups share a similar integration pattern. 


\section{CHAPTER 6: SUMMARY AND FUTURE DIRECTIONS}

The goal of this dissertation is to ascertain the relationship between obstetric demand, measured here as cephalopelvic proportions, and pelvic dimorphism and morphological integration across anthropoid primates.

The first study (Chapter 2) examines dimorphism in birth canal morphology in anthropoids. Dimorphism is found in the size and shape of the birth canal among all species included except for Alouatta seniculus, but taxa with large cephalopelvic proportions are especially dimorphic. The results of this study indicate that the presence of dimorphism in the birth canals of primates cannot be attributed to large cephalopelvic proportions because birth canal dimorphism is pervasive across anthropoid taxa regardless of cephalopelvic proportions. In all species except $A$. seniculus, females have relatively larger birth canals than males, but female birth canals are not isometrically larger - female birth canals tend to be relatively mediolaterally wider, but not necessarily relatively larger in the anteroposterior dimensions, and females also have relatively longer pubes (all taxa except $A$. seniculus), which contributes to mediolateral widening of the birth canal planes.

An important implication is that birth canal dimorphism may be a shared feature of most primate taxa. Indeed, dimorphic pubis and birth canal morphology has also been observed in other eutherian mammals (including rodents, bats, and foxes; Gardner, 1936; Dunmire, 1955; Crelin and Newton, 1969; Leutenegger and Kelly, 1977; 
Chapman et al., 1994; Berdnikovs et al., 2007; Schutz et al., 2009a; Schutz et al., 2009b) as well as in metatherians (the opossum; Tague, 2003), which have small cephalopelvic proportions. In all of these taxa, females have relatively longer pubis length than males, and increasing pubis length necessarily increases birth canal size in females. Sex differences in pubis length could be a shared feature of a number of mammalian taxa (or a shared feature of mammals), but further research is needed to test this hypothesis. Sex differences in relative pubis length and mediolateral dimensions of the birth canal may therefore be especially useful for inferring biological sex from fossil pelves.

Sex hormones likely play a strong role in the determination of adult dimorphism patterns in mammals (Gardner, 1936; Dunmire, 1955; Tague, 1995). Previous researchers have hypothesized that pelvic dimorphism may be attributable to differences between the sexes in the presence of either testosterone (Tague, 1995) and/or estrogen (Gardner, 1936) during development. Further, dramatic sex differences in the pelvis begin to develop during early adolescence in most primates that have been studied previously ( $\mathrm{Li}, 2002)$. The results of this study suggest that these developmental pathways are likely shared across most species of primates regardless of cephalopelvic proportions, because most taxa have some level of pelvic dimorphism in adults. Future research should consider the role of sex hormones on birth canal form across primates during ontogeny.

Importantly, this study shows that the magnitude of dimorphism in birth canal size and shape is, in fact, related to cephalopelvic proportions. Species with large cephalopelvic proportions, which are presumably under increased obstetric selection 
compared to species with small proportions, tend to be more dimorphic than small proportion taxa. Furthermore, species with both high levels of body size dimorphism and large cephalopelvic proportions tend to have the most dimorphic birth canals. Previous researchers have noted that primates with high levels of body size dimorphism also have high levels of pelvic dimorphism (Schultz, 1949; Leutenegger, 1973; Tague, 1995; 2005). The reasons for this are twofold. First, secondary sex differences (including both body size dimorphism and pelvic dimorphism) in primates are thought to be influenced by sex hormone levels during ontogeny (Tague, 1995; Li, 2002; Tague, 2005), so species with high levels of body size dimorphism should also be expected have heightened levels of pelvic dimorphism (Schultz, 1949; Tague, 1995; 2005). Second, in taxa with high levels of body size dimorphism, small-bodied females are at an obstetric disadvantage (a) because they have small pelves and (b) because neonatal body size is negatively allometric with maternal body size, meaning they have relatively large neonates (Leutenegger, 1982). Therefore, obstetric selection in these small-bodied females is very high, resulting in females with very large birth canals controlling for body size compared to those of males.

The second study (Chapter 3) examines whether dimorphism in sacral breadth is due to large cephalopelvic proportions. Dimorphism in sacral breadth relative to S1 body breadth is present among all species having large cephalopelvic proportions as well as Gorilla gorilla (which has small cephalopelvic proportions), but this may be due to males exhibiting relatively narrow sacra controlling for $\mathrm{S} 1$ body breadth in G. gorilla (see Chapter 3). No other species having small cephalopelvic proportions exhibit dimorphism in sacral breadth relative to $\mathrm{S} 1$ body breadth. This result helps to explain 
why taxa with large cephalopelvic proportions also have high magnitudes of dimorphism in birth canal size and shape compared to those with small proportions. The sacrum makes up the posterior border of the pelvic inlet, midplane, and outlet, and increasing sacral breadth necessarily increases the distance between the posterior ilia and ischia and increases the mediolateral breadth (and therefore the circumference) of the birth canal in females. If sacral breadth is significantly dimorphic in species with large cephalopelvic proportions, mediolateral dimensions and circumferences of the birth canal would be expected to be highly dimorphic in these taxa as well, which is exactly what is observed in Chapter 2 .

Although the sacrum is often ignored in studies of pelvic form, the results from this dissertation indicate that fossil sacra may provide important information regarding the evolution of cephalopelvic proportions across hominins in taxa for which we have sacra for male and female individuals. There are a number of well-preserved hominin fossil sacra (Leutenegger, 1977; Johanson et al., 1982; Rak and Arensburg, 1987; Arsuaga et al., 1999; Toussaint et al., 2003; Simpson et al., 2008; Bonmatí et al., 2010; Kibii et al., 2011). Tague (2007) has measured relative sacral breadth in three fossil sacra attributed to Australopithecus: A.L. 288-1 (A. afarensis, typically considered to be female; Johanson and Edey, 1981; Tague and Lovejoy, 1986; but see Häusler and Schmid, 1995), Sts 14 (A. africanus, typically considered female; Howell, 1978; but see Robinson, 1972), and Stw 431 (A. africanus, typically considered male; Toussaint et al., 2003). Tague's indices of relative sacral breadth in these specimens indicate that the two female specimens do have relatively wider sacra than males, but it is possible that this finding is due to small sample size (Tague, 2007). However, if we assume that these 
individuals are representative of the mean sacral morphology for Australopithecus, the index of dimorphism in Australopithecus is similar to that seen in Homo sapiens, Hylobates lar, and Gorilla gorilla. There are two possible interpretations of this finding: (1) Australopithecus was characterized by large cephalopelvic proportions, or (2) high levels of body size dimorphism in Australopithecus (McHenry, 1991; Richmond and Jungers, 1995; Gordon and Green, 2008; but see Reno et al., 2003) influenced sacral dimorphism in this taxon, similar to what we find in G. gorilla. It is unlikely that Australopithecus was characterized by large cephalopelvic proportions similar to those in humans (Leutenegger, 1972; but see Berge et al., 1984), although cephalopelvic proportions would almost certainly have been larger than those in Pan or Gorilla (Berge et al., 1984; Tague and Lovejoy, 1986). Therefore, the most reasonable interpretation of high levels of dimorphism in relative sacral breadth in Australopithecus (if this is not an artifact of small sample size) is that this taxon was characterized by high levels of body size dimorphism, and similar to Gorilla gorilla, there may have been slight differences in locomotor function between males and females owing to these body size differences (Stern and Susman, 1983). Data do not exist for relative sacral breadth in other fossil hominins. Thus, future research should aim to reconstruct the evolution of cephalopelvic proportions across fossil hominins using sacral morphology.

Dimorphism in sacral breadth and birth canal morphology influences dimorphism in the non-obstetric pelvis. The third study of this thesis (Chapter 4) finds that shape and size differences in the non-obstetric pelvis are apparent in all species included with the exception of Alouatta seniculus (as would be expected based on the results for the presence of birth canal dimorphism in primates). As with the birth canal, 
the presence of dimorphism in non-obstetric pelvic size and shape is not indicative of large cephalopelvic proportions. Yet, similar to what was found in the birth canal, species with large cephalopelvic proportions are especially dimorphic for non-obstetric pelvic shape and size, such that there is a greater magnitude of dimorphism in pelvic size and less overlap in pelvic shape in these taxa. Generally, the magnitude of dimorphism in the non-obstetric pelvis is a corollary of the magnitude of dimorphism in the birth canal.

Size and shape differences in the non-obstetric pelvis are similar across most taxa, and the parts of the non-obstetric pelvis that are adjacent to the birth canal - the inferior ilium and ischiopubis - are the most dimorphic. Females have relatively wider (but not rostrocaudally larger) ischiopubic regions than males, and females have relatively wider biacetabular breadth in all species except A. seniculus and M. mulatta. Lower iliac height (distance between sacroiliac and acetabulum) is relatively larger in females than males in all species except A. seniculus. However, primates exhibit little dimorphism in the rostral ilium, which is spatially separated from the birth canal in most species. Relative biiliac breadth is not dimorphic controlling for body size in any species (although it is dimorphic for most taxa when allometric scaling is considered). Collectively, these results show that the inferior ilium (which includes the linea arcuata, lateral boundary of the pelvic inlet) and ischiopubis (which bounds the pelvic inlet, midplane, and outlet) tend to be very dimorphic in anthropoids, such that these areas are relatively wider in females than males. This is in contrast to the upper ilium, which does not become relatively wider in females due to strong positive allometric scaling of biiliac breadth (Lewton, 2015). The fact that biiliac breadth is not dramatically wider in 
females does not pose an obstetric disadvantage, because this area is spatially separated from the birth canal.

Chapters 2-4 of this thesis establish that species with large cephalopelvic proportions are highly dimorphic for birth canal, sacral, and non-obstetric pelvic morphology, and it is clear that obstetric selection plays a role in constraining female pelvic form in these species. This does not appear to be the case in species with small cephalopelvic proportions, which tend to have less dimorphism in their birth canals, sacra, and non-obstetric pelves. Therefore, the final study of this thesis (Chapter 5) tests the hypothesis that large cephalopelvic proportions increase the magnitude of integration (covariation) in the birth canal and non-obstetric pelvis. In species with large cephalopelvic proportions, females have higher magnitudes of covariation (more integration) between linear distances of the birth canal (all species except M. mulatta) and between linear distances of the non-obstetric pelvis (all species except $H$. sapiens) compared to males. This is not the case in species with small cephalopelvic proportions. This result indicates that there is strong selection on females in species with large cephalopelvic proportions to maintain an obstetrically-sufficient pelvis, and this strong selection maintains high levels of integration between pelvic morphologies in these females. This is not the case in females in taxa with small proportions. This is the first study to examine and establish a difference in the magnitudes of integration in male and female anthropoid pelves. This study shows that there is a demonstrable effect of obstetric demand on pelvic integration in females in taxa with large cephalopelvic proportions. 
There are two species included in this thesis that are unusual compared to other taxa - the first is Alouatta seniculus, which is nearly monomorphic in pelvic morphology (not found in any other taxa included), and this consistent with previous research (Leutnegger, 1970). Alouatta it is the only known monkey which exhibits small cephalopelvic proportions (hypothesized to be due to decreased cranial capacity in this species; Leutenegger, 1970). It is possible that this decrease in cephalopelvic proportions in Alouatta compared to other monkeys dramatically reduced obstetric selection in this taxon. Reduced obstetric selection allows female pelves to respond to other selective regimes (for example, locomotion or thermoregulation), producing more "male-like" pelves in females. Alternatively, it is possible that pelvic monomorphism in Alouatta is due to growth rates in males. It is hypothesized that male Alouatta experience a longer skeletal growth period and faster growth rate during ontogeny than females (Ravosa and Ross, 1994), and this longer, faster growth rate in males could also lead to reduced pelvic dimorphism by increasing pelvic size and breadth in male pelves to be similar to that of females.

The second species which is unusual is Homo sapiens. Humans have more dimorphic pelves than would be predicted based on our cephalopelvic proportions or body size dimorphism levels alone (see Chapters 2 and 4), and, notably, humans are the only bipedal primates included. In humans, the birth canal and non-obstetric pelvis are hypothesized to be under antagonistic pressures of selection for obstetric sufficiency and selection for bipedal locomotion (termed the "obstetric dilemma"; Washburn, 1960; Pinkerton, 1973; Zihlman, 1978; Ruff, 1995; Lovejoy, 2005). A larger, wider birth canal is beneficial for birth, but a wider biacetabular distance is thought to be 
energetically costly in bipeds because it increases the abductor forces required to prevent hip drop (Washburn, 1950, 1960; Pinkerton, 1973; Zihlman, 1978; Ruff, 1995; Lovejoy, 2005; but see Warrener et al., 2015). A wider birth canal also increases the distance between the ischial spines, possibly compromising the function of the pelvic diaphragm in pelvic visceral support in bipeds (Abitbol, 1988), and it increases valgus angle, which could lead to increased incidence of knee injuries (see Warrener et al., 2015). These antagonistic selection pressures between obstetric and locomotor functions are also reflected in the morphological integration in the pelvis - humans have more modular pelves (less covariation between the birth canal and non-obstetric pelvis) compared to most other primates (Chapter 5; see also Grabowski et al., 2011). This allows for differential selection on the morphology of the birth canals and the nonobstetric pelves in humans - while the birth canal is more tightly integrated in human females (who give birth), the non-obstetric pelvis displays higher magnitudes of integration in human males (who are only under selection for bipedal locomotion and thermoregulatory imperatives). No other species shows this pattern of having females with higher integration in the birth canal and males with higher integration in the nonobstetric pelvis. Based on the results of this study it appears reasonable to suggest that selection for obstetric demand and selection for bipedal locomotion are at odds in our species (Washburn, 1960; Rosenberg, 1992; Ruff, 1995; Rosenberg and Trevathan, 1996), resulting in both (a) a high magnitude of dimorphism in human pelves and (b) a lesser degree of covariation between the birth canal and non-obstetric pelvis.

The results of this study have implications for sexing fossil anthropoid pelves. In previous studies, patterns of dimorphism in modern humans have been used as the basis 
to determine sex in fossil hominins (Tague and Lovejoy, 1986; Hager, 1989; Häusler and Schmid, 1995; Tague and Lovejoy, 1998; Simpson et al., 2008; Bonmatí et al., 2010). Hager (1989) noted one obvious problem with doing this. Modern humans have extremely dimorphic pelves (Turner, 1885; Caldwell and Moloy, 1933; Greulich, 1938; Young and Ince, 1940; Coleman, 1969; Krogman and Işcan, 1986; Hager, 1989; Tague, 1989, 1992; Walrath, 1997; Correia et al., 2005; Kurki, 2007; Brown, 2011; Kurki, 2011), and using modern human dimorphism patterns to sex fossils presumes that extinct hominins had similar patterns and magnitudes of pelvic dimorphism as modern humans. Ideally, any pelvic characters that are used to assign sex to fossils should be good indicators of sex in most anthropoid taxa, or at least most hominoids, not just in humans. This thesis establishes a number of pelvic traits which are dimorphic in nearly all anthropoids included (controlling for body size). These include pubis length, mediolateral diameter of the pelvic inlet, biacetabular breadth, and lower iliac height. These linear distances tend to be relatively longer in females than males in anthropoid species regardless of cephalopelvic proportions, so these distances are appropriate for use as sex discriminators in extinct species, even those with small cephalopelvic proportions. Notably, pubis length and inferior iliac height can be measured on pelves that do not preserve a complete birth canal, and lower iliac height (distance between sacroiliac and hip joint) is often preserved in fragmentary fossil hominin pelves (see Rose, 1984; Brown et al., 1985; Simpson et al., 2008; Kibii et al., 2011; Ward et al., 2015).

In humans, difficult and prolonged birth (due to large cephalopelvic proportions and neonatal rotation) is a hallmark of our species, while other great apes have small 
cephalopelvic proportions and relatively quick births (Schultz, 1949; Washburn, 1960; Lindberg, 1982; Rosenberg, 1996). In addition, the appearance of large cephalopelvic proportions and a prolonged birth process in hominins is linked to increased birth and postpartum assistance to mothers (which requires increased cooperation between individuals; Trevathan, 1996; Rosenberg and Trevathan, 2002), increased infant dependency (due to neonates being born with less brain development complete compared to other apes; Martin, 1983; DeSilva and Lesnik, 2008; DeSilva, 2011), and increased alloparenting in hominins compared to non-human primates (Hrdy, 2007; 2009). It has therefore been a critical point of research in the past several decades to reconstruct and ascertain cephalopelvic proportions and birth-related behavior in fossil hominins (Tague and Lovejoy, 1986; Rosenberg, 1992; Häusler and Schmid, 1995; Ruff, 1995; Rosenberg and Trevathan, 1996; Trevathan, 1996; Rosenberg and Trevathan, 2002; Coqueugniot et al., 2004; DeSilva and Lesnik, 2008; Ponce de León et al., 2008; Franciscus, 2009; Weaver and Hublin, 2009; DeSilva, 2011). Cephalopelvic proportions and birth-related selection are often inferred in hominins from dimorphism in pelvic morphology (Zihlman, 1978; Berge et al., 1984; Tague and Lovejoy, 1986; Rak and Arensburg, 1987; Hager, 1989; Arsuaga et al., 1999; Simpson et al., 2008; Bonmatí et al., 2010; Ruff, 2010), but results from this dissertation indicate that the presence of dimorphism in the birth canal and non-obstetric pelvic morphology (see Chapters 2 and 3) is insufficient to indicate that a species has large cephalopelvic proportions. Indeed, most species of anthropoids included in these studies exhibit some dimorphism in pelvic morphology regardless of cephalopelvic proportions. Therefore, 
any indicators of large cephalopelvic proportions in fossils should also be shared by (and be exclusive to) extant taxa with large cephalopelvic proportions.

Based on the results of this thesis, it would be expected that any anthropoid species with large cephalopelvic proportions would be characterized by: (1) dimorphism in relative sacral breadth; (2) high magnitudes of dimorphism in the size and shape of the birth canal; and (3) high magnitudes of dimorphism in biacetabular breadth and lower iliac height. Moreover, in species with large cephalopelvic proportions females should have greater magnitudes of birth canal integration than males (see Chapter 5), although this would be nearly impossible to detect in fossils due to small sample sizes.

Finally, there were two limitations of this thesis that should be addressed with future research. First, there is limited data on cephalopelvic proportions across anthropoids. Future research should quantify cephalopelvic proportions in a larger number of taxa using ultrasound or radiography. Second, this study quantified obstetric demand as bony constraints between the neonatal head and maternal pelvis, but it is possible that there are other factors - including wide neonatal shoulders (Trevathan and Rosenberg, 2000) and soft tissue constraints (including pelvic floor musculature, pelvic ligament deformation, or neonatal cranial deformation due to fontanelles) - which influence the magnitude of obstetric demand in many taxa. Addressing these limitations will create a more complete picture of how obstetric demand correlates with pelvic form in female primates. 


\section{WORKS CITED:}

Abitbol MM. 1987a. Evolution of the sacrum in hominoids. American Journal of Physical Anthropology 74:65-81.

Abitbol MM. 1987b. Obstetrics and posture in pelvic anatomy. Journal of Human Evolution 16:243-255.

Abitbol MM. 1988. Evolution of the ischial spine and of the pelvic floor in the hominoidea. American Journal of Physical Anthropology 75:53-67.

Abitbol MM. 1991. The cephalopelvic disproportion index. Combined fetal sonography and x-ray pelvimetry for early detection of cephalopelvic disproportion. American Journal of Physical Anthropology 85:135-148.

Abitbol MM. 1996. The shapes of the female pelvis: contributing factors. Journal of Reproductive Medicine 41:242-250.

Acheson RM, Jefferson E. 1954. Some observations on the closure of the anterior fontanelle. Archives of Diseases in Childhood 29:196-198.

Acker DS, Sachs BP, Friedman EA. 1985. Risk factors for shoulder dystocia. Obstetrics and Gynecology 66:762-768.

Ackermann R, Cheverud J. 2000. Phenotypic covariance structure in tamarins (genus Saguinus): a comparison of variation patterns using matrix correlation and common principal components analysis. American Journal of Physical Anthropology 111:489-501. 
Adair F. 1918. The ossifications centers of the fetal pelvis. Transactions of the American Gynecological Society 43:89-104.

Agrawal AA. 2001. Phenotypic plasticity in the interactions and evolution of species. Science 294:321-326.

Aisenson MR. 1950. Closing of the anterior fontanelle. Pediatrics 6:223-226.

Arsuaga J-L, Carretero JM. 1994. Multivariate analysis of the sexual dimorphism of the hip bone in a modern human population and in early hominids. American Journal of Physical Anthropology 93:241-257.

Arsuaga J-L, Lorenzo C, Carretero JM, Gracia A, Martínez I, García N, Bermúdez de Castro J-M, Carbonell E. 1999. A complete human pelvis from the Middle Pleistocene of Spain. Nature 399:255-258.

Atchley WR, Gaskins C, Anderson D. 1976. Statistical properties of ratios. I. empirical results. Systematic Zoology 25:137-148.

Badoux DM. 1974. An introduction to biomechanical principles in primate locomotion and structure. In: Jenkins FA, editor. Primate locomotion. New York: Academic Press.

Benazzi S, Maestri C, Parisini S, Vecchi F, Gruppioni G. 2009. Sex assessment from the sacral base by means of image processing. Journal of Forensic Science $54: 249-254$. 
Benedetti TJ, Gabbe SG. 1978. Shoulder dystocia: A complication of fetal macrosomia and prolonged second stage of labor with midpelvic delivery. Obstetric Gynecology 52:526-529.

Benjamini Y, Hochberg Y. 1995. Controlling the false discovery rate: A practical and powerful approach to multiple testing. Journal of the Royal Statistical Society Series B Statical Methodology 57:289-300.

Benton R. 1967. Morphological evidence for adaptations within the epaxial region of the primates. In: Vagtborg H, editor. The Baboon in Medical Research. Austin: University of Texas Press.

Berdnikovs S, Bernstein M, Metzler A, German RZ. 2007. Pelvic growth: ontogeny of size and shape sexual dimorphism in rat pelves. Journal of Morphology 268:1222.

Berge C, Gommery D. 1999. Le sacrum de Sterkfontein Sts 14Q (Australopithecus africanus): Nouvelles données sur la croissance et sur l'âge osseux du spécimen (homage à R. Broom et J.T. Robinson). C R Acad Sci Série IIA Sciences de la terre et des planètes 329:227-232.

Berge C, Orban-Segebarth R, Schmid P. 1984. Obstetrical interpretation of the australopithecine pelvic cavity. Journal of Human Evolution 13:573-587.

Biewener AA. 1983. Bone stress in the horse forelimb during locomotion at different gaits: a comparison of two experimental methods. Journal of Biomechanics $16: 565-576$. 
Biewener AA. 1990. Biomechanics of mammalian terrestrial locomotion. Science (New York, NY) 4984:1097-1103.

Biewener AA. 2005. Biomechanical consequences of scaling. The Journal of Experimental Biology 208:1665-1676.

Blomberg SP, Garland T, Ives AR. 2003. Testing for phylogenetic signal in comparative data: behavioral traits are more labile. Evolution 57:717-745.

Bollow M, Braun J, Kannenberg J, Biederman T, Schauer-Petrowskaja C, Paris S, Mutze S, Hamm B. 1997. Normal morphology of the sacroiliac joints in children: magnetic resonance studies related to age and sex. Skeletal Radiology 26:697-704.

Bonmatí A, Gómez-Olivencia A, Arsuaga J-L, Carretero JM, Gracia A, Martínez I, Lorenzo C, Bérmudez de Castro JM, Carbonell E. 2010. Middle Pleistocene lower back and pelvis from an aged human individual from the Sima de los Huesos site, Spain. Proceedings of the National Academy of Science USA 107:18386-18391.

Bonner J. 1988. The Evolution of Complexity. Princeton, New Jersey: Princeton University Press.

Bookstein F. 1991. Morphometric tools for landmark data: Geometry and biology. Cambridge: Cambridge University Press. 
Bookstein F, Gunz P, Mitteroecker P, Prossinger H, Schaefer K, Seidler H. 2003. Cranial integration in Homo: singular warps analysis of the midsagittal plane in ontogeny and evolution. Journal of Human Evolution 44:167-187.

Bookstein F, Sampson PD, Streissguth AP, Barr HM. 1990. Measuring "dose" and "response" with multivariate data using partial least squares techniques. Communication in Statistics [Section A]-Theory and Methods 19:765-804.

Boubli JP, Rylands AB, de la Torre S, Stevenson P. 2008. Saimiri sciureus: International Union for Conservation of Nature $\mathrm{N}$ red list of threatened species. Version 2011.2.

Bowden D, Winter P, Ploog D. 1967. Pregnancy and delivery behavior in the squirrel monkey (Saimiri sciureus) and other primates. Folia Primatologica 5:1-42.

Buettner-Janusch J, Mason GA, Dame L, Buettner-Janusch V, Sade DS. 1974. Genetic studies of serum transferrins of free-ranging rhesus macaues of Cayo Santiago, Macaca mulatta. American Journal of Physical Anthropology 41:217-231.

Buettner-Janusch J, Sockol M. 1977. Genetic studies of free-ranging macaques of Cayo Santiago II. 6-Phosphogluconate dehydrogenase and NADH-methemoglobin reductase (NADH-diaphorase). American Journal of Physical Anthropology 47:375-379.

Brandon-Jones D, Eudey AA, Groves GT, Melnick DJ, Morales JC, Shekelle M. 2004. Asian primate classification. International Journal of Primatology 25:97-164. 
Brandt EM, Mitchell G. 1971. Parturition in primates: behavior related to birth. In: Rosenblum LA, editor. Primate Behavior: Developments in Field and Laboratory Research. New York: Academic Press. p 177-223.

Brown KM. 2011. Obstetrical adaptation in the human bony pelvis: a three-dimensional morphometric approach. In. Baltimore, Maryland: Johns Hopkins University.

Brown F, Harris J, Leakey R, Walker A. 1985. Early Homo erectus skeleton from west Lake Turkana, Kenya. Nature 316:788-792.

Caldwell WE, Moloy HC. 1933. Anatomical variations in the female pelvis and their effect in labor with a suggested classification. American Journal of Obstetric Gynecology 26:479-505.

Caldwell WE, Moloy HC. 1938. Anatomical variations in the female pelvis: their classification and obstetrical significance. Proceedings of the Royal Society of Medicine 32:1-30.

Caldwell WE, Moloy HC, D'esposo D. 1934. A roentgenologic study of the mechanism of engagement of the fetal head. American Journal of Obstetric Gynecology 26:824-841.

Calkins LA. 1939. The etiology of occiput presentations. American Journal of Obstetric Gynecology 38:618-623.

Chapman A, Hall LS, Bennett MB. 1994. Sexual dimorphism in the pelvic girdle of Australian flying foxes. Australian Journal of Zoology 42:261-265. 
Cheverud J. 1988. A comparison of genetic and phenotypic correlations. Evolution 42:958-968.

Cheverud J. 1996. Developmental integration and the evolution of pleiotropy. American Zoologist 36:44-50.

Christen A. 1974. Fortpflanzungsbiologie und Verhalten bei Cebuella pygmaea und Tamarin tamarin (Primates, Platyrrhina, Callithricidae); mit 22 Tab. Berlin; Hamburg: Parey.

Cole TM. 2002. WinEDMA 1.0.1 beta (2/1/2002): Windows-Based Software for Euclidean Distance Matrix Analysis.

Cole TM, Lele S. 2002. Bootstrap-based methods for comparing morphological integration patterns. American Journal of Physical Anthropology 34:55.

Coleman WH. 1969. Sex differences in the growth of the human bony pelvis. American Journal of Physical Anthropology 31:125-151.

Coqueugniot H, Hublin JJ, Veillon F, Houet F, Jacob T. 2004. Early brain growth in Homo erectus and implications for cognitive ability. Nature 431:299-302.

Correia H, Balseiro M, De Areia M. 2005. Sexual dimorphism in the human pelvis: testing a new hypothesis. Journal of Comparative Human Biology 56:153-160.

Crelin ES, Newton EV. 1969. The pelvis of the free-tailed bat: sexual dimorphism and pregnancy changes. Anatomical Record 164:349-357. 
Dalstra M, Huiskes R. 1995. Load transfer across the pelvic bone. Journal of Biomechanics 28:715-724.

Darwin C. 1896. The Descent of Man and Selection in Relation to Sex. New York: D. Appleton and Company.

Davies DP, Ansari BM, Cooke TJ. 1975. Anterior fontanelle size in the neonate. Archives of Diseases in Childhood 50:81-83.

De Souza DH. 1913. The measurements of the pelvis, with special reference to obstetric prediction. Biometrika 9:486-529.

Derry DE. 1912. The influence of sex on the position and composition of the human sacrum. Journal of Anatomy and Physiology 46:184-192.

DeSilva JM. 2011. A shift toward birthing relatively large infants early in human evolution. Proceedings of the National Academy of Science USA 108:10221027.

DeSilva JM, Lesnik JJ. 2008. Brain size at birth throughout human evolution: a new method for estimating neonatal brain size in hominins. Journal of Human Evolution 55:1064-1074.

Dixit SG, Kakar S, Agarwal S, Choudhry R. 2007. Sexing of human hip bones of Indian origin by discriminant function analysis. Journal of Forensic and Legal Medicine 14:429-435. 
Duc G, Largo RH. 1986. Anterior fontanelle: size and closure in term and preterm infants. Pediatrics 78:904-908.

Duggleby CR, Haseley PA, Rawlins RG. 1986. An overview of blood group genetic studies on the Cayo Santiago rhesus monkeys. In: The Cayo Santiago Macaques: History, Behavior, and Biology (Rawlins RG and Kessler MJ, eds.). State of University of New York Press.

Dunmire WW. 1955. Sex dimorphism in the pelvis of rodents. Journal of Mammalogy $36: 356-361$.

Dunsworth HM, Warrener AG, Deacon T, Ellison PT, Pontzer H. 2012. Metabolic hypothesis for human altriciality. Proceedings of the National Academy of Science USA 109:15212-15216.

Edgington ES. 1995. Randomization Tests. New York: Marcel Deckker, Inc.

Emmons AB. 1913. A study of the variations in the female pelvis, based on observations made on 217 specimens of the American Indian squaw. Biometrika 9:34-57.

Fairbairn DJ, Blanckenhorn WU. 2007. Sex, size, and gender roles : evolutionary studies of sexual size dimorphism. Oxford: Oxford University Press.

Faix RG. 1982. Fontanelle size in black and white term newborn infants. Journal of Pediatrics 100:304-306. 
Falk D, Zollikofer CPE, Morimoto N, Ponce de Leon MS. 2012. Metopic suture of Taung (Australopithecus africanus) and its implications for hominin brain evolution. Proceedings of the National Academy of Science USA 109:84678470.

Faro C, Benoit B, Wegrzyn P, Chaoui R, Nicolaides KH. 2005. Three-dimensional sonographic description of the fetal frontal bones and metopic suture. Ultrasound in Obstetrics and Gynecology 26:618-621.

Fawcett E. 1938. The sexing of the human sacrum. Journal of Anatomy 72:633.

Flander LB. 1978. Univariate and multivariate methods for sexing the sacrum. American Journal of Physical Anthropology 49:103-110.

Flander LB, Corruccini RS. 1980. Shape differences in the sacral alae. American Journal of Physical Anthropology 52:399-403.

Fleagle JG. 1978. Locomotion posture and habitat utilization in two sympatric Malaysian leaf monkeys (Presbytis obscura and P. melalophos). In: The Ecology of Arboreal Folivores. Ed: Montgomery GG. Washington: Smithsonian Institutue.

Fleagle JG. 1985. Size and Adaptation in Primates. In: Jungers WL, editor. Size and Scaling in Primate Biology. New York: Springer. 
Franciscus RG. 2009. When did the modern human pattern of childbirth arise? New insights from an old Neandertal pelvis. Proceedings of the National Academy of Science USA 106:9125-9126.

Fuchs AR, Fuchs F. 1984. Endocrinology of human parturition: a review. British Journal of Obstetrics and Gynecology 91: 948-967.

Gardner WU. 1936. Sexual dimorphism of the pelvis of the mouse, the effect of estrogenic hormones upon the pelvis and upon the development of scrotal hernias. Developmental Dynamics 59:459-483.

Germain G, Ferre F. 1987. Hormones et parturition chez les primates. Annales d'Endocrinologie (Paris) 48:311-321.

Goldsmith LT, Weiss G, Steinetz B. 1995. Relaxin and its role in pregnancy. Endocrine Disorders in Pregnancy 24:171-186.

Good P. 1994. Permutation Tests: A Practical Guide to Resampling Methods for Testing Hypotheses. New York: Springer-Verlag.

Gordon AD. 2006. Scaling of size and dimorphism in primates II: Macroevolution. International Journal of Primatology 27:63-105.

Gordon AD, Green DJ. 2008. Strong postcranial size dimorphism in Australopithecus afarensis: results from two new resampling methods for multivariate data sets with missing data. American Journal of Physical Anthropology 135: 311-328. 
Grabowski MW. 2013. Hominin obstetrics and the evolution of constraints.

Evolutionary Biology 40:57-75.

Grabowski MW, Polk JD, Roseman CC. 2011. Divergent patterns of integration and reduced constraint in the human hip and the origins of bipedalism. Evolution $65: 1336-1356$.

Greulich WW. 1938. The dimensions of the pelvic inlet of 789 white females. Anatomical Record 72:45-51.

Gross TL, Sokol RJ, Williams T, Thompsona K. 1987. Shoulder dystocia: A fetalphysician risk. American Journal of Obstetric Gynecology 156:1408-1418.

Hager LD. 1989. The evolution of sex differences in the hominid bony pelvis. In. Berkeley, California: University of California at Berkeley.

Hager LD. 1996. Sex differences in the sciatic notch of great apes and modern humans. American Journal of Physical Anthropology 99:287-300.

Hallgrímsson B, Willmore K, Hall BK. 2002. Canalization, developmental stability, and morphological integration in primate limbs. American Journal of Physical Anthropology 119:131-158.

Hansen TF, Bartoszek K. 2012. Interpreting the evolutionary regression: the interplay between observational and biological errors in phylogenetic comparative studies. Systematic Biology 61:413-425. 
Hansen TF, Houle D. 2008. Measuring and comparing evolvability and constraint in multivariate characters. Journal of Evolutionary Biology 2:1201-1219.

Harlow LL. 2005. Matrices and multivariate methods. In: The essence of multivariate thinking: Basic themes and methods. New Jersey: Lawrence Erlbaum \& Associates.

Hartman CG, Straus WL. 1939. Relaxation of the pelvic ligaments in pregnant monkeys. American Journal of Obstetric Gynecology 37:498-500.

Harvey PH, Pagel MD. 1991. The Comparative Method in Evolutionary Biology. Oxford: Oxford University Press.

Hauser G, Jahn R. 1984. Sexual dimorphism in pelvic height. Journal of Human Evolution 13:589-592.

Häusler M, Schmid P. 1995. Comparison of the pelves of Sts 14 and AL288-1: implications for birth and sexual dimorphism in australopithecines. Journal of Human Evolution 29:363-383.

Hedrick AV, Temeles EJ. 1989. Thee evolution of sexual dimorphism in animals: Hypotheses and tests. Trends in Ecology \& volution 4:136-138.

Heyns OS. 1947. A preliminary report on the pelvic brim index. British Journal of Obstetrics and Gynecology 54:39-64. 
Holland EL, Cran GW, Elwood JH, Pinkerton JH, Thompson W. 1982. Associations between pelvic anatomy, height, and year of birth of men and women in Belfast. Annals Human Biology 9:113-120.

Hopf S. 1967. Notes on pregnancy, delivery, and infant survival in captive squirrel monkeys. Primates 8:323-332.

Houle D. 1992. Comparing evolvability and variability of quantitative traits. Genetics Society of America 130:195-204.

Howell FC. 1978. Hominidae. In: Evolution of African Mammals. Eds: MaglioVJ, Cooke HBS. Cambridge: Harvary University Press.

Howells WW, Hotelling H. 1936. Measurements and correlations on pelves of Indians of the Southwest. American Journal of Physical Anthropology 21:91-106.

Jagani N, Schulman H, Chandra P, Gonzalez R, Fleischer A. 1981. The predictability of labor outcome from a comparison of birth weight and x-ray pelvimetry. American Journal of Obstetric Gynecology 139:507-511.

Jerison HW. 1973. Evolution of the Brain and Intelligence. New York: Academic Press.

Johanson DC, Edey M. 1981. Lucy: the beinnings of humankind. New York: Simon ad Schuster.

Johanson DC, Lovejoy CO, Kimbel WH, White TD, Ward SC, Bush ME, Latimer BM, Coppens Y. 1982. Morphology of the Pliocene partial hominid skeleton (A.L. 
288-1) from the Hadar Formation, Ethiopia. American Journal of Physical Anthropology 57:403-451.

Johnston FE. 1961. Sequence of epiphyseal union in a prehistoric population from Indian Knoll. Human Biology 33:66-81.

Joulin D. 1864. Anatomie et physiologie comparee du bassin des Mammiferes. Archives Generales de Medecine:29-46.

Jungers WL. 1984. Aspects of size and scaling in primate biology with special reference to the locomotor skeleton. American Journal of Physical Anthropology 27:7397.

Jungers WL. 1988. Relative joint size and hominoid locomotor adaptations with implications for the evolution of hominid bipedalism. Journal of Human Evolution 17:247-265.

Kanthaswamy S, Jilian N, Hernandez-Pacheco R. Ruiz-Lambides A, Maldonado E, Martinez M, Sariol CA. 2016. The population genetic composition of conventioal and SPF clonies of rhesus macaques (Macaca mulatta) at the Caribbean Primate Research Center. Journal of the American Association for Laboratory Animal Science 55:147-155.

Kawecki TJ. 2008. Adaptation to marginal habitats. Annual Review of Ecology, Evolution, and Systematics 39:321-342. 
Kibii JM, Churchill SE, Schmid P, Carlson KJ, Reed ND, de Ruiter DJ, Berger LR. 2011. A partial pelvis of Australopithecus sediba. Science 333 1407-1411

Kimura K. 1982. A base-wing index for sexing the sacrum. Journaol of the Anthropological Society of Nippon 90:153-162.

Klingenberg CP. 2008. Morphological integration and developmental modularity. Annual Review of Ecology, Evolution, and Systematics 39:115-132.

Klingenberg CP. 2009. MorphoJ: an integrated software package for geometric morphometrics. Molecular Ecology Resources 11:353-357.

Klingenberg CP. 2011. MORPHOJ: an integrated software package for geometric morphometrics. Molecular Ecology Resources 11:353-357.

Klingenberg CP, Marugán-Lobón J. 2013. Evolutionary covariation in geometric morphometric data: analyzing integration, modularity, and allometry in a phylogenetic context. Systematic Biology 62:591-610.

Kohn L, Cheverud J, Bhatia G, Commean P, Smith K, Vannier MW. 1995. Anthropometric opical surface imagning system repeatability, precision, and validation. Annals of Plastic Surgery 34:362-371.

Krogman WM. 1951. The scars of human evolution. Scientific American 185:54-57.

Krogman WM, Işcan MY. 1986. The Human Skeleton in Forensic Medicine. Springfield, IL: Charles C. Thomas Publisher.

Krukierek. 1951. The sexual differences of the human pelvis. Gynecologia 132:92-110. 
Kurki HK. 2007. Protection of obstetric dimensions in a small-bodied human sample. American Journal of Physical Anthropology 133:1152-1165.

Kurki HK. 2011. Pelvic dimorphism in relation to body size and body size dimorphism in umans. Journal of Human Evolution 61:631-643.

Lachenbruch PA, Mickey MR. 1968. Estimation of error rates in discriminant analysis. Technometrics 10:1-11.

Lande R. 1979. Quantitative genetic analysis of multivariate evolution, applied to brain: body size allometry. Evolution 33:402-416.

Lande R. 1980a. The genetic covariance between characters maintained by pleiotropic mutations. Genetics 94:203-215.

Lande R. 1980b. Sexual dimorphism, sexual selection, and adaptation in polygenic characters. Evolution 34:292-305.

Lande R, Arnold SJ. 1983. The measurement of selection on correlated characters. Evolution 37:1210-1226.

LaVelle M. 1995. Natural selection and developmental sexual variation in the human pelvis. American Journal of Physical Anthropology 98:59-72.

Lele S, Richtsmeier JT. 1991. Euclidean distance matrix analysis: A coordinate-free approach for comparing biological shapes using landmark data. American Journal of Physical Anthropology 86:415-427. 
Lele S, Richtsmeier JT. 1995. Euclidean distance matrix analysis: Confidence intervals for form and growth differences. American Journal of Physical Anthropology 98:73-86.

Lele S, Richtsmeier JT. 2001. An invariant approach to the statistical analysis of shapes. London: Chapman and Hall-CRC Press.

Letterman GS. 1941. The greater sciatic notch in American whites and Negroes. American Journal of Physical Anthropology 28:99-116.

Leutenegger W. 1970. Beziehungen zwischen der neugeborenengrösse und dem sexualdimorphismus am becken bei simischen primaten. Folia Primatologica $12: 224-235$.

Leutenegger W. 1973. Sexual dimorphism in the pelves of African lorises. American Journal of Physical Anthropology 38:251-254.

Leutenegger W. 1974. Funcitonal aspects of pelvic morphology in simian primates. Journal of Human Evolution 3:207-222.

Leutenegger W. 1977. Functional interpretation of the sacrum of Australopithecus africanus. S African Journal of Science 73:308-310.

Leutenegger W. 1982. Encephalization and obstetrics in primates with particular reference to human evolution. In: Armstrong E, Falk D, editors. Primate Brain Evolution. New York: Plenum Press. 
Leutenegger W, Kelly JT. 1977. Relationship of sexual dimorphism in canine size and body size to social, behavioral, and ecological correlates in anthropoid primates. Primates 18:117-136.

Lewton KL. 2010. Locomotor Function and the Evolution of the Primate Pelvis. In: Anthropology: Arizona State University.

Lewton KL. 2012. Evolvability of the primate pelvic girdle. Evolutionary Biology 39: 126-139.

Lewton KL. 2015. Allometric scaling and locomotor function in the primate pelvis. American Journal of Physical Anthropology 156:511-530.

Li Y. 2002. Postnatal development of pelvic sexual dimorphism in four anthropoid primates. In. Baltimore, Maryland: Johns Hopkins University.

Lindburg DG. 1982. Primate obstetrics: the biology of birth. American Journal of Primatology 3:193-199.

Lockwood CA, Richmond BG, Jugers WL, Kimbel WH. 1996. Randomization procedures and sexual dimorphism in Australopithecus afarensis. Journal of Human Evolution 31:537-548.

Lovejoy CO. 1981. The origin of man. Science (New York, NY) 211:341-350.

Lovejoy CO. 2005. The natural history of human gait and posture Part 1. Spine and pelvis. Gait and Posture 21:95-112. 
Lovejoy CO, Cohn MJ, White TD. 1999. Morphological analysis of mammalian limbs: a developmental perspective. Proceedings of the National Academy of Sciences USA 96:13247-13252.

Lovejoy CO, Suwa G, Simpson SW, Matternes JH, White TD. 2009a. The great divides: Ardipithecus ramidus reveals the postcrania of our last common ancestors with African apes. Science 326:100-106.

Lovejoy CO, Suwa G, Spurlock L, Asfaw B, White TD. 2009b. The pelvis and femur of Ardipithecus ramidus: The emergence of upright walking. Science 326:71, 71e71-71e76.

Lovejoy CO, McCollum MA. 2010. Spinopelvic pathways to bipedality: why no hominids ever relied on a bent-hip bent-knee gait. Philosophical Transactions of the Royal Society B 365: 3289-3299.

Lycett SJ, von Cramon-Taubadel N. 2013. Understanding the comparative catarrhine context of human pelvic form: A 3D geometric morphometric analysis. Journal of Human Evolution 64:300-310.

MacLatchy LM. 1998. Reconstruction of hip joint function in extant and extinct fossil primates. In: Strasser E, Fleagle JG, Rosenberger AL, McHenry HM, editors. Primate locomotion: recent advances New York: Plenum Press.

Maclennan AH. 1991. The role of the hormone relaxin in human reproduction and pelvic girdle relaxation. Scandinavian Journal of Rheumatology Supplement $88: 7-15$. 
Magwene PM. 2001. New tools for studying integration and modularity. Evolution $55: 1734-1745$.

Malas MA, Sulak O. 2000. Measurements of anterior fontanelle during the fetal period. Obstetric Gynecology 20:601-605.

Manly BFJ. 1997. Randomization, bootstrap, and Monte Carlo methods in biology. Boca Raton, FL: CRC Press.

Marroig G, Cheverud J. 2001. A comparison of phenotypic variation and covariation patterns and the role of phylogeny, ecology, and ontogeny during cranial evolution of New World monkeys. Evolution 55:2576-2600.

Marroig G, Shirai LT, Porto A, de Oliveira FB, De Conto V. 2009. The evolutionary modularity in the mammalian skull II: evolutionary consequences. Evolutionary Biology 36:136-148.

Martin RD. 1983. Human Brain Evolution in an Ecological Context. New York: American Museum of Natural History.

McHenry HM. 1975. Biomechanical interpretation of the early hominid hip. Journal of Human Evolution 4:343-355.

McHenry HM. 1991. Sexual dimorphism in Australopithecus afarensis. Journal of Human Evolution 20:21-32.

McHenry HM. 1992. Body size and proportions in early hominids. American Journal of Physical Anthropology 87:407-431. 
Miller GS Jr. 1933. The groups and names of macaques In: The anatomy of the Rhesus monkey (Macaca mulatta). Eds: Hartman CG, Straus WL. Baltimore: Williams and Wilkins.

Milne N. 1990. Sexing of human hip bones. Journal of Anatomy 172:221-226.

Moffett EA, Aldridge K. 2013. Size of the anterior fontanelle: Three-dimensional measurement of a key trait in human evolution. Anatomical Record 297:234239.

Moffett EA, Maddux SD, Ward CV. 2013. Sexual dimorphism in relative sacral breadth among catarrhine primates. American Journal of Physical Anthropology 152:435-446.

Morbeck ME. 1976. Leaping, bounding, and bipeadlism in Colobus guereza: a spectrum of positional behavior. Yearbook of Physical Anthropology 20:408-420.

Morgan MA, Thurnau GR, Fishburne Jr. JI. 1986. The fetal-pelvic index as an indicator of fetal-pelvic disproportion: a preliminary report. American Journal of Obstetric Gynecology 155:608-613.

Morgan ME, Lewton KL, Kelley J, Otárola-Castillo E, Barry JC, Flynn LJ. 2015. A partial hominoid innominate from the Miocene of Pakistan: description and preliminary analysis. Proceedings of the National Academy of Sciences of the United States of America 112:82-87. 
Nakatsukasa M, Hirose Y. 2003. Scaling of lumbar vertebrae in anthropoids and implications for evolution of the hominoid axial skeleton. Primates 44:127-135.

Nicholson C, Allen HS. 1945. Variation in the female pelvis. The Lancet 248:192-195.

Ninness C, Newton R, Saxton J, Rumph R, Bradfield A, Harrison C, Vasquez E. 2002. Small group statistics: a Monte Carlo comparison of parametric and randomization tests. Behavior and Social Issues 12:53-63.

Nocon JJ, McKenzie DK, Thomas LJ, Hansell RS. 1993. Shoulder dystocia: An analysis of risks and obstetric maneuvers. American Journal of Obstetric Gynecology 168:1732-1739.

Novak L. 2007. Sexual dimorphism of the posterior pelvis of the Robert J. Terry Anatomical Collection and William M. Bass Donated Skeletal Collection. In: Department of Anthropology. Orlando: University of Central Florida.

Novak L, Schultz J, McIntyre M. 2012. Determining the sex of the posterior ilium from the Robert J. Terry and William M. Bass collections. Journal of Forensic Science 57:1155-1160.

Olson EC, Miller ER. 1951. A mathematical model applied to the evolution of species. Evolution 5:325-338.

Olson EC, Miller RL. 1958. Morphological Integration. Chicago: University of Chicago Press. 
Oxorn H. 1986. Oxorn-Foote Human Labor and Birth, 5 ed. Norwalk, Connecticut: Appleton-Century-Crofts.

Paladini D, Vassallo M, Sglavo G, Pastore G, Lapadula C, Nappi C. 2008. Normal and abnormal development of the fetal anterior fontanelle: a three-dimensional ultrasound study. Ultrasound in Obstetrics and Gynecology 32:755-761.

Paterson AM. 1893. The human sacrum. Scientific Transactions of the Royal Dublin Society 5:123-204.

Patriquin ML, Steyn M, Loth SR. 2005. Metric analysis of sex differences in South African black and white pelves. Forensic Science International 147:119-127.

Perini TA, de Oliveira GL, dos Santos Ornellas J, de Oliveira FP. 2005. Technical error of measurement in anthropometry. Revista Brasileira de Medicina do Esporte 11:86-90.

Phillips IR. 1976. The reproductive potential of the common cotton-eared marmoset (Callithrix jacchus) in captivity. Journal of Medical Primatology 5:49-55.

Pinkerton JH. 1973. Some aspects of the evolution and comparative anatomy of the human pelvis. Journal of Obstetrics and Gynaecology of the British Commonwealth 80:97-102.

Plavcan JM. 2001. Sexual dimorphism in primate evolution. American Journal of Physical Anthropology 44:25-53. 
Plavcan JM. 2011. Understanding dimorphism as a function of changes in male and female traits. Evolutionay Anthropology 20:143-155.

Plavcan JM. 2012. Sexual size dimorphism, canine dimorphism, and male-male competition in primates: Where do humans fit in? Human Nature 23:45-67.

Pocock, 1932. The rhesus macaques (Macaca mulatta). Journal of Bombay Natural History Society 35: 530-551.

Ponce de León MS, Golovanova L, Doronichev V, Romanova G, Akazawa T, Kondo O, Ishida H, Zollikofer CPE. 2008. Neanderthal brain size at birth provides insights into the evolution of human life history. Proceedings of the National Academy of Science USA 105:13764-13768.

Popich GA, Smith DW. 1972. Fontanels: range of normal size. Journal of Pediatrics 80:749-752.

Porto A, de Oliveira FB, Shirai LT, De Conto V, Marroig G. 2009. The evolution of modularity in the mammalian skull I: Morphological integration patterns and magnitudes. Evolutionary Biology 36:118-135.

Rak Y, Arensburg B. 1987. Kebara 2 Neanderthal pelvis: first look at a complete inlet. American Journal of Physical Anthropology 73:227-231.

Rasmussen KM, Ausman LM, Hayes KC. 1980. Vital statistics from a laboratory breeding colony of squirrel monkeys (Saimiri sciureus). Laboratory Animal Science 30:99-106. 
Ravosa MJ, Ross CF. 1994. Craniodental allometry and heterochrony in two howler monkeys: Alouatta seniculus and A. palliata. American Journal of Primatology $33: 277-299$.

Remis M. 1995. Effects of body size and social context on the arboreal activities of lowland gorillas in the Central African Republic. American Journal of Physical Anthropology 97:413-433.

Reno PL. Meindl RS, McCollum MA, Lovejoy CO. 2003. Sexual dimorphism in Australopithecus afarensis was similar to that of modern humans. Proceedings of the National Academy of Sciences 100:9404-9409.

Rensch B. 1960. Evolution Above the Species Level. New York: Columbia University Press.

Reynolds E. 1931. The evolution of the human pelvis in relation to the mechanics of erect posture. Papers of the Peabody Museum of American Archaeology and Ethnology, Harvard University Cambridge.

Reynolds E, Hooton EA. 1936. Relation of the pelvis to erect posture: an exploratory study. American Journal of Physical Anthropology 21:253-278.

Richmond BG, Jugers WL. 1995. Size variation and sexual dimorphism in Australopithecus afarensis and living hominoids. Journal of Human Evolution 29:229-245. 
Ridley M. 1995. Pelvic sexual dimorphism and relative neonatal brain size really are related. American Journal of Physical Anthropology 97:197-200.

Ripley B. 2016. Package 'boot': Bootstrap functions. In: R Package version 2.3.2

Robinson JT. 1972. Early Hominid Posture and Locomotion. Chicago: University of Chicago Press.

Roff DA. 2006. Introduction to Computer-intensive Methods of Data Analysis in Biology: Cambridge University Press.

Rohlf FJ, Corti M. 2000. The use of two-block partial least-squares to study covariation in shape. Systematic Zoology 49:740-753.

Rose MD. 1985. A hominine hip bone, KNM-ER 3228, from East Lake Turkana, Kenya. American Journal of Physical Anthropology 63:371-378.

Rosenberg K, Zuné L, Ruff CB. 2006. Body size, body proportions, and encephalization in a Middle Pleistocene archaic human from northern China. Proceedings of the National Academy of Science USA 103:3552-3556.

Rosenberg KR. 1992. The evolution of modern human childbirth. American Journal of Physical Anthropology 35:89-124.

Rosenberg KR, Trevathan WR. 1996. Bipedalism and human birth: the obstetrical dilemma revisited. Evolutionary Anthropology 4:161-168.

Rosenberg KR, Trevathan WR. 2002. Birth, obstetrics, and human evolution. British Journal of Obstetrics and Gynecology 109:1199-1206. 
Ruff CB. 1987. Sexual dimorphism in human lower limb bone structure: relationship to subsistence strategy and seuxal division of labor. Journal of Human Evolution 6:391-416.

Ruff CB. 1995. Biomechanics of the hip and birth in early Homo. American Journal of Anatomy 98:527-574.

Ruff CB, Hayes WC. 1983. Cross-sectional geometry of Pecos Pueblo femora and tibiae - A biomechanical investigation: II. Sex, age, and side differences. American Journal of Physical Anthropology 60:383-400.

Schultz AH. 1930. The skeleton of the trunk and limbs of higher primates. Human Biology 2:302-438.

Schultz AH. 1936. Characters common to higher primates and characters specific for man. Quarterly Review Biology 11:425-455.

Schultz AH. 1944. Age changes and variability in gibbons. A morphological study on a population sample of man-like apes. American Journal of Physical Anthropology 2:1-129.

Schultz AH. 1949. Sex differences in the pelves of primates. American Journal of Physical Anthropology 7:401-423.

Schultz AH. 1961. Vertebral column and thorax. New York: Karger.

Schultz AH. 1969. The Life of Primates. New York: Universe Books. 
Schultz AH, Straus WL. 1945. The numbers of vertebrae in primates. Proceedings of the American Philosophical Society 89:601-626.

Schutz H, Donovan ER, Hayes JP. 2009a. Effects of parity on pelvic size and shape dimorphism in Mus. Journal of Morphology 270:834-842.

Schutz H, Polly PD, Krieger JD, Guralnick RP. 2009b. Differential sexual dimorphism: size and shape in the cranium and pelvis of grey foxes (Urocyon) Biological Journal of the Linnean Society 96:339-353.

Scott JE. 2010. Nonsocial influences on canine size in anthropoid primates (Ph.D.). Tempe: Arizona State University.

Segebarth-Orban R. 1980. An evaluation of the sexual dimorphism of the human innominate bone. Journal of Human Evolution 9:601-607.

Shajari H, Rashidiranjbar N, Ashrafi M. 2011. Anterior fontanelle size in healthy Iranian neonates on the first day of life. Acta Medica Iran 49:543-546.

Sherwood OD. 1988. Relaxin. In: Knobil E, Neill J, editors. The Physiology of Reproduction. New York: Raven Press.

Shine R. 1989. Ecological causes for the evolution of sexual dimorphism: a review of the evidence. Quarterly Review of Biology 64:419-461.

Simpson SW, Quade J, Levin NE, Butler R, Dupont-Nivet G, Everett M, Semaw S. 2008. A female Homo erectus pelvis from Gona, Ethiopia. Science 322:10891092. 
Smith RJ. 2009. Use and misuse of the reduced major axis for line-fitting. American Journal of Physical Anthropology 140:476-486.

Smith RJ, Jungers WL. 1997. Body mass in comparative primatology. Journal of Human Evolution 32:523-559.

Steele KB, Javert CT. 1942. The mechanism of labor for transverse positions of the vertex. Surg Gynecol Obstet 75:477-484.

Stern JT, Sussman RL. 1983. The locomotor anatomy of Australopithecus afarensis. American Journal of Physical Anthropology 60:279-317.

Steudel K. 1981a. Body size estimators in primate skeletal material. International Journal of Primatology 2:81-90.

Steudel K. 1981b. Functional aspects of primate pelvic structure: a multivariate approach. American Journal of Physical Anthropology 55:399-410.

Steudel K. 1981c. Sexual dimorphism and allometry in primate os coxae. American Journal of Physical Anthropology 55:209-215.

Stoller MK. 1995. The obstetric pelvis and mechanism of labor in nonhuman primates. In: Department of Anthropology. Chicago, Illinois: University of Chicago.

Storey JD. 2015. Q-value estimation for false discovery rate control. In: R Package version 2.3.2.

Tague RG. 1989. Variation in pelvic size between males and females. American Journal of Physical Anthropology 80:59-71. 
Tague RG. 1991. Commonalities in dimorphism and variability in the anthropoid pelvis, with implications for the fossil record. Journal of Human Evolution 21:153-176.

Tague RG. 1992. Sexual dimorphism in the human bony pelvis, with a consideration of the Neandertal pelvis from Kebara Cave, Israel. American Journal of Physical Anthropology 88:1-21.

Tague RG. 1993. Pubic sympheseal synostosis and sexual dimorphism of the pelvis is Presbytis cristata and Presbytis rubicunda. International Journal of Primatology $14: 637-654$.

Tague RG. 1995. Variation in pelvic size between males and females in nonhuman anthropoids. American Journal of Physical Anthropology 97:213-233.

Tague RG. 2000. Do big females have big pelves? American Journal of Physical Anthropology 112:377-393.

Tague RG. 2003. Pelvic sexual dimorphism in a metatherian, Didelphis virginiana: implications for eutherians. J Mammal 84:1464-1473.

Tague RG. 2007. Costal process of the first sacral vertebra: sexual dimorphism and obstetrical adaptation. American Journal of Physical Anthropology 132:395405.

Tague RG. 2012. Small anatomical variant has profound implications for evolution of human birth and brain development. Proceedings of the National Academy of Science USA 109:8360-8361. 
Tague RG, Lovejoy CO. 1986. The obstetric pelvis of A.L. 288-1 (Lucy). Journal of Human Evolution 15:237-255.

Tague RG, Lovejoy CO. 1998. AL 288-1-- Lucy or Lucifer: gender confusion in the Pliocene. Journal of Human Evolution 35:75-94.

Thoms H. 1937. Pelvic variations in 300 primiparous white women. Surg Gynecol Obstet 64 .

Thoms H, Greulich WW. 1940. A comparative study of male and female pelves. American Journal of Obstetric Gynecology 39:56-62.

Thorp DJ, Fray WE. 1938. The pelvic joints during pregnancy and labor. Journal of the American Medical Association 111:1162-1166.

Toussaint M, Macho GA, Tobias PV, Partridge TC, Hughes AR. 2003. The third partial skeleton of a late Pliocene hominin (Stw 431) from Sterkfontein, South Africa. S Afr J Sci 99:215-223.

Trevathan W. 1987. Human Birth: An Evolutionary Perspective. Chicago: Aldine.

Trevathan WR. 1996. The evolution of bipedalism and assisted birth. Med Anthropol Q 10:287-298.

Trevathan WR, Rosenberg KR. 2000. The shoulders follow the head: postcranial constraints on human childbirth. Journal of Human Evolution 39:583-586.

Trotter M. 1926. The sacrum and sex. American Journal of Physical Anthropology 4:445-450. 
Turner WT. 1885. The index of the pelvic brim as a basis for classification. Journal of Anatomy and Physiology 20:125-143.

Villmoare B, Fish J, Jungers W. 2011. Selection, morphological integration, and strepsirrhine locomotor adaptations. Evolutionary Biology 38:88-99.

Wagner GP. 1996. Homologues, natural kinds and the evolution of modularity. American Zoologist 36:36-43.

Wagner GP, Altenberg L. 1996. Perspective: Complex adaptations and the evolution of evolvability. Evolution 50:967-976.

Wagner GP, Pavlicev M, Cheverud J. 2007. The road to modularity. Nature Reviews Genetics 8:921-931.

Walker A, Ruff CB. 1993. The reconstruction of the pelvis. In: Walker A, Leakey R, editors. The Nariokotome Homo erectus Skeleton. Cambridge: Harvard University Press.

Walrath D. 1997. Sexual dimorphism of the pelvis and its relationship to birth in human evolution. In. Philadelphia, PA: University of Pennsylvania.

Walrath D. 2003. Rethinking pelvic typologies and the human birth mechanism. Current Anthropology 44:5-31.

Walrath D, Glantz M. 1996. Sexual dimorphism in the pelvic midplane and its relationship to Neandertal reproductive patterns. American Journal of Physical Anthropology 100:89-100. 
Ward CV. 1991. Functional anatomy of the lower back and pelvis of the Miocene hominoid Proconsul nyanzae from Mfangano Island, Kenya In. Baltimore: The Johns Hopkins University.

Ward CV. 1993. Torso morphology and locomotion in Proconsul nyanzae. American Journal of Physical Anthropology 92: 291-398.

Ward CV, Feibel CS, Hammond AS, Leakey LN, Moffett EA, Plavcan JM, Skinner MM, Spoor F, Leakey MG. 2015. Journal of Human Evolution 81:48-67.

Warrener AG, Lewton KL, Pontzer H, Lieberman DE. 2015. A wider pelvis does not increase locomotor cost in humans, with implications for the evolution of childbirth. PloS one.

Warton DI, Wright IJ, Falster DS, Westoby M. 2006. Bivariate line-fitting methods for allometry. Biol Rev Camb Philos Soc 81:259-291.

Washburn SL. 1948. Sex differences in the pubic bone. American Journal of Physical Anthropology 6:199-207.

Washburn SL. 1950. The analysis of primate evolution with particular reference to the origin of man. Cold Spring Harbor Symposium on Quantitative Biology 15:6778.

Washburn SL. 1960. Tools and human evolution. Scientific American 203:63-75.

Waterman HC. 1929. Studies on the evolution of the pelvis of man and other primates. Bulletin of the American Museum of Natural History 58:585-642. 
Weaver TD. 2002. A Multi-Causal Functional Analysis of Hominid Hip Morphology. In. California: Stanford University.

Weaver TD, Hublin JJ. 2009. Neandertal birth canal shape and the evolution of human childbirth. Proceedings of the National Academy of Science USA 106:81518156.

Weiss G. 1984. Relaxin. Annual Review of Physiology 46:43-52.

Weiss G, Steinetz B, Dierschke D, Fritz G. 1981. Relaxin secretion in the rhesus monkey. Biology of Reproduction 24:565-567.

Wells JCK, DeSilva JM, Stock JT. 2012. The obstetric dilemma: an ancient game of Russian roulette, or a variable dilemma sensitive to ecology? Yearbook of Physical Anthropology 55:40-71.

Willmore KE, Roseman CC, Rogers J, Cheverud J, Richtsmeier JT. 2009. Comparison of mandibular phenotypic and genetic integration between baboon and mouse. Evolutionary Biology 36:19-36.

Willmore KE, Young NM, Richtsmeier JT. 2007. Phenotypic variability: its components, measurement, and underlying developmental processes. Evolutionary Biology 34:99-120.

Wittman AB, Wall LL. 2007. The evolutionary origins of obstructed labor: Bipedalism, encephalization, and the human obstetric dilemma. Obstetrical \& Gynecological Survey 62:739-748. 
Young M, Ince JG. 1940. A radiographic comparison of the male and female pelvis. Journal of Anatomy 74:374-385.

Young NM, Wagner GP, Hallgrimsson B. 2010. Development and the evolvability of human limabs. Proceedings of the National Academy of Science USA $107: 3400-3405$.

Zaretsky MV, Alexander JM, McIntire DD, Hatab MR, Twickler DM, Leveno KJ. 2005. Magnetic Resonance Imaging Pelvimetry and the Prediction of Labor Dystocia. Obstetric Gynecology 106:919-926.

Zelditch ML, Swiderski DL, Sheets HD, Fink WL. 2004. Geometric Morphometrics for Biologists: A Primer. San Diego, California: Elsevier Academic Press.

Zelditch ML, Wood AR, Swiderski DL. 2009. Building developmental integration into functional systems: Function induced integration of mandibular shape. Evolutionary Biology 36:71-87.

Zihlman A. 1978. Interpretations of early hominid locomotion. In: Jolly CJ, editor. Early Hominids in Africa. London: Duckworth. p 361-377. 
APPENDIX A
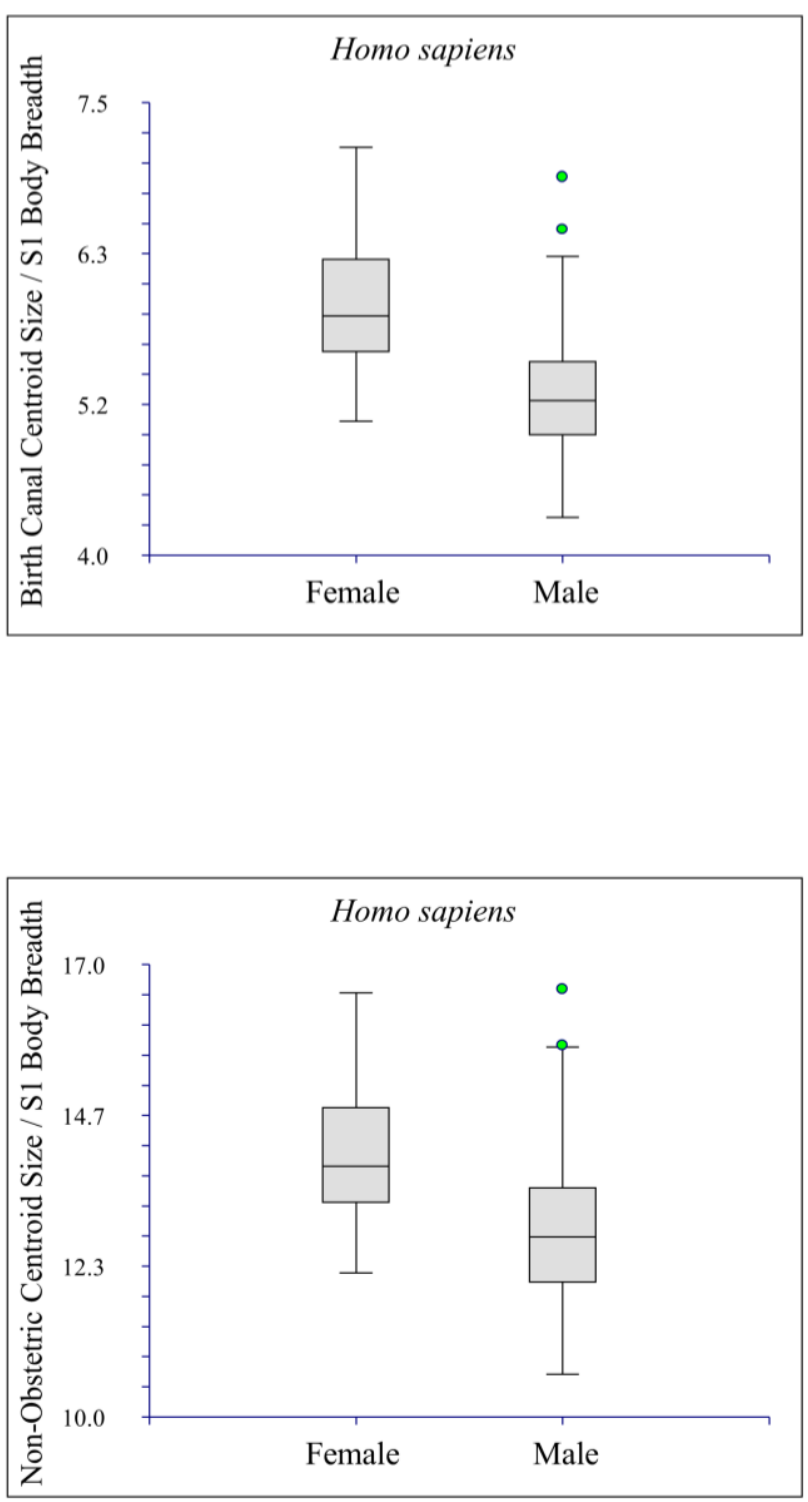

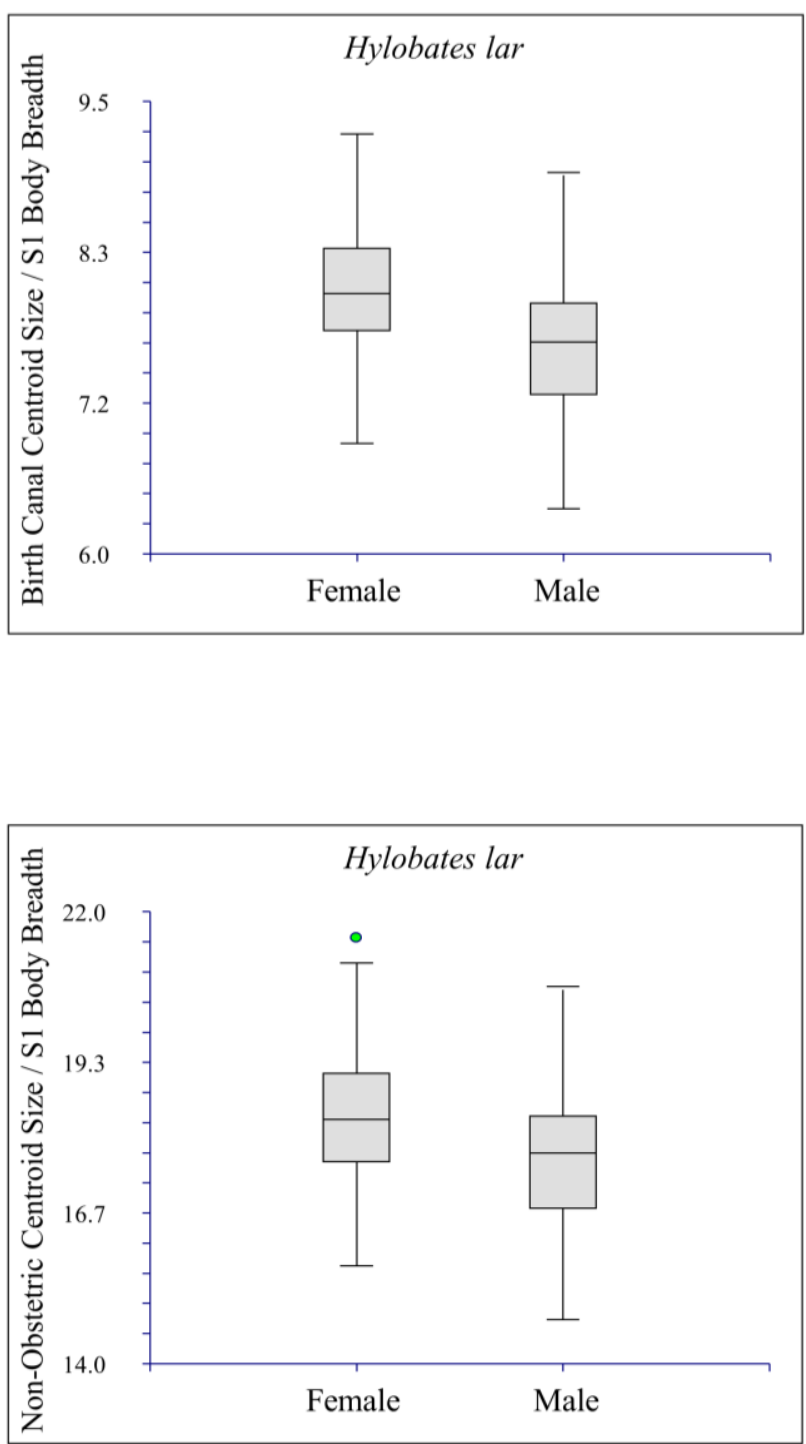

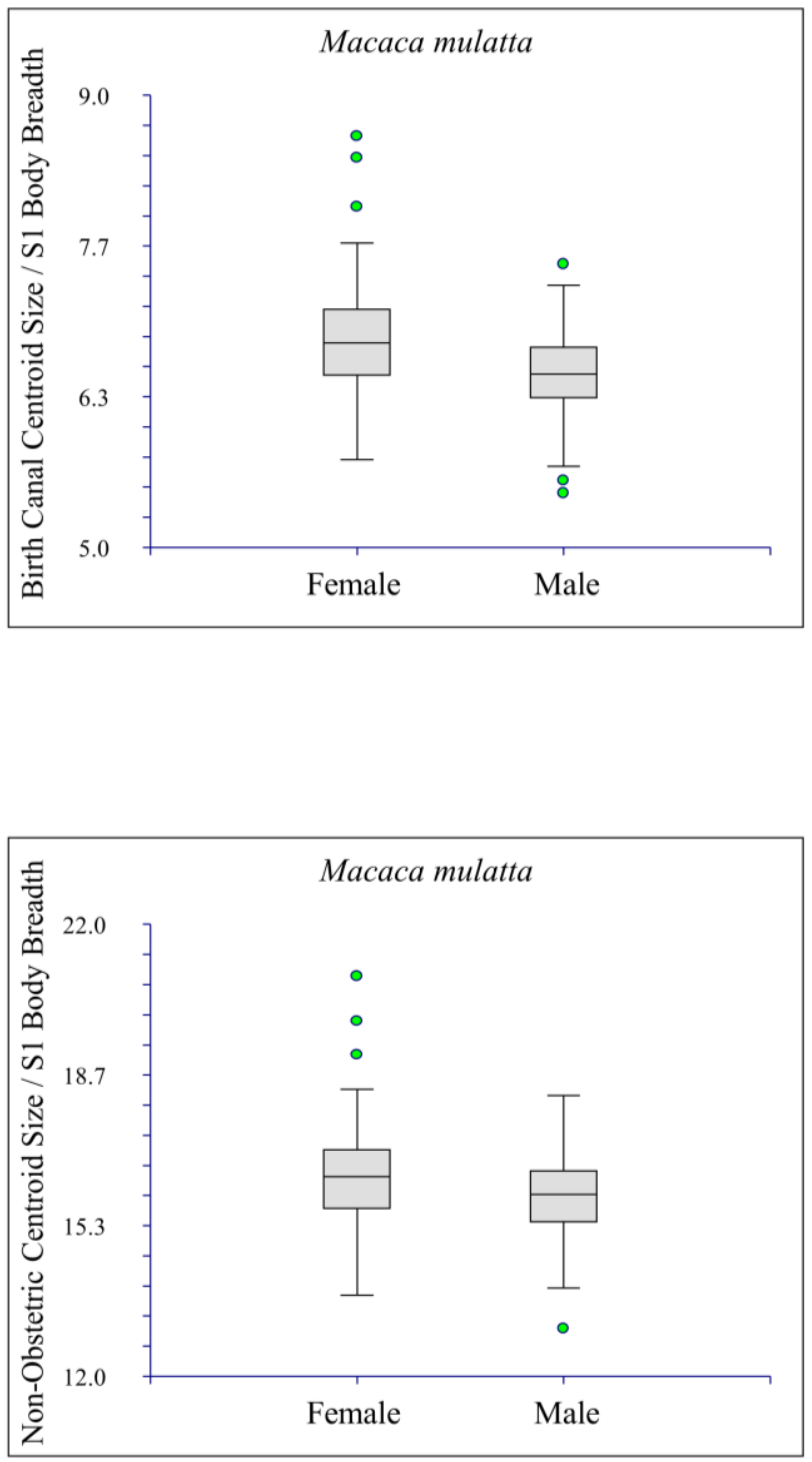

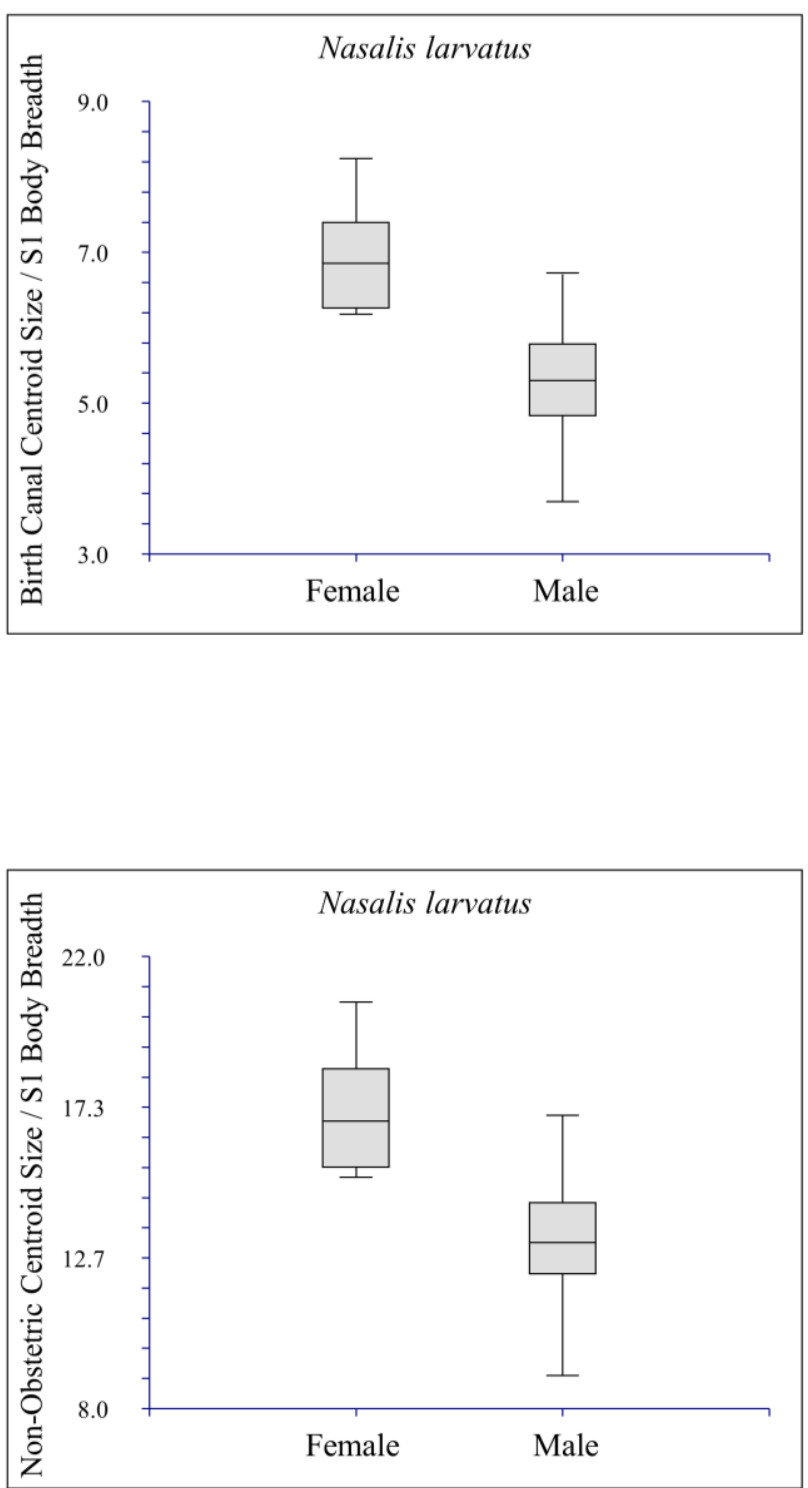

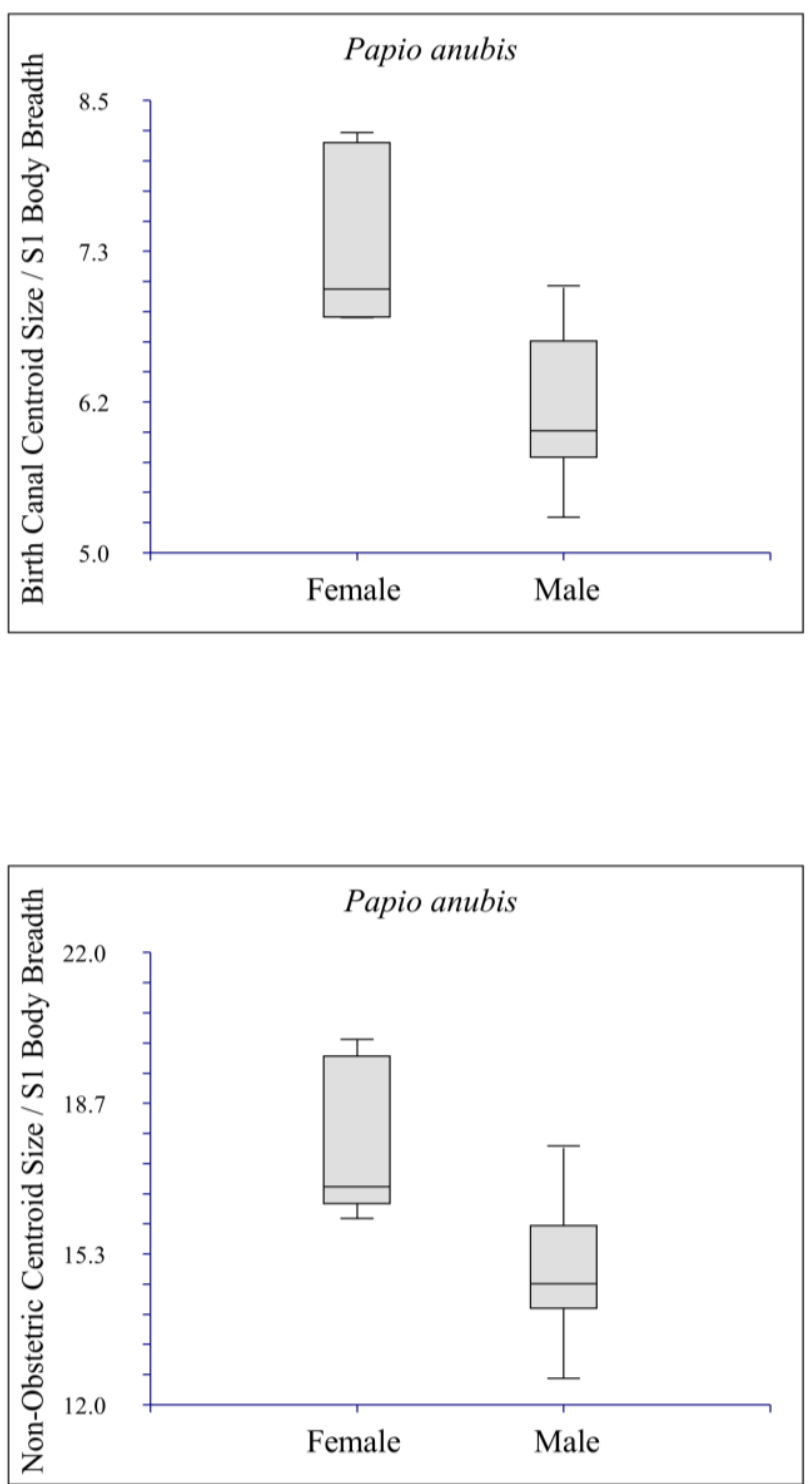

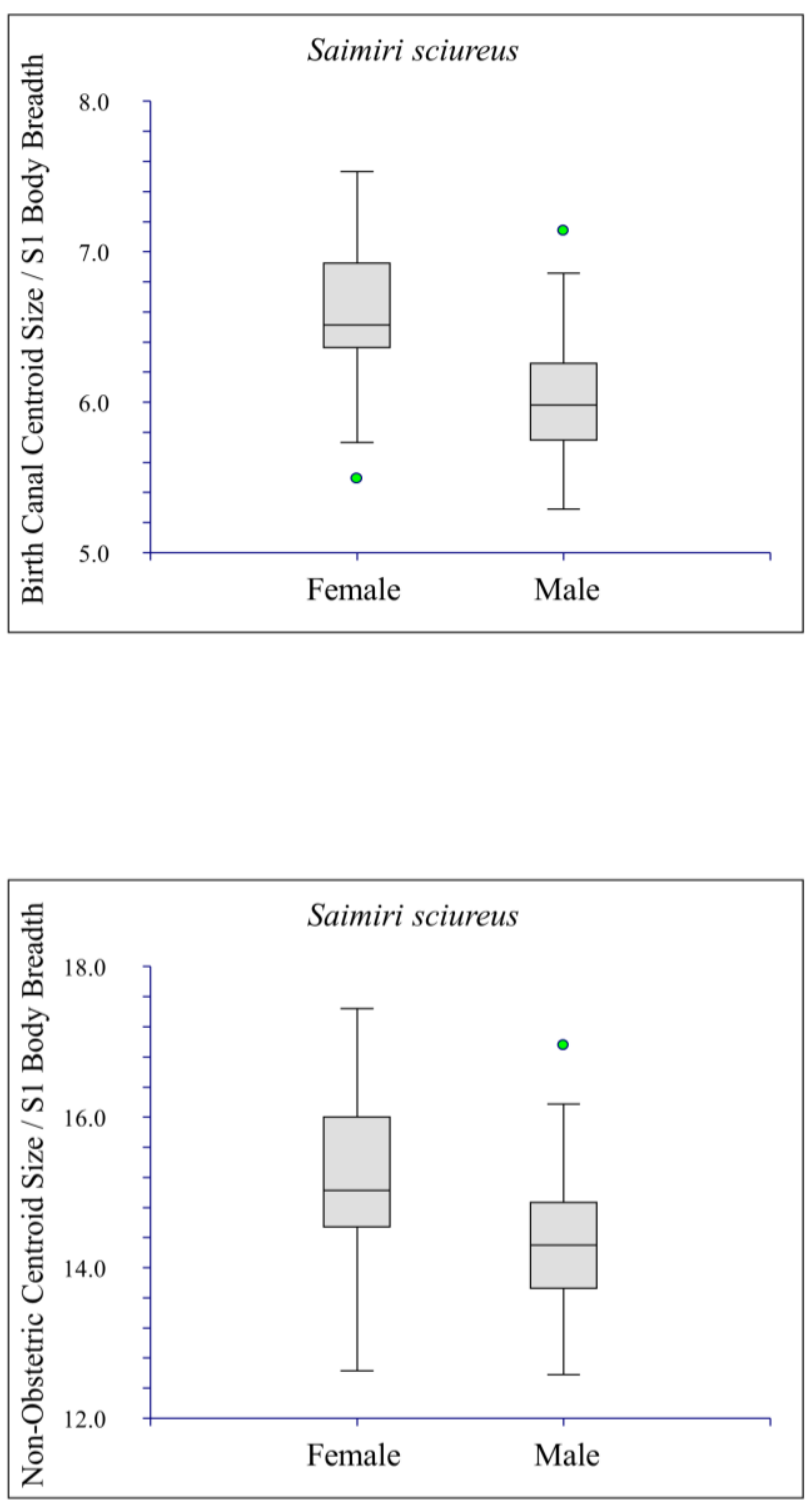

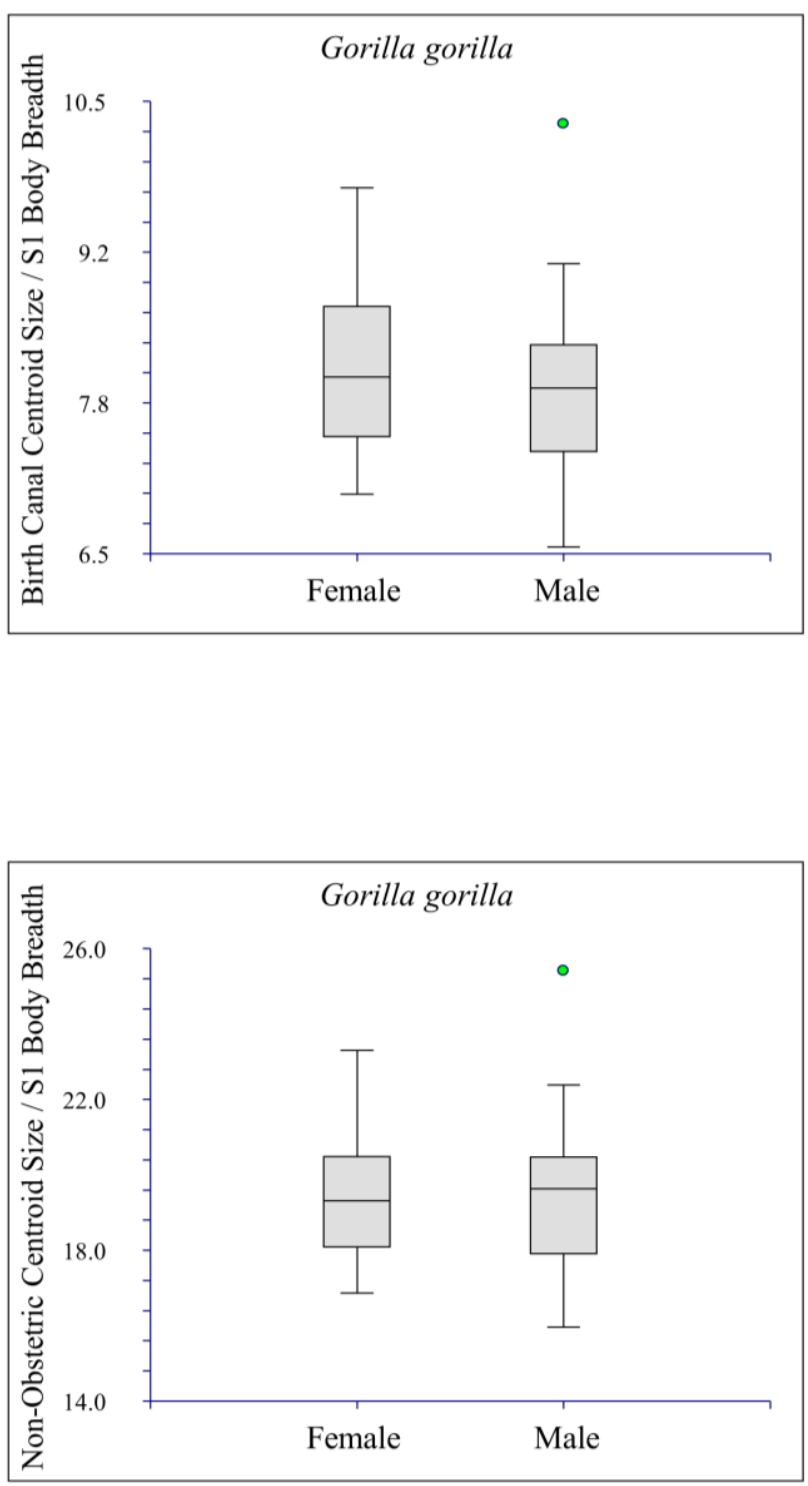

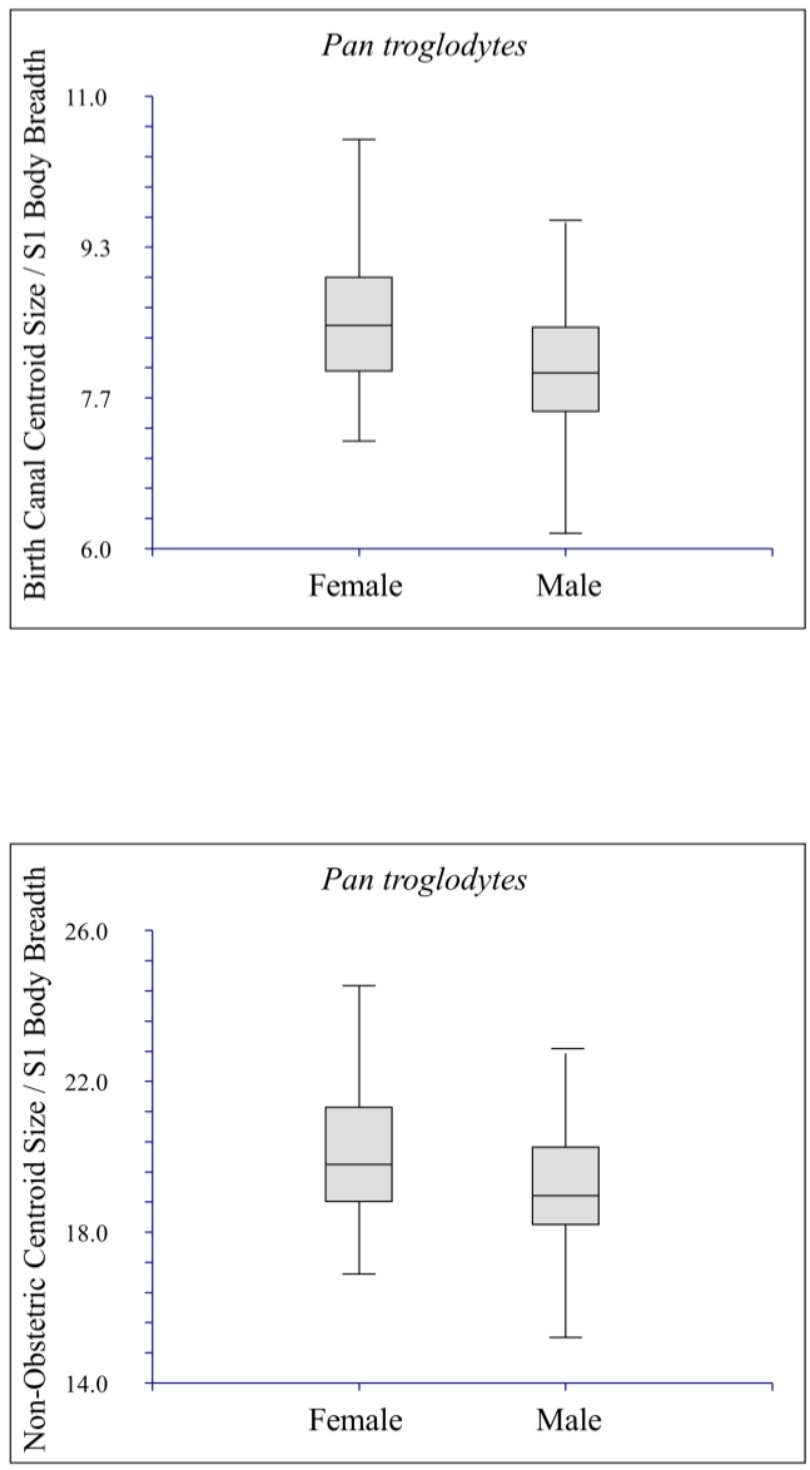

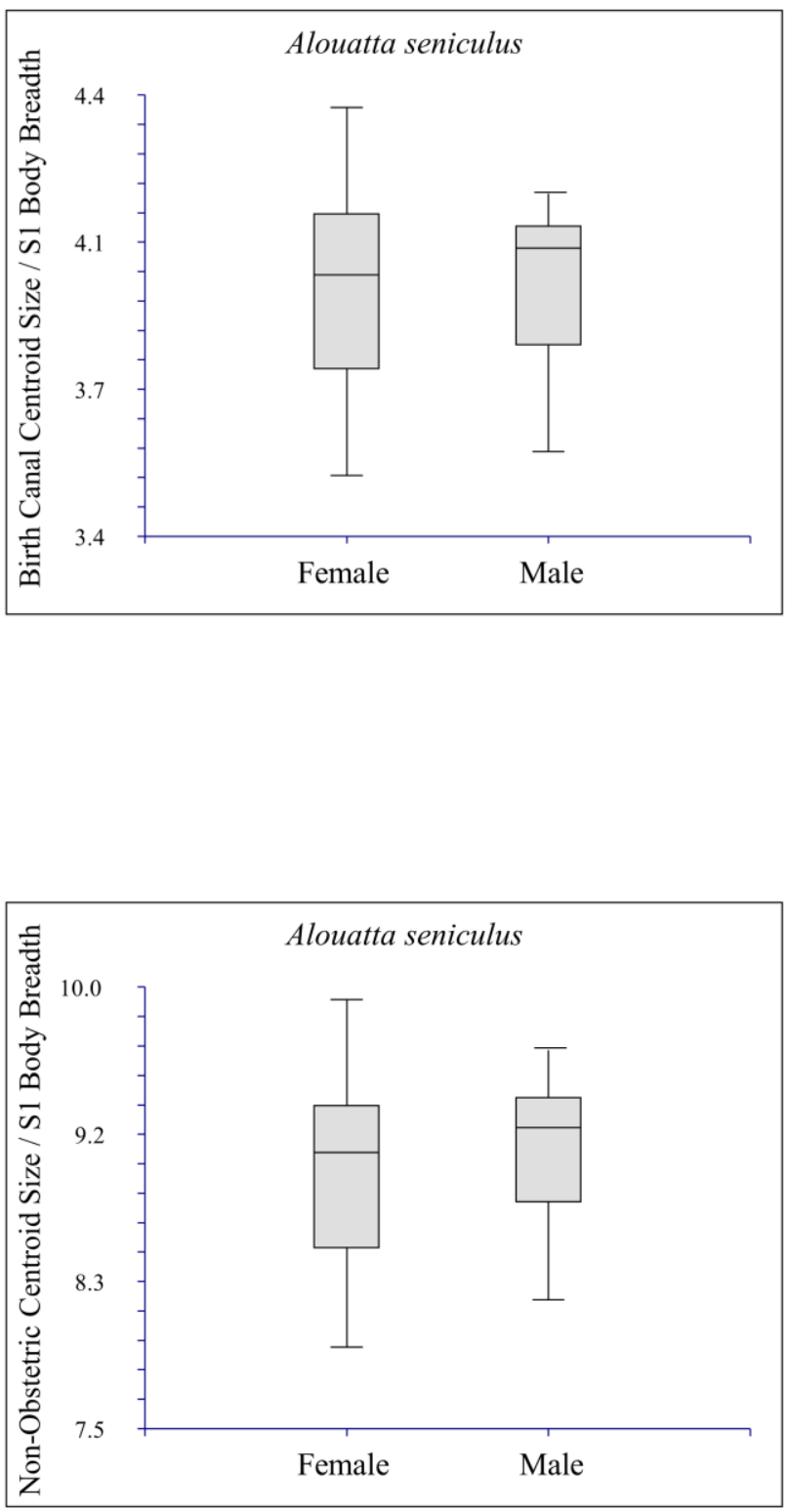


\section{VITA}

Elizabeth Moffett attended Louisiana State University for her Bachelors degree in Anthropology, and it was there that she discovered an interest in osteology and pelvic dimorphism. She attended the University of Missouri for her doctoral degree. During her time in the MU Department of Pathology and Anatomical Sciences, she participated in paleontological fieldwork in Kanapoi, Kenya, and traveled to Europe and Puerto Rico for data collection. She also discovered an interest in gross anatomy and functional morphology in graduate school. These experiences have shaped her early career. 